BURIED AND SURFACE MINE DETECTION FROM THERMAL IMAGE TIME SERIES

\author{
A THESIS SUBMITTED TO \\ GRADUATE SCHOOL OF NATURAL AND APPLIED SCIENCES \\ $\mathrm{OF}$ \\ MIDDLE EAST TECHNICAL UNIVERSITY
}

BY

SERKAN KAYA

IN PARTIAL FULFILLMENT OF THE REQUIREMENTS

FOR

THE DEGREE OF MASTER OF SCIENCE

IN

GEODETIC AND GEOGRAPHICAL INFORMATION TECHNOLOGIES

MAY 2016 

Approval of thesis:

\section{BURIED AND SURFACE MINE DETECTION FROM THERMAL IMAGE TIME SERIES}

submitted by SERKAN KAYA in partial fulfillment of the requirements for the degree of Master of Science in Geodetic and Geographical Information Technologies Department, Middle East Technical University by,

Prof. Dr. Gülbin Dural Ünver

Dean .,Graduate School of Natural and Applied Sciences

Assoc. Prof. Dr. Uğur Murat Leloğlu

Head of Dept.,

Geodetic and Geographical Information Technologies, METU

Assoc. Prof. Dr. Uğur Murat Leloğlu

Supervisor,

Geodetic and Geographical Information Technologies Dept., METU

Examining Committee Members:

Prof. Dr. Ahmet Coşar

Computer Engineering Dept., METU

Assoc. Prof. Dr. Uğur Murat Leloğlu

Head of Dept.,

Geodetic and Geographical Information Technologies, METU

Prof. Dr. Önder Haluk Tekbaş

Dean, Institute of Defense Sciences, Turkish Military Academy

Assoc. Prof. Dr. Hasan Koçer

Head of Dept., Electronics Engineering, Turkish Military Academy

Assoc. Prof. Dr. Ilkay Ulusoy Parnas

Electrical and Electronics Engineering Dept., METU

Date : $\quad 26 / 05 / 2016$ 
I hereby declare that all information in this document has been obtained and presented in accordance with academic rules and ethical conduct. I also declare that, as required by these rules and conduct, I have fully cited and referenced all material and results that are not original to this work.

Name, Last name : Serkan Kaya

Signature

iv 


\title{
ABSTRACT \\ BURIED AND SURFACE MINE DETECTION FROM THERMAL IMAGE TIME SERIES
}

\author{
KAYA, Serkan \\ M.Sc., Department of Geodetic and Geographic Information Technologies \\ Supervisor: Assoc. Prof. Dr. Uğur Murat LELOĞLU
}

May 2016, 117 pages

Since many countries suffer from existence of landmines in their territory, there is a growing demand for reliable landmine detection systems. Most of these systems require an operator to enter into the minefield. However, infrared sensor methods can be used remotely; hence, they do not put lives at risk during the search operation. In this thesis, a new approach to the infrared sensor method, which gives promising results, is presented.

Buried landmines generate specific spatiotemporal thermal image patterns on the surface. Noise-reduced thermal image time series were used after preprocessing with a circularly symmetric filter different from other studies. Supervised classification methods (Support Vector Machine, Mahalanobis Discriminant Analysis, Quadratic Discriminant Analysis, and K-Nearest Neighbor) are applied on the filtered image series.

Proposed method gives promising solutions that were verified with enlarged data sets. Different parameters (humidity, burial depths, training sample sizes, time intervals, seasons, spatial filter's sizes etc.) influences on the solutions were examined. To find the 
most useful time intervals, a 2-D heat transfer simulation was performed. Particularly using image series within nighttime, sunrise, and sunset times allow finding fourcentimeter deep plastic and 6.8-centimeter deep metal practice landmines with the proposed method. The best solutions were got with the 50-pixel outer diameter spatial filter and the 24-sample on this diameter. Detection rates were calculated higher in quasi-humid soil than dry soil.

Keywords: Thermal Infrared Imaging, Landmine Detection, Spatiotemporal Pattern Recognition, Supervised Classification, Thermal Simulation 


\title{
$\ddot{O} \mathrm{z}$
}

\section{ISIL KIZILÖTESİ GÖRÜNTÜ SERILLERİ İLE GÖMÜLÜ VE YÜZEY MAYINLARININ TESPITI}

\author{
KAYA, Serkan \\ Yüksek Lisans, Jeodezi ve Coğrafi Bilgi Sistemleri Bölümü \\ Tez Yöneticisi: Doç. Dr. Uğur Murat LELOĞLU
}

Mayıs 2016, 117 Sayfa

Birçok ülke topraklarındaki kara mayınlarının varlığından mağdur durumda olduğundan, güvenilir mayın tespit sistemlerine olan talep giderek artmaktadır. Bu sistemlerin çoğu operatörün mayın tarlasına girmesini gerektirmektedir. Oysaki kızıl ötesi sensör sistemleri uzaktan kullanılabilmektedir. Böylelikle kimsenin hayatı, arama operasyonu sırasında, bu yöntemler sayesinde riske atılmamaktadır. Bu tezde, umut verici sonuçlar veren, kızıl ötesi sensör yöntemine yeni bir yaklaşım sunulmaktadır.

Gömülü mayınlar toprak yüzeyinde farklı 1sıl görüntüler oluşturur. Bu farklar görüntü serilerinde görülebilir. Diğer çalışmalardan farklı olarak, gürültüsü azaltıldıktan sonra dairesel simetrik süzgeç ile ön işlemeye tabi tutulmuş görüntü serileri kullanılmıştır. Denetimli sınıflandırma yöntemleri (Destekçi Vektör Makinesi, Mahalanobis Diskriminant Analizi, İkinci Dereceden Diskriminant Analizi ve K-En Yakın Komşu) filtrelenmiş görüntü serilerine uygulanmıştır. 
Önerilen yöntem, genişletilmiş veri setleriyle kanıtlandığı gibi, umut verici sonuçlar vermiş̧ir. Farklı parametrelerin (toprağın nemi, gömülme derinlikleri, eğitim setti büyüklükleri, zaman aralıkları, mevsim, uzamsal süzgeçin ölçüleri vb.) sonuçlar üzerinde ki etkileri incelenmiştir. En uygun zaman aralıklarını tespit edebilmek için iki boyutlu 1sı transfer simülasyonu uygulanmıştır. Özellikle gece, gün doğumu ve gün batımı saatlerini kapsayan görüntü serileri, dört santimetre derinlikte plastik ve 6.8 santimetre derinlikte metal eğitim mayınlarının önerilen yöntemle bulunmasına imkan vermiştir. En iyi sonuçlar 50-piksel dış çaplı ve 24-örnekli uzamsal süzgeç ile alınmıştır. Tespit oranları nemli toprakta kuru toprağa oranla daha yüksek çıkmıştır.

Anahtar Kelimeler: Kızıl Ötesi Görüntüleme, Kara Mayın Tespiti, Zaman-Uzamsal Örüntü Tanıma, Denetimli Sınıflandırma, Isıl Simülasyon 
To My Beloved Family 


\section{ACKNOWLEDGEMENTS}

The author wishes to express his deepest gratitude to his supervisor Assoc. Prof. Dr. Uğur Murat LELOĞLU for his guidance, advice, criticism, encouragements, and insight throughout the research.

The author would like to thank Esen System Integration in order to their technical, and $\mathrm{ATOM}^{\mathrm{TM}} 1024$ camera support.

The author also wishes to thank Turkish Land Forces for supplying practice landmines and working areas and Turkish Military Academy to FLIR T 650 SC camera support.

The technical assistance of Mr. Ali Ömer KOZAL is gratefully acknowledged. 


\section{TABLE OF CONTENTS}

ABSTRACT

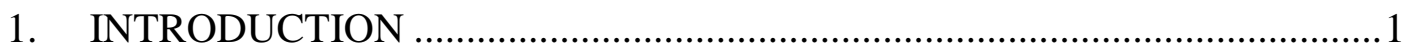

1.1 What are Landmines?................................................................ 1

1.2 Landmine Detection and Minefield Cleaning ..................................... 3

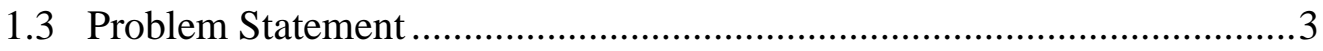

1.4 Advantages of the Proposed Method ....................................................

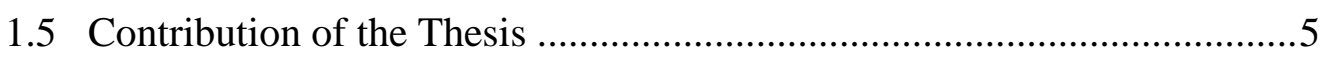

2. BACKGROUND INFORMATION AND LITERATURE REVIEW .............7

2.1 Heat Flow on the Soil Surface ........................................................ 7

2.2 Karhunen-Loève Transformation (KLT) .............................................. 8

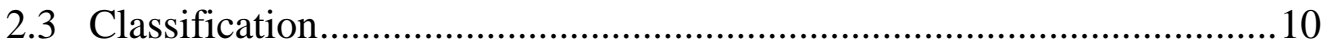

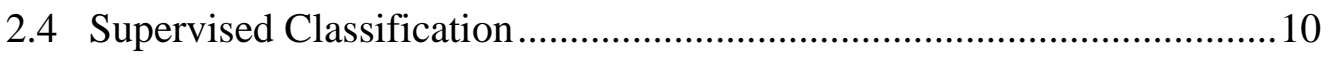

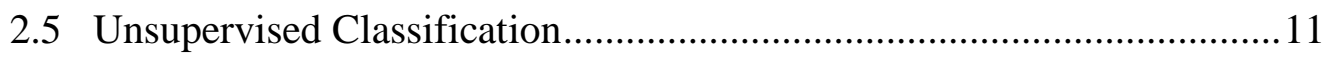

2.6 Landmine Detection Methods .................................................................. 11

2.7 Thermal Infrared in Landmine Detection .........................................13

3. PREPARATION OF PRACTICE LANDMINES AND THE TEST

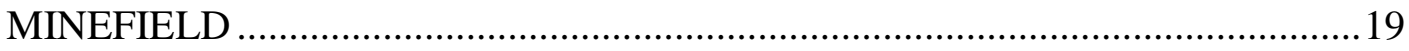

3.1 Preparation of Practice Landmines .................................................. 19

3.2 Preparation of the Test Minefields...................................................21

3.3 Preparation of the First Test Field ...................................................21 
3.4 Preparation of the Second Test Field .................................................24

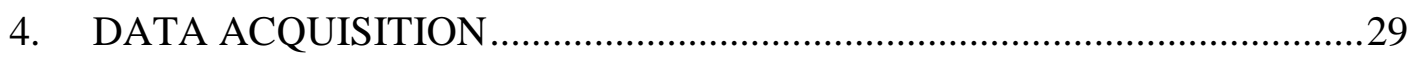

4.1 Cameras and Their Characteristics ....................................................29

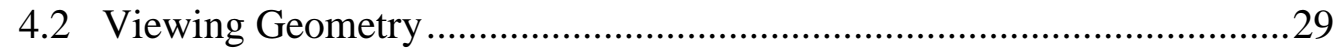

4.3 First Acquisition: One Day After Establishment of the First Minefield..31

4.4 Second Acquisition: Two Months After the Establishment of the First

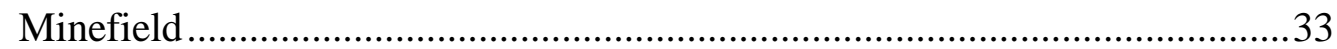

4.5 Third Acquisition from the Second Minefield .....................................34

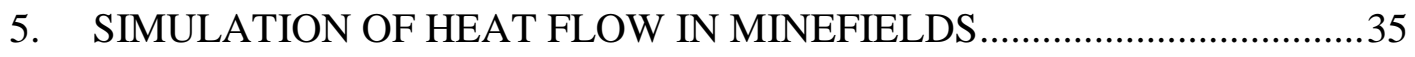

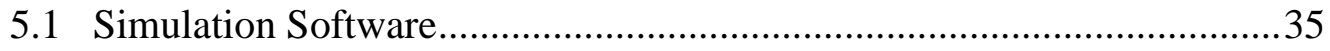

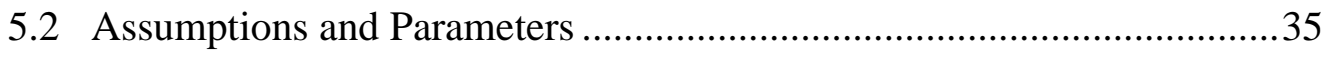

5.2.1 Boundary and Initial Conditions..............................................36

5.2.2 Figure 29 Bottom border temperature change when the soil depth is one meterSoil and PLs' Properties................................................... 37

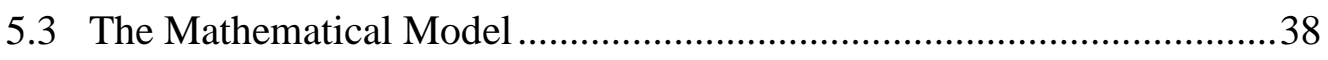

5.3.1 Physical Description of the Processes .........................................38

5.3.2 Soil-Surface Heat Flux .............................................................39

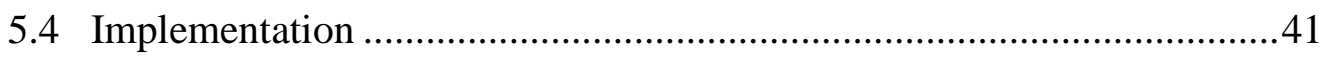

6. PROPOSED LANDMINE DETECTION ALGORITHM ...........................45

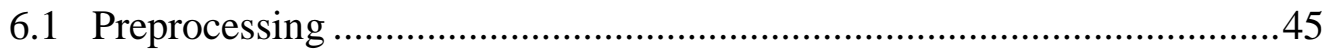

6.1.1 Noise reduction................................................................45

6.1 .2 Spatial Filtering .............................................................4

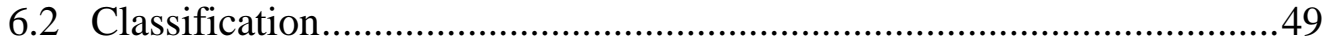

6.2.1 Preparation of the Training and the Test Sets............................49

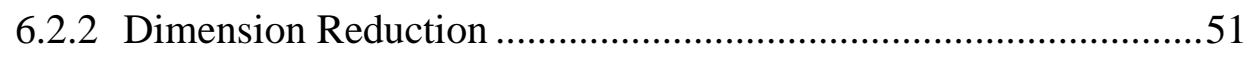

6.2.3 Supervised Classification ....................................................52

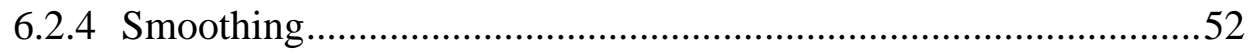

6.2.5 Post-Processing and Detection ..............................................52

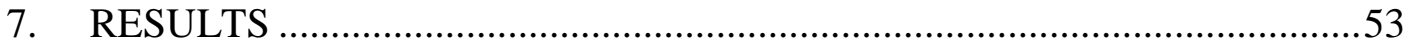

xii 


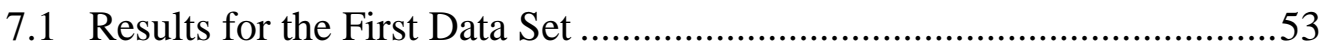

7.2 Second Data Sets Processing Results .................................................55

7.2.1 Thermal Image Time Series ...............................................55

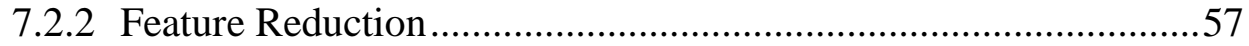

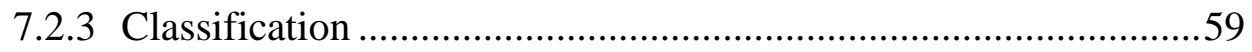

7.2.4 Post Processing and Automatic Detection..................................60

7.2.5 Performance Assessment ......................................................60

7.3 Third Data Processing Results ..................................................65

7.3.1 Thermal Image Series .......................................................65

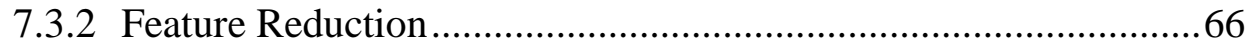

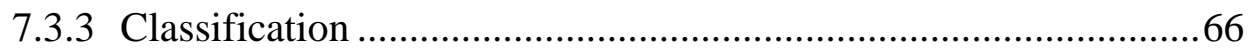

7.3.4 Post Processing and Automatic Detection .................................66

7.3.5 Performance Assessment .................................................... 70

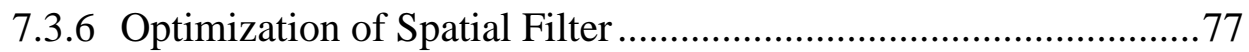

7.3.7 Performance Comparisons of the Daytime and Nighttime Image

Cubes ...................................................... 79

7.3.8 The Unfiltered (raw) and Filtered Image Cubes Performance

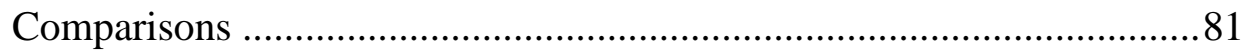

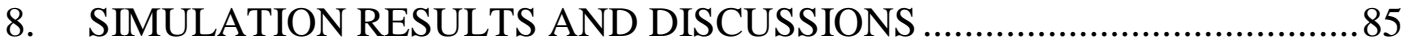

8.1 M48, and DM11 PLs Simulation Results with the First Test Area's

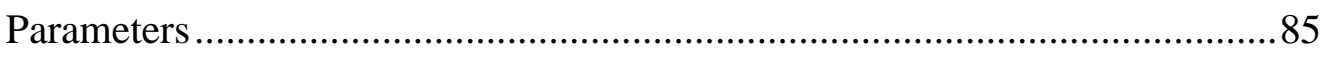

8.1.1 M48 Metal PL Simulation Results ..........................................8 85

8.1.2 DM11 Plastic PL Simulation Results .......................................92

8.2 DM11 PL Simulation Results with the Second Test Area's Parameters 100

8.3 Contributions of Simulation Results to the Study............................... 107

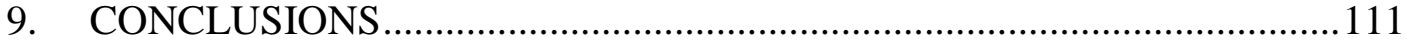

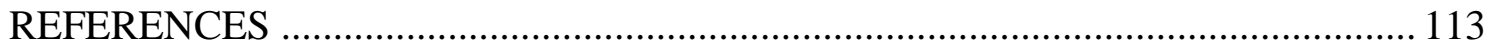

xiii 


\section{LIST OF TABLES}

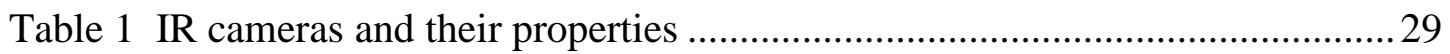

Table 2 Test areas' geographic and thermal properties ............................................... 36

Table 3 PLs' dimensions and assumed thermal properties........................................... 37

Table 4 Soils' assumed thermal properties and densities .............................................. 37

Table 5 The image cubes sizes, used cameras, and dates .........................................4 46

Table 6 The class names, sample sizes, and training set sizes...................................50

Table 7 The class names, sample, and training set sizes for second minefield data..50

Table 8 Detection / False Alarm Rates of the classifiers before smoothing ...............63

Table 9 Detection/ False Alarm Rates of the classifiers after smoothing...................64

Table 10 True Positive, False Negative and False Positive values..............................64

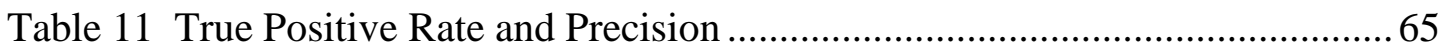

Table 12 Detection and false alarm rates chance with smoothing in..........................73

Table 13 Detection and false alarm rates chance with smoothing in ..........................73

Table 14 Detection/false alarm rates change after smoothing wrt to training sizes .. 74

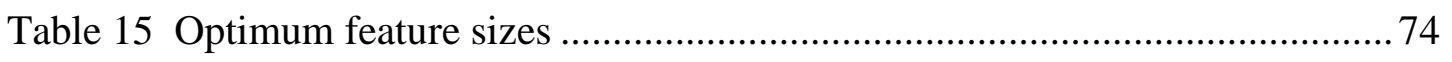

Table 16 True Positive, False Negative and False Positive values............................. 76

Table 17 True Positive Rate and Precision .............................................................. 76

Table 18 Rate results with various $R_{o}$ before smoothing classification results ..........77

Table 19 Rate results with various $R_{o}$ after smoothing classification results ..............77

Table 20 Sample-point numbers' influence on detection results ................................79

Table 21 Detection and false alarm rates with respect to imaging time of the day ...79

Table 22 Detection and false alarm rates comparisons of raw and filtered date ........82

Table 23 First test area based simulation solution ................................................... 100

Table 24 Simulation solutions for the second test area............................................ 106

Table 25 Detection results with respect to time intervals ......................................... 109 


\section{LIST OF FIGURES}

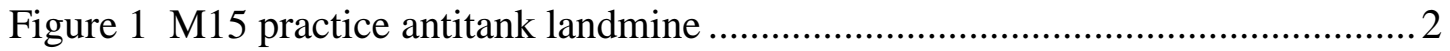

Figure 2 M16 metal practice antipersonnel landmine.......................................... 2

Figure 3 M14 plastic practice antipersonnel landmine .......................................... 3

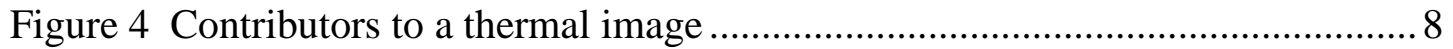

Figure 5 Hypothetical two-dimensional data' KLT transformation ........................... 9

Figure 6 An example of supervised classification result. The labeled pixels are represented by different colors ............................................................. 11

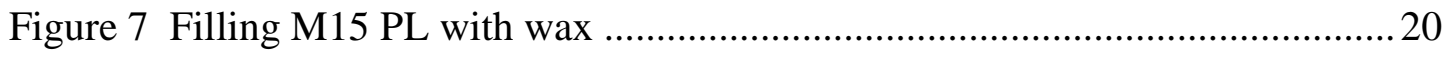

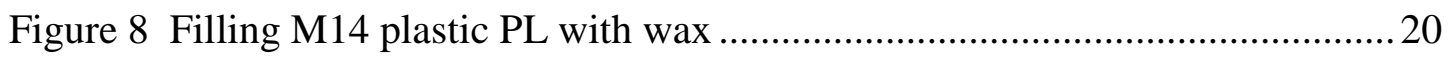

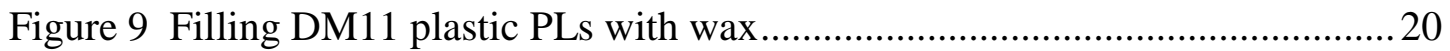

Figure 10 Filling the M2 landmine with metal bar .............................................2 21

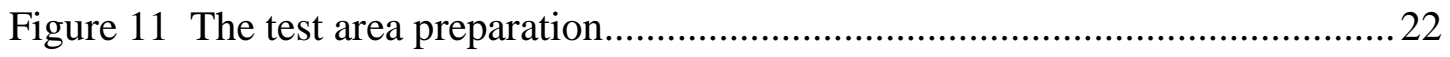

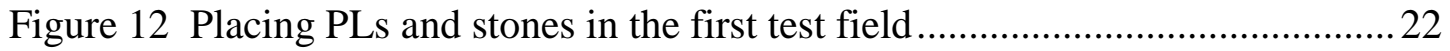

Figure 13 Layout of landmines in the test field .............................................. 23

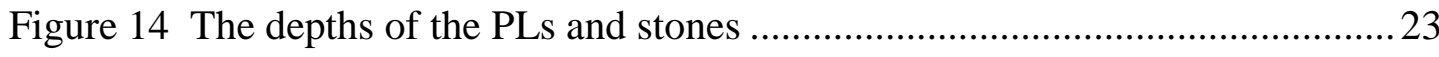

Figure 15 The PLs in the test area before burial ...............................................24

Figure 16 The final appearance of the minefield after all PLs and stones were

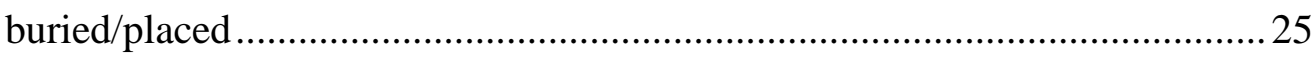

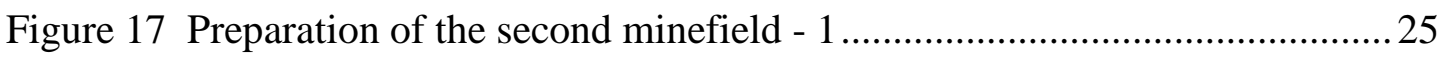

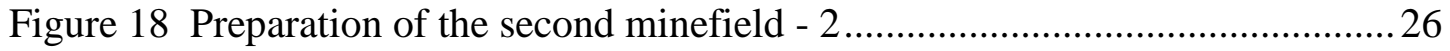

Figure 19 Layout of landmines in the second minefield .......................................26

Figure 20 The PLs in the second minefield before burial.....................................2 27

Figure 21 Final appearance of the second minefield .............................................2 28

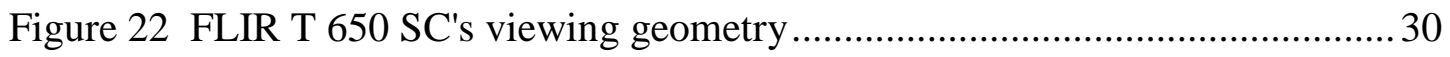

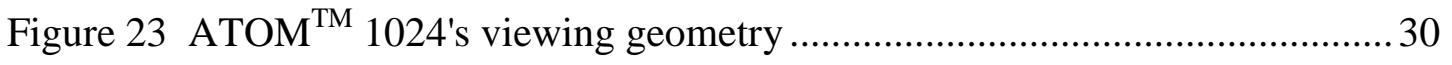

Figure 24 The second minefield camera viewing geometry .................................. 31 
Figure 25 The image captured on 8th of July 2015 at 11:10 a.m. ............................. 32

Figure 26 The image captured on ninth of July 2015 at $05: 10$ a.m. ........................ 32

Figure 27 The image captured at 2:00 a.m. by FLIR T 650 SC..............................33

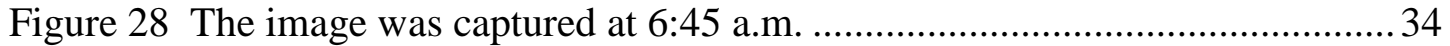

Figure 29 Bottom border temperature change when the soil depth is one meterSoil

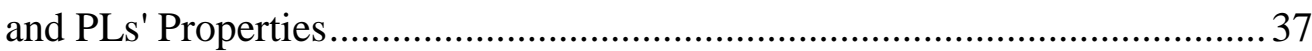

Figure 30 Heat transfer processes taking place at the air-soil interface and ..............38

Figure 31 The simulation geometry for M2 PL metal mine ................................. 41

Figure 32 The simulation geometry for DM11 PL plastic mine............................. 42

Figure 33 The meshed example of soil cross-section geometry .............................42

Figure 34 The soil surface' temperature distribution ......................................... 43

Figure 35 3-D the temperature distribution on the soil surface during the diurnal

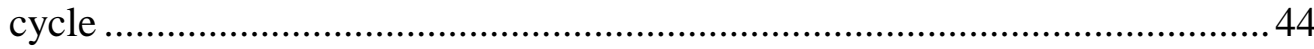

Figure 36 Temperatures distribution inside the soil .......................................... 44

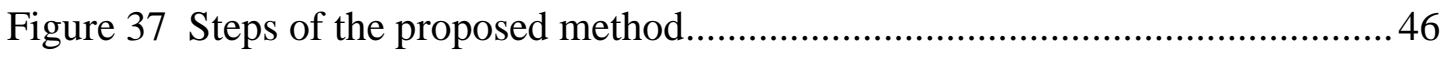

Figure 38 The image cube formation and noise removal process.......................... 47

Figure 39 The circular spatial filter's representation.......................................... 48

Figure 40 An example band of spatial filtered image captured by FLIR camera from

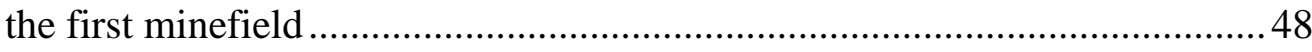

Figure 41 An example band of spatial filtered image captured by FLIR camera from

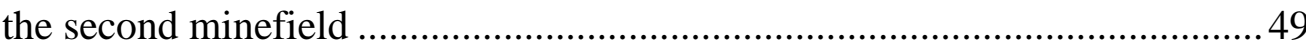

Figure 42 The second minefield's ground truth with the buffer zones .....................51

Figure 43 Training set formed version of ground truth ......................................51

Figure 44 The average behavior of pixels from each class as a function of time ......53

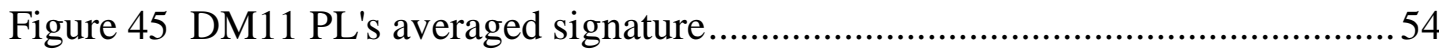

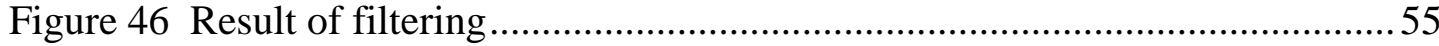

Figure 47 Diurnal thermal differences captured by ATOM ${ }^{\mathrm{TM}} 1024$......................56

Figure 48 Intensity differences captured by FLIR T 650 SC ................................57

Figure 49 Diurnal original thermal radiance change captured by ATOM $^{\mathrm{TM}} 1024 \ldots . .58$ 
Figure 50 Detection/False Alarm Rates vs. KLT feature sizes before smoothing the

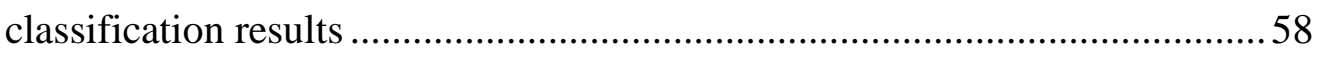

Figure 51 Detection/False Alarm Rates vs. KLT feature sizes after smoothing the

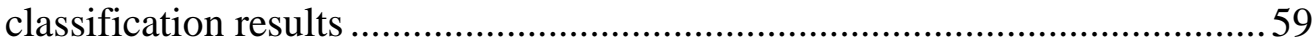

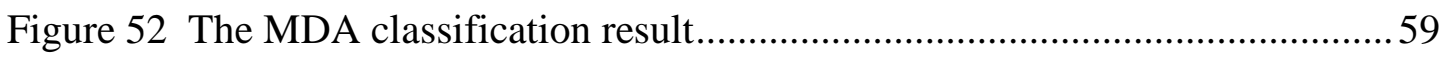

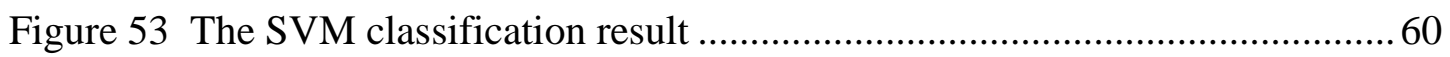

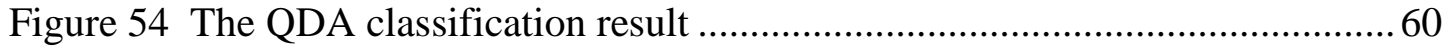

Figure 55 The smoothed QDA classification result............................................ 61

Figure 56 The MDA automatic detection result after smoothing ...........................61

Figure 57 The SVM automatic detection result after smoothing ............................62

Figure 58 The QDA automatic detection result after smoothing ............................62

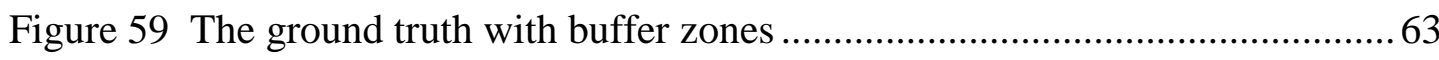

Figure 60 Diurnal thermal differences captured by FLIR ....................................67

Figure 61 Intensities differences captured by FLIR (9:00 p.m. to 06:45 a.m.). .......66 67

Figure 62 Original thermal radiance change for the $3^{\text {th }}$ data set .............................6

Figure 63 Detection/False Alarm Rates vs. KLT feature sizes before smoothing the

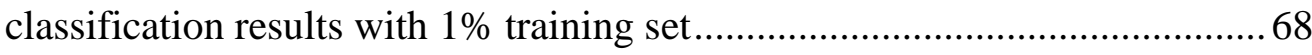

Figure 64 Detection/False Alarm Rates vs. KLT feature sizes after smoothing the classification results with $1 \%$ training set...............................................69

Figure 65 Detection/False Alarm Rates vs. KLT feature sizes before smoothing the

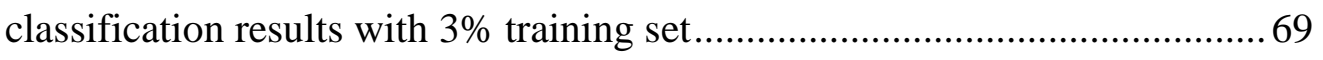

Figure 66 Detection/False Alarm Rates vs. KLT feature sizes after smoothing the classification results with $3 \%$ training set................................................ 70

Figure 67 The k-NN classification result before smoothing .................................. 71

Figure 68 The SVM classification result before smoothing ................................. 71

Figure 69 The MDA classification result............................................................. 71

Figure 70 The QDA classification result before smoothing ................................. 72

Figure 71 The smoothed QDA classification result .............................................. 72

Figure 72 The k-NN automatic detection result ................................................. 74 


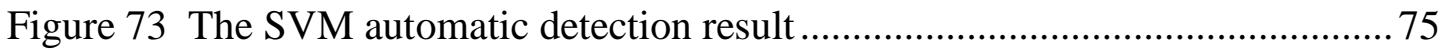

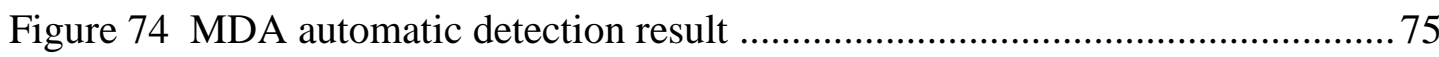

Figure 75 The QDA automatic detection result................................................. 75

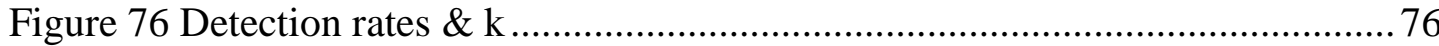

Figure 77 Detection/False Alarm Rates vs. KLT feature sizes before smoothing the classification results with $3 \%$ training set............................................... 78

Figure 78 Detection/False Alarm Rates vs. KLT feature sizes after smoothing the classification results with $3 \%$ training set................................................ 78

Figure 79 Detection/False Alarm Rates vs. KLT feature sizes after smoothing the classification results with respect to used data sets .................................... 80

Figure 80 The classification result of nighttime image cube $(m=11)$.................... 80

Figure 81 The classification result of daytime image cube $(\mathrm{m}=11)$...................... 80

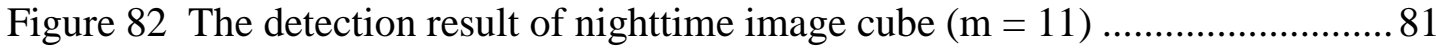

Figure 83 The detection result of daytime image cube $(m=11)$............................. 81

Figure 84 The classification result of nighttime raw image cube $(\mathrm{m}=12) \ldots \ldots \ldots \ldots . . .82$

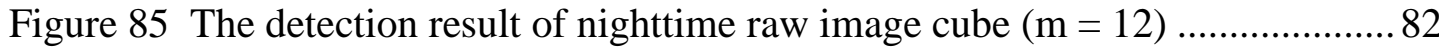

Figure 86 3-D temperature distribution on the soil surface during diurnal cycle

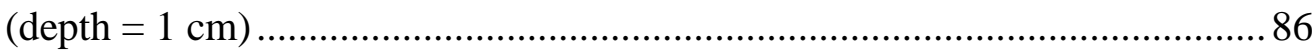

Figure 87 Cross-section of temperature distribution when the PL encountered soil surface, and soil surface's temperature differences are maximum................87

Figure 88 The image captured at 6 am with FLIR T 650 SC on 9 September 2015.87 Figure 89 Soil surface temperature and PL encountered soil surface/deep soil

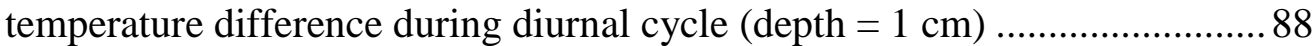

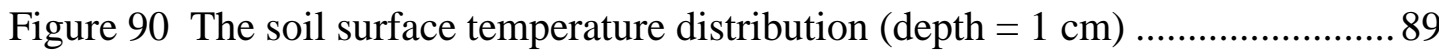

Figure 91 3-D temperature distribution on the soil surface during diurnal cycle

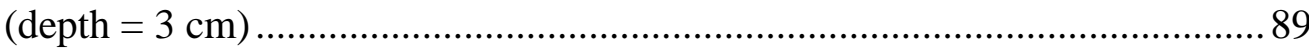

Figure 92 Cross-section of temperature distribution when the PL encountered soil surface, and soil surface's temperature differences are maximum.................90 
Figure 93 Soil surface temperature and PL encountered soil surface/deep soil

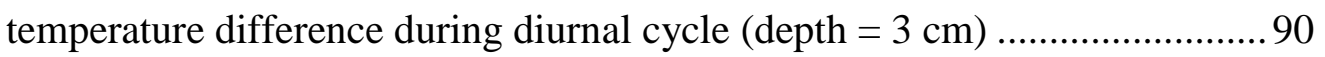

Figure 94 The soil surface temperature distribution $($ depth $=3 \mathrm{~cm})$..................... 91

Figure 953 -D the temperature distribution on the soil surface during diurnal cycle

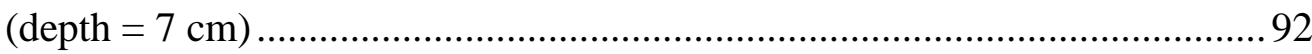

Figure 96 Soil surface temperature and PL encountered soil surface/deep soil temperature difference during diurnal cycle $($ depth $=7 \mathrm{~cm}) \ldots \ldots \ldots \ldots \ldots \ldots \ldots . . . . . .93$

Figure 97 Cross-section of temperature distribution when the PL encountered soil surface, and soil surface's temperature differences are maximum.................93

Figure $983-\mathrm{D}$ the temperature distribution on the soil surface during diurnal cycle $($ depth $=1 \mathrm{~cm})$ .94

Figure 99 Soil surface temperature and PL encountered soil surface/deep soil

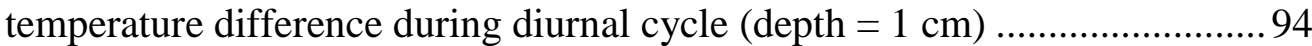

Figure 100 The soil surface temperature distribution $($ depth $=1 \mathrm{~cm})$.....................95

Figure 101 Cross-section of temperature distribution when the PL encountered soil surface, and soil surface's temperature differences are maximum................ 95

Figure $1023-\mathrm{D}$ the temperature distribution on the soil surface during diurnal cycle $($ depth $=3 \mathrm{~cm})$ .96

Figure 103 Soil surface temperature and PL encountered soil surface/deep soil

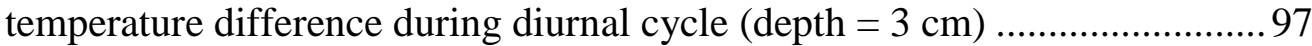

Figure 104 The soil surface temperature distribution $($ depth $=3 \mathrm{~cm})$......................97

Figure 105 Cross-section of temperature distribution when the PL encountered soil surface, and soil surface's temperature differences are maximum.................98

Figure 106 3-D the temperature distribution on the soil surface during diurnal cycle $($ depth $=7 \mathrm{~cm})$ .98

Figure 107 Soil surface temperature and PL encountered soil surface/deep soil temperature difference during diurnal cycle $($ depth $=7 \mathrm{~cm})$ 99

Figure 108 The soil surface temperature distribution $($ depth $=7 \mathrm{~cm})$ .99 
Figure 109 Cross-section of temperature distribution when the PL encountered soil surface, and soil surface's temperature differences are maximum................. 100

Figure 1103 -D the temperature distribution on the soil surface during diurnal cycle

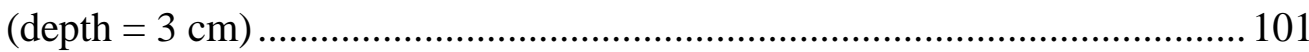

Figure 111 Diurnal temperature distribution of $3 \mathrm{~cm}$ buried PL ……….................. 102

Figure 112 Soil surface temperature and PL encountered soil surface/deep soil temperature difference during diurnal cycle $($ depth $=3 \mathrm{~cm}) \ldots \ldots \ldots \ldots \ldots \ldots \ldots \ldots . . . .103$

Figure 113 The soil surface temperature distribution $($ depth $=3 \mathrm{~cm})$.................... 103

Figure 114 Cross-section of temperature distribution when the PL encountered soil surface, and soil surface's temperature differences are maximum................. 104

Figure 1153 -D the temperature distribution on the soil surface during diurnal cycle $($ depth $=4 \mathrm{~cm})$ 104

Figure 116 Soil surface temperature and PL encountered soil surface/deep soil temperature difference during diurnal cycle $($ depth $=4 \mathrm{~cm})$......................... 105

Figure 117 Cross-section of temperature distribution when the PL encountered soil surface, and soil surface's temperature differences are maximum................. 105

Figure 118 The soil surface temperature distribution $($ depth $=4 \mathrm{~cm})$.................... 106

Figure 119 Images captured at 6:00 am from first and second test areas ................. 107

Figure 120 4:45 am - 7:45 am period object-based detection result ......................... 108

Figure 121 4:00 am - 7:15 am period object-based detection result ......................... 109

Figure 122 5:00 pm - 8:00 pm period object detection result.................................. 109 


\section{CHAPTER 1}

\section{INTRODUCTION}

\subsection{What are Landmines?}

Since landmines are cheap and easy-to-place lethal passive weapons, they are used frequently all over the world. Most of them have simple working mechanisms and after placing them, there is no need for maintenance. In addition, landmines are much cheaper than having personal that can produce same effect as landmines can do on targets. Because of these advantages, there has been great demand on landmines. Thus, many counties have been manufacturing landmines as suppliers, and other country or groups have been purchasing as users. Therefore, there are a huge number of landmines to be detected and cleaned.

The landmines' actual number is not known [1] and it will not be known either. Most probably, this number is getting higher and higher every day. Therefore real number of landmines actually is not important at all [2], the important thing is antipersonnel landmines cause hundreds of civilian casualties every year, in addition to preventing use of these areas for agriculture or other purposes.

A landmine contains explosives that can be triggered by people, vehicles, or animals via a mechanism [3]. The landmines can be divided mainly into two categories according to their targets: antitank landmines and antipersonnel landmines. The targets of antitank landmines are mainly military vehicles, and those of antipersonnel landmines are civilian or military people. Antipersonnel landmines can also be divided into two 
categories according to materials that they consist of, namely, metal antipersonnel landmines and plastic antipersonnel landmines. Antitank landmines mostly have metal shell and they contain quite large amount of explosives. Figure 1 shows an M15 practice antitank landmine. On the other hand, metal antipersonnel landmines mostly consist of fragmentation, and other metal contents. An M16 metal practice antipersonnel landmine is shown in Figure 2. Because of high amount of metallic content, antitank and metal antipersonnel landmines can be found by metal/conventional detectors. However, metal detectors have to be operated by an expert while detecting landmines. Lastly, antipersonnel plastic landmines have plastic shells and contain mainly explosives. An example plastic practice antipersonnel landmine is shown in Figure 3. The dielectric properties of plastic and soil are similar to each other and hence this makes detection of them virtually impossible using this technique. Therefore, metal detectors may not find a plastic landmine that contains little or no metal content [3], [4]. The plastic landmines may be masked by their background as well.

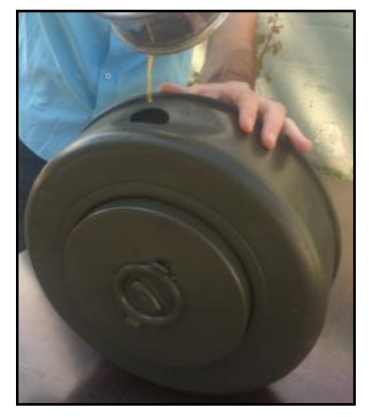

Figure 1 M15 practice antitank landmine

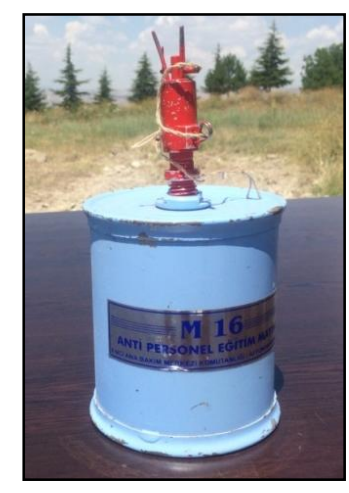

Figure 2 M16 metal practice antipersonnel landmine 


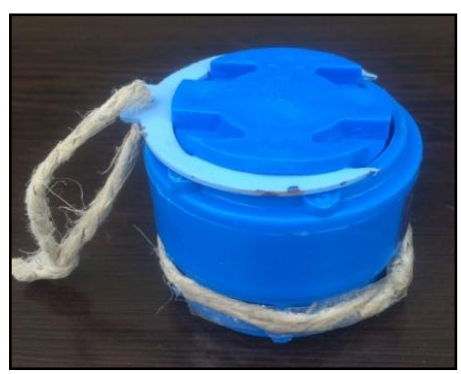

Figure 3 M14 plastic practice antipersonnel landmine

\subsection{Landmine Detection and Minefield Cleaning}

Approximately US $\$ 1.093$ billion of fund was used [5] only in 2013 in order to reduce or lessen negative effects of landmines. A landmine's cost of production is around $\$ 3$, but disposal of the same mine costs between $\$ 300-\$ 1000$. Thus, landmines cause a huge cost, not only for placed countries, but also for international community as well. A huge number of land mines have been cleaned up to now, but a few multiple of that number have been planted in the same time interval. In addition to the clearance cost, there are human costs, care cost [6]. Because of these reasons, mine detection has become a very important requirement.

The principle of Infrared Red (IR) radiation is that landmines create an observable temperature changes on the soil surface because they have different thermal properties than other materials in the same medium [7]. Therefore, the landmines might be detected with accurate measurements, good modeling of the physical process and a collection of image processing and pattern recognition techniques. The proposed method, which is given in this thesis, depends on passive IR. Detailed explanation of the method is presented in Section 2.

\subsection{Problem Statement}

There are many detection systems but none of them is reliable enough to detect landmines without missing a fraction. The United Nations landmine clearance standard for detection rate is $99.6 \%$ for humanitarian demining [8]. The standard is too high and 
essential. In addition to reaching this standard, safety during mine detection and cleaning is another necessity. The problem is that most of the methods need an operator, who detects the landmines in situ, or a tool, which is needed to detect landmines, or frequently both, to enter to the minefield. As mentioned before, neither people's life, nor landmine detection tools are cheaper than a landmine.

In addition to the necessity of detecting landmines without entering minefield, the minefield itself must be detected in the first place. Many landmines have been not marked after being planted. The minefields, which are built either by military personnel or civilian, most of the times are not signed because their aim is either to damage counterparties or to notice their approach in advance in order to be ready for a counter operation. Border minefields are signed intentionally to deter trespassers.

In order to detect landmines, minefield itself has to be found in the first place. Following that, all mines should be detected without putting lives at risk. Therefore, an automatic safe landmine detection system that does not need an operator or any detection tools to enter the minefield is needed.

\subsection{Advantages of the Proposed Method}

To sum up, methods are needed to detect, first, the minefield's approximate location by finding one or more landmines, because most of the time the minefields locations are not known as mentioned previous section. Then detecting the landmines in the minefield is necessary to clean the entire minefield. In addition, detected landmines should be classified according to their types, like antitank, metal, and plastic landmines by a method.

After detection of landmines location with proposed method, an operator has to enter the minefield to clean the landmines. The operator has to use detectors to find landmines in their known location. Metallic landmines can be found easier than plastic landmines with these detectors. Therefore, it is important to distinguish landmines' content. In addition, metal antipersonnel landmines give much more harm than plastic antipersonnel 
Thus firstly metal landmines should be found, which also means finding minefield most of the times, after that plastic landmines should be found. Therefore, it is important to determine landmines' types in advance to be able to plan a safe and effective cleaning operation.

\subsection{Contribution of the Thesis}

To detect landmine types and their location in the minefield without entering it gives the cleaning personnel the opportunity to estimate the minefield location, and the minefield map that shows the landmine placement pattern. After getting this information about landmines, cleaning of minefield would be much safer and faster when compared to conventional methods.

This thesis aims to contribute automated landmine detection systems that rely on thermal cameras, which can be installed on the borders of the area obliquely or can be carried on board of an unmanned air vehicle. Different from earlier work, proposed method consists of a two-step algorithm for detection landmines in time series images. At the first step, a specific spatial filter is applied on the image series and the filtered image series is classified with various supervised classifiers. At the second step, the classified pixels are combined using connectivity analysis to detect mines. Hence, the algorithm described not only detects mines but also classifies them into mine types. The method is strictly image-based and does not depend on any thermal model; however, a thermal model is also established to verify various assumptions. The classification and detection performances of the algorithm are shown on multiple sets of real data. 


\section{CHAPTER 2}

\section{BACKGROUND INFORMATION AND LITERATURE REVIEW}

\subsection{Heat Flow on the Soil Surface}

IR cameras can detect the radiated energy from the soil surface [9], [10]. The atmosphere and the soil thermal emissivity modify the energy to some extend recorded by a sensor. The atmosphere reduces the energy illuminating at the ground surface, and it adds scattered path radiance to the signal detected by the IR sensors [11]. If there were no atmosphere, the measured signal by the IR sensor would be a function of the energy from the sun, reflectance, and emission properties of the ground [12]. Direct sunlight, aerosol-scattered sunlight, thermal emissions from the air reflected by the ground, and thermal emissions from the soil are the contributors to a thermal image of the ground. These mentioned components are illustrated in Figure 4 [13]. Since the illumination by direct light is not uniform due to the roughness of the surface and shadows, IR clutter is formed on the surface. This is not noise, but an unwanted slowly changing contribution to the desired signal and hence it makes detection more difficult. The total radiance $\left(L_{R}\right)$ received by the IR sensor is given in the formula:

$L_{R}\left(W m^{-2} s r^{-1}\right)=\rho L_{S U N}+\rho L_{S K Y}+\epsilon L_{T}$

where $\rho$ is the surface reflectivity in the thermal band, $\epsilon$ is the surface emissivity, $L_{S U N}$ is the radiance because of the sunlight, and $L_{S K Y}$ is the radiance because of the sunlight scattered by particles and molecules in the earth's atmosphere and thermal radiation from the warm atmosphere. Lastly $L_{T}$ is the surface thermal radiance.

The surface emissivity for an opaque body is $\epsilon=1-\rho$. If we make the definition 
$L_{S S}\left(W m^{-2} s r^{-1}\right)=L_{S U N}+L_{S K Y}$

then $L_{R}$ is turns to be

$L_{R}\left(W m^{-2} s r^{-1}\right)=(1-\epsilon) L_{S S}+\epsilon L_{T}$.

Note that emissivity is around 0.95 for most natural materials and hence reflectance is very small. Equation (3) is presented as a well-known problem in IR detection by Sendur and Baertlein [13].

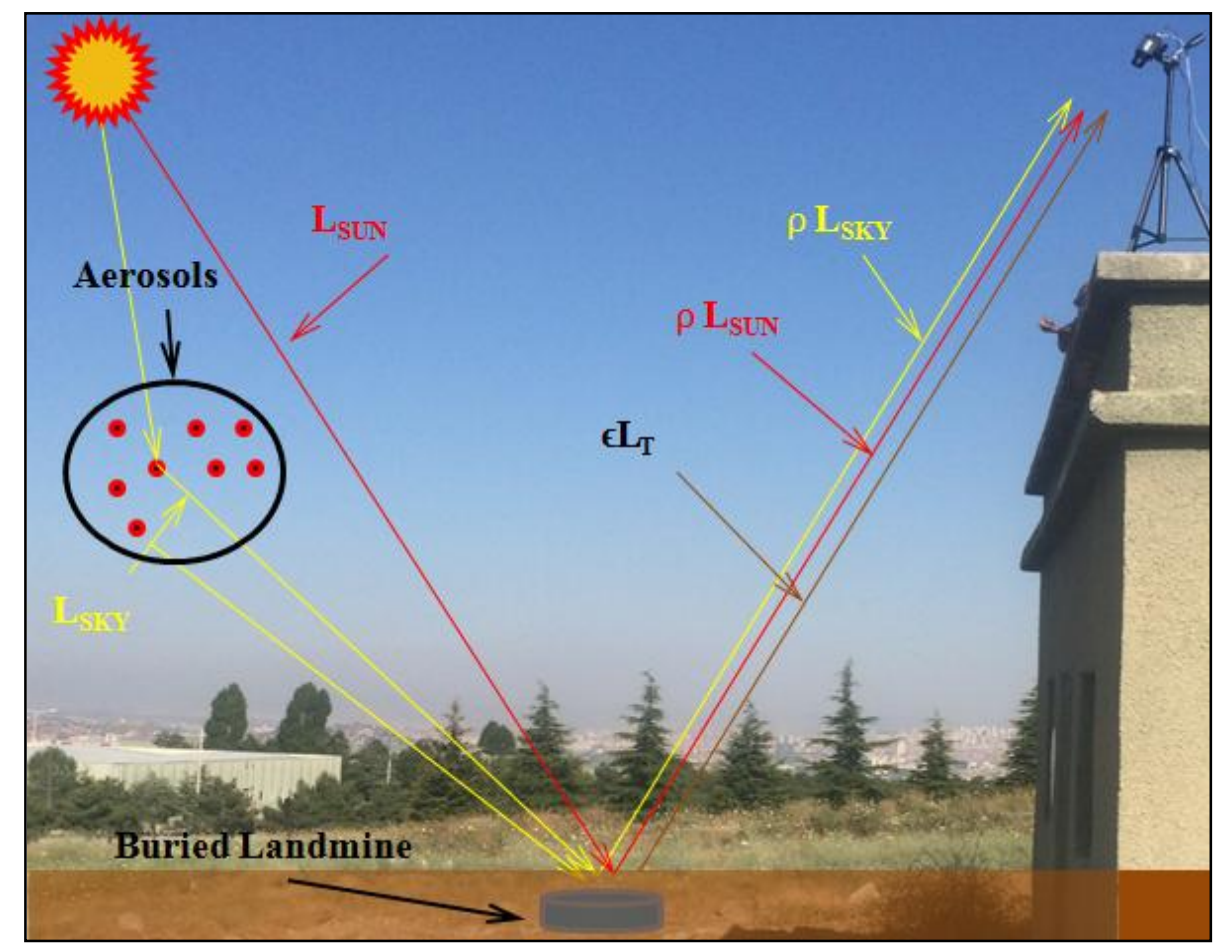

Figure 4 Contributors to a thermal image

\subsection{Karhunen-Loève Transformation (KLT)}

There are various algorithms in order to select features or combination of features. One of the most commonly encountered algorithms in remote sensing is Karhunen-Loève, Hotelling, or in other saying, the Principal Components, Transform. The aim of the 
transformation is to create the most discriminating band combinations and discard the rest of data, so that feature reduction can be carried out by transforming to the new set of axes that are uncorrelated [12]. This reduction can also be possible for image series, because images series have high degree of redundant information in their raw form. This reduction enables storing data efficiently as well [14].

Figure 5 illustrates hypothetical two dimensional, two class data illustrating lack of separability in original data but separability along the principal component. Black axes represent the original coordinate system and red axes represent principal axes. The transformation allows representing the multidimensional data in a lower dimensional space. Therefore, KLT reduces the space and hence the computational complexity during the classification process [15].

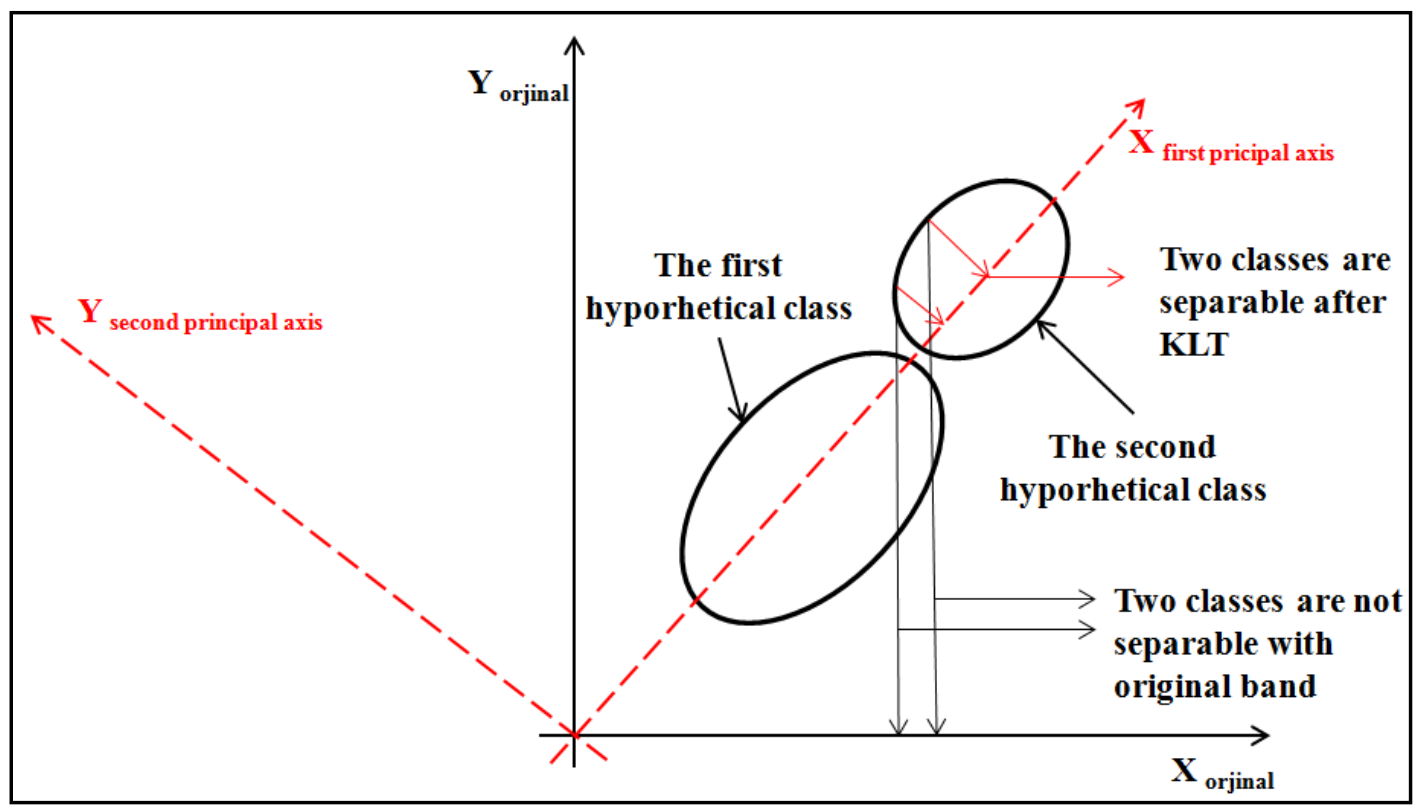

Figure 5 Hypothetical two-dimensional data' KLT transformation

The time-varying four-set of image sequences IR data used in this study consist of 48, 96, 39, and 96 images respectively. Therefore, data sets need to be reduced for minimizing the time of process and allowing programs process the data. 


\subsection{Classification}

The aim of image classification is to sort all pixel vectors into PLs and soil automatically. Normally, multispectral data are used to carry out the classification, but thermal image time series are used in this study [11]. Every image in the image cube can be thought as a different spectral band.

\subsection{Supervised Classification}

Training set can be named as representative signature or interpretation key that describes the spectral attributes for each feature type. There is an analyst who decides or selects the training fields/representative signatures and these training sets are used by the algorithms to characterize feature types in supervised classification method. This process is frequently termed training [11], [12]. In this study, training set pixels were selected randomly from predefined class locations using the ground truth. Some classification algorithms compare each pixel vector to each representative signature in the training set numerically and labels pixels in the most likely category using predefined decision rule. A few pixels are not labeled because of insufficient similarities to any training data set, and they are labeled as unknown [11].

The training process is the major step of supervised classification. If representative signatures do not represent the classes good enough, this causes poor classification. Therefore supervisor have to be experienced in marking the training sets.

The result can be presented as either a thematic map or a table. [12]. In this study, classification results are shown as thematic maps. Figure 6 is given as an example of classification thematic map, where labeled pixels are represented with colors shown in the legend. 


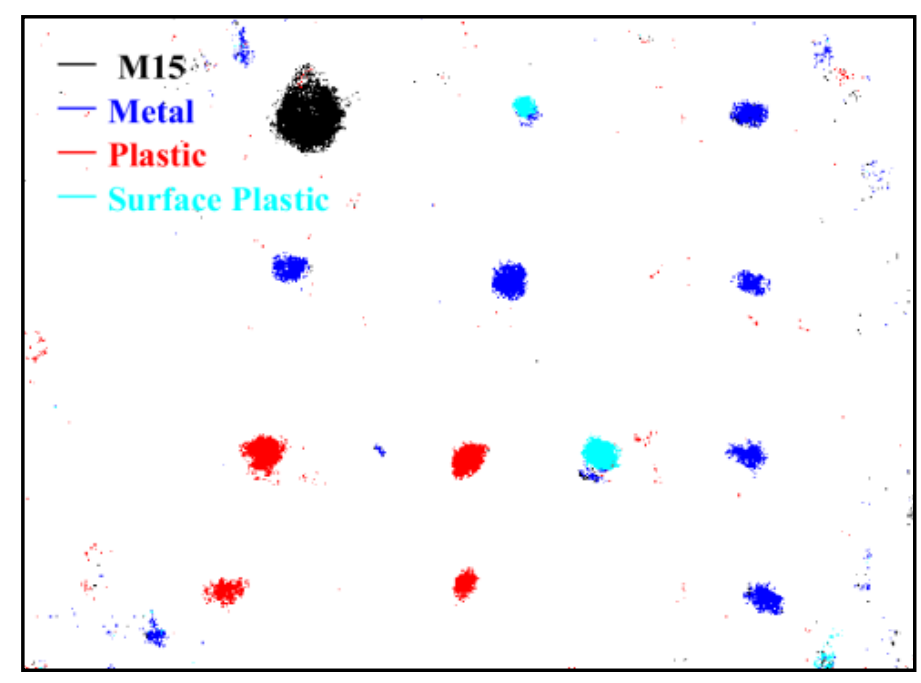

Figure 6 An example of supervised classification result. The labeled pixels are represented by different colors

\subsection{Unsupervised Classification}

Unsupervised classification does not use training data for classification. The algorithms examine the unknown pixels in data and aggregate them into a number of symbolic classes based on clusters in the feature values.

The symbolic classes resulting from unsupervised classification are spectral classes and they represent only natural groups according to image values. Therefore the analyst, who does not get involved in the classification process at the beginning, intervenes in the posteriori identification when he/she attaches information class labels to the segments established by clustering [11], [12].

\subsection{Landmine Detection Methods}

Even though there are many landmine detection systems, the most frequently used ones are sensor-based systems. The magnetic field-based metal detectors have been widely used for landmine detection [16]. The other sensor-based systems are Infrared (IR), Ground Penetrating Radar (GPR) [4], and Ultra Sound Sensor (US). These methods have some advantages and disadvantages. 
GPR is one of the most frequently used sensor in order to detect landmine and unexploded ordnance (UXO). GPR is operated just above the soil [17] and there are some examples in use like Husky, which had been created for humanitarian landmine cleaning in South Africa. These vehicle-mounted mine detection systems are being used in Afghanistan, Iraq and other places for military purposes. The GPR's transmitter sends an electromagnetic signal into the soil, and detects the reflected signal at the receiver [18], [19]. The transmitter sends signals continuously and the receiver collects backscatter signals from discontinuities. The depth of object can also be given by GPR, but its performance depends on humidity of soil. Very dry soil's electrical contrast is less than plastic landmines so GPR cannot find plastic landmines in dry soil [7]. Even though the GPR is inbuilt in armored vehicles, when it encounters missed landmines or UXO, the consequences of explosion could be harmful.

These types of tools are quite expensive, and they are not easy to operate because of their sizes. On the other hand, IR detection systems are quite cost-effective, and operationally friendly. In addition to that, the radar-based technology uses microwave part of the spectrum and small landmines or Improvised Explosive Devices can be missed because of the spatial resolution limit imposed by the corresponding wavelengths [16]. These misses can lead to destruction of the tools or even can be fatal for the operator.

Metal detectors are most widely used tools for mine detection. Metal detector produces a magnetic field, and this field interacts with the electrical or magnetic properties of the target. This interaction causes generation of a new magnetic field. The detector's receiver detects the new magnetic field that signals an object that contains metal. However, in order to detect landmines, the landmine has to contain considerable amount of metal. The metal detectors cannot detect low metal-content mines [16]. Especially antipersonnel landmines are difficult to find because they have small sizes and plastic ones have little or no metal content [3]. The metal detector cannot distinguish landmine and metal debris as well [7]. If the soil contains much debris or other metal elements, 
metal detector cannot distinguish landmines from other metal contents and hence gives false alarms continuously.

In addition to these factors, the operator of the detector has to enter to the minefield and keep metal detector near the soil surface, therefore operator has to be alert and has to concentrate all the time when he/she is in the minefield. Thus, the metal detector's detection accuracy actually depends mostly on operator's performance.

Buried landmine-sized objects cause measurable changes in the local elastic properties of the ground. These changes can also be detected by acoustic probing. This theory dates back to the 1950s [16]. The principle of landmine detection with the ultrasound is similar to that of the GPR's principle. The frequencies of ultrasound waves are much lower than those of the GPR. The transmitter emits the signal and the receiver collects backscattered signals [20]. While GPR signal do not make any physical disturbance in the medium, sound wave creates mechanical disturbance of molecules [20].

\subsection{Thermal Infrared in Landmine Detection}

While introductory information is given in Section 1, detailed information about thermal infrared in landmine detection is given in this section.

IR working principle is based on the fact that, different objects can have different thermal characteristics [7], i.e., thermal conductivity and heat capacity. The landmines can be thought of as unnatural volume for flow of the heat inside soil. This might cause specific spatiotemporal thermal pattern on the surface of the soil, which can be detected by IR imaging systems [21]. According to Santulli [22], IR-based detection systems depend mainly on soil surface conditions, soil nature, climatic variations, buried objects characteristics, their positions and lastly on the thermal excitation. When all these factors are handled appropriately, IR thermography is a noteworthy detection tool for detecting buried objects.

If these spatiotemporal thermal patterns occur because of landmines, it is called the volume effect. On the other hand, if they occur because of disturbed soil it is called the 
surface effect [21]. We experienced that the surface effect is detectable during only a short time interval after planting. During this period, the thermal contrast is quite distinguishable [20]. IR system may detect these anomalies as an evidence of landmines [23].

According to Kasban et al. [7] IR images do not need too much preprocessing, and this system can work with passive (natural) or active (human made) heat sources. However, it might be affected by weather conditions and humidity of the soil. Soil moisture contributes positively to a non-metallic landmine's thermal signature and improves detection rate; on the other hand, it reduces the detection rate of metallic landmines because of shifting thermal characteristics with moisture [24].

Deeply buried objects could not be detected with IR sensors [25]. The maximum limit of detecting landmines by IR is found to be around $10 \mathrm{~cm}$ [21]. Khanafer and Vafai [26] worked on imaging buried mines under three different soil surface conditions. According to their conclusion, mines that are buried moderate depths in the soil do not produce a direct signature.

There are similar studies published on detecting landmines using IR sensors. Hong et al. [27] monitored areas that contained buried anti-tank mines and analyzed surface temperature changes during diurnal cycle to compare various soil textures and soil moistures. According to their analysis, a cyclic behavior of landmines' thermal signatures can be predicted except for silt loam soil. Paik et al. [20] used 24-hour IR image time series in their studies. They used Karhunen-Loève Transformation (KLT) to reduce the size of the data, and applied three different methods to segment landmines. They enhanced the image/images using gray scale morphology. After that, a markerbased watershed algorithm is applied on the data for segmentation with these three methods. Ajlouni and Sheta [6], [28] presented landmine detection using KLT and watershed segmentation. In their studies, they offer nighttime image series from 20:00 to 01:00 with 30 minutes time interval. According to them, the images that are taken in the morning and afternoon time have redundant information. Therefore, they used night 
image series and KLT, which reduces number of images and hence time, required to process the data.

Nguyen et al. [29] worked on a three-dimensional thermal model for landmine detection problems. Martínez et al. [30] developed a three-dimensional thermal model to study the effect of landmines in bare soil. They worked on low or no metal content landmines. They modeled thermal behavior of soil with known boundary conditions. After that, they proposed an iterative method to classify the data. This iterative method gives the nature and depth of the objects. Sendurd and Baertlein [31] developed thermal radiometric model. They used a finite element method in order to describe the thermal phenomena. They used a $25 \mathrm{~cm}$ anti-tank mine stimulant and a virtual sensor which is assumed to be a LWIR camera operating near the $10 \mu \mathrm{m}$ wavelength. Additionally, they incorporated surface roughness into their thermal and radiometric models in order to take selfshadowing of the surface into account because of the soil surface topology. According to the authors, the surface temperature over the mine is cooler at dawn and the surface is hotter during the day. Lastly, during the night the layer of the soil above landmine is cooler. In addition, they represent the spectral differencing concept and they developed a detection algorithm based on pattern recognition principles in a different study [31]. They used weighted difference of visible and IR images from the same scene in order to remove the reflected radiance from the warm atmosphere to decrease the clutter resulted from reflected light. According to authors, there is a tradeoff between decreasing clutters and increasing mine signature [13]. Khanafer et al. [9] examined how the thin outer case made of metal and the air gap left above the buried antipersonnel and antitank mines affect the IR images. They used finite element method (FEM) to describe the thermal phenomena. They simulated buried antitank mines with and without a thin metal outer case, and surface/buried antipersonnel mines. In order to analyze top air gap effect, they simulated antipersonnel mine with top air gap as well. The simulated mines had TNT thermal properties in the model. According to their results, thin metal outer case has significant effect on the temperature distribution because of an appreciable difference in the thermal conductivity between the metal case and the TNT. The top air gap has a 
more noticeable effect on depth wise temperature variation within a given time cycle because of air gap's low thermal conductivity compared to soil. In addition to that, their results show that surface mines produce larger temperature extremes than buried mines. Thành et al. [32] introduced and validated a 3-D thermal model for detecting landmines in outdoor minefield data sets. They proposed a finite-difference approximation of generalized solutions of the model. Besides, they proposed methods to estimate the bare soil and air-soil interface thermal properties. They confirmed their estimated soil parameters by comparing the simulations with real data sets. They [33] also developed a method that gives buried objects' thermal diffusivity, depth, and size. In the first step, they presented a method that could detect the landmines. This method depends on thermal differences on the soil surface caused by buried objects. In the second part, their proposed method finds the thermal diffusivity, depth, and size of buried objects using the inverse problem setting. Three-dimensional simulations have been developed in order to model passive IR signature of land mines that are buried or placed on the soil surface using the (FEM). Nguyen et al. [10] proposed a two-step method in their review study. At the first step, they found soil temperature using their new thermal model provided by soil and buried object thermal properties. At the second step, detected objects are classified using proposed enhanced inverse problem setting. They referred the second step as inverse problem setting for landmine detection. They estimate buried object's depth, shape, and its thermal diffusivity with their two-step method. Muscio et al. [34] proposed a method that enables reproduction of thermal properties of outdoor conditions with reduced size of data and compressed time. They generated a generalized formula for this purpose. They imaged the built-in test area during eight and six hours with two hours period. They used a binary reduction algorithm to detect mines. However, according to their remarks this algorithm did not work well because of its noise sensitivity.

In our study, we used three to four hours sunset and sunrise data besides nighttime data that is around 10 hours. In addition, a simple yet effective circular spatial filter, whose shape is similar to landmines' shapes, is applied on the data. The algorithm both detected 
and recognized the landmines and the performance of the complete system is quantitatively determined.

Systems that fuse data from multiple sensor types have been offered as well. Nath and Bhuiyan [4] proposed a geometrical feature-based fusion system that consists of IR and GPR. According to the authors, false alarm rate was decreased in their fusion-based system and the system is an effective technique for detection and classification of antipersonnel landmines. Bhuiyan and Nath [35] also mentioned three different fusion methods which are data-level, feature-level and decision-level fusion. In order to get higher detection rate, shorter detection time and less false alarm rate, they propose these fusion methods. 


\section{CHAPTER 3}

\section{PREPARATION OF PRACTICE LANDMINES AND THE TEST MINEFIELD}

In this section, preparation of the practice landmines (PLs) and the test minefields that are used in the study are explained.

Two minefields were constructed and four sets of data were captured using two different cameras on three different dates. Second minefield was constructed to evaluate proposed algorithm performance with 20 DM11 PLs.

\subsection{Preparation of Practice Landmines}

The landmines that are used in this study do not contain any explosives; however, they should represent actual mines' geometric and thermal properties. In order to represent frequently encountered landmines, M15 antitank, M16, M2, and M48 metal, DM11, and M14 plastic PLs were used in our study. M15 and plastic mines were filled with wax that represents explosives' thermal characters [34]. Images from the procedure of filling M15 PL, M14, and DM11 with wax can be seen in Figure 7, Figure 8 and Figure 9, respectively. Real M2 landmines contain $60 \mathrm{~mm}$ mortar shells. Therefore, M2 PL was filled with metal content (Figure 10).

M14 plastic landmine's radius is only $5.6 \mathrm{~cm}$, which is one of the smallest landmines, and it contains little metal content. Because of this reason, it is accepted as nonmetallic landmine. Therefore, M14 plastic PL was intentionally used in this study in order to see IR response to nonmetallic, small plastic landmines. The aim is to see whether buried M14 and DM11 PLs can be detected by the proposed method or not, because one of the 
biggest problem is buried nonmetallic small plastic landmine detection. If the proposed algorithm can detect M14, most probably all landmines that are plastic nonmetallic and small can be detected by the proposed algorithm as well.

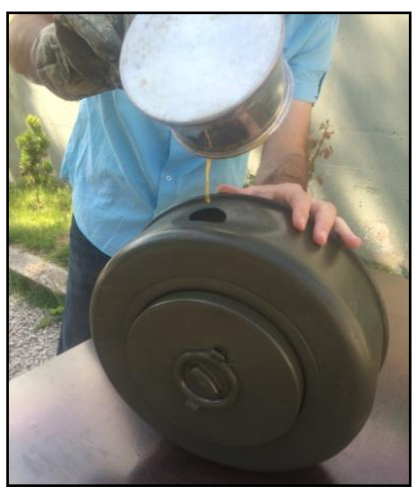

Figure 7 Filling M15 PL with wax

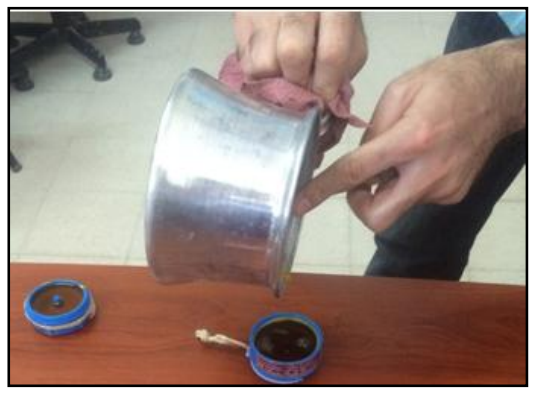

Figure 8 Filling M14 plastic PL with wax

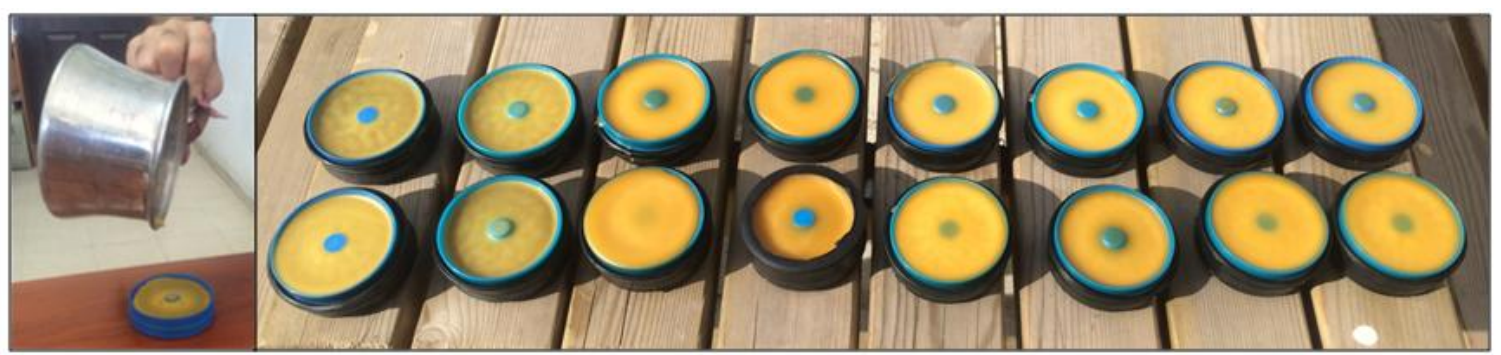

Figure 9 Filling DM11 plastic PLs with wax 


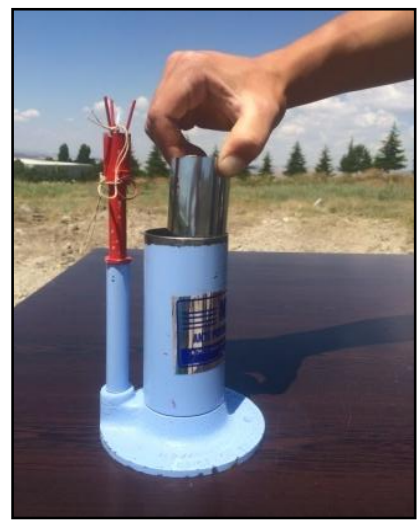

Figure 10 Filling the M2 landmine with metal bar

\subsection{Preparation of the Test Minefields}

The experiments were carried out in two different safe zones. The soil types are clay and clay loam, respectively. The minefields' locations were selected according to their thermal appropriateness. There are not any obstacles, which can cast shadow on the selected test area at these locations. However, we have seen at the end of the study that nighttime images are better than daytime images and sufficient for detection, therefore partial shadowing is not a major problem.

\subsection{Preparation of the First Test Field}

First landmine was established in July of 2015. The clay dry soil surface was cleaned from sparse vegetation. Then, a $2.2 \times 2.2 \mathrm{~m}$ area was dug $50 \mathrm{~cm}$ deep and all objects like stones, metal debris etc. in the $50 \mathrm{~cm}$ deep soil was cleaned. A stone with a shape similar to that of an M14 and another stone whose shape was similar to DM11 were buried in known locations in order to see their effect on detection accuracy of the proposed algorithm. The area dug can be seen in Figure 11. Thirteen PLs and two stones were used in total. Eleven PLs and two stones were buried and two plastic PLs that were a DM 11 and an M14 were located on the surface of the minefield. 


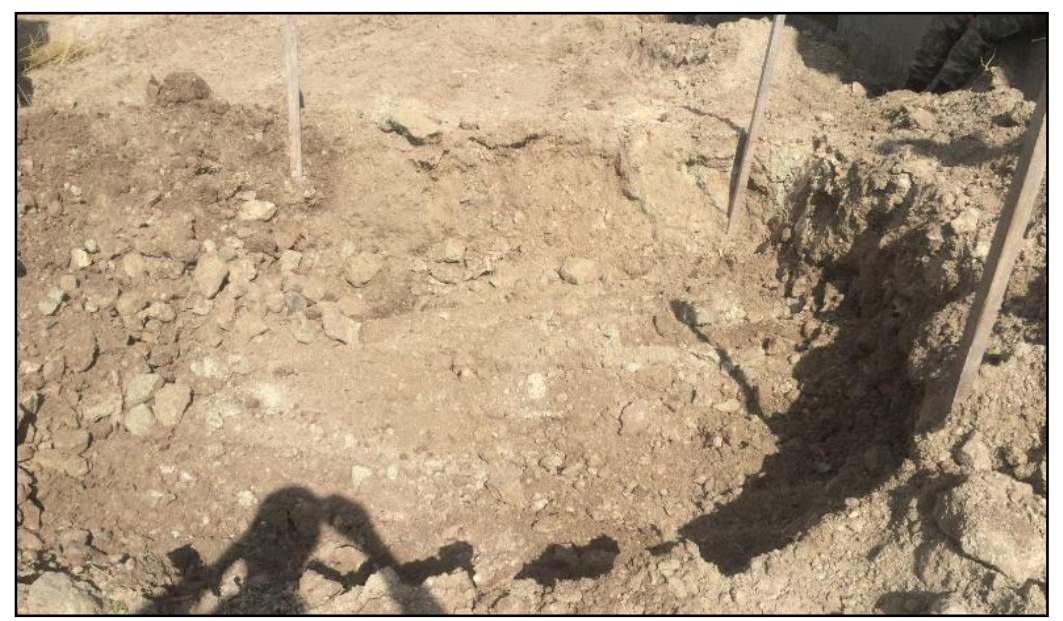

Figure 11 The test area preparation

The PLs were planted apart from each other to prevent thermal interaction among them. Figure 12 shows the placing of PLs and stones. The layout of the minefield is given in Figure 13 along with the depths that mines are buried.

The depths of PLs were selected according to the most encountered depths in real minefields. Therefore, the landmines were buried at different depths. For example, M15 anti-tank mine should be buried $2 \mathrm{~cm}$ deep from the soil surface [36]. All the visible area is dug before burial to avoid any disturbed earth effect in the images at the locations of mines. Figure 14 shows the placing of landmines at different depths.

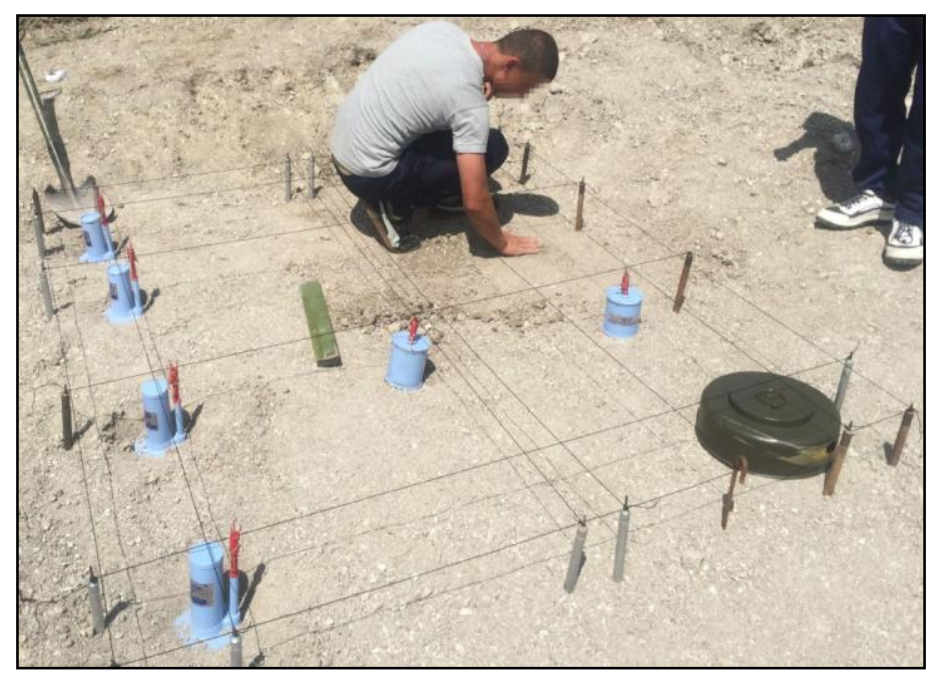

Figure 12 Placing PLs and stones in the first test field 
All PLs and stones can be seen in Figure 15 before they were buried in the minefield with the exception of two plastic PLs that stay on the surface, indicated on the figure as "Surface Mine".

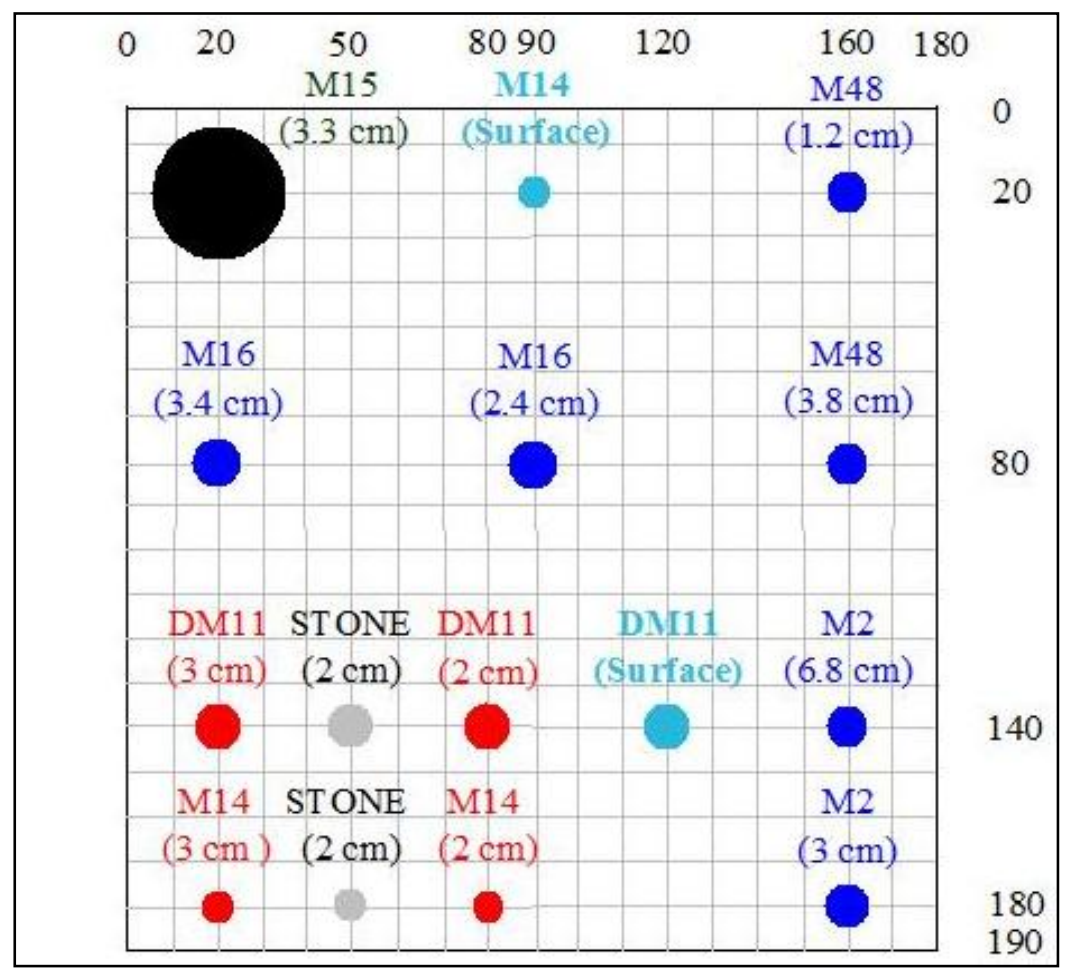

Figure 13 Layout of landmines in the test field

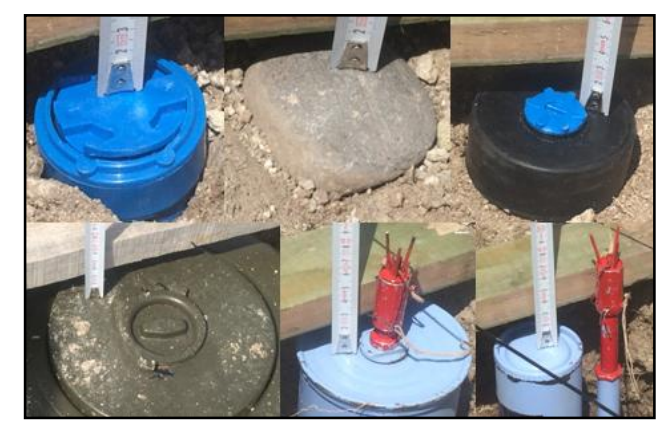

Figure 14 The depths of the PLs and stones

The PLs were buried/placed on 7 July 2015. The minefield's appearance is given in Figure 16 after all PLs and stones were buried/placed. 


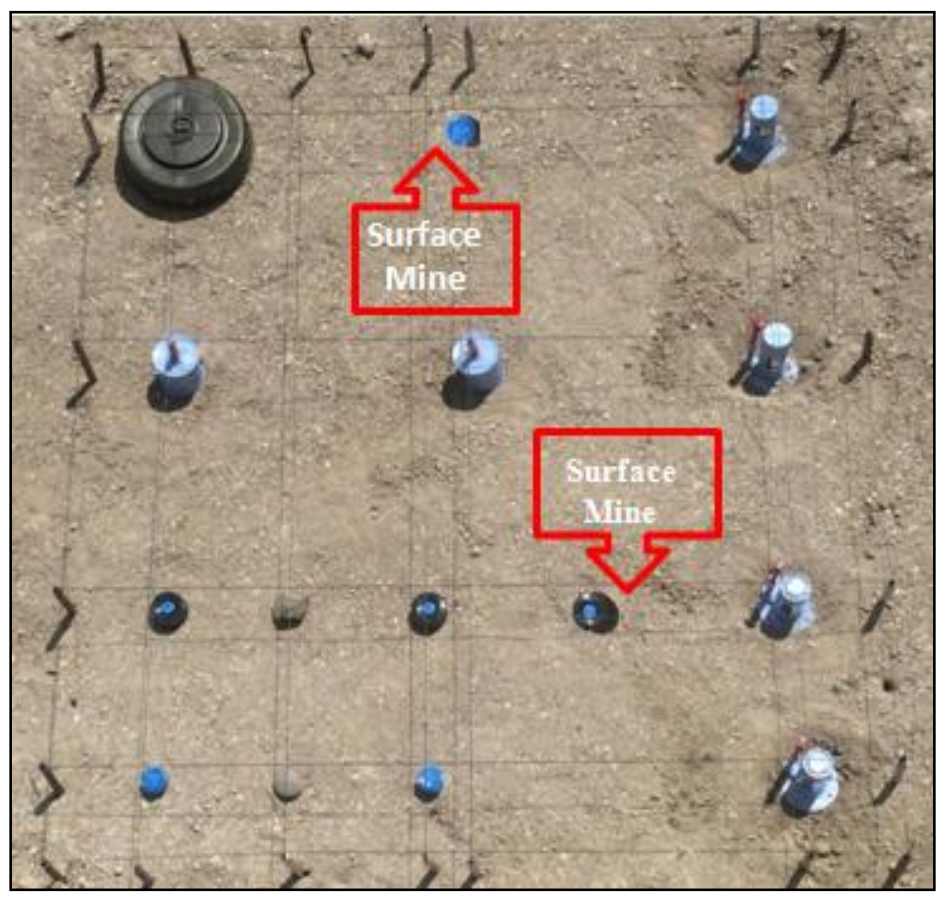

Figure 15 The PLs in the test area before burial

\subsection{Preparation of the Second Test Field}

The second test minefield was formed in order to evaluate proposed algorithm's performance in different conditions with larger number and same type of plastic antipersonnel PL, namely DM11. The different conditions are different soil type (Clay loam) and soil humidity as well as different weather and season. The second minefield's construction and placement of the PLs and the stones were carried out on 18 of February 2016 during the rainy season of Ankara.

The humid clay-loam soil surface was cleaned from sparse vegetation. Then, a $1.5 \mathrm{x}$ $1.5 \mathrm{~m}$ area was dug $20 \mathrm{~cm}$ deep and all objects were removed as done in the first minefield. The preparation of the area can be seen in Figure 17 and Figure 18.

Thirteen PLs and two stones were used in total. Eleven PLs and two stones were buried and two plastic PLs that are a DM 11 and a M14 were located on the surface of the minefield. The layout of the minefield is given in Figure 19 along with the depths that mines are buried at. 


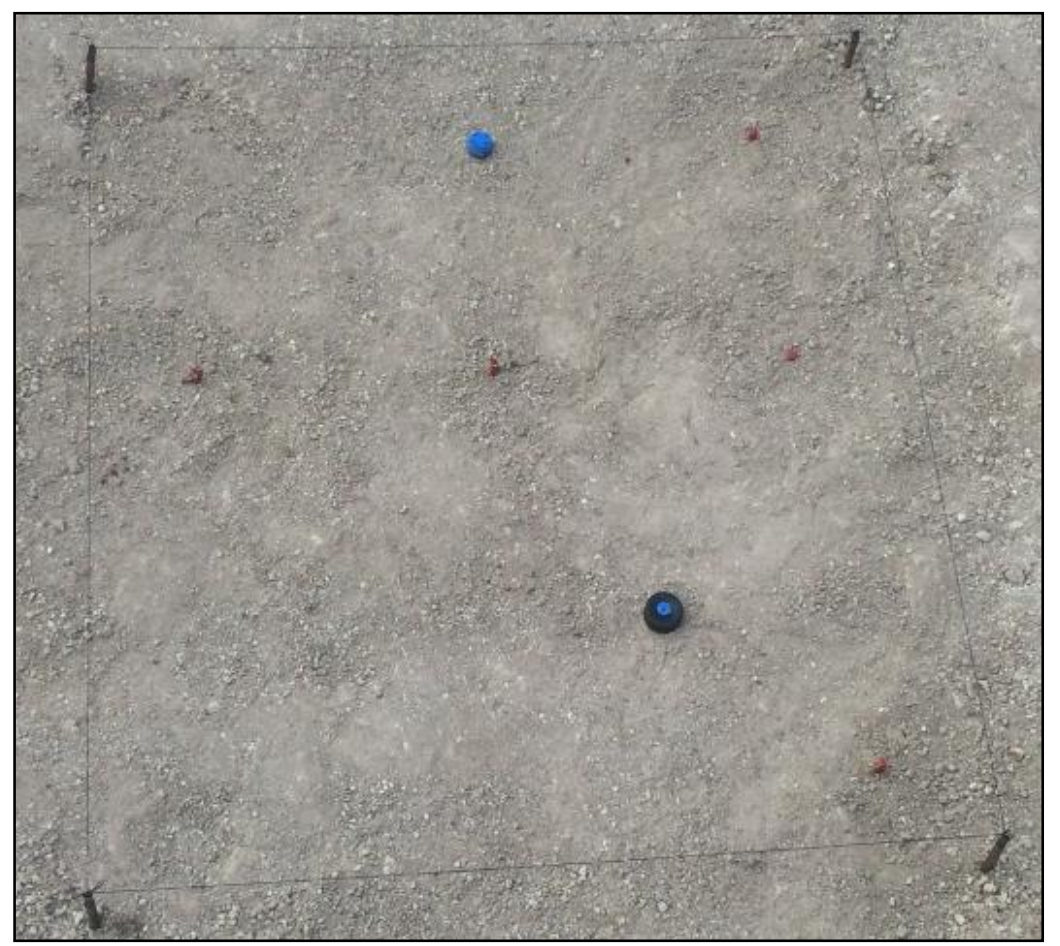

Figure 16 The final appearance of the minefield after all PLs and stones were buried/placed

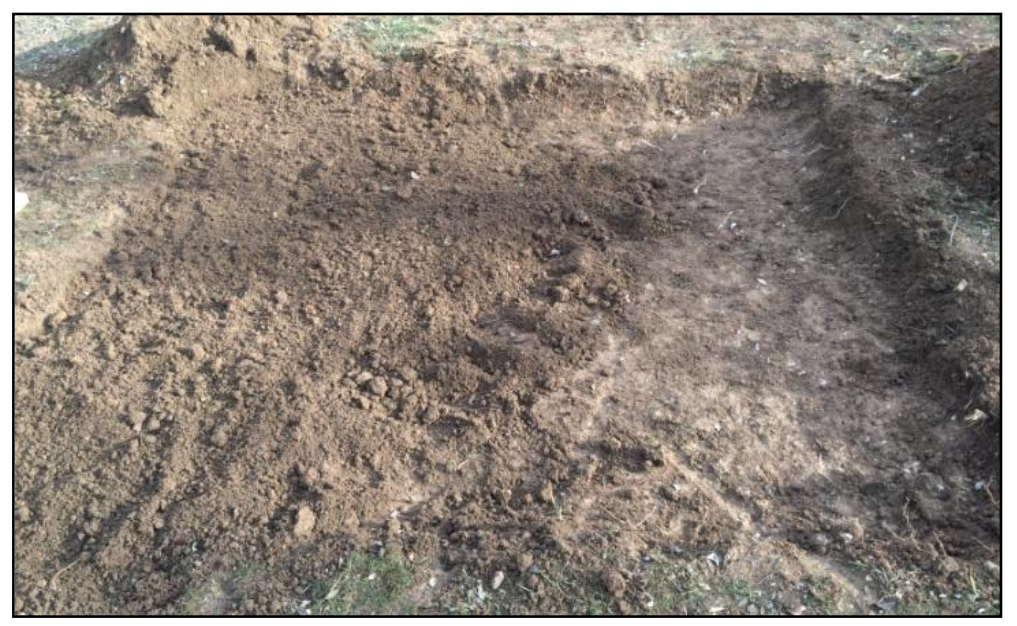

Figure 17 Preparation of the second minefield - 1 


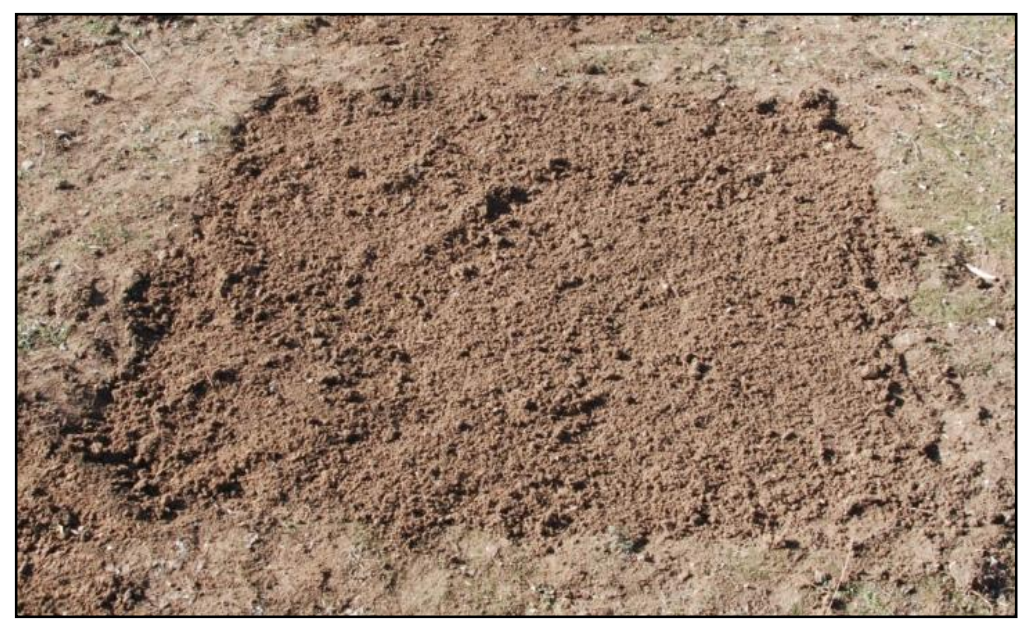

Figure 18 Preparation of the second minefield - 2

Even though DM11 landmines should be emplaced at a depth of one $\mathrm{cm}$, in real minefield they can be located at different depths and most of the times the depths are larger than one $\mathrm{cm}$. Therefore, in order to simulate real minefield conditions PLs were emplaced at depths of three and four $\mathrm{cm}$.

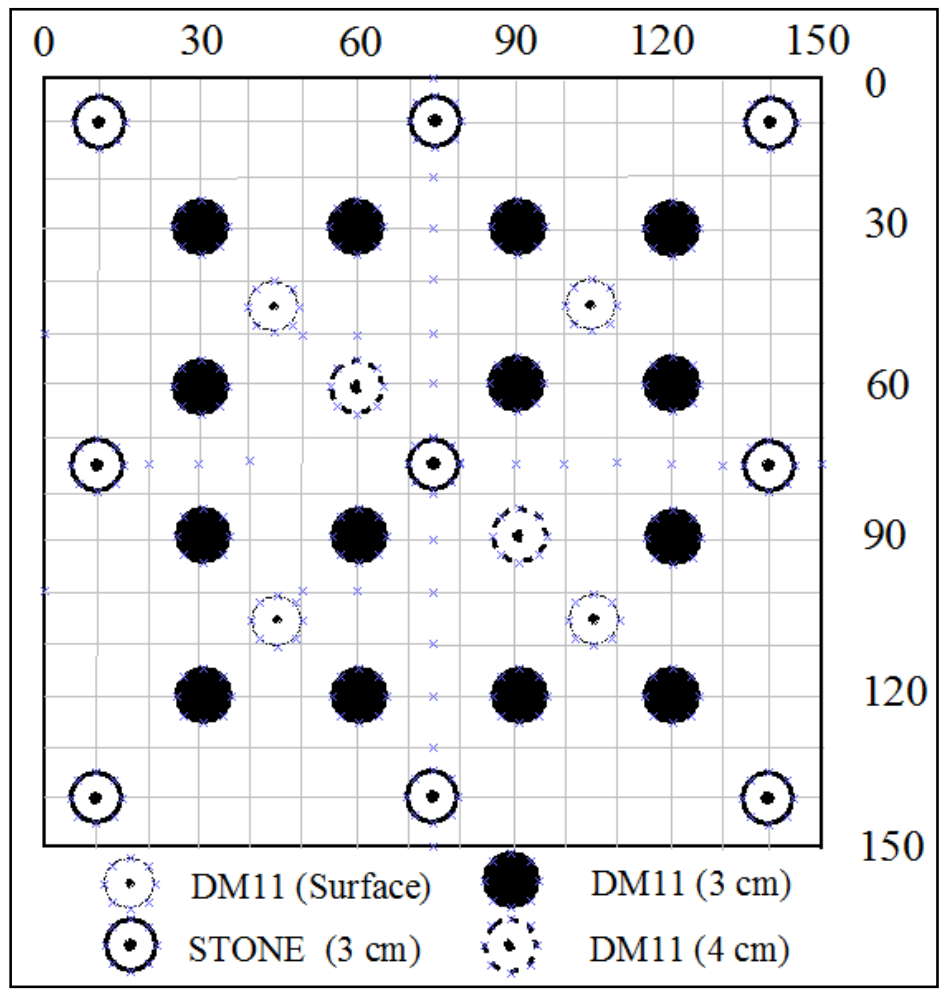

Figure 19 Layout of landmines in the second minefield 26 
Fourteen PLs were emplaced at a depth of three $\mathrm{cm}$ to evaluate the performance of the proposed method correctly. Two DM11 PLs were buried at four $\mathrm{cm}$ deep to see the performance of the method at larger depths. In addition, nine stones with shapes and diameters similar to the DM11 were emplaced at three $\mathrm{cm}$ deep and lastly four DM11 PLs were located on the soil surface.

All PLs and stones can be seen in Figure 20 before they were buried in the minefield with the exception of four plastic DM11 PLs that stay on the surface, indicated on the figure as "Surface Mine". Final form of the second minefield is given in Figure 21.

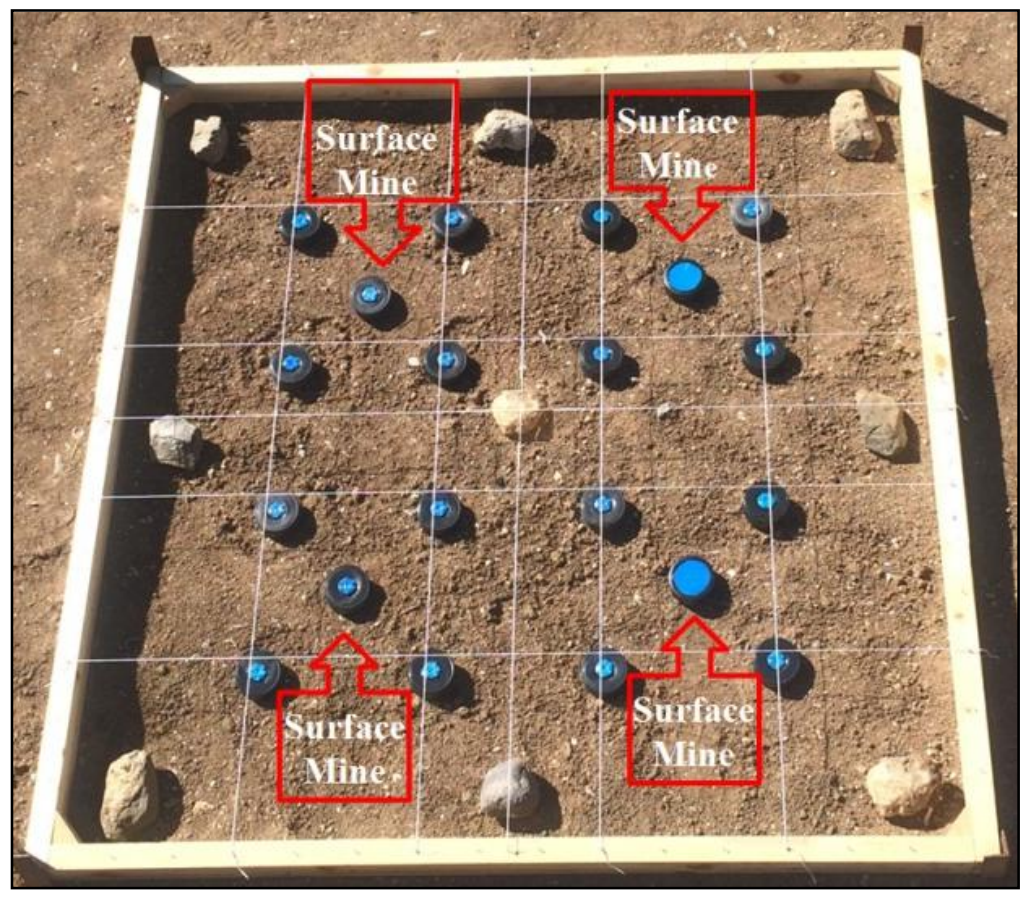

Figure 20 The PLs in the second minefield before burial 


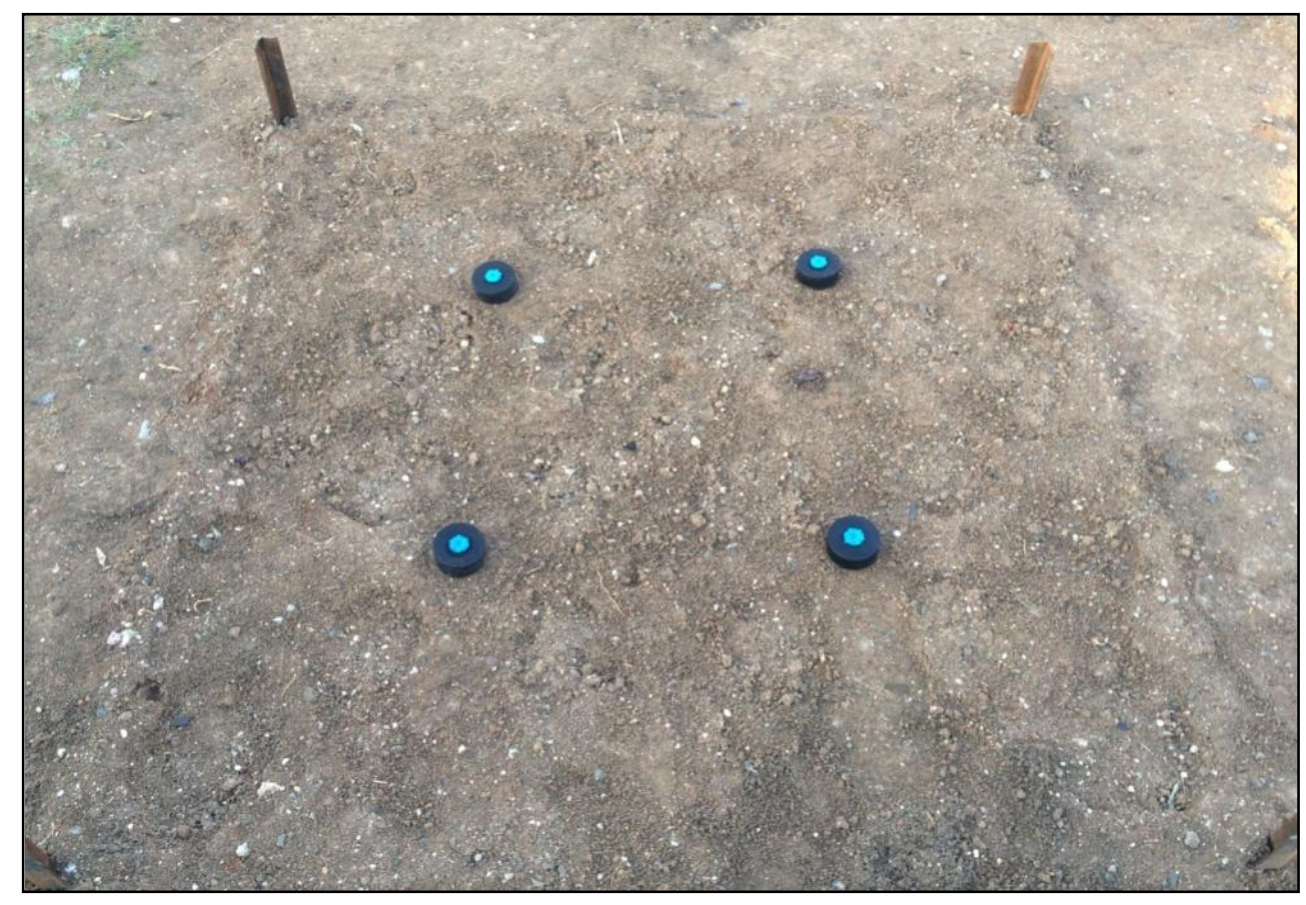

Figure 21 Final appearance of the second minefield 


\section{CHAPTER 4}

\section{DATA ACQUISITION}

In this section, the data used in this study is presented. The details of how the images are acquired are described in the following sections.

\subsection{Cameras and Their Characteristics}

FLIR T 650 SC and ATOM ${ }^{\mathrm{TM}} 1024$ IR cameras were used for collecting the data. The cameras' properties are given in Table 1.

Table 1 IR cameras and their properties

\begin{tabular}{|l|l|l|l|}
\hline Camera & IR detector & Array size & Spectral range \\
\hline ATOM $^{\mathrm{TM}} 1024$ & Uncooled Micro bolometer & $1024 \times 768$ & $8-14.0 \mu \mathrm{m}$ \\
\hline FLIR T650SC & Uncooled Micro bolometer & $640 \times 480$ & $7.5-13.0 \mu \mathrm{m}$ \\
\hline
\end{tabular}

\subsection{Viewing Geometry}

Two different cameras were used collecting the data from the first minefield. The look angles with respect to nadir were approximately $27^{\circ}-30^{0}$, and the elevations of cameras from the soil surface were $524 \mathrm{~cm}$ (FLIR T650SC), and $555 \mathrm{~cm}\left(\right.$ ATOM $\left.^{\mathrm{TM}} 1024\right)$.

The cameras were located on a platform that was parallel to the minefield and did not cast any shadow on the minefield during the day. In addition to that, platform's elevation enabled cameras to have a 2 × 2 m footprint. Therefore, cameras could image whole minefield. FLIR T650SC's viewing geometry is given in Figure 22, and ATOM ${ }^{\mathrm{TM}}$ 1024 's viewing geometry is given in Figure 23. 
Only FLIR T650SC camera was used for capturing data from the second minefield. In this setting, the camera's view angle was approximately $35^{\circ}$ and elevation of camera from the soil surface was $365 \mathrm{~cm}$.

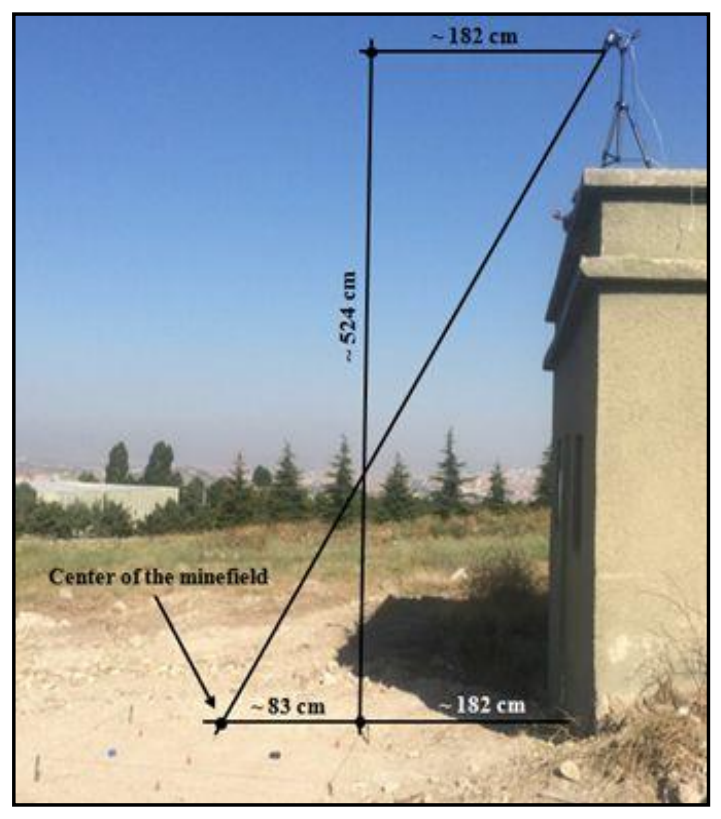

Figure 22 FLIR T 650 SC's viewing geometry

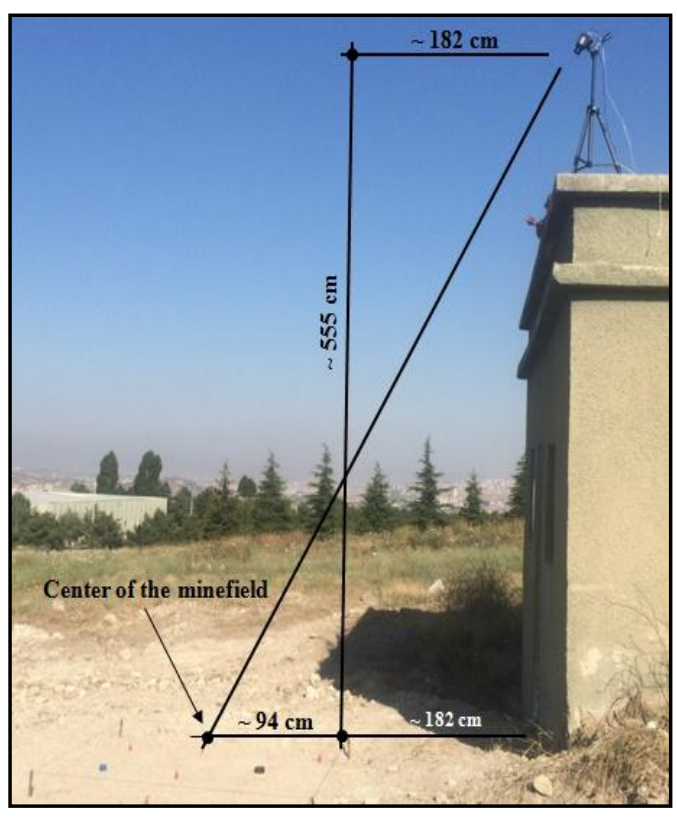

Figure $23 \mathrm{ATOM}^{\mathrm{TM}} 1024$ 's viewing geometry 


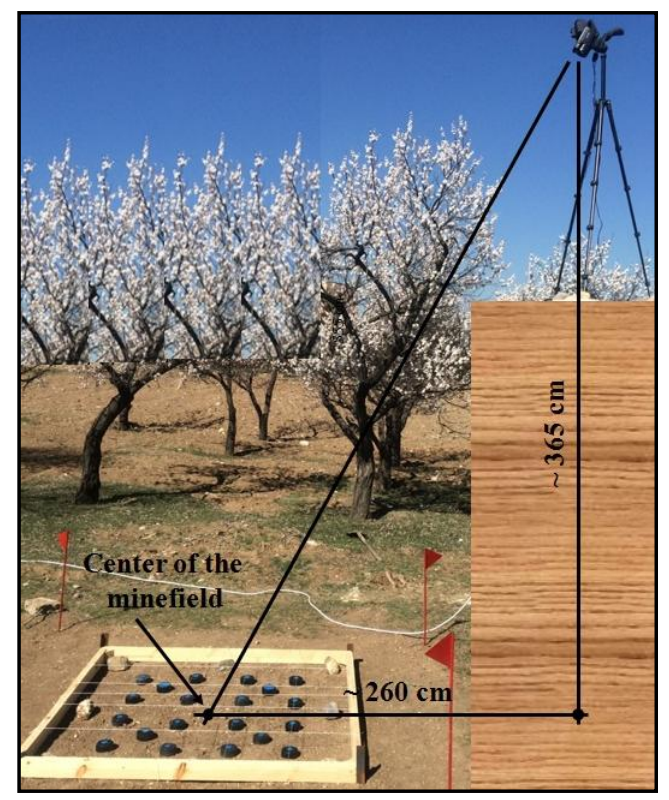

Figure 24 The second minefield camera viewing geometry

\subsection{First Acquisition: One Day After Establishment of the First Minefield}

The first data set was captured on 8 July 2015, one day after PLs and stones were emplaced in the first minefield. As mentioned in section 2.6, the landmines can be thought of as an unnatural volume for flow of the heat inside the soil. This will surely create a specific spatiotemporal thermal pattern on the surface of the soil. If the anomalies occur because of disturbed soil, it is called the surface effect [21]. The surface effect is detectable for only short period after landmine planting. During this period, the thermal contrast is quite noticeable [20]. Therefore, if surface effect can give detectible spatiotemporal thermal pattern that is detectable by IR sensors on the surface where there are newly buried objects, IR sensor can be used for finding improvised explosive devices that are one of the biggest threats for civilian and military personnel all over the word. Examples of images that were captured from 8 July 2015 at 11:10 a.m. to 9 July 2015 at 05:10 are given in Figure 25 and Figure 26. These two images were taken by ATOM $^{\mathrm{TM}}$ 1024. The 768 x 1024-pixel camera's ground footprint was approximately $2 \mathrm{~m} \times 2 \mathrm{~m}$ from the highest suitable platform, which corresponds to approximately 0.25 $\mathrm{x} 0.25 \mathrm{~cm}$ of resolution. 


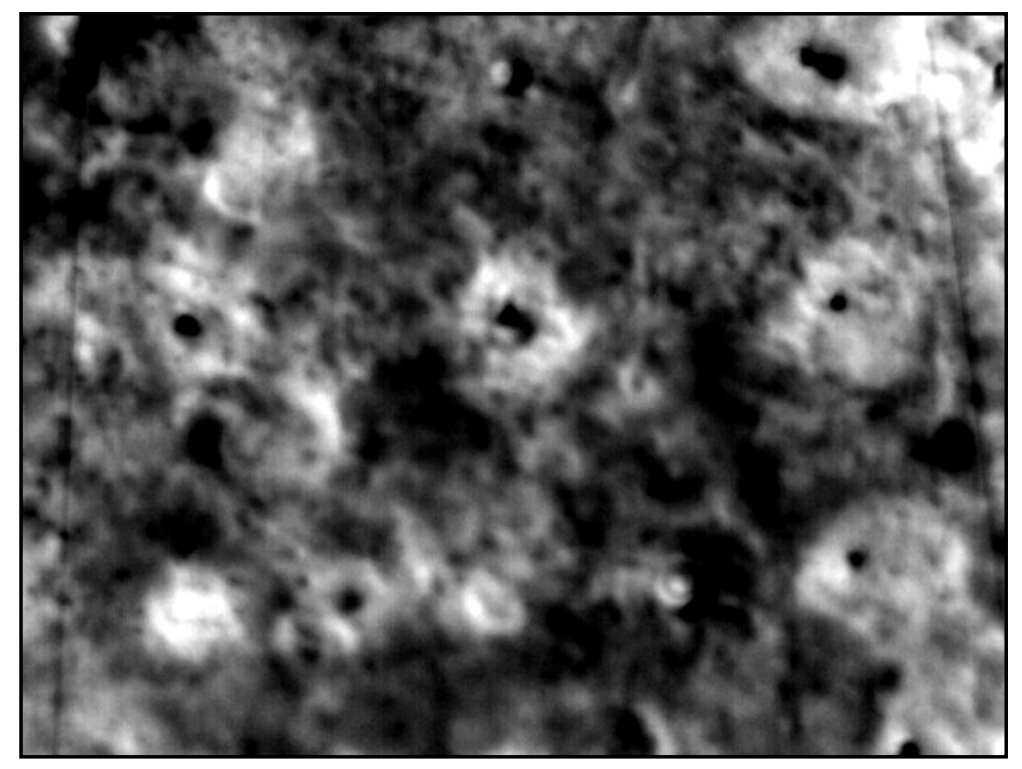

Figure 25 The image captured on 8th of July 2015 at 11:10 a.m. by ATOM $^{\mathrm{TM}} 1024$

The time series consists of images that are captured with 30 minutes time intervals. At every 30 minutes time interval, four images are captured in less than one second.

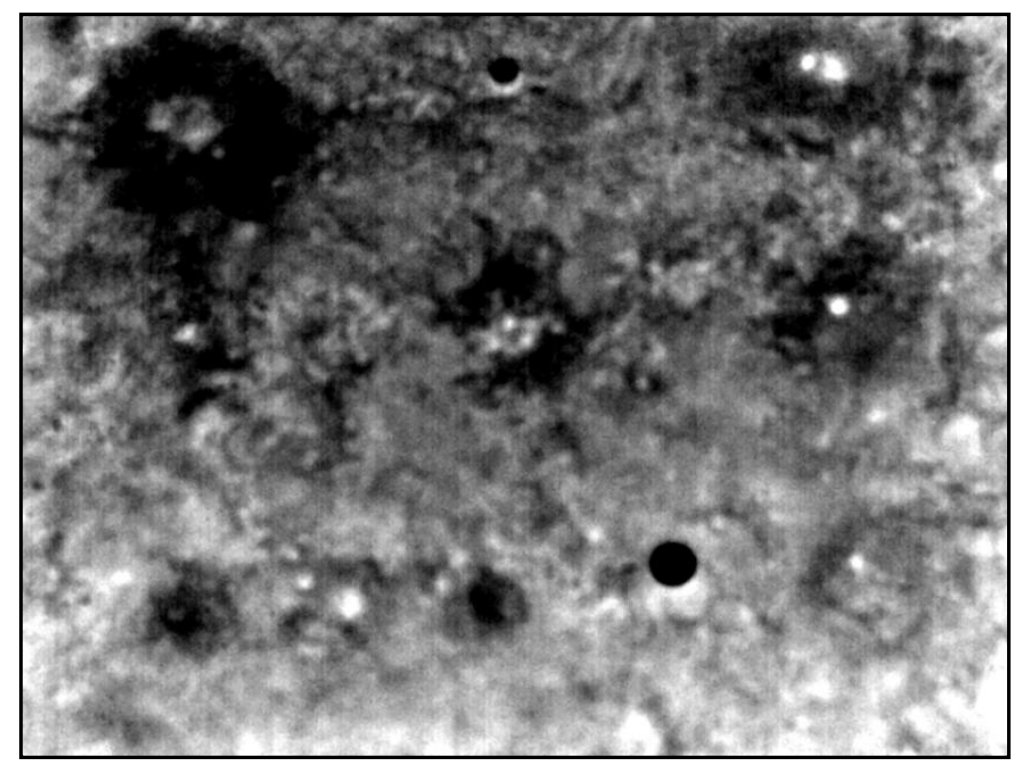

Figure 26 The image captured on ninth of July 2015 at 05:10 a.m.

$$
\text { by } \mathrm{ATOM}^{\mathrm{TM}} 1024
$$




\subsection{Second Acquisition: Two Months After the Establishment of the First Minefield}

The FLIR T 650 SC and ATOM ${ }^{\mathrm{TM}} 1024$ IR cameras were used together for collecting the data during the second acquisition. The data were captured on 8-9th of September 2015 two months after establishment of the first minefield.

ATOM $^{\text {TM }} 1024$ captured diurnal cycle data with 15 minutes interval. FLIR T650SC started to collect data at 21.12 o'clock on 8 September 2015 and finished at 13.27 o'clock on 9 September 2015 with 15 minutes interval as well. Every 15 minutes time interval, cameras captured four images within one second. The 480 x 640 pixel FLIR T650SC's ground footprint was approximately $2 \mathrm{~m} \times 2 \mathrm{~m}$ from the platform, which corresponds to approximately $0.45 \times 0.45 \mathrm{~cm}$ of resolution. Besides capturing images, environment temperature was recorded during diurnal cycle. One shut of the image, which was taken at 2 a.m. by FLIR T650SC, is shown in Figure 27 as an example.

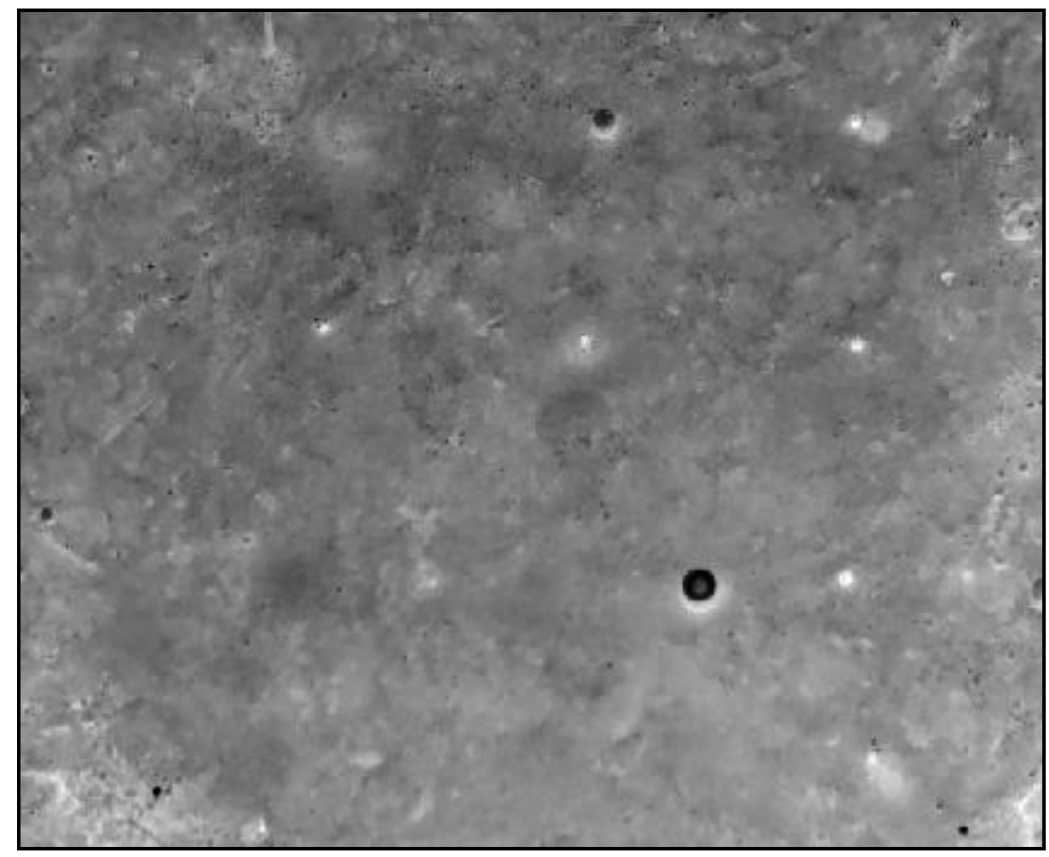

Figure 27 The image captured at 2:00 a.m. by FLIR T 650 SC 


\subsection{Third Acquisition from the Second Minefield}

The FLIR T 650 camera was used for collecting the data. The data was captured on 9-10 March of 2016, 20 days after establishment of the second minefield. The imaging was started at 12:00 pm and finished next day at 11:45 am, every 15 minutes time interval, the camera captured four images within one second.

The 480 x 640 pixel FLIR T650SC's ground footprint was approximately $1.8 \mathrm{~m}$ x $1.8 \mathrm{~m}$ from the platform, which corresponds to approximately 0.34 x $0.34 \mathrm{~cm}$ of resolution. One shot of the image, which was taken at 6:45 a.m. can be seen in Figure 28 as an example.

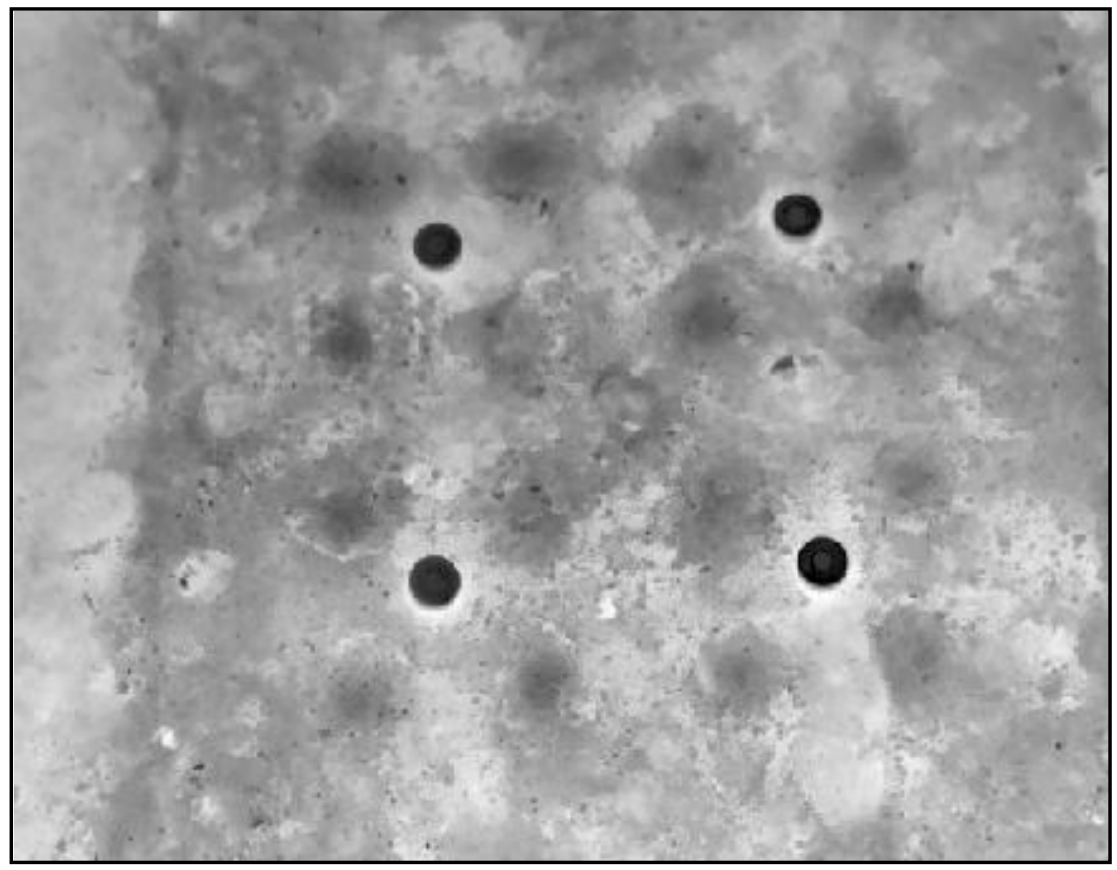

Figure 28 The image was captured at 6:45 a.m. 


\section{CHAPTER 5}

\section{SIMULATION OF HEAT FLOW IN MINEFIELDS}

The aim of the simulation is to enhance proposed method through detailed and rigorous analyses of heat transfer in minefields with various parameters (time of the day, soil type, landmine type, burial depth of mine etc.). The weather data and soil thermal properties that were used during simulations correspond to the two test areas and test dates. The soil and PLs are assumed homogeneous. Software-based finite element model (FEM) was used to simulate thermal behavior of buried land mines in the soil.

\subsection{Simulation Software}

All simulations were performed using MATLAB $^{\circledR}$ 2015a and the Partial Differential Equation Toolbox using a PC with HP Intel (R) Core (TM) i5-3210M CPU @ 2.50GHz, 8.00 GB RAM and 64-bit operating system. Finite elements method was used for simulations.

\subsection{Assumptions and Parameters}

Since the ideal geometry is circularly symmetric, it is reasonable to assume that the temperature pattern is circularly symmetric as well and hence a 2-D simulation in a vertical plane passing through the center of the mine is sufficient. The soil and the PLs are assumed homogeneous and isotropic, and soil surface is assumed smooth in this study. In addition, the soil is assumed dry for the first test area and slightly moist for the second test area. 


\subsubsection{Boundary and Initial Conditions}

The heat transfer function (4) needs initial and boundary conditions. The upper boundary, which is the soil surface, is affected continuously from thermal conditions. Therefore, heat flux, which is given in next section, is time variant. The simulation takes upper boundary condition from a time variant heat flux model. Along the vertical boundaries, the normal fluxes are set the zero [37], and it is assumed that there is heat balance along the vertical boundaries [32] along which temperature gradients are set to zero.

In order to find proper depth of the soil, the bottom edge is assumed to have a zero heat flow boundary (isolated), that is, specific form of Neumann boundary condition in first trials. After that, the depth of the simulation was changed until finding the proper depth, which is given by negligible temperature change during diurnal cycle along the bottom boundary of the simulation (soil). The maximum temperature change along the bottom became $0.0055^{\circ} \mathrm{K}$ peak-to-peak when the depth was set to two meters, and the average temperature was 291.8 K (Figure 29). Fixing bottom boundary as isolated does not simulate the real condition. Therefore, the bottom boundary is assumed to have Dirichlet boundary and it is set to the constant value of $291.8 \mathrm{~K}$.

The initial air temperature is set to $293 \mathrm{~K}$ and bottom nodes' initial temperature is set to 291.8 K. The test areas' geographic information and thermal values are given in Table 2.

Table 2 Test areas' geographic and thermal properties

\begin{tabular}{|l|c|c|c|c|c|}
\hline $\begin{array}{l}\text { Test } \\
\text { Area }\end{array}$ & $\begin{array}{c}\text { Latitude } \\
(\text { degree) }\end{array}$ & $\begin{array}{c}\text { Longitude } \\
(\text { degree })\end{array}$ & $\begin{array}{c}\text { Altitude } \\
(\mathrm{km})\end{array}$ & $\begin{array}{c}\text { Minimum } \\
\text { Temperature } \\
(\mathrm{K})\end{array}$ & $\begin{array}{c}\text { Maximum } \\
\text { Temperature } \\
(\mathrm{K})\end{array}$ \\
\hline First & 39.9403 & 32.9171 & 1.033 & 284 & 309 \\
\hline Second & 39.9092 & 32.8413 & 0.926 & 273 & 292 \\
\hline
\end{tabular}




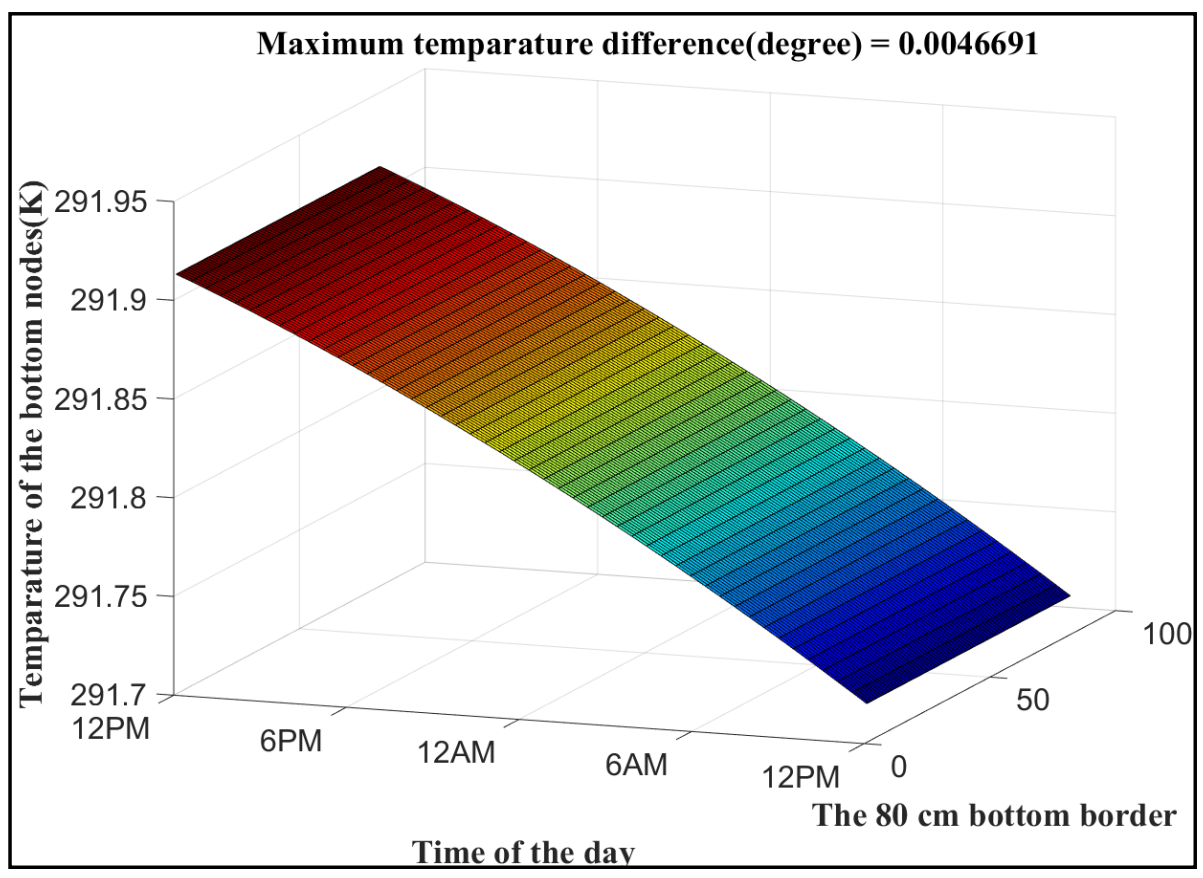

Figure 29 Bottom border temperature change when the soil depth is one meter Soil and PLs' Properties

M48 metal and DM11 plastic PLs are used in the simulation. PLs' dimensions and thermal properties [9], [38] are given in Table 3.

Table 3 PLs' dimensions and assumed thermal properties

\begin{tabular}{|l|c|c|c|c|c|}
\hline PL & $\begin{array}{c}\text { Height } \\
(\mathrm{mm})\end{array}$ & $\begin{array}{c}\text { Diameter } \\
(\mathrm{mm})\end{array}$ & $\begin{array}{c}\text { Thermal } \\
\text { Conductivity } \\
(\mathrm{W} / \mathrm{m}-\mathrm{K})\end{array}$ & $\begin{array}{c}\text { Specific } \\
\text { Heat } \\
(\mathrm{J} / \mathrm{kg}-\mathrm{ge}\end{array}$ & $\begin{array}{c}\text { Density } \\
\left(\mathrm{kg} / \mathrm{m}^{3}\right)\end{array}$ \\
\hline M48 PL & 165 & 63.5 & 54 & 465 & 7830 \\
\hline DM11 PL & 37 & 81 & 0.5 & 1260 & 1760 \\
\hline
\end{tabular}

Soils' thermal properties and densities [38], [39] are given in Table 4.

Table 4 Soils' assumed thermal properties and densities

\begin{tabular}{|l|c|c|c|}
\hline Soil Type & $\begin{array}{c}\text { Thermal } \\
\text { Conductivity } \\
(\mathrm{W} / \mathrm{m}-\mathrm{K})\end{array}$ & $\begin{array}{c}\text { Specific } \\
\text { Heat } \\
(\mathrm{J} / \mathrm{kg}-\mathrm{K})\end{array}$ & $\begin{array}{c}\text { Density } \\
\left(\mathrm{kg} / \mathrm{m}^{3}\right)\end{array}$ \\
\hline Clay & 2.7 & 1100 & 1900 \\
\hline Moist Clay & 3.8 & 1100 & 1900 \\
\hline
\end{tabular}




\subsection{The Mathematical Model}

\subsubsection{Physical Description of the Processes}

Heat transfer mechanism, which occurs from the soil surface to the mine, is given as follows. Convection and irradiative mechanism take place at the soil-air interface and in the soil and the buried objects interfaces conduction occurs [31]. The total heat transfer mechanism is depicted in Figure 30.

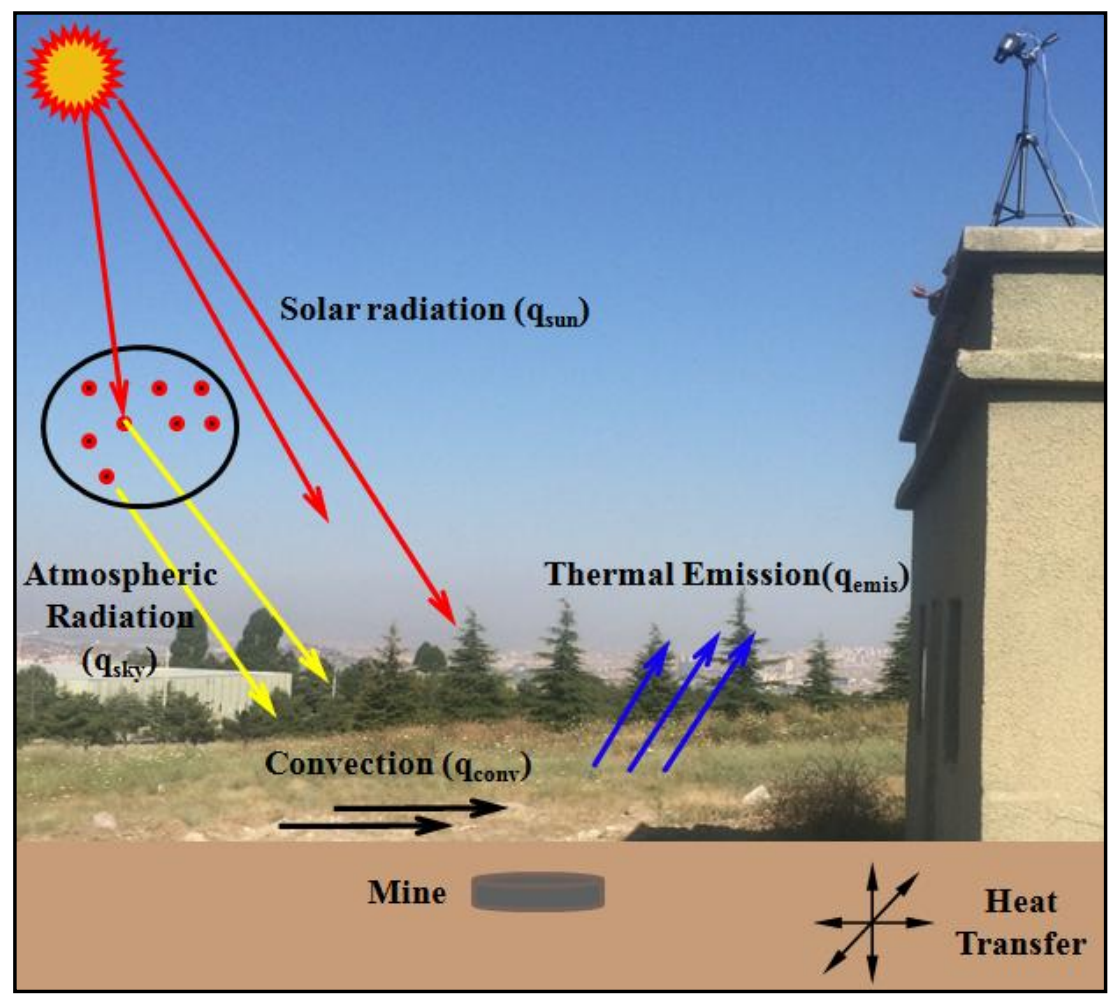

Figure 30 Heat transfer processes taking place at the air-soil interface and in the soil.

The temperature obeys the heat-flow equation [9], [31]

$\rho_{\text {soil }} c_{\text {soil }} \frac{\partial T_{\text {soil }}}{\partial t}-\nabla \cdot\left(k_{\text {soil }} \nabla T_{(\text {soil }, t)}\right)=q_{\text {net }}$ 
where $\mathrm{T}(\mathrm{K})$ is the temperature distribution, $\mathrm{k}(\mathrm{W} / \mathrm{Km})$ is the thermal conductivity, $\mathrm{c}$ $(\mathrm{J} / \mathrm{kgK})$ is the specific heat, and $\rho\left(\mathrm{kg} / \mathrm{m}^{3}\right)$ is the density of the soil. The process is time varying during the diurnal cycle, and the starting times of simulation were taken as real data captured time in this study. However, the simulation is first repeated for a few days periodically as the burn-in period.

\subsubsection{Soil-Surface Heat Flux}

The approximate net heat flux into the soil surface is given as [9]

$$
q_{\text {net }}=q_{\text {conv }}+q_{\text {sun }}+q_{\text {sky }}-q_{\text {emis }}-q_{\text {evap }}
$$

where $\boldsymbol{q}_{c o n v}\left(\mathrm{~W} / \mathrm{Km}^{2}\right)$ is the convective heat transfer at the soil-air interface. It is usually estimated as

$$
q_{\text {conv }}=h\left(T_{\text {air }}-T_{\text {surface }}\right),
$$

sometimes named Newton's law of cooling [40], where $\mathrm{h}$ is the convective heat transfer coefficient and it is accepted as $5 \mathrm{~W} / \mathrm{Km}^{2}$ based on $2 \mathrm{~m} / \mathrm{s}$ average wind speed [9].

$T_{\text {air }}$ is the time variant air temperature for diurnal cycle and is estimated as

$$
T_{\text {air }}=T_{\text {avg }}+T_{\text {amp }} \cos \left(2 \Pi\left(t_{\text {local }}-\left(12+t_{\text {coldest }}\right)\right)\right.
$$

where $T_{\text {avg }}(\mathrm{K})$ is the average temperature of the simulated day, $T_{\text {amp }}(\mathrm{K})$ is the half of the differences between maximum and minimum temperature of simulated day, $t_{\text {local }}$ is the time of the day in hours, and $t_{\text {coldest }}$ is the coldest time of simulated day in hours. $T_{\text {surface }}(\mathrm{K})$ is the soil surface temperature and it is calculated iteratively by the simulation itself.

Electromagnetic radiation is emitted from everything that has a non-zero temperature. The sun emits its energy in dominantly shorter wavelengths according to the Planck's law. Even though the Earth is cooler than the Sun, it still emits energy but with much longer wavelengths [41]. 
$\boldsymbol{q}_{\text {sun }}\left(\mathrm{W} / \mathrm{Km}^{2}\right)$ is the incident solar irradiance and it is reduced by the cloud cover, atmospheric absorption and the sun's incident cosine angle of zenith. The shortwavelength incident solar radiation model is given [9] as

$$
q_{\text {sun }}=\left(1-C_{L}\right) S_{o}(1-C) M(\phi) \operatorname{Cos}(\phi)
$$

where $C_{L}$ is the cloud cover, $C$ is the ground albedo, $S_{o}$ is the solar constant, and they are taken as $0.2,0.3$ and $1385 \mathrm{~W} / \mathrm{m}^{2}$, respectively [9]. $M(\phi)$ is the atmospheric transmissivity and it is given [42] as

$$
M(\phi)=(1-0.2) \operatorname{Cos}(\phi)^{-0.5}
$$

where $\phi$ is the incident zenith angle of the sun.

$\boldsymbol{q}_{s k y}\left(\mathrm{~W} / \mathrm{Km}^{2}\right)$ is the long-wave sky irradiance and it is given [9], [32] as $q_{s k y}=\sigma \epsilon T_{s k y}^{4}$ where $\sigma=5.67 \times 10^{-8} \mathrm{~W} / \mathrm{m}^{2} \mathrm{~K}^{4}$ is the Stephan-Boltzmann constant, $\epsilon$ is the mean emissivity of the surface, and $T_{s k y}(\mathrm{~K})$ is the effective sky radiant temperature and whose formula [42] is given as $T_{\text {sky }}=0.9 T_{\text {air }}$.

$\boldsymbol{q}_{\text {emis }}\left(W / \mathrm{Km}^{2}\right)$ is the long-wave radiation emission given [9] as $q_{\text {emis }}=\sigma \epsilon T_{\text {surface }}^{4}$. Emissivity $(\epsilon)$ values are taken as 0.95 in this study.

Even though soil $\boldsymbol{q}_{\text {evap }}$, which is latent cooling of the soil, is neglected in this study, for the sake of completeness, formulation is given [43] as

$$
q_{\text {evap }}=\rho_{\text {air }} c_{\text {cpair }} C_{\text {drag }}(W+2)\left(T_{\text {air }}-T_{\text {surface }}\right)
$$

where $\rho_{\text {air }}\left(1.16 \mathrm{~kg} / \mathrm{m}^{3}\right)$ is the density of air, $c_{\text {cpair }}(1007 \mathrm{~J} / \mathrm{kg} \mathrm{K})$ is the specific heat of air, $C_{\text {drag }}(0.002)$ is the wind drag coefficient, and $W$ is the wind speed. 


\subsection{Implementation}

The width of the simulation is set to $80 \mathrm{~cm}$ and depth is set to two meters. The PLs are located along the central vertical line. The depths of the PLs are changed to see the impact of the depth on the thermal pattern at the soil surface. As mentioned in previous section, two kinds of PLs are simulated. The example geometries are given in Figure 31 and Figure 32. M2 metal PL and DM11 plastic PL are located at three $\mathrm{cm}$ depth from the soil surface in the example geometries. The numbers in the geometry represent boundaries.

The grid size of triangles is set to one $\mathrm{cm}$. The meshed example of geometry can be seen in Figure 33.

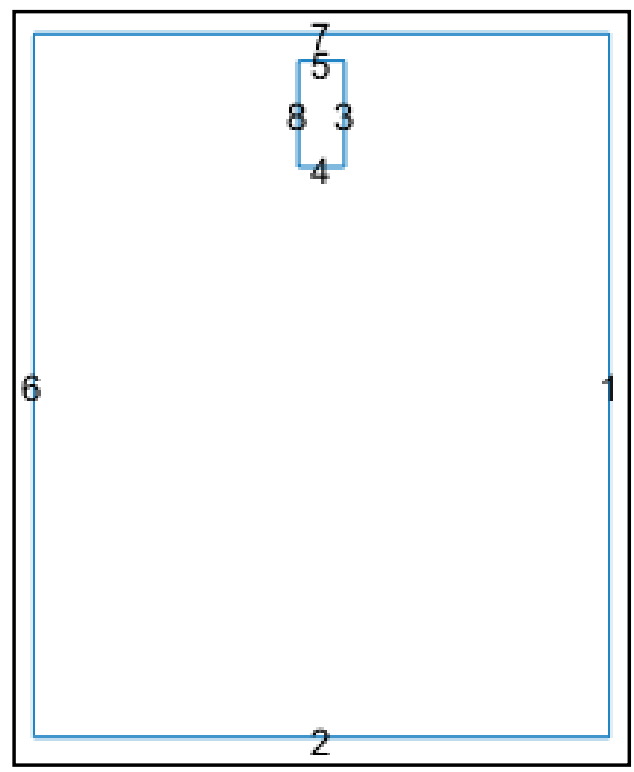

Figure 31 The simulation geometry for M2 PL metal mine 


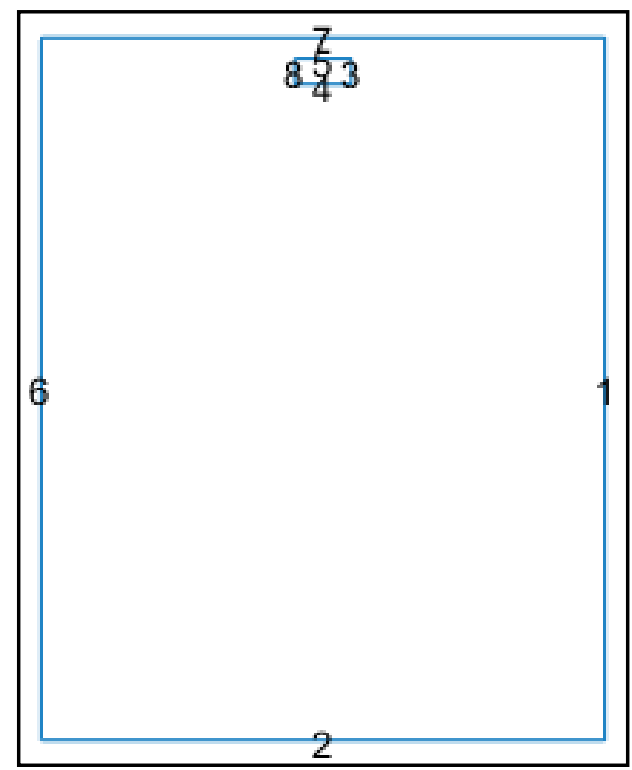

Figure 32 The simulation geometry for DM11 PL plastic mine

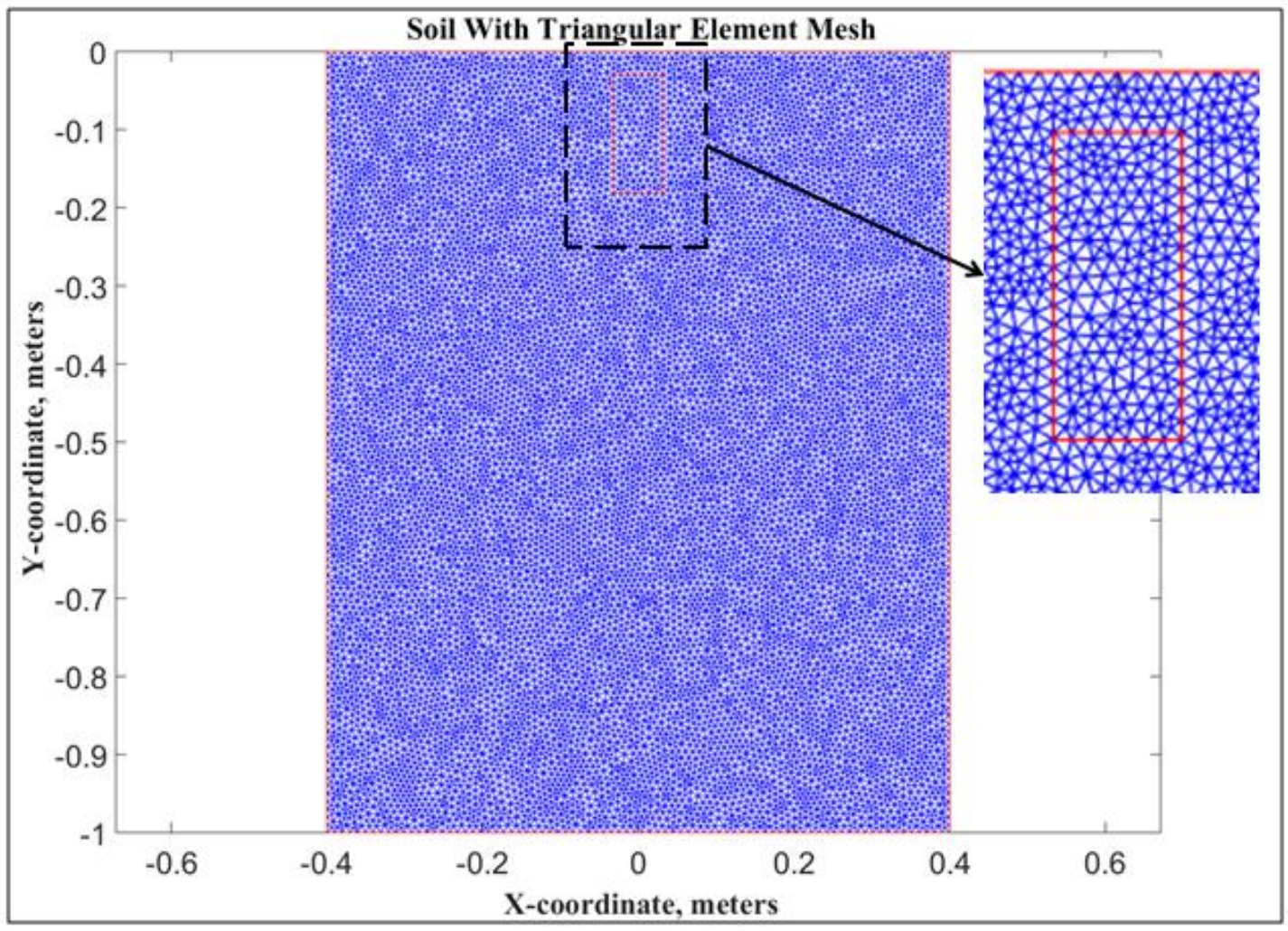

Figure 33 The meshed example of soil cross-section geometry 
The time step is set to 15 minutes, and the burn-in time is fixed to 10 days. In order to see the effect of buried PLs, temperature changes through the soil surface when the surface temperature is maximum and minimum are plotted. An example of temperature distribution through the surface is given in Figure 34 and 1-D the temperature distribution on the soil surface during entire diurnal cycle is given in Figure 35. The plastic PL is located at the center of the cross-section.

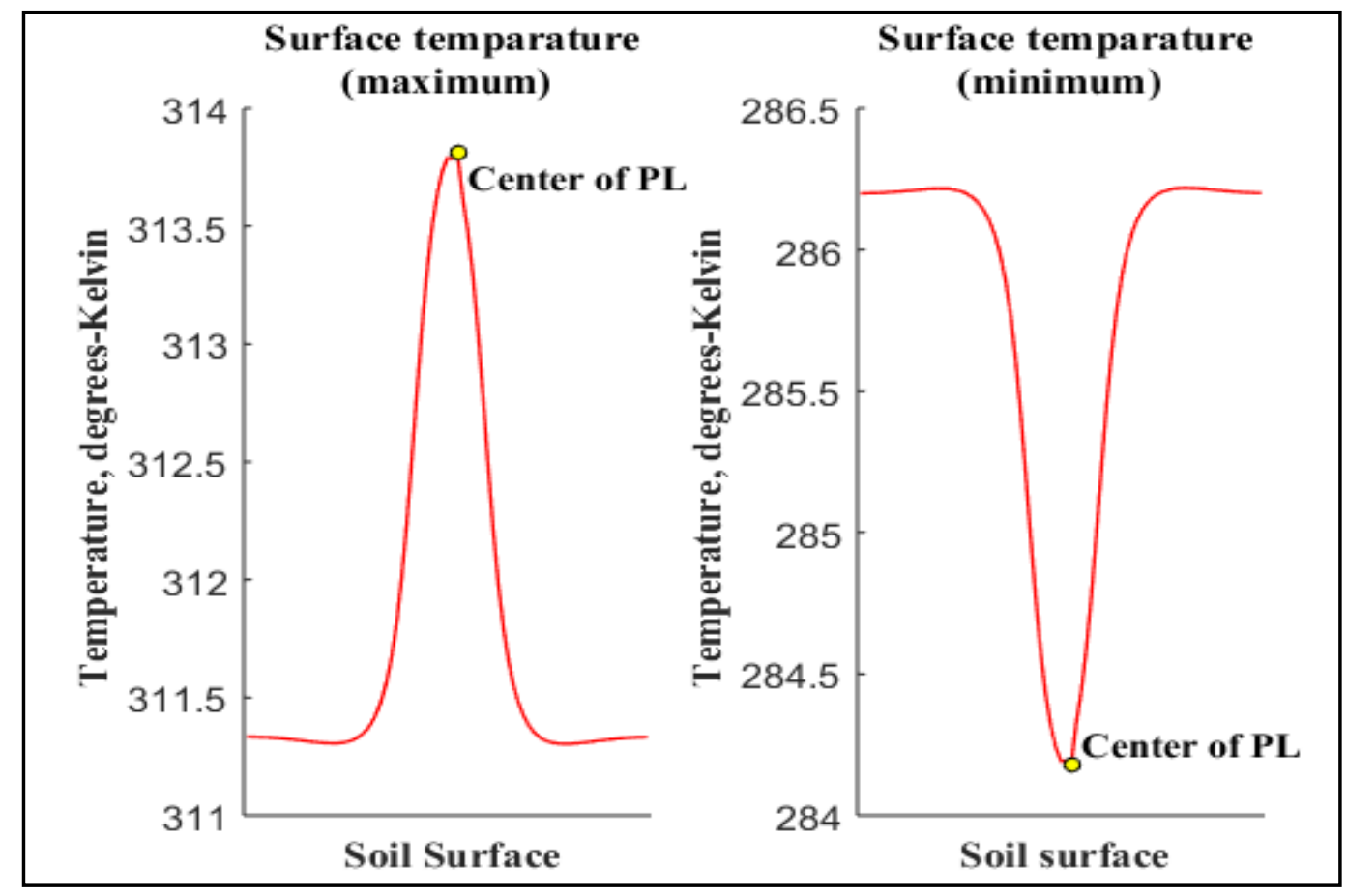

Figure 34 The soil surface' temperature distribution

An example of the temperature distribution inside the soil is also given in Figure 36 when the soil surface temperature is maximum and minimum during the diurnal cycle. 


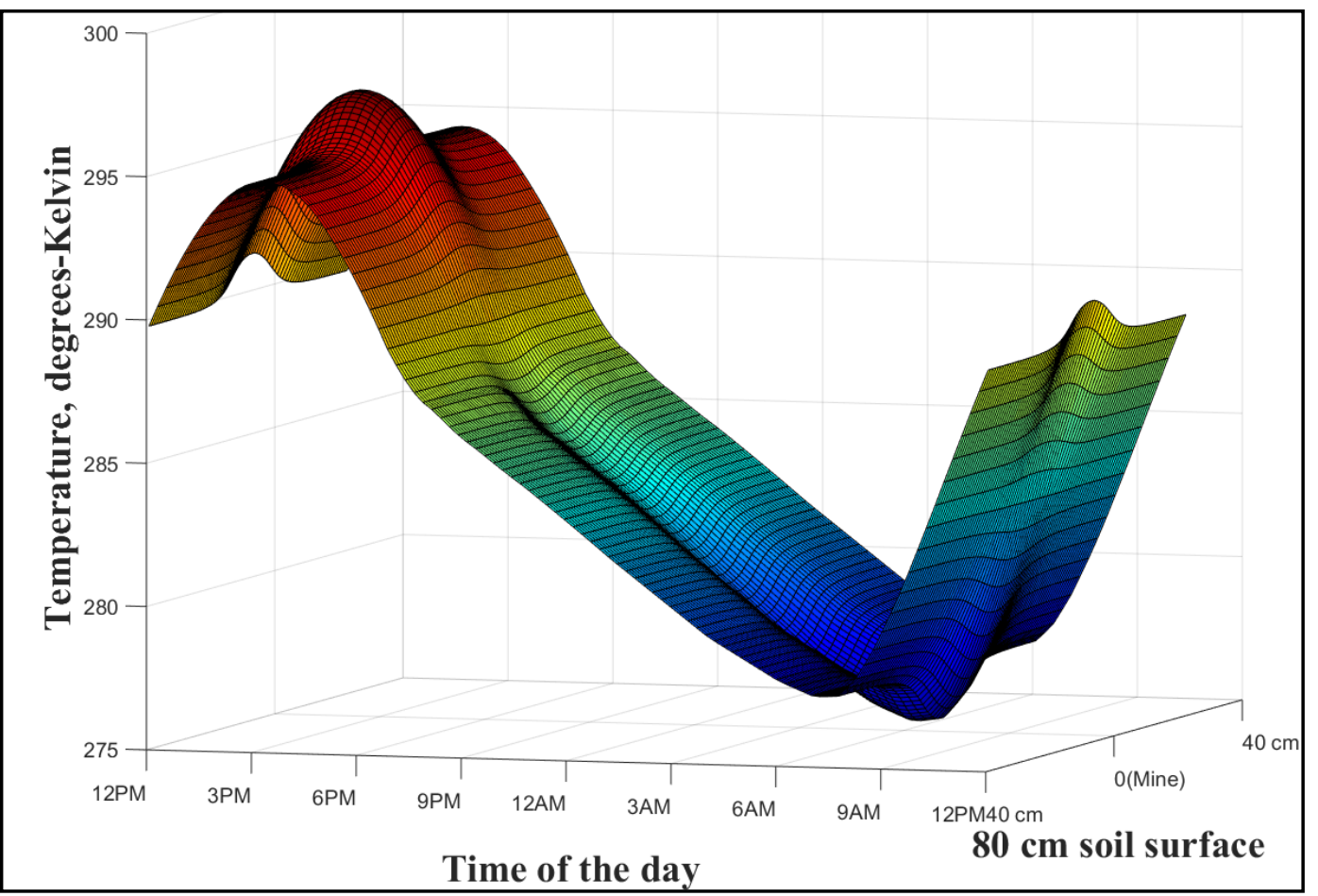

Figure 35 3-D the temperature distribution on the soil surface during the diurnal cycle

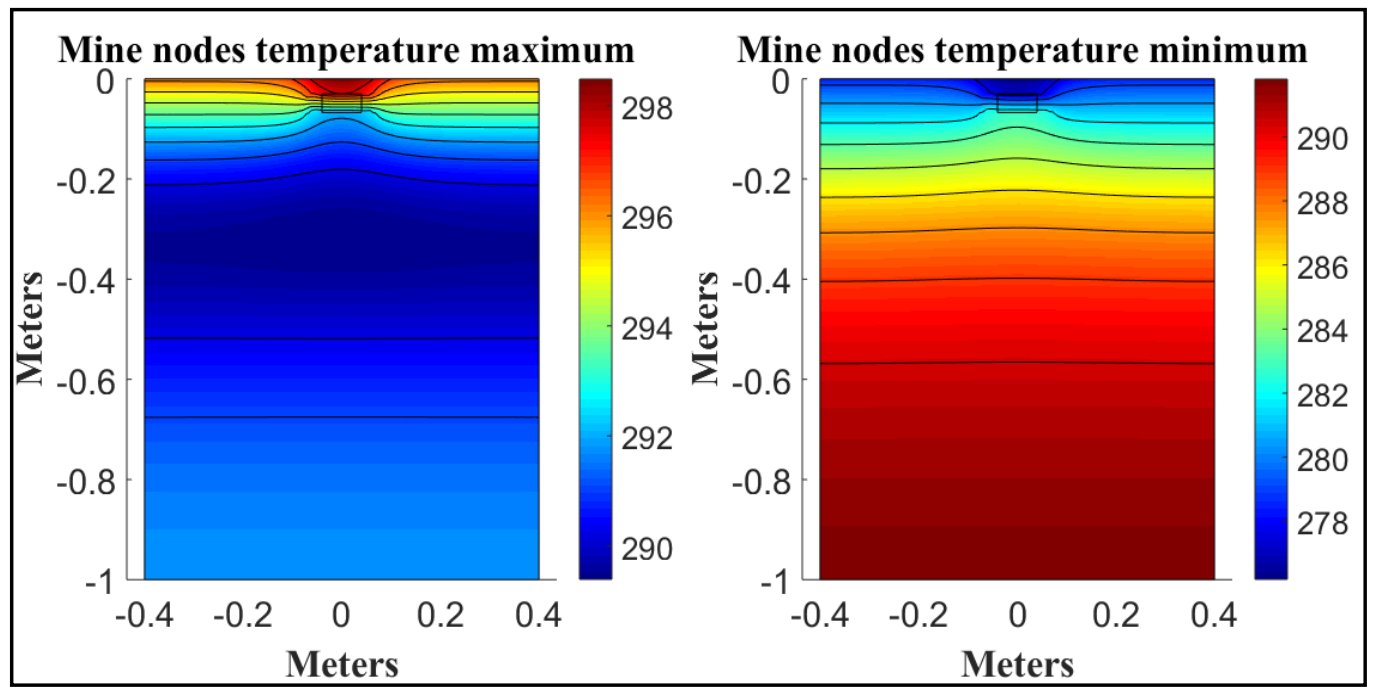

Figure 36 Temperatures distribution inside the soil 


\section{CHAPTER 6}

\section{PROPOSED LANDMINE DETECTION ALGORITHM}

The flowchart that describes the steps of the proposed algorithm is given in Figure 37. Proposed method is applied on data sets that are captured by the FLIR camera. Data Acquisition step is already given in a previous section. Noise reduction and spatial filtering are explained in detail in the preprocessing sub-sections. Dimension Reduction, Supervised Classification, and Smoothing are given in Classification sub-sections. Next, Detection is described in this section as the last step of the proposed method. All the algorithms described have been implemented in MATLAB ${ }^{\circledR}$. The results are given in the next section.

\subsection{Preprocessing}

Most of the time the soil surface temperature that encounters metal PLs and plastic PLs are different from bare soil surface temperature. However, differences are very small and can be observed during certain periods of the day. Therefore, the proposed method uses a spatial filter to detect the differences, and uses time series to recognize the temporal pattern.

\subsubsection{Noise reduction}

The time series consists of images that are captured with 15 and 30-minute time intervals. At each time step, four images are captured in less than one second and averaged to decrease the noise. The averaged images are joined together to form an 
image cube. The image cubes obtained using the ATOM and FLIR cameras are given in Table 5. Figure 38 illustrates the imaging and noise reduction process.

Even though the noise reduction process is applied on the image cubes, we have performed the whole process with un-averaged image cube and observed that results are nearly the same. We believe that the spatial filter reduces noise as a positive side effect more strongly than averaging at this phase.

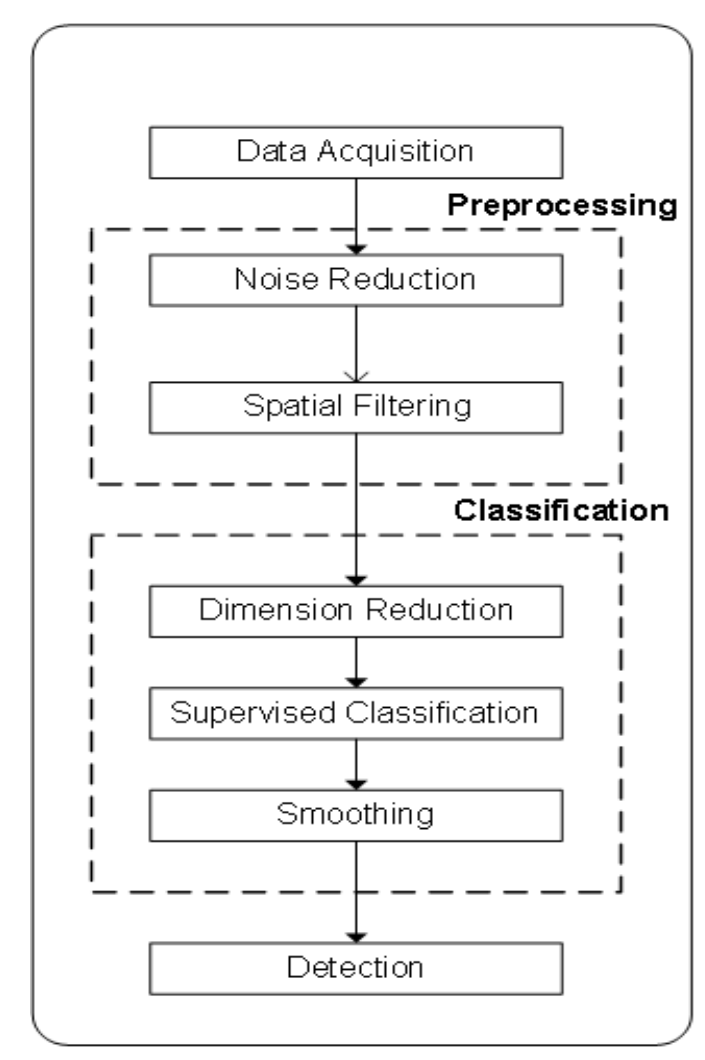

Figure 37 Steps of the proposed method

Table 5 The image cubes sizes, used cameras, and dates

\begin{tabular}{|l|c|c|c|}
\hline \multicolumn{1}{|c|}{ Captured date } & Camera & Time interval(minutes) & Cube size \\
\hline 8-9 July 2015 & ATOM $^{\mathrm{TM}}$ & 30 & $768 \times 1024 \times 48$ \\
\hline 8-9 September 2015 & ATOM $^{\mathrm{TM}}$ & 15 & $768 \times 1024 \times 96$ \\
\hline 8-9 September 2015 & FLIR & 15 & $480 \times 640 \times 39$ \\
\hline 9-10 March 2016 & FLIR & 15 & $480 \times 640 \times 96$ \\
\hline
\end{tabular}




\subsubsection{Spatial Filtering}

Depending on the size, the material and the depth of the mine, a specific spatial temperature pattern can be observed on the surface for a given environmental temperature history. A good approach can be using a matched filter for detecting these patterns. Lundberg et al. [44] have used finite element method to simulate temperature distribution on buried landmines and approximated the pattern with Gaussian functions.

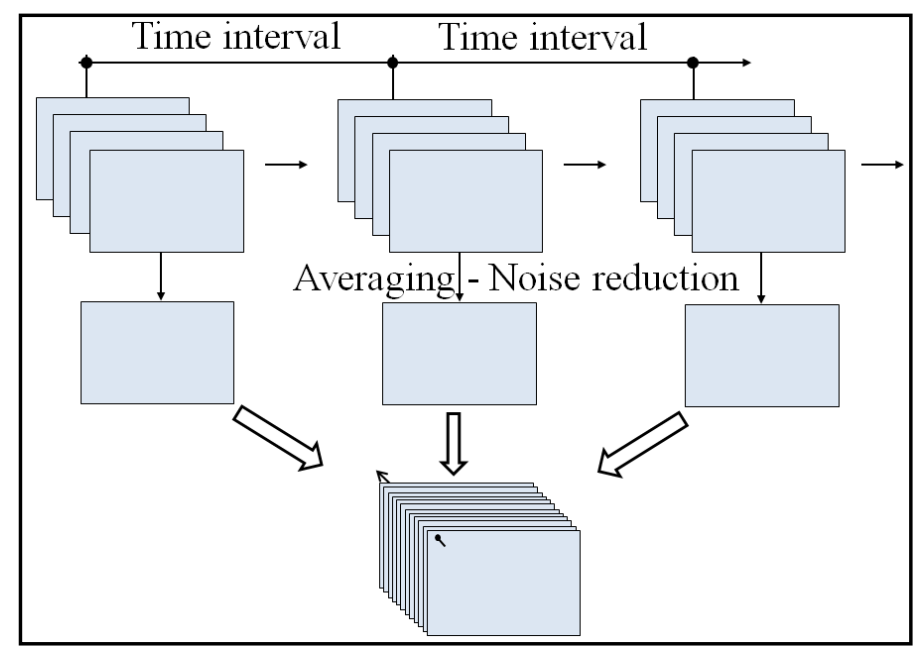

Figure 38 The image cube formation and noise removal process

For any pixel, a small and a large circle are formed having the target pixel at the center. Then the average value of the small circle is subtracted from that of the large circle. The radius sizes are selected such that for a pixel located near the center of the mine, the outer circle values are sampled from bare soil out of the mine while the inner circle values are sampled from the soil above the mine. Spatial filtered image, y, is given as

$$
y(i, j ; t)=\frac{1}{n}\left[\sum_{k \in R o} x((i, j)+k ; t)-\sum_{k \in R i} x((i, j)+k ; t)\right]
$$

where, $x$ is the original image, $R_{o}$ and $R_{i}$ are the neighborhood set of outer and inner circles respectively and $n$ is the number of elements in each of these sets. Figure 39 shows the spatial filter's circles, and sampling points. 
Example images from the filtered image cubes obtained in the second and the third acquisition (FLIR camera) are shown in Figure 40 and Figure 41, respectively. Cold spots are pronounced as dark areas and hot spots are as white areas in these images.

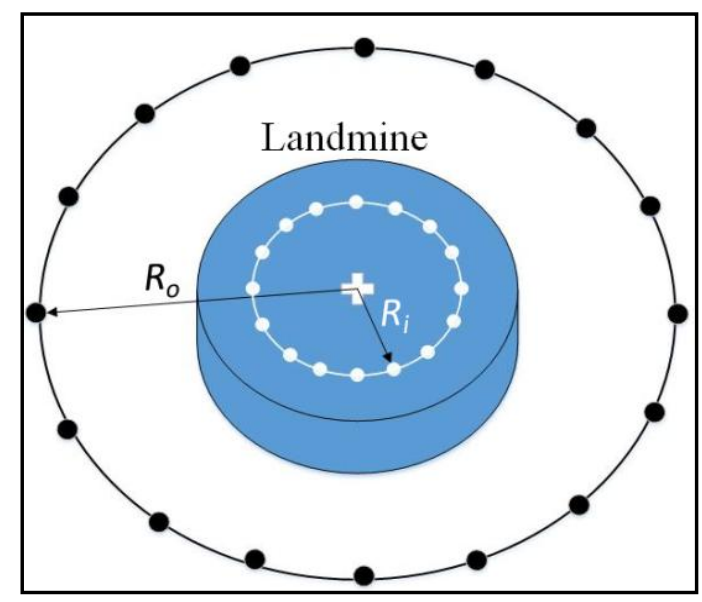

Figure 39 The circular spatial filter's representation

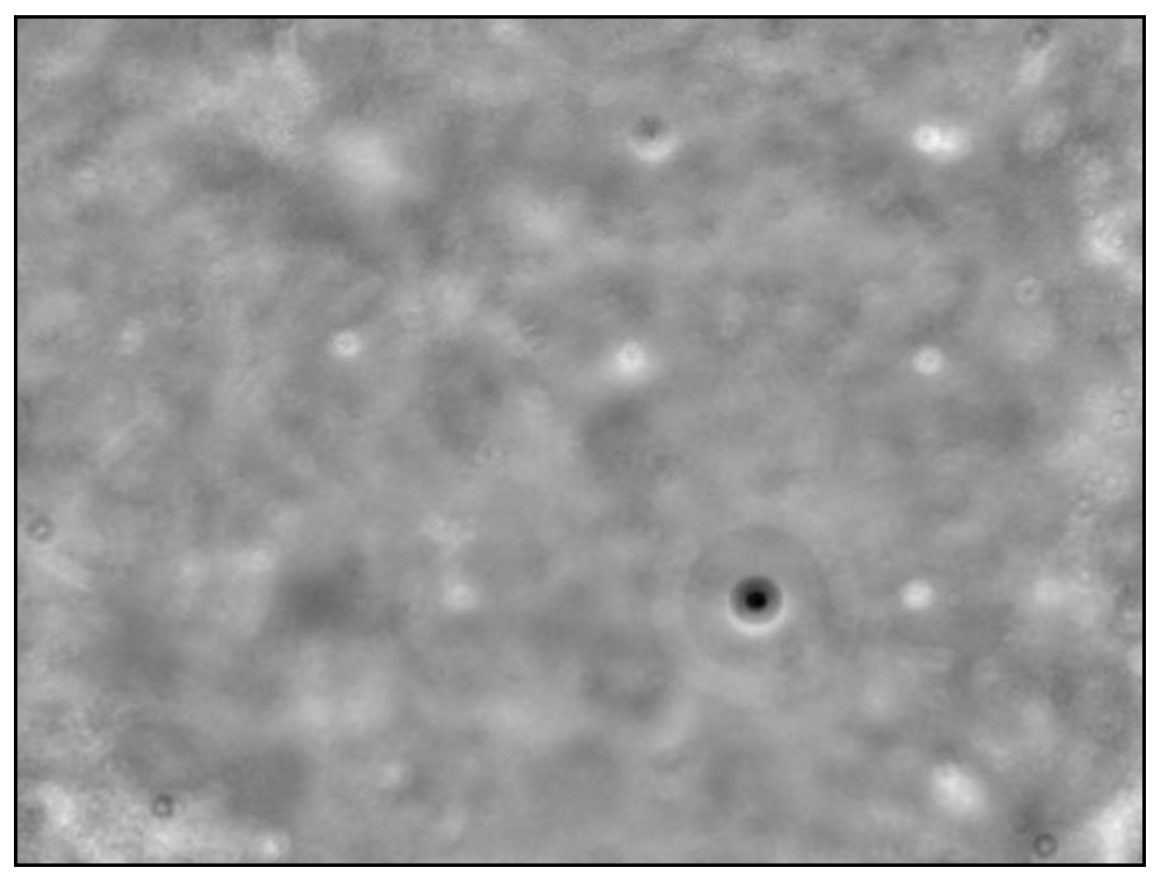

Figure 40 An example band of spatial filtered image captured by FLIR camera from the first minefield 


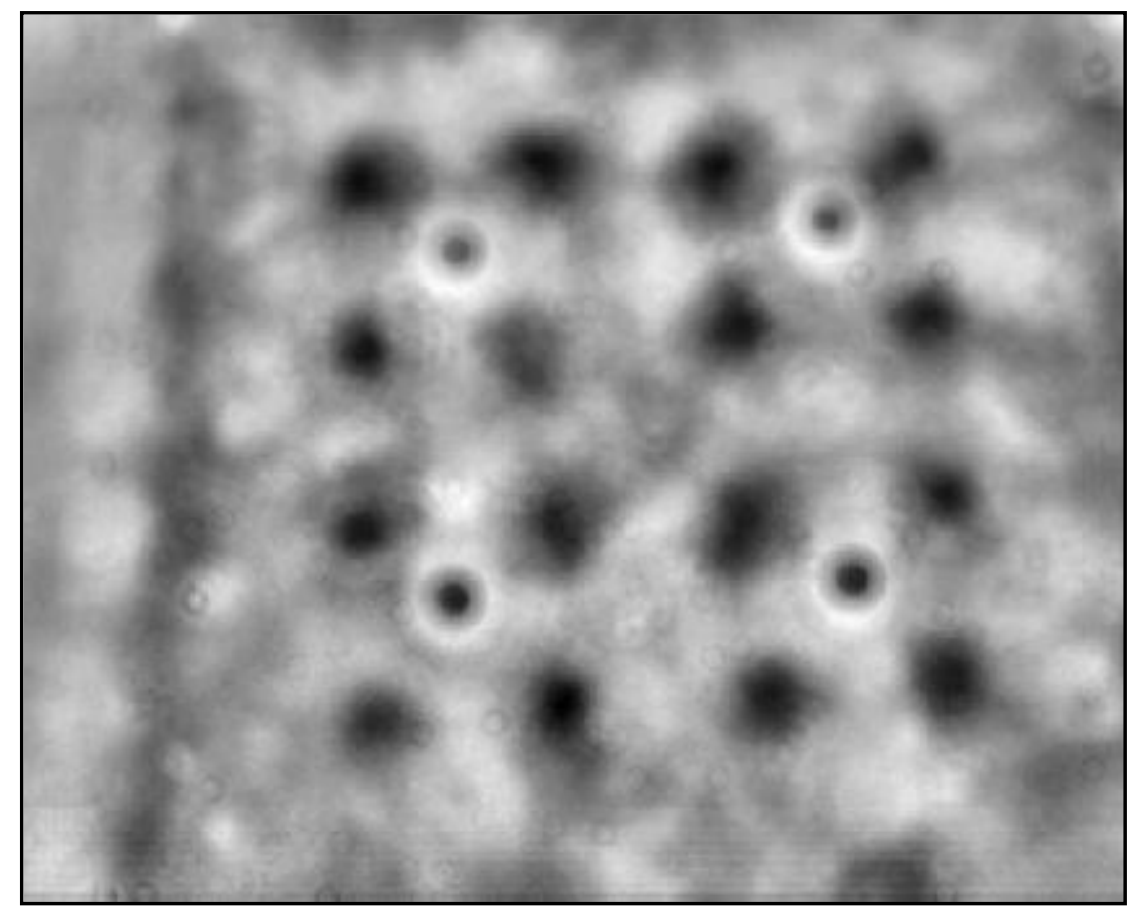

Figure 41 An example band of spatial filtered image captured by FLIR camera from the second minefield

\subsection{Classification}

The classification process is described below in four sub-steps.

\subsubsection{Preparation of the Training and the Test Sets}

First minefield consists of five classes are buried anti-tank, buried metal, buried plastic, surface plastic landmines, and soil.

Each class has different number of samples. The training set was selected randomly for each class using the ground truth. For each antipersonnel landmine PL class, 200 samples were selected, and for antitank landmine PL, 300 samples were selected. Due to abundance and variety of soil pixels, 4000 pixels were selected from the soil class. These classes, number of samples they have, and number of training samples are given in Table 6. The test set is all of the data except the training set and the gray zone pixels. 
Table 6 The class names, sample sizes, and training set sizes

\begin{tabular}{|l|l|l|}
\hline Class Name & \# of Pixels & \# of Training Pixels \\
\hline Anti-tank & 1653 & 300 \\
\hline Metal & 1350 & 200 \\
\hline Buried plastic & 732 & 200 \\
\hline Plastic & 366 & 200 \\
\hline Soil & 287975 & 4000 \\
\hline
\end{tabular}

Second minefield consists of three classes that are buried DM11, surface DM11, and soil. Training set size for each class consists of a pre-defined percentage of the class's sample size. These percentages are chosen as $1 \%$ and $3 \%$ of the sample sizes to see the effect of the training set size on classification. These classes, number of samples they have, and number of training samples are given in Table 7. Test set is all of the data except the training set and gray zone pixels.

Table 7 The class names, sample, and training set sizes for second minefield data

\begin{tabular}{|l|c|c|c|}
\hline Class Name & \# of Pixels & \#1\% of Training Pixels & \#3\% of Training Pixels \\
\hline Buried DM11 & 8014 & 81 & 243 \\
\hline Surface DM11 & 2096 & 21 & 63 \\
\hline Soil & 255964 & 2586 & 7758 \\
\hline
\end{tabular}

The training set was selected randomly from pre-defined buried and surface DM11 PLs using the second minefield's ground truth. The ground truth of the second minefield is given in Figure 42. The soil training set was selected off sites of the PLs and buffer zones. The DM11 buried PL classes training samples were taken from blue, DM11 surface PL classes red and soil class training set samples white area. These areas are shown in Figure 43. 


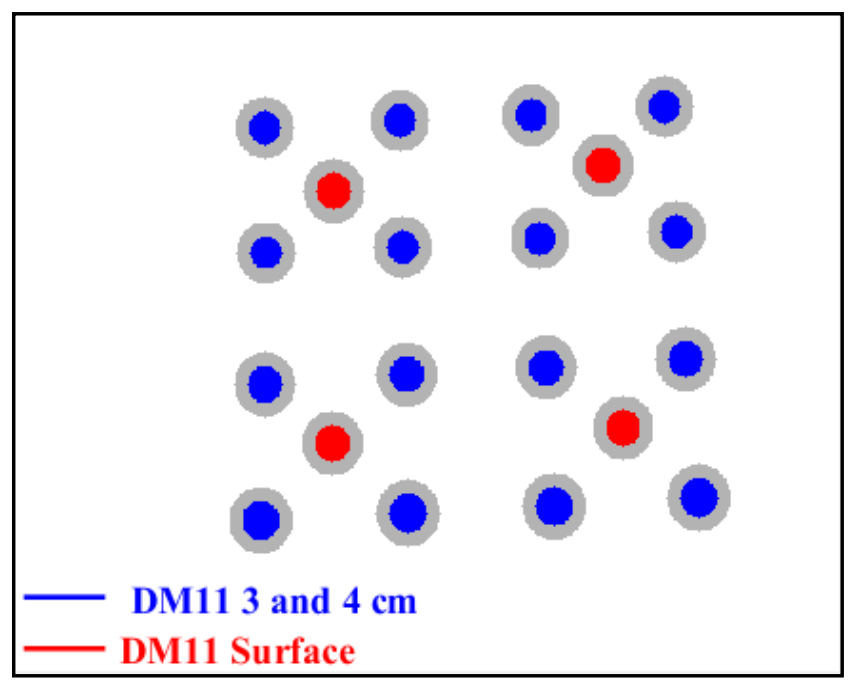

Figure 42 The second minefield's ground truth with the buffer zones

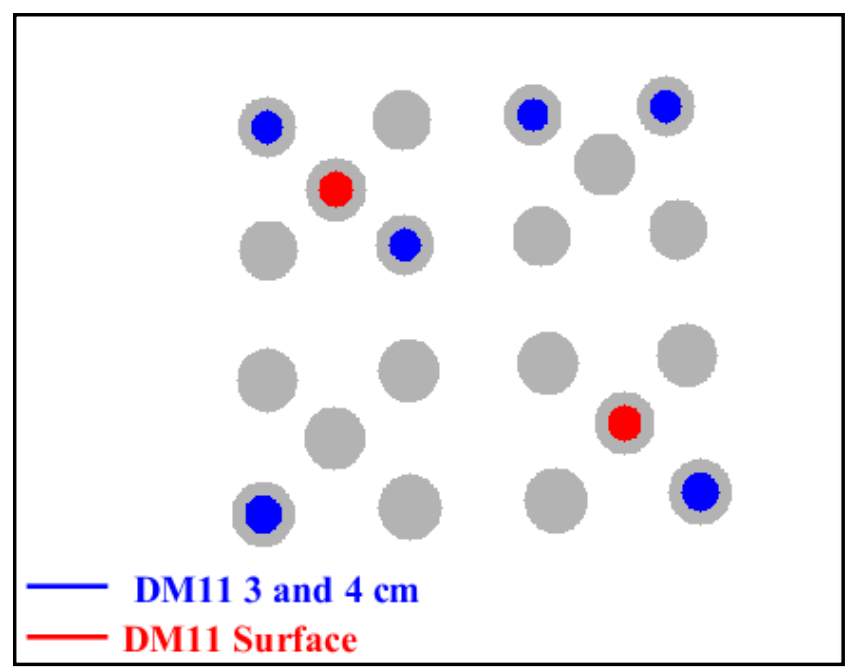

Figure 43 Training set formed version of ground truth

\subsubsection{Dimension Reduction}

To decrease the data size and processing time, KLT (also known as Principal Component Analysis, PCA) is applied and the feature size is decreased. The size of the features is determined for each classifier with the best classification performance. 


\subsubsection{Supervised Classification}

Supervised classifiers are used and compared in this study. The classifiers are listed as follows: Support Vector Machine (SVM), Mahalanobis Discriminant Analysis (MDA), and Quadratic Discriminant Analysis (QDA). K-nearest neighbor algorithm (k-NN) is used in addition to these classifiers during the last data sets classifications.

\subsubsection{Smoothing}

Post-classification smoothing is applied via a $3 \times$ 3-majority filter. When the votes for the existing label and those for another label are equal, the original label is kept. This process removes individual labels that do not correspond to a mine.

\subsubsection{Post-Processing and Detection}

Following smoothing, segments are created based on connectivity. The segments whose areas are at least twice as large as the smallest landmine, which is M14 antipersonnel PL, are accepted as detected mines in QDA, MDA and the segments whose areas are equal or larger than the smallest landmine are accepted as detected mines in SVM and kNN. 


\section{CHAPTER 7}

\section{RESULTS}

\subsection{Results for the First Data Set}

Spatially filtered images consist of thermal differences between pixels over the mine and surrounding soil pixels. Therefore, every pixel in the data reflects thermal differences rather than original thermal radiance captured by the camera. Average behavior of pixels from each class as a function of time is plotted in Figure 44.

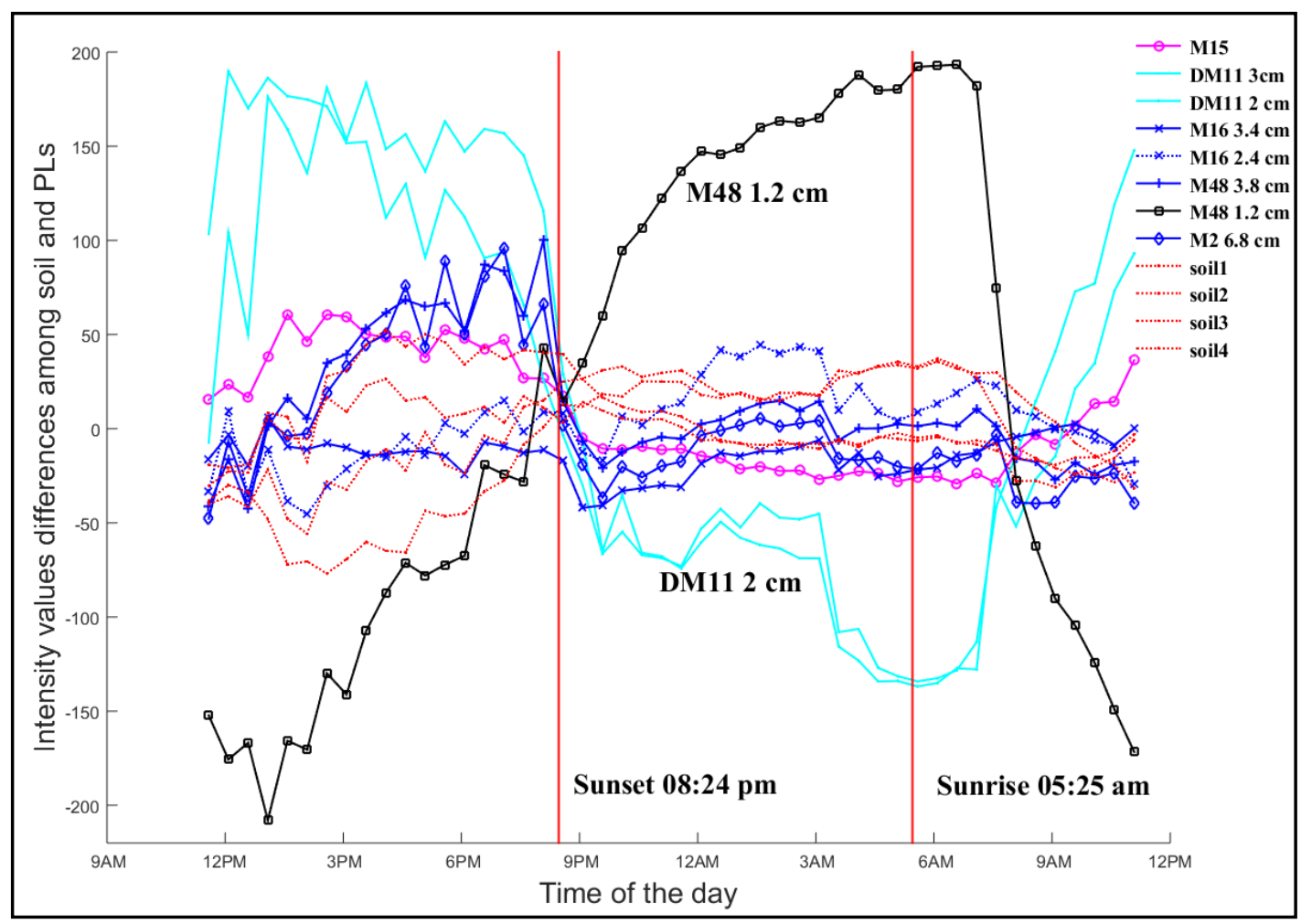

Figure 44 The average behavior of pixels from each class as a function of time 
Black line with square marker reflects the change of average thermal difference of shallowest metal mine, cyan lines without marker show buried two plastic landmines and others display anti-tank and other metal mines' thermal changes. Dashed red lines without marker reflect thermal change of four soil pixels sampled from different parts of the image. Vertical red lines specify sunset and sunrise times.

There are significant thermal differences among soil, plastic PLs, and shallowest metal PL in different hours of the day, after applying the spatial filter. The red lines (soil) go smoothly and their values are around zero. As is seen from Figure 44 especially plastic PLs can be detected using newly captured data after spatial filtering.

As a starting point, we used thermal signature of plastic PLs as a matched filter in order to find plastic PLs. Two DM11 PLs signatures were averaged and signature of DM11 PL was created. After that, this signature is convolved with spatially filtered image cube. DM11 PLs locations showed up as lighter areas in the result of this filtering. Averaged DM11 signature and the result of filtering are given in Figure 45 and Figure 46, respectively.

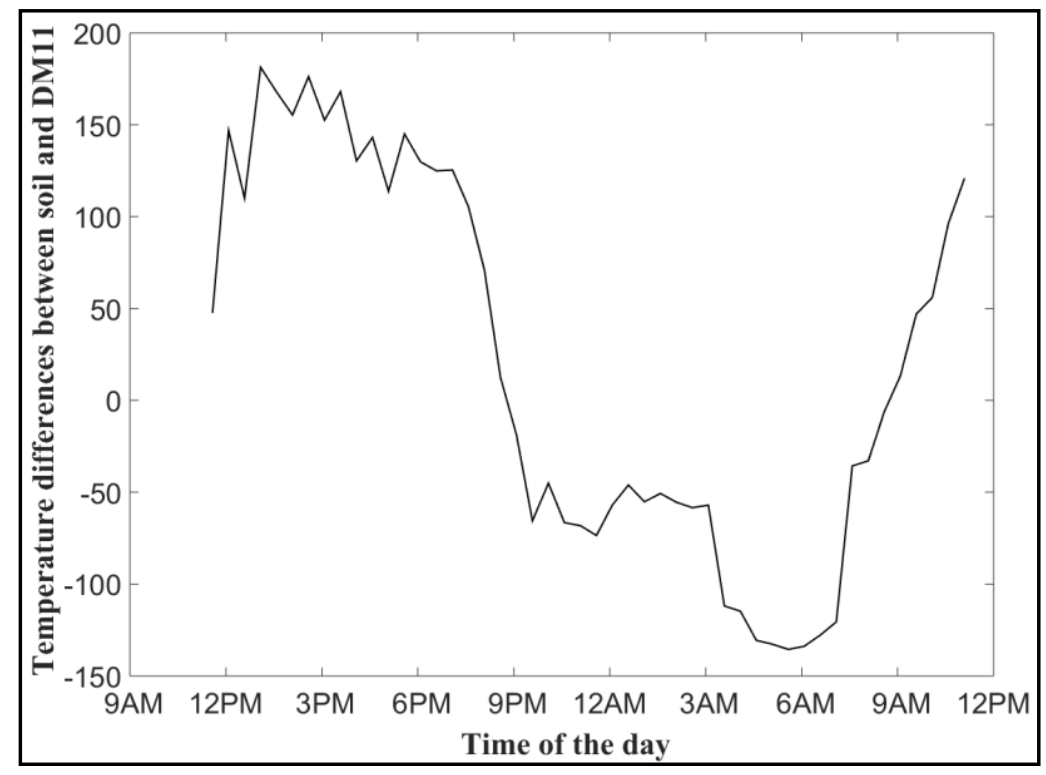

Figure 45 DM11 PL's averaged signature 


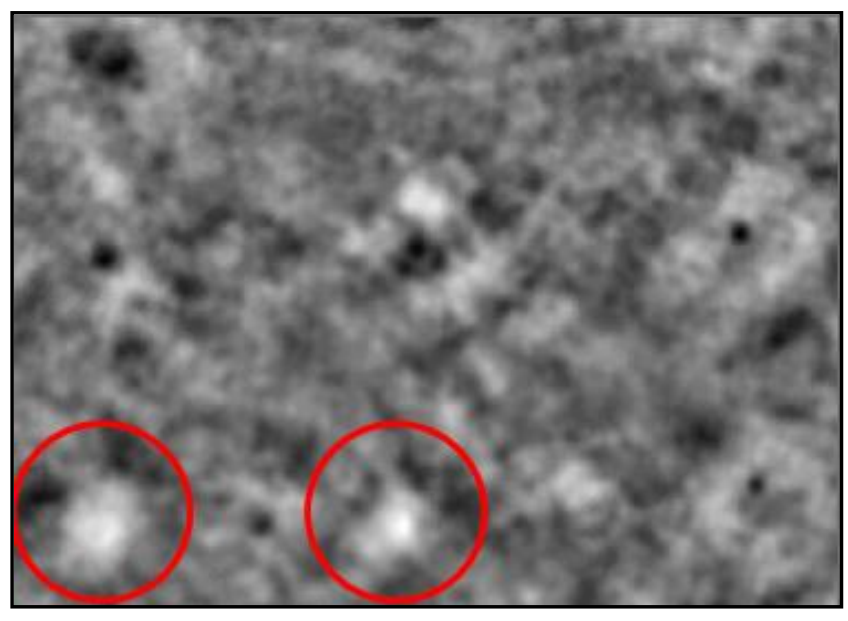

Figure 46 Result of filtering

\subsection{Second Data Sets Processing Results}

In this section, the second group of data sets, which were captured from the first minefield on 8-9 September 2015, is evaluated.

\subsubsection{Thermal Image Time Series}

We have recorded one diurnal cycle using $\mathrm{ATOM}^{\mathrm{TM}}$ and about 10 hours of data from 9 p.m. to 7 a.m. using the FLIR camera. Average behavior of pixels from each class as a function of time is plotted in Figure 47, and Figure 48. Every pixel in the data reflects thermal differences rather than original thermal radiance captured by the camera. The thermal radiance change for the same pixels in unfiltered image cube is also given in Figure 49.

Black line with square marker reflects the change of average thermal difference of shallowest metal mine, cyan lines without marker show buried two plastic landmines and others display anti-tank and other metal mines' thermal change. Dashed red lines reflect thermal change of four soil pixels sampled from different parts of the image. Vertical red lines specify sunset and sunrise times. 
Therefore, there are significant thermal differences among soil, metal mines, plastic mines, and anti-tank mine in different hours of the day, after applying the spatial filter. The red lines (soil) go smoothly and their values are around zero as expected, while metal, and anti-tank mine areas are hotter than soil pixels. On the other hand, most part of the day plastic land mines are cooler. It is observed that daytime during which solar irradiation arrives on earth is not very useful for classification but the "signatures" are quite different during nighttime. On the other hand, nighttime patterns are almost symmetrical, so recording during only first or second half of this time may be sufficient. Because of this, our recording with the FLIR camera was shorter.

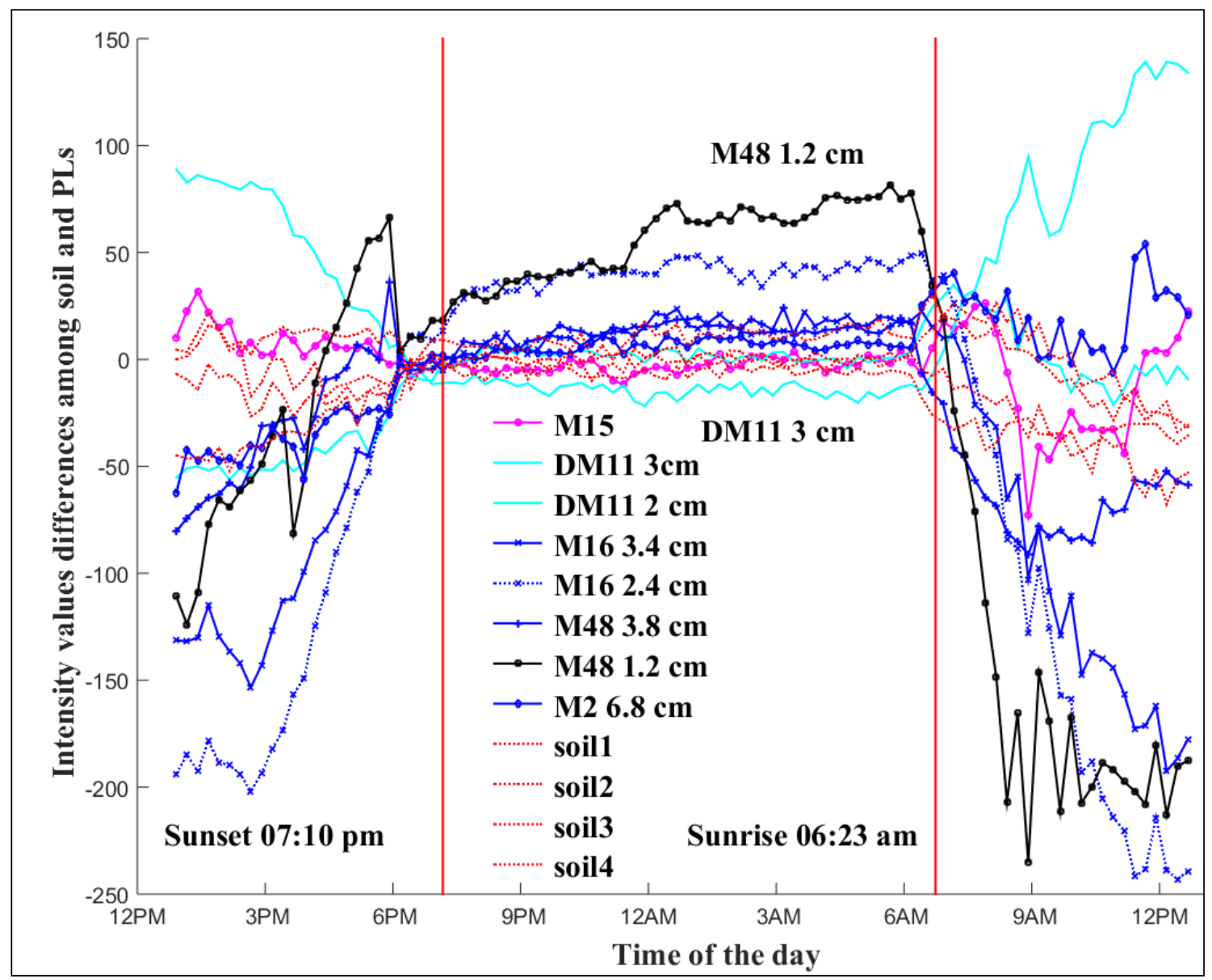

Figure 47 Diurnal thermal differences captured by $\mathrm{ATOM}^{\mathrm{TM}} 1024$ 


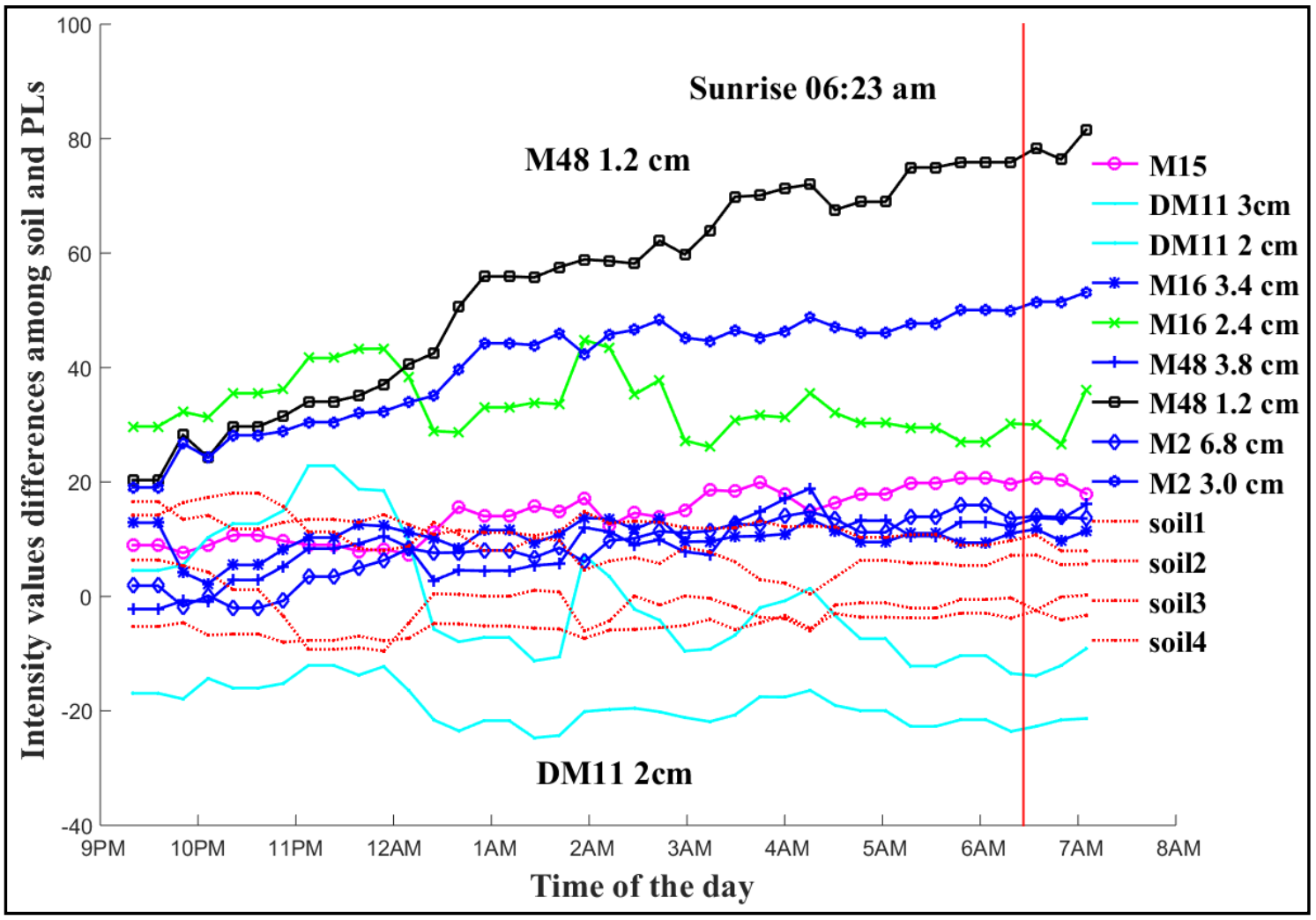

Figure 48 Intensity differences captured by FLIR T 650 SC (9:20 p.m. to 7:05 a.m.).

\subsubsection{Feature Reduction}

Data provided to classifiers are not only spatially filtered but also dimensionally reduced data. Feature sizes for each classifier are tested and selected according to the detection rate. All of the classifiers work best with feature sizes equal to or larger than 20 . For three classification methods, classification rates versus feature sizes are given in Figure 49 and Figure 50. 


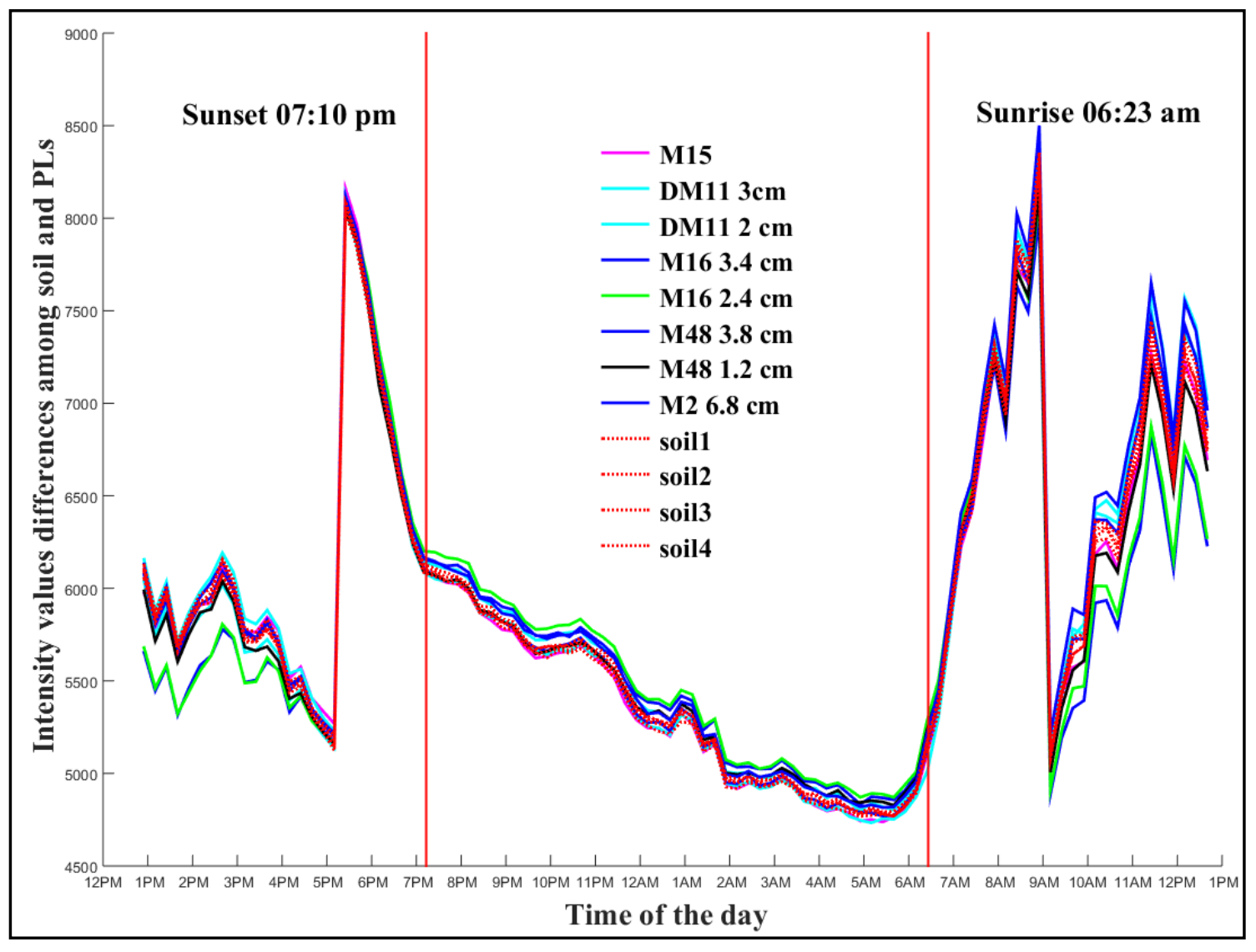

Figure 49 Diurnal original thermal radiance change captured by ATOM ${ }^{\mathrm{TM}} 1024$

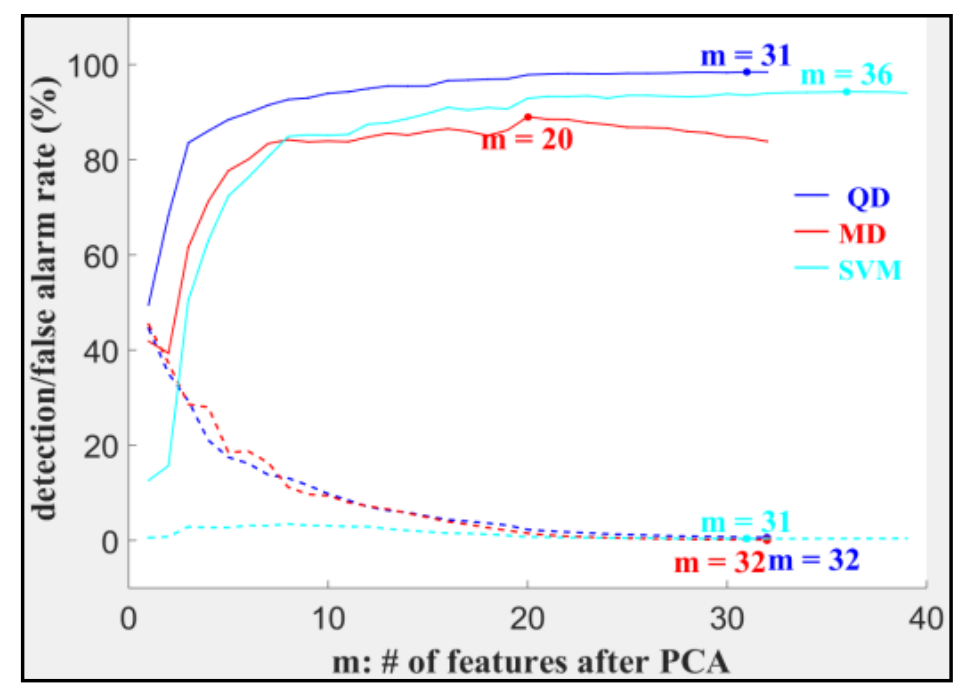

Figure 50 Detection/False Alarm Rates vs. KLT feature sizes before smoothing the classification results 


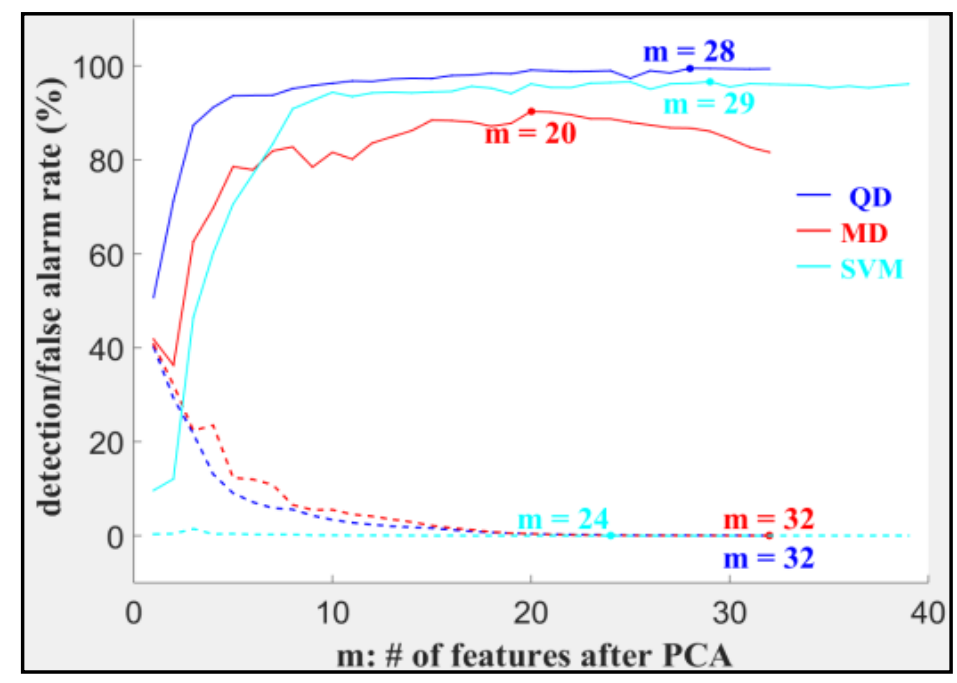

Figure 51 Detection/False Alarm Rates vs. KLT feature sizes after smoothing the classification results

\subsubsection{Classification}

The results of the three classification algorithms on the FLIR data are given in Figure 52, Figure 53 and Figure 54. After smoothing, the segments are labeled as landmine by the automated detection algorithm. The detection results are given in Figure 56, Figure 57 and Figure 58.

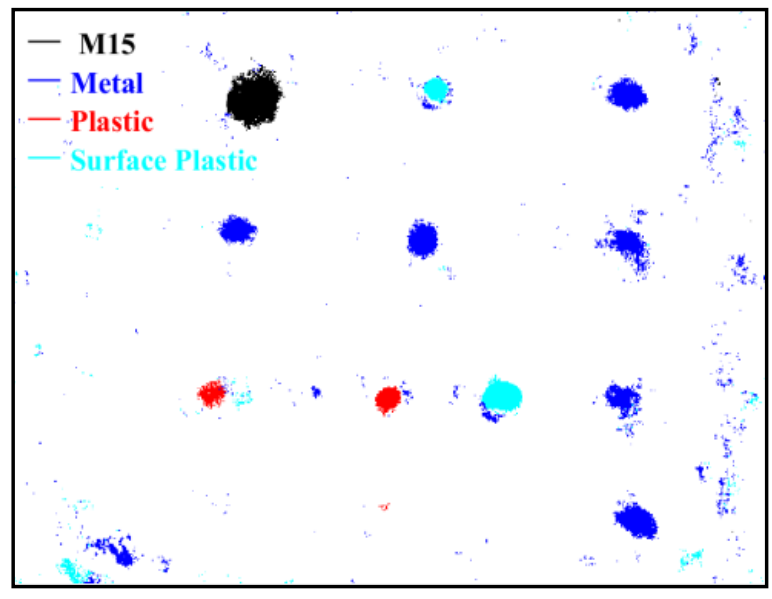

Figure 52 The MDA classification result 


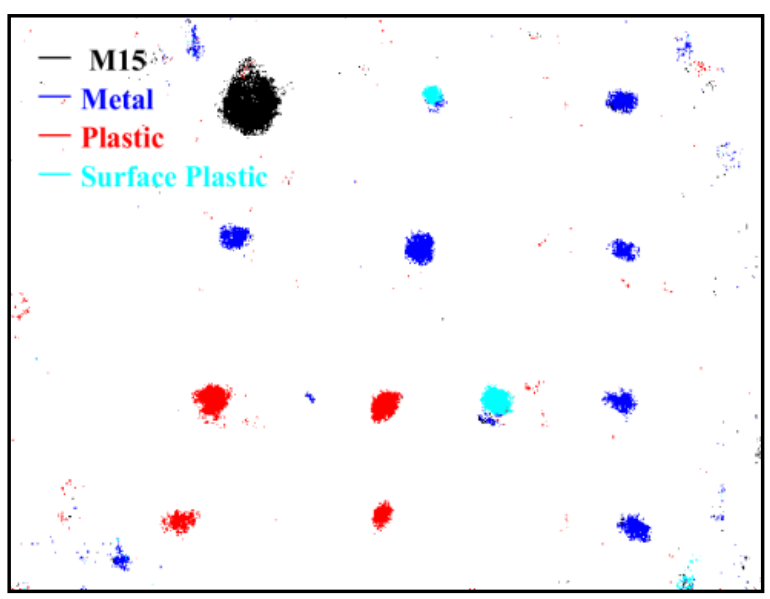

Figure 53 The SVM classification result

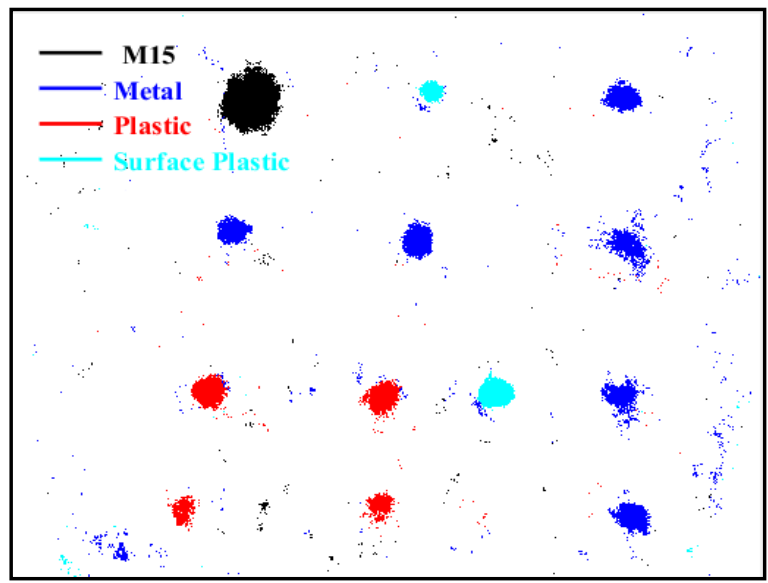

Figure 54 The QDA classification result

\subsubsection{Post Processing and Automatic Detection}

Smoothed QDA classification result is given in Figure 55 as an example. The visual improvement can easily been seen when Figure 54 and Figure 55 are compared to each other.

\subsubsection{Performance Assessment}

To evaluate the results, two main approaches are used: Pixel based evaluation and object based evaluation. Pixel based evaluation is based on correct classification of pixels with respect to the ground truth which is created by using known mine locations and radii 
given in Figure 59. To avoid transition zone contribution to the accuracy assessment, $4.5 \mathrm{~cm}$ width buffer zones are created around PLs in the ground truth, and buffer zonepixels are not taken into consideration during pixel based performance assessments.

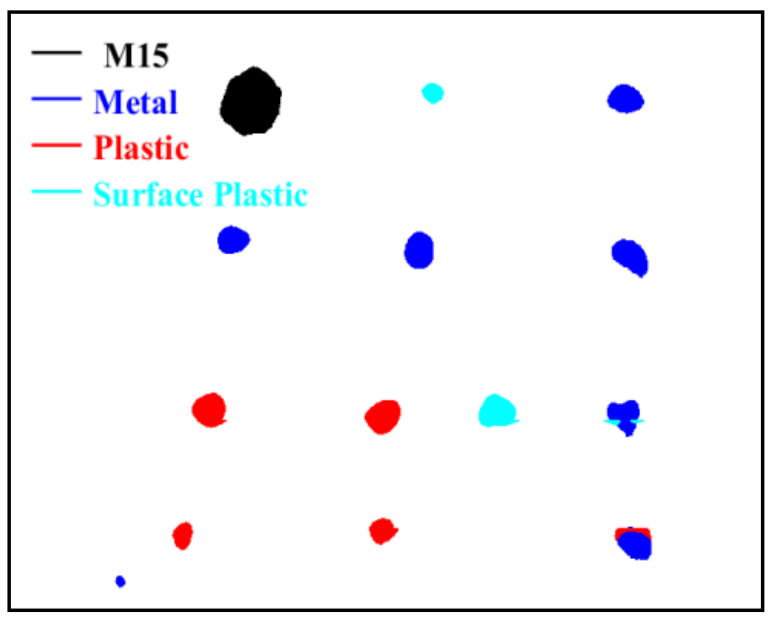

Figure 55 The smoothed QDA classification result

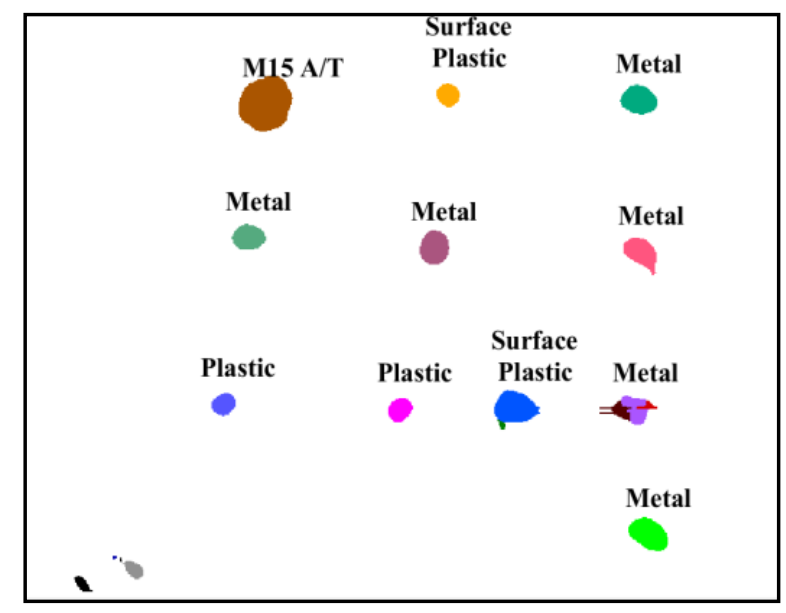

Figure 56 The MDA automatic detection result after smoothing 


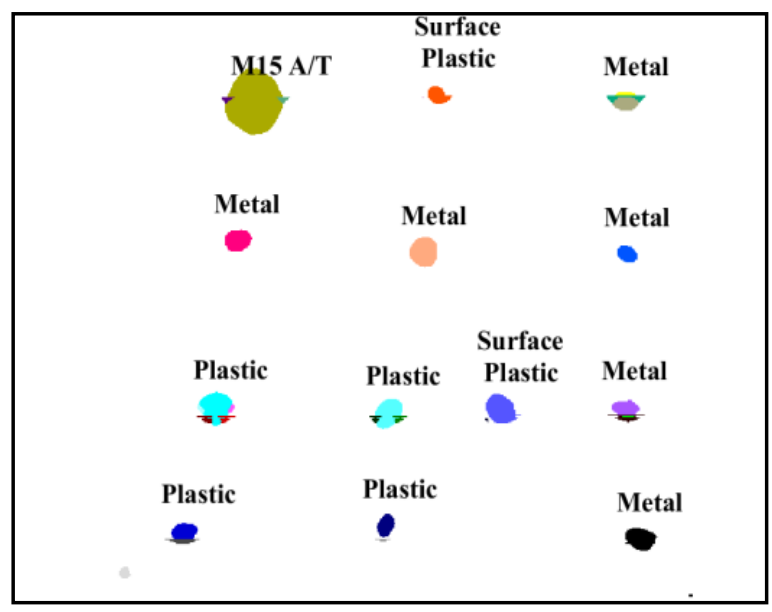

Figure 57 The SVM automatic detection result after smoothing

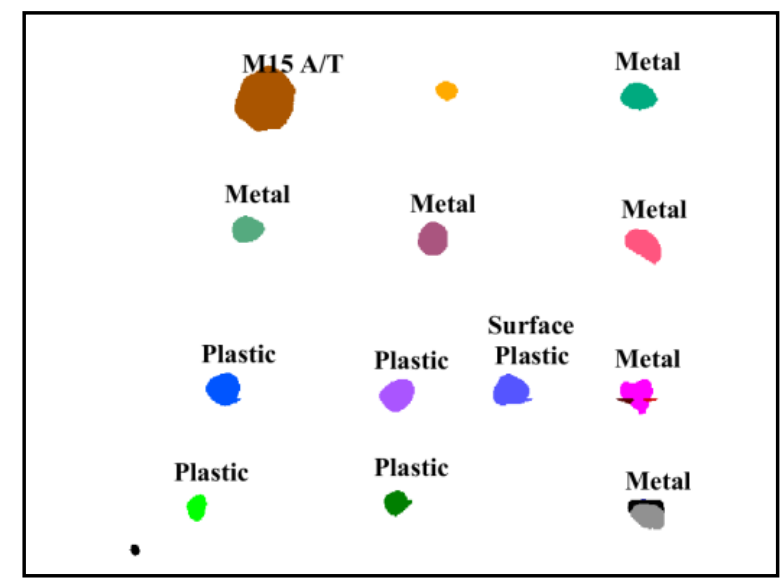

Figure 58 The QDA automatic detection result after smoothing

However, due to unbalanced sample size, correct classification rate of pixels (overall accuracy) is not a suitable metric to use. Because when whole image is classified as soil and none of the 13 mines is detected, overall accuracy rate is calculated as $99 \%$. Therefore, two metrics named detection rate and false alarm rate are utilized. Detection rate is ratio of correctly classified mine pixels to all mine pixels in the test set. This metric ignores whether soil pixels are classified correctly or not. False alarm rate is ratio of soil pixels incorrectly classified as PLs to all soil pixels in the test set. 


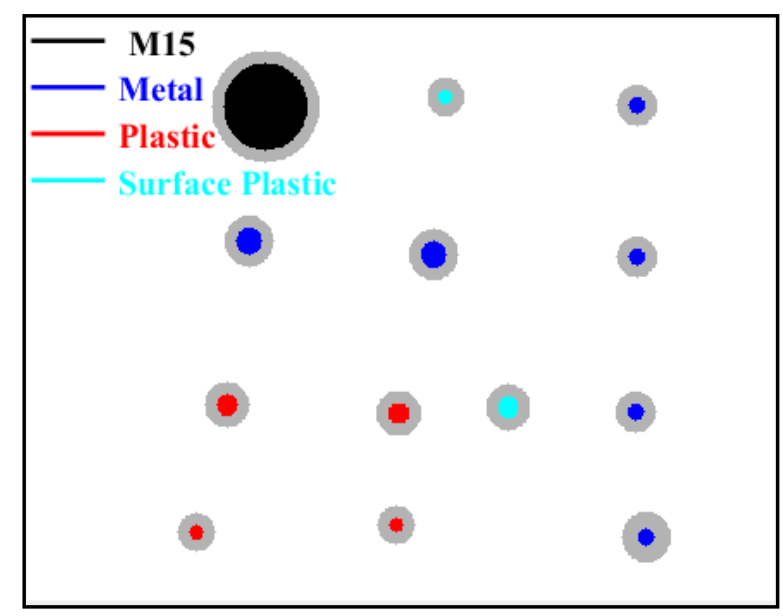

Figure 59 The ground truth with buffer zones

For the pixel-based approach, the detection rates and the false alarm rates of the classifiers are given before and after smoothing classification results given in Table 8 and Table 9. In addition, these changes are depicted in Figure 50 and Figure 51, where continuous lines represent detection rates and dashed lines represent false alarm rates as a function of the KLT feature sizes. When Figure 50 and Figure 51 are compared, it can be seen that smoothing increases the detection rates and decreases the false alarm rates.

Within all three discriminant analysis methods, QDA has the best performance with detection rate of $99 \%$ (Table 9) and false alarm rate of $0.1 \%$ (Table 9). In other words, QDA classifies the mine pixels with an accuracy of $99 \%$ and only $0.1 \%$ of the soil pixels are classified as PLs.

Table 8 Detection / False Alarm Rates of the classifiers before smoothing

\begin{tabular}{|l|c|c|}
\hline Classifier & Detection Rate (\%) & False Alarm Rate (\%) \\
\hline MDA & 89 & 1.5 \\
\hline SVM & 94 & 0.5 \\
\hline QDA & 98 & 0.7 \\
\hline
\end{tabular}


Table 9 Detection/ False Alarm Rates of the classifiers after smoothing

\begin{tabular}{|l|c|c|}
\hline Classifier & Detection Rate (\%) & False Alarm Rate $(\%)$ \\
\hline MDA & 90 & 0.4 \\
\hline SVM & 97 & 0.4 \\
\hline QDA & 99 & 0.1 \\
\hline
\end{tabular}

The other type of evaluation is based on objects detected. Detected number of mines (True Positives: TP), undetected number of mines (False Negatives: FN) and the soil detected as mine (False Positives: FP) are calculated.

Then two metrics, True Positive Rate and Precision, are calculated as follows:

True Positive Rate $=\mathrm{TP} /(\mathrm{TP}+\mathrm{FN})$

Precision $=\mathrm{TP} /(\mathrm{TP}+\mathrm{FP})$

For QDA and SVM classifications, all PLs are labeled correctly as mine and there is no FN. On the other hand for MDA classification there are two FNs (two M14 PLs), but other PLs correctly labeled as mine. TP, FP, and FN values are given in Table 10.

Table 10 True Positive, False Negative and False Positive values

\begin{tabular}{|l|c|c|c|}
\hline Classifier & TP & FN & FP \\
\hline MDA & 11 & 2 & 0 \\
\hline SVM & 13 & 0 & 0 \\
\hline QDA & 13 & 0 & 0 \\
\hline
\end{tabular}

From TP, FP and FN values, True Positive Rate (recall) and Precision are calculated and are given in Table 11. When results are analyzed, it is observed that QDA and SVM give the best results.

With these methods, all the mines are detected and no false negative exists, which is crucial in mine analysis. For pixel-based classification, QDA has the superiority over others. 
Table 11 True Positive Rate and Precision

\begin{tabular}{|l|c|c|}
\hline Classifier & True Pos. Rate (recall) (\%) & Precision (\%) \\
\hline MDA & 85 & 100 \\
\hline SVM & 100 & 100 \\
\hline QDA & 100 & 100 \\
\hline
\end{tabular}

When object based classification and pixel-based classification are taken into account together, QDA gives the best result by detecting all the PLs with the largest detecting rate and minimum false alarm rate with first minefield data and associated training sets.

\subsection{Third Data Processing Results}

Second minefield was set in order to test proposed algorithm's performance in different weather, humidity, soil, season, temperature etc. conditions with different, and decreased size of training sizes. In addition to these parameters, optimum pixelwise spatial filter diameter $R_{o}$ (outer circle diameter) and optimum sample point numbers on the inner and outer circles are investigated.

$1 \%$ and $3 \%$ of samples from classes were used as training sets respectively in order to see the performance of the proposed method with different sizes of training sets. Spatial filter's inner and outer diameters were chosen six and 50 pixels during implementations.

\subsubsection{Thermal Image Series}

We have recorded one diurnal cycle using the FLIR camera. Nighttime image cube, which was acquired between 21:00 pm to 06:45 am, was processed for detecting PLs. Average behavior of pixels from each class as a function of time is plotted in Figure 60 and Figure 61. The original thermal radiance change is given in Figure 62.

Black lines with plus markers reflect the change of average thermal difference of four surface DM11 PLs, cyan lines with circle markers show two DM11 PLs buried at four cm depth and blue lines without markers display 12 DM11 PLs' thermal change buried at three $\mathrm{cm}$ depth. Dotted red lines reflect thermal change of six soil pixels sampled from different parts of the image. Vertical red lines specify sunset and sunrise times. 
There are significant thermal differences among soil and plastic landmines, especially during the nighttime, after applying the spatial filter. In this period, DM11 PLs are cooler than soil. As we observed also in second data set, daytime during which solar irradiation arrives on earth is not very useful for classification but the "signatures" are quite different during nighttime. Thus using nighttime patterns is sufficient to detect plastic PLs as we mentioned during second data set results. In order to demonstrate this phenomenon, Thirty-nine daytime images that were shot between 12:00 pm to 03:00 pm and 08:00 am to 11:45 am were combined. The proposed algorithm was applied on daytime image cube. Comparisons of the performances were given in section 7.3.7.

\subsubsection{Feature Reduction}

Spatially filtered data provided to classifiers are dimensionally reduced. Feature sizes for each classifier are tested and selected according to the detection rate. For four classification methods, classification rates versus feature sizes are given in Figure 63 and Figure 64, where $1 \%$ of the data is used as the training set, and Figure 65 and Figure 66 , where $3 \%$ of the data is used as the training set.

\subsubsection{Classification}

The results of the four classification algorithms using the second minefield data, which was captured by the FLIR camera, are given in Figure 68, Figure 69, Figure 70, and Figure 70.

\subsubsection{Post Processing and Automatic Detection}

The segments are labeled as landmine with their class names by the automated detection algorithms after smoothing. Smoothed QDA classification result is given in Figure 71 and detection results are given in Figure 72, Figure 73, Figure 74 and Figure 75. 


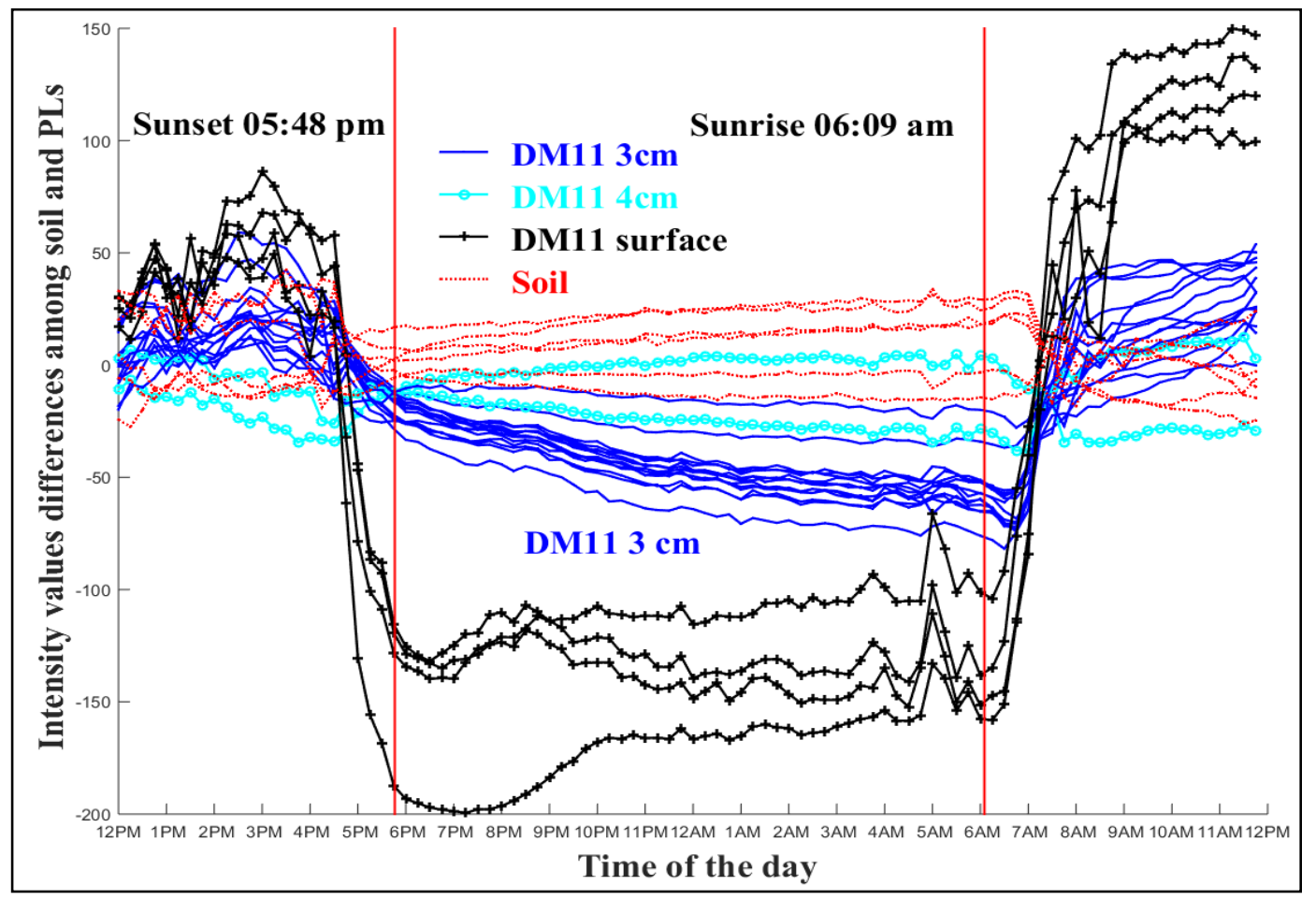

Figure 60 Diurnal thermal differences captured by FLIR

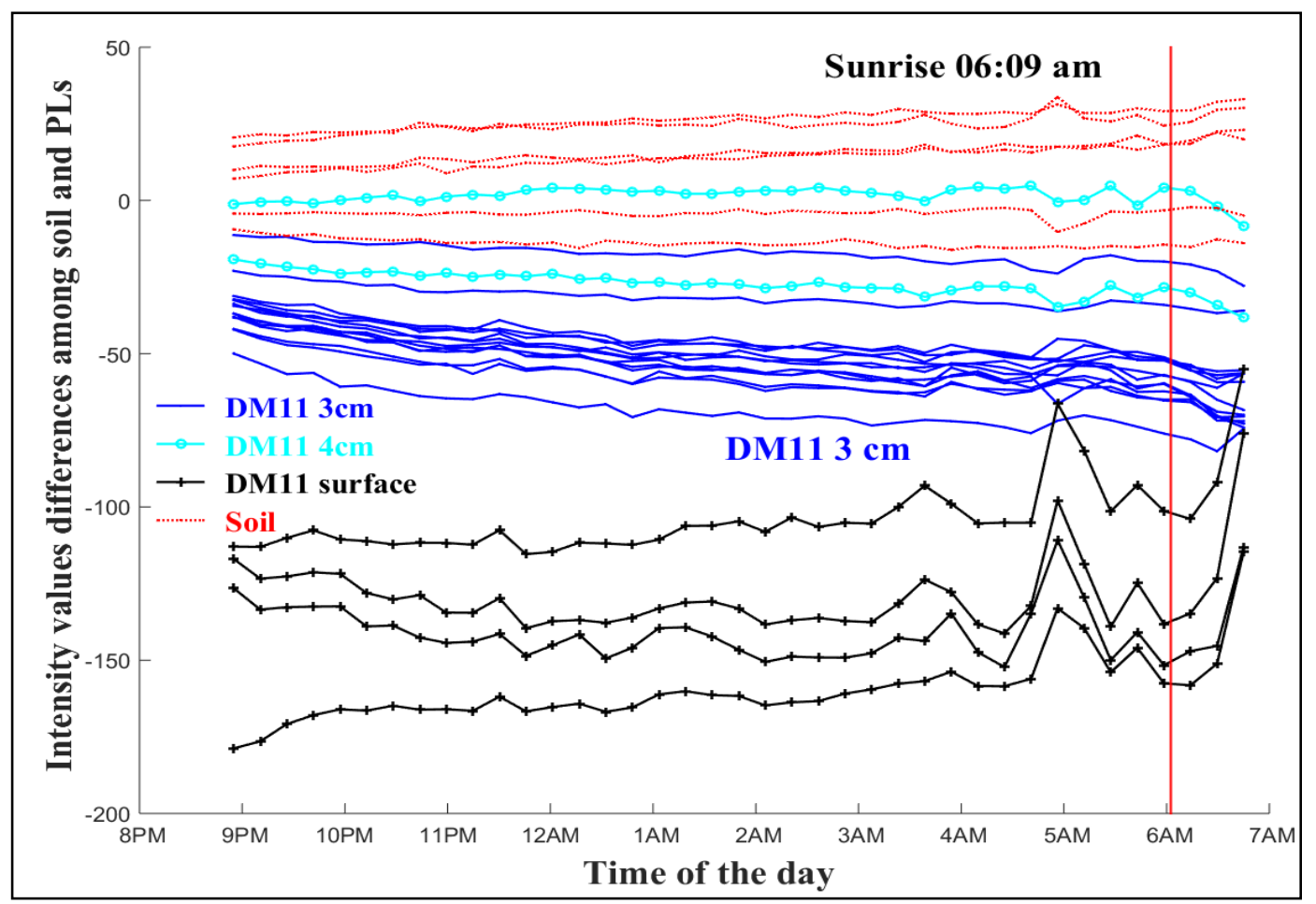

Figure 61 Intensities differences captured by FLIR (9:00 p.m. to 06:45 a.m.). 


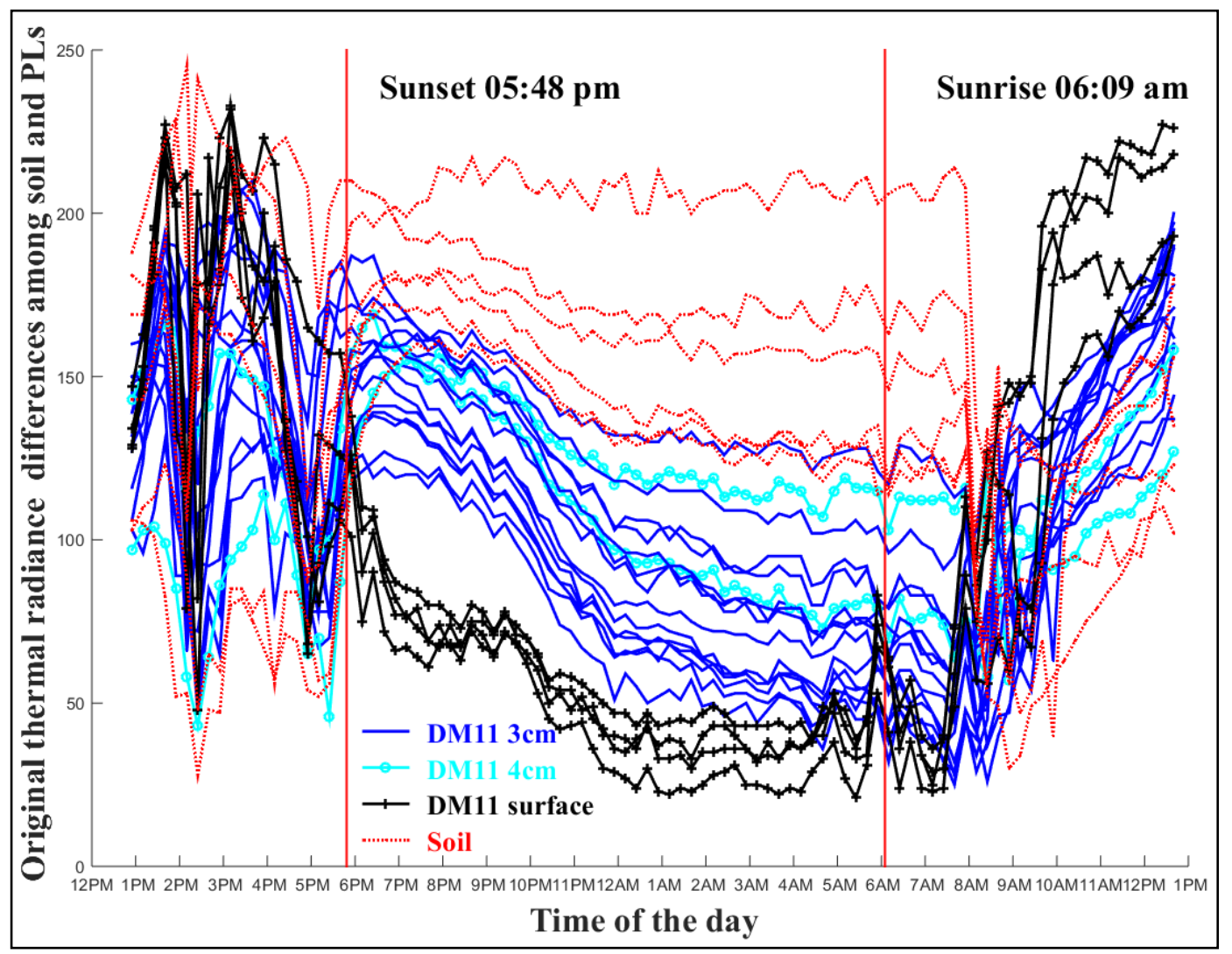

Figure 62 Original thermal radiance change for the $3^{\text {th }}$ data set

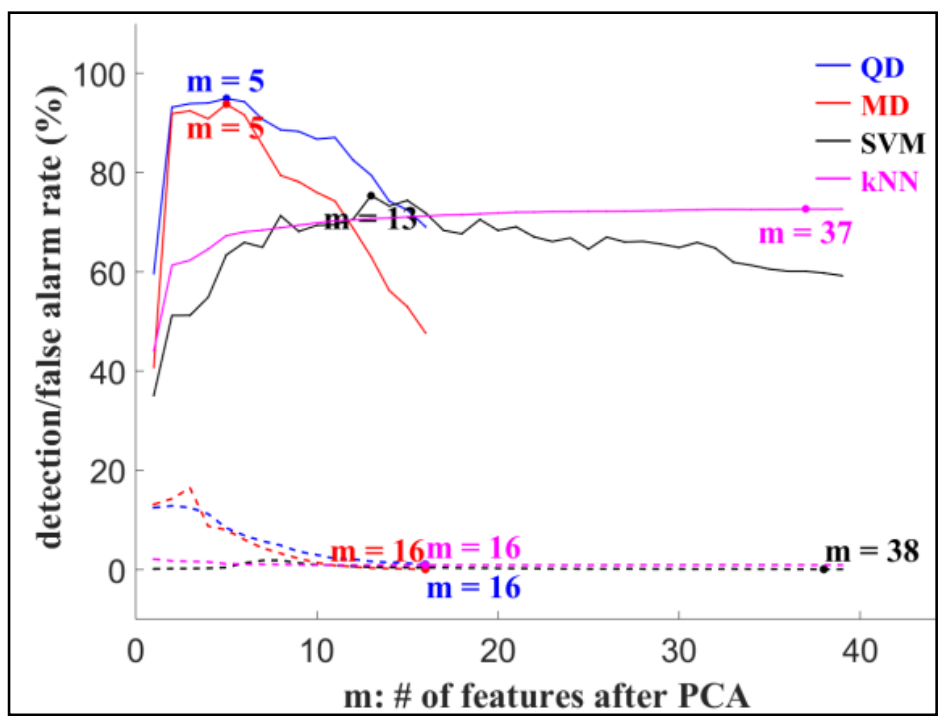

Figure 63 Detection/False Alarm Rates vs. KLT feature sizes before smoothing the classification results with $1 \%$ training set 


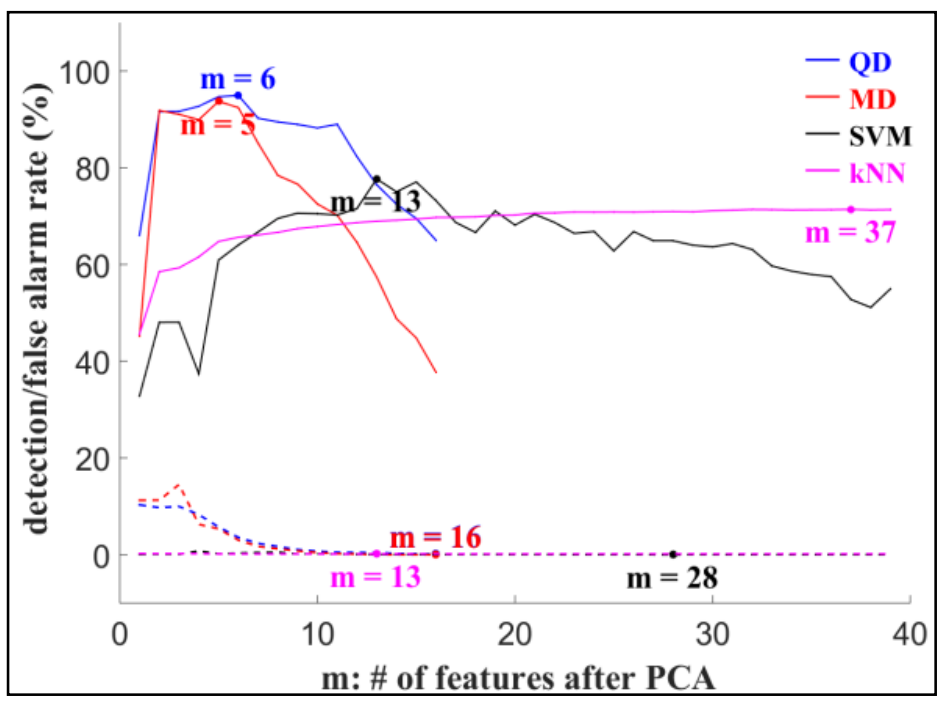

Figure 64 Detection/False Alarm Rates vs. KLT feature sizes after smoothing the classification results with $1 \%$ training set

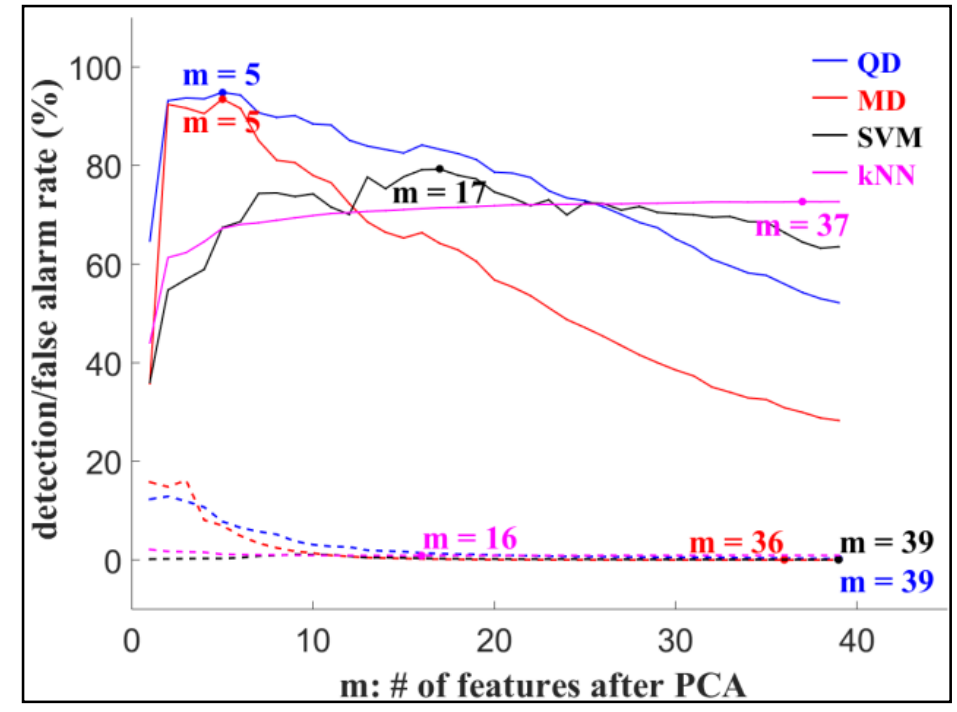

Figure 65 Detection/False Alarm Rates vs. KLT feature sizes before smoothing the classification results with $3 \%$ training set 


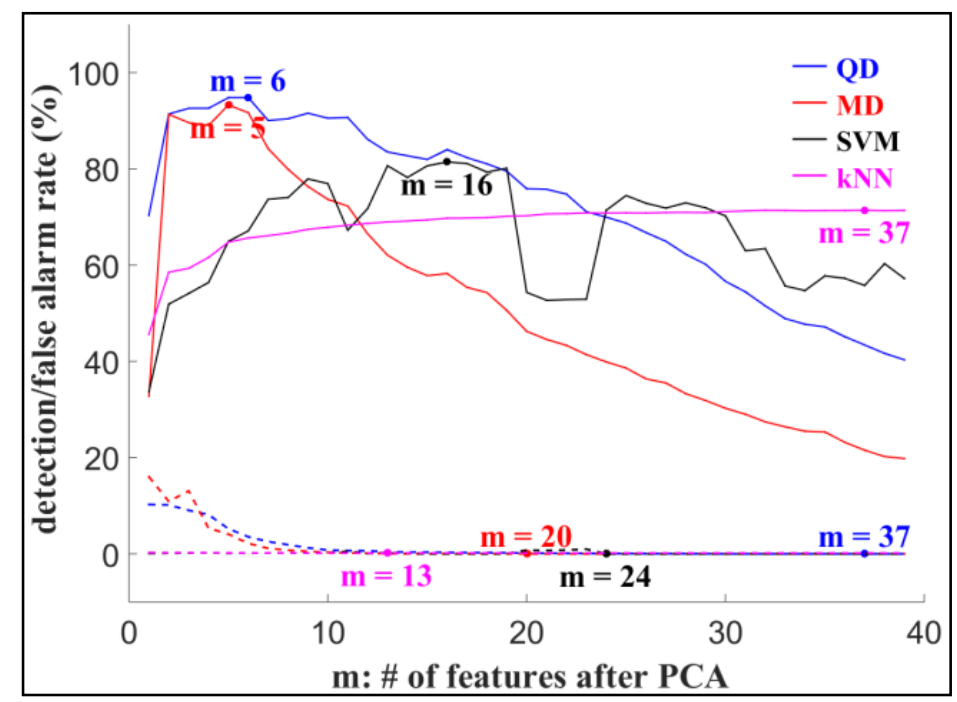

Figure 66 Detection/False Alarm Rates vs. KLT feature sizes after smoothing the classification results with $3 \%$ training set

\subsubsection{Performance Assessment}

Pixel-based evaluation and object-based evaluation were performed in order to evaluate the results for the first landmine data set. During the performance assessment of the second landmine, $3 \%$ of the samples from classes were used as the training set.

To avoid transition zone contribution to the accuracy assessment in which deciding for the ground truth is subjective, $4.5 \mathrm{~cm}$ width buffer zones are created around PLs in the ground truth, and buffer zone-pixels are not taken into consideration during pixel based performance assessments as performed earlier. 


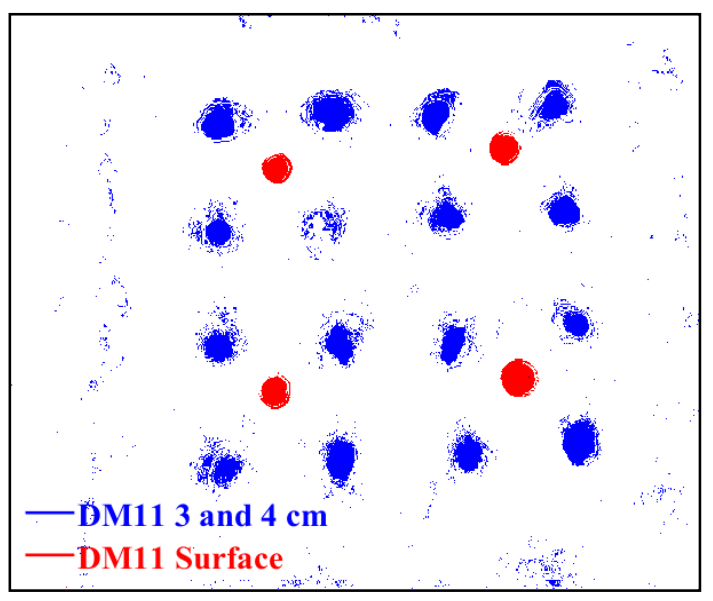

Figure 67 The k-NN classification result before smoothing

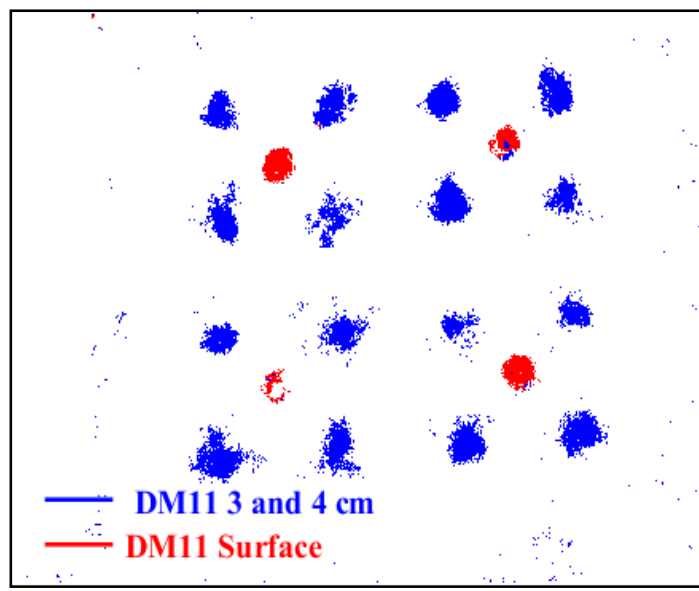

Figure 68 The SVM classification result before smoothing

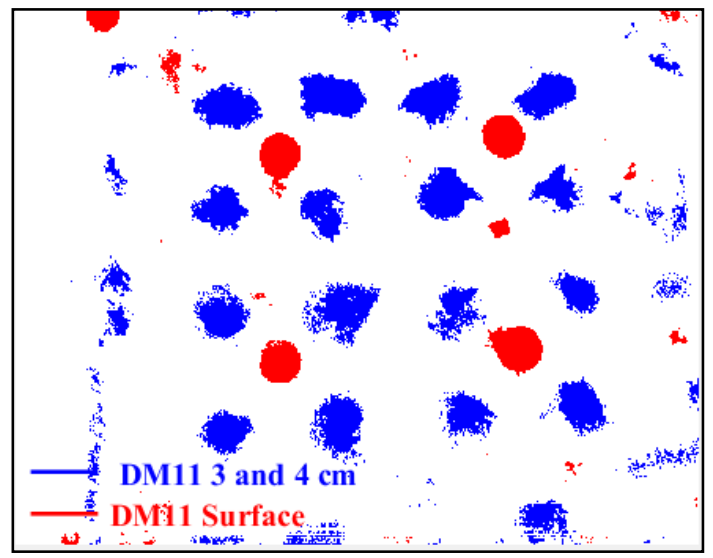

Figure 69 The MDA classification result 


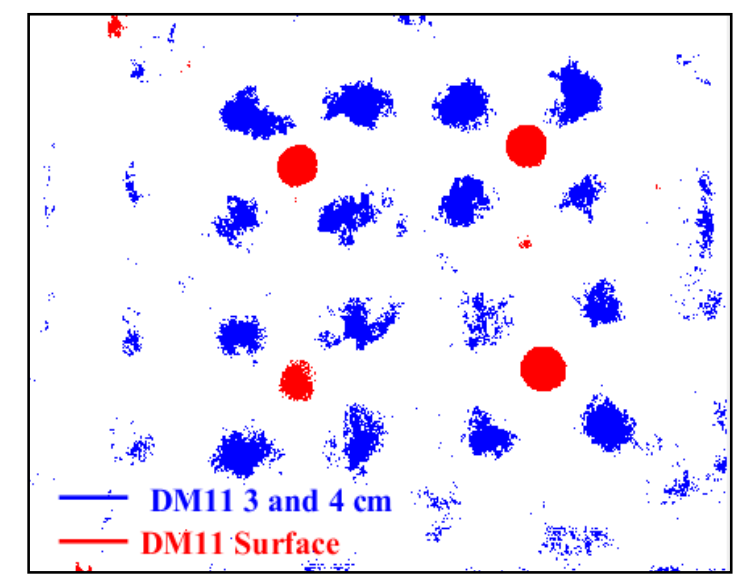

Figure 70 The QDA classification result before smoothing

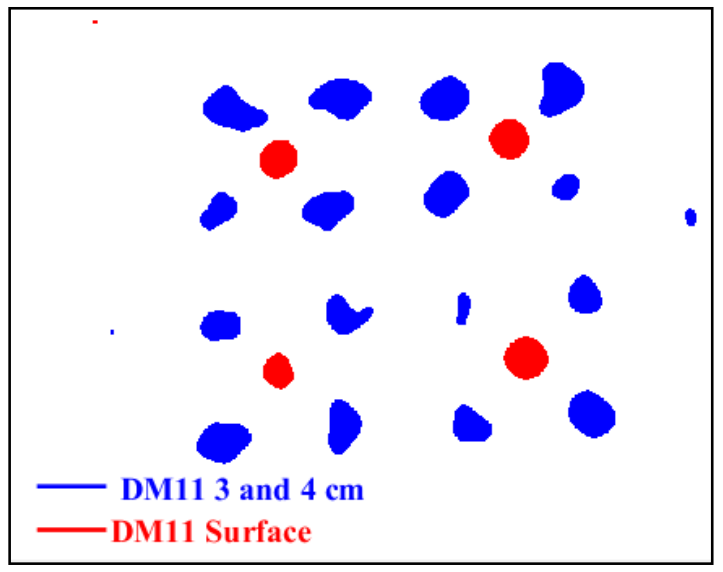

Figure 71 The smoothed QDA classification result

Smoothing the classification results before segmentation, and enlarging training set do not promote the detection rates for QDA and MDA. However, SVM's detection rates get better. Smoothing the classification result before segmentation does not contribute positive effect on k-NN detection result; however, enlarging training set increases the detection results of k-NN. On the other hand, false alarm rates decrease with enlarging training set size for all algorithms. Change of the detection and false alarm rates for two different training set sizes are given in Table 12 and Table 13, respectively. Change of the detection and false alarm rates for two different training set sizes after smoothing are given in Table 14. 
Even though larger detection rates can be obtained with QDA and MDA, false alarm rates are also larger than SVM's and k-NN's false alarm rates at the same feature sizes that are five and six. Therefore, SVM and k-NN false alarm rate levels are more stable and near zero for all feature sizes. When false alarm rates and detection rates are considered together, optimum feature size is within the range of 7 to 11 for MDA and QDA. The feature size that gives the maximum detection rate can be accepted as the optimum feature size for SVM and k-NN because of stable and low false alarm rates of SVM and k-NN. Optimum feature sizes are given with detection rates and false alarm rates in Table 15.

Table 12 Detection and false alarm rates chance with smoothing in $1 \%$ training set

\begin{tabular}{|l|c|c|c|c|}
\hline Classifier & $\begin{array}{c}\text { Max. detection } \\
\text { rate }(\%), \\
\text { before } \\
\text { smoothing }\end{array}$ & $\begin{array}{c}\text { Max. detection } \\
\text { rate }(\%), \\
\text { after } \\
\text { smoothing }\end{array}$ & $\begin{array}{c}\text { False alarm rate } \\
(\%), \\
\text { before } \\
\text { smoothing }\end{array}$ & $\begin{array}{c}\text { False alarm rate } \\
(\%), \\
\text { after smoothing }\end{array}$ \\
\hline k-NN & 62 & 57 & 1.2 & 0.1 \\
\hline SVM & 75 & 78 & 0.5 & 0 \\
\hline MDA & 94 & 94 & 8 & 5 \\
\hline QDA & 95 & 95 & 8 & 4 \\
\hline
\end{tabular}

Table 13 Detection and false alarm rates chance with smoothing in $3 \%$ training set

\begin{tabular}{|l|c|c|c|c|}
\hline Classifier & $\begin{array}{c}\text { Max. detection } \\
\text { rate }(\%), \\
\text { before } \\
\text { smoothing }\end{array}$ & $\begin{array}{c}\text { Max. detection } \\
\text { rate }(\%), \\
\text { after } \\
\text { smoothing }\end{array}$ & $\begin{array}{c}\text { False alarm rate } \\
(\%), \\
\text { before } \\
\text { smoothing }\end{array}$ & $\begin{array}{c}\text { False alarm rate } \\
(\%), \\
\text { after smoothing }\end{array}$ \\
\hline k-NN & 73 & 71 & 0.9 & 0.1 \\
\hline SVM & 79 & 81 & 0.3 & 0 \\
\hline MDA & 93 & 93 & 7 & 4 \\
\hline QDA & 95 & 95 & 8 & 3 \\
\hline
\end{tabular}


Table 14 Detection/false alarm rates change after smoothing wrt to training sizes

\begin{tabular}{|l|c|c|c|c|}
\hline Classifier & $\begin{array}{c}\text { Max. detection } \\
\text { rate }(\%), \\
1 \% \text { training set }\end{array}$ & $\begin{array}{c}\text { Max. detection } \\
\text { rate }(\%),\end{array}$ & $\begin{array}{c}\text { False alarm rate } \\
(\%), \\
1 \% \text { training set }\end{array}$ & $\begin{array}{c}\text { False alarm rate } \\
(\%), \\
\text { traing set }\end{array}$ \\
\hline k-NN & 57 & 71 & 0.1 & 0.1 \\
\hline SVM & 79 & 81 & 0 & 0 \\
\hline MDA & 94 & 93 & 5 & 4 \\
\hline QDA & 95 & 95 & 4 & 3 \\
\hline
\end{tabular}

Table 15 Optimum feature sizes

\begin{tabular}{|l|c|c|c|}
\hline Classifier & $\begin{array}{c}\text { Optimum } \\
\text { feature size }\end{array}$ & $\begin{array}{c}\text { Detection rate (\%) } \\
\text { 3\% training set }\end{array}$ & $\begin{array}{c}\text { False alarm rate (\%), } \\
\text { 3\% training set }\end{array}$ \\
\hline k-NN & 37 & 71 & 0.1 \\
\hline SVM & 16 & 81 & 0 \\
\hline MDA & 7 & 84 & 1.2 \\
\hline QDA & 11 & 90 & 0.7 \\
\hline
\end{tabular}

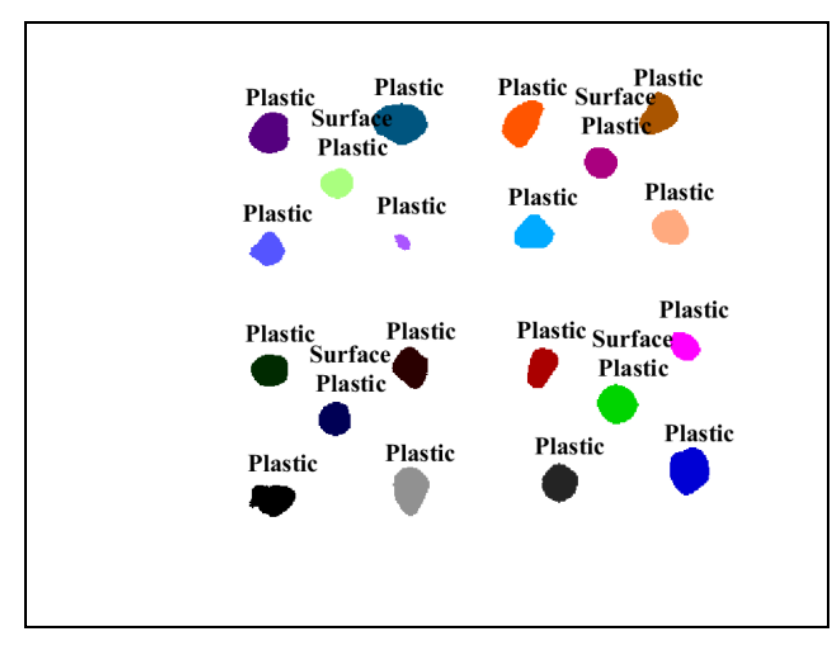

Figure 72 The k-NN automatic detection result 


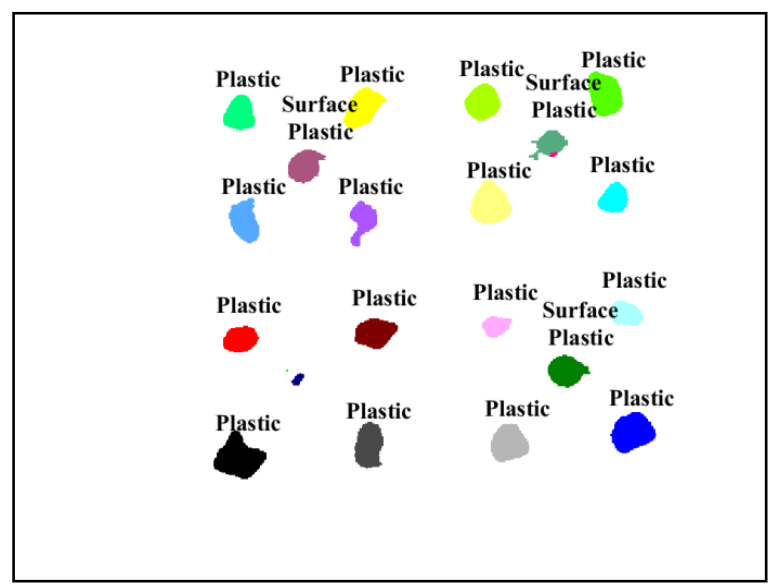

Figure 73 The SVM automatic detection result

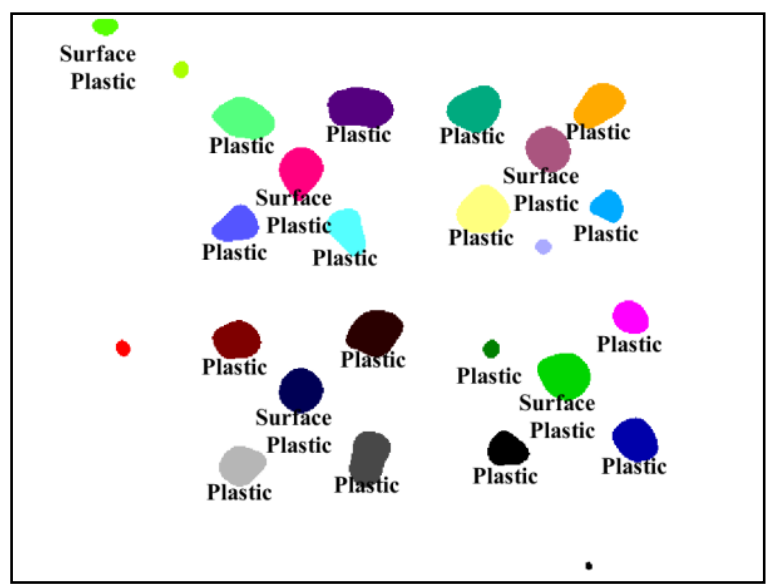

Figure 74 MDA automatic detection result

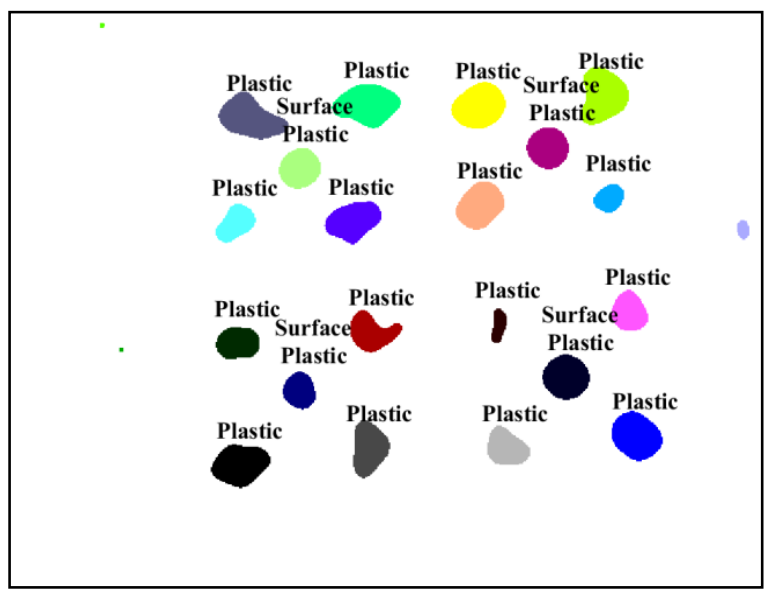

Figure 75 The QDA automatic detection result. 
Table 16 True Positive, False Negative and False Positive values.

\begin{tabular}{|l|c|c|c|}
\hline Classifier & \multicolumn{1}{|l|}{ TP } & FN & FP \\
\hline k-NN & 20 & 0 & 0 \\
\hline SVM & 19 & 1 & 0 \\
\hline MDA & 20 & 0 & 1 \\
\hline QDA & 20 & 0 & 0 \\
\hline
\end{tabular}

From TP, FP and FN values, True Positive Rate (recall) and Precision are calculated and are given in Table 17. When results are analyzed, it is observed that QDA and k-NN (k $=1$ ) give the best results.

When object-based classification and pixel-based classification are taken into account together, QDA gives the best result by detecting all the PLs with the largest detecting rate. SVM worked with minimum false alarm rates. In addition, SVM missed a PL and it is critical for mine cleaning on the spot. The k-NN algorithm gives the best results when the $\mathrm{k}$ parameter is selected as one (Figure 76).

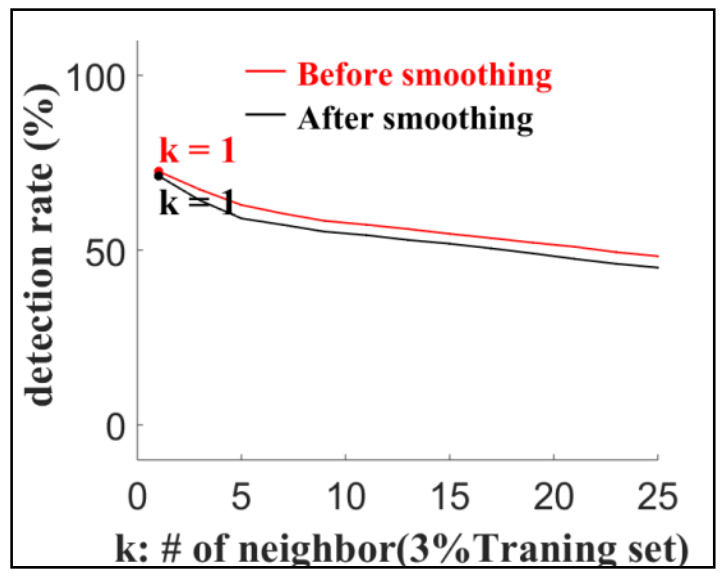

Figure 76 Detection rates \& k

Table 17 True Positive Rate and Precision

\begin{tabular}{|l|c|c|}
\hline Classifier & True Pos. Rate (recall) $(\%)$ & Precision $(\%)$ \\
\hline k-NN & 100 & 100 \\
\hline SVM & 95 & 100 \\
\hline MDA & 100 & 95 \\
\hline QDA & 100 & 100 \\
\hline
\end{tabular}




\subsubsection{Optimization of Spatial Filter}

Different $\boldsymbol{R}_{\boldsymbol{o}}$ sizes and sample point numbers influence on the QDA classification results were experienced on second minefield data with $3 \%$ training set in order to get an idea of the optimum spatial filter size.

Firstly, sample-point number is fixed to 48 and $\boldsymbol{R}_{\boldsymbol{o}}$ size is changed to calculate the optimum $\boldsymbol{R}_{\boldsymbol{o}}$ size. These different outer radii were $15(5.1 \mathrm{~cm}), 30(10.2 \mathrm{~cm}), 50(17 \mathrm{~cm})$ and $70(23.8 \mathrm{~cm})$ pixels. The classification detection rates and false alarm rates before and after smoothing are given in Table 18 and Table 19, respectively, with respect to spatial filter outer radius sizes.

These results' plots can be seen Figure 77 and Figure 78 as well. The algorithm gives the best result with 50 pixel-size spatial filter that corresponds to two times DM11 diameter $(8.1 \mathrm{~cm}, 24$ pixels). This size of the filter leads to maximum detection rates and minimum false alarm rates compared to other solutions. In addition, the positive effect of smoothing on decreasing false alarm rates can be seen clearly from Table 18, Table 19 and Figure 77, Figure 78 as well.

Table 18 Rate results with various $R_{o}$ before smoothing classification results

\begin{tabular}{|l|c|c|c|}
\hline Classifier & $\begin{array}{c}R_{o} \\
\text { (pixels) }\end{array}$ & $\begin{array}{c}\text { Max. detection rate (\%), } \\
3 \% \text { training set }\end{array}$ & $\begin{array}{c}\text { False alarm rates (\%), } \\
\text { at max. detection rate points }\end{array}$ \\
\hline \multirow{4}{*}{ QDA } & 15 & 79 & 21 \\
\cline { 2 - 4 } & 30 & 91.5 & 10.4 \\
\cline { 2 - 4 } & $\mathbf{5 0}$ & $\mathbf{9 5}$ & $\mathbf{7 . 8}$ \\
\cline { 2 - 4 } & 70 & 94 & 5.4 \\
\hline
\end{tabular}

Table 19 Rate results with various $R_{o}$ after smoothing classification results

\begin{tabular}{|l|c|c|c|}
\hline Classifier & $\begin{array}{c}R_{o} \\
\text { (pixels) }\end{array}$ & $\begin{array}{c}\text { Max. detection rate (\%), } \\
\text { 3\% training set }\end{array}$ & $\begin{array}{c}\text { False alarm rates (\%), } \\
\text { at max. detection rate points }\end{array}$ \\
\hline \multirow{4}{*}{ QDA } & 15 & 83 & 15 \\
\cline { 2 - 4 } & 30 & 92 & 5 \\
\cline { 2 - 4 } & $\mathbf{5 0}$ & $\mathbf{9 5}$ & $\mathbf{3 . 5}$ \\
\cline { 2 - 4 } & 70 & 94 & 5.4 \\
\hline
\end{tabular}




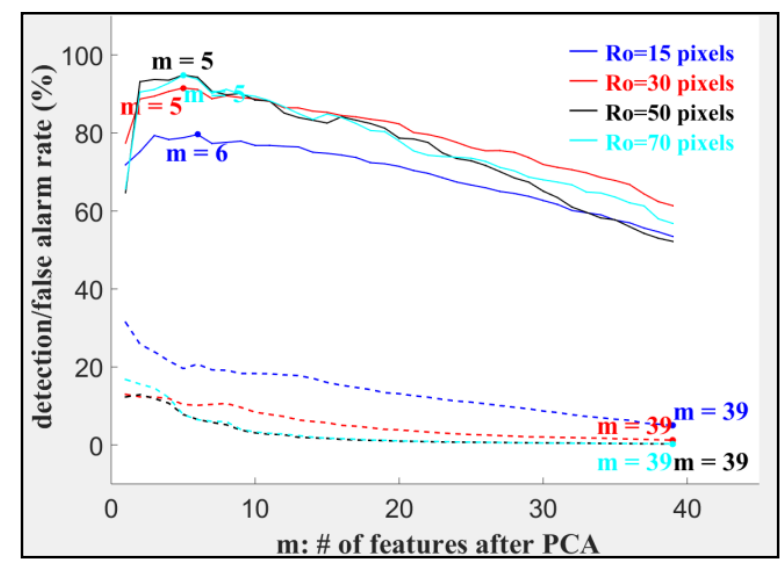

Figure 77 Detection/False Alarm Rates vs. KLT feature sizes before smoothing the classification results with $3 \%$ training set

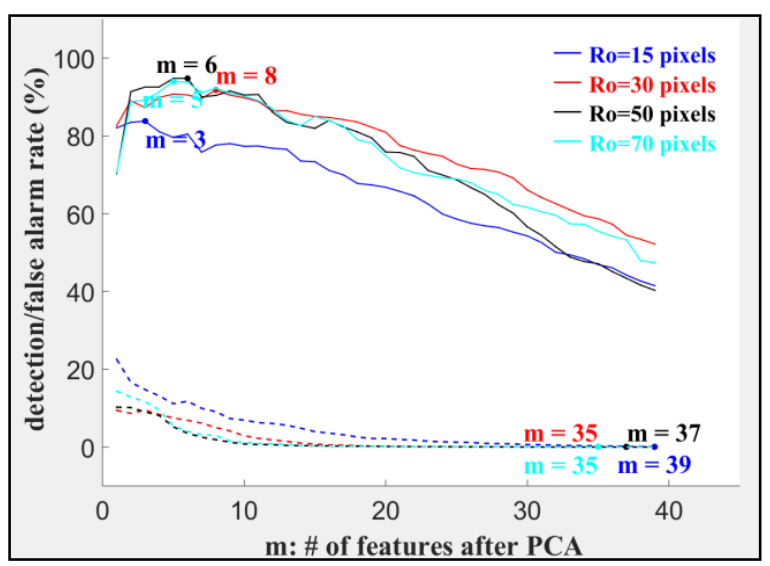

Figure 78 Detection/False Alarm Rates vs. KLT feature sizes after smoothing the classification results with $3 \%$ training set

Secondly, $\boldsymbol{R}_{\boldsymbol{o}}$ size is fixed to 50 pixels that is optimum $\boldsymbol{R}_{\boldsymbol{o}}$ size and sample-point number is changed to estimate optimum sample-point number. The detection results, false alarm rates, FP numbers are calculated and entire solutions are given in Table 20. The threehour data set is used during this calculation. The simulation results showed that sunset and sunrise times could be used instead of nighttime image cube. The simulation results contribution to the proposed method is given in section 8.3.

Optimum solutions are taken when the sample-point number selected as 24. Thus, optimum spatial filter is calculated as 50 pixels outer diameter with 24 sample-point number. 
Table 20 Sample-point numbers' influence on detection results

\begin{tabular}{|l|c|c|c|c|}
\hline \multirow{2}{*}{ Classifier } & $\begin{array}{c}\text { Sample } \\
\text { point } \\
\text { number }\end{array}$ & $\begin{array}{c}\text { Max. detection } \\
\text { rate (\%) }\end{array}$ & $\begin{array}{c}\text { False alarm rates } \\
(\%)\end{array}$ & FP \\
\hline \multirow{4}{*}{ QDA } & 12 & 92 & 1.89 & 4 \\
\cline { 2 - 5 } & $\mathbf{2 4}$ & $\mathbf{9 0}$ & $\mathbf{0 . 7 7}$ & - \\
\cline { 2 - 5 } & 48 & 82 & 0.34 & - \\
\cline { 2 - 5 } & 96 & 84 & 0.48 & - \\
\hline
\end{tabular}

\subsubsection{Performance Comparisons of the Daytime and Nighttime Image Cubes}

50-pixel $R_{o}$ spatial filter was applied to the daytime and nighttime image cubes, and $3 \%$ of classes were used as the training set. During application of the proposed method QDA algorithm was used. The results of detection rates and false alarm rates of the two data sets are given in Table 21 for comparison.

Table 21 Detection and false alarm rates with respect to imaging time of the day

\begin{tabular}{|l|c|c|}
\hline \multicolumn{1}{|c|}{ Data } & Max. detection rate (\%) & $\begin{array}{c}\text { False alarm rate (\%), } \\
\text { at max. detection rate point }\end{array}$ \\
\hline Nighttime & $\mathbf{9 5}$ & $\mathbf{5 . 2}$ \\
\hline Daytime & 82 & 25.1 \\
\hline
\end{tabular}

Broader results are plotted in Figure 79. In addition, the classification and detection results are given in figures from Figure 80 to Figure 83. The results are obtained using the optimum feature size that is 11 for QDA. 


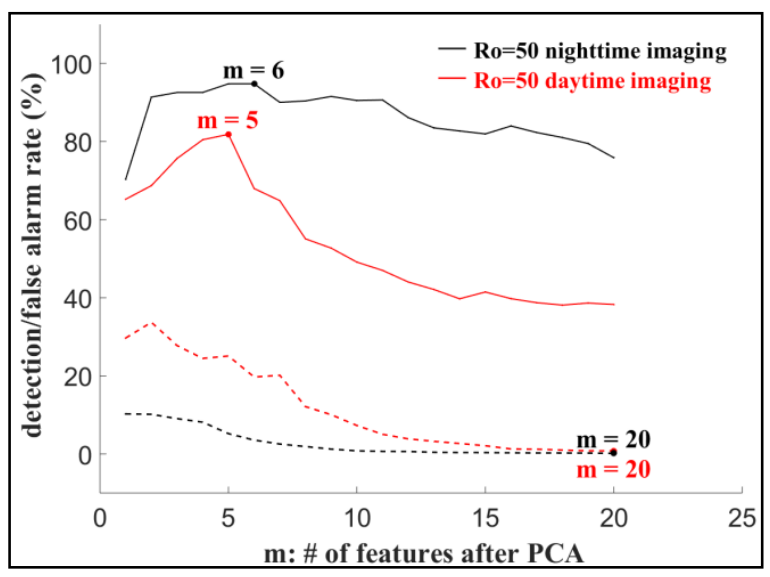

Figure 79 Detection/False Alarm Rates vs. KLT feature sizes after smoothing the classification results with respect to used data sets

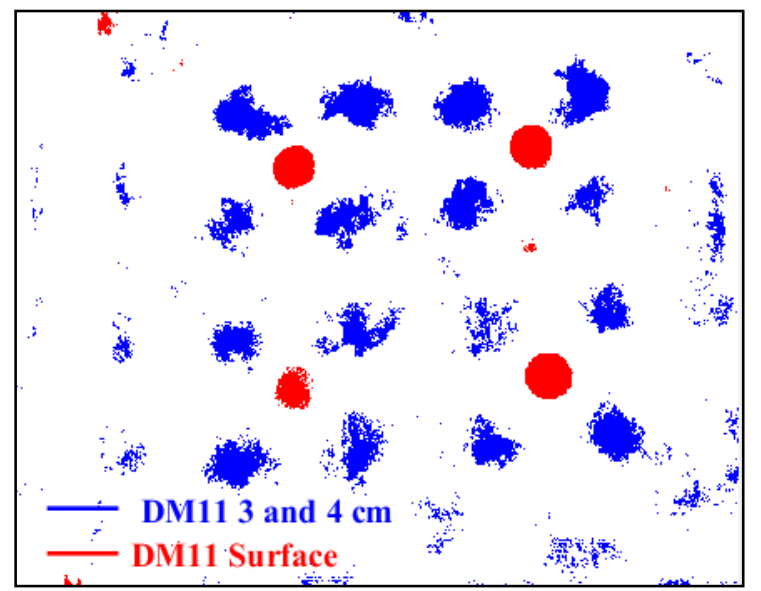

Figure 80 The classification result of nighttime image cube $(\mathrm{m}=11)$

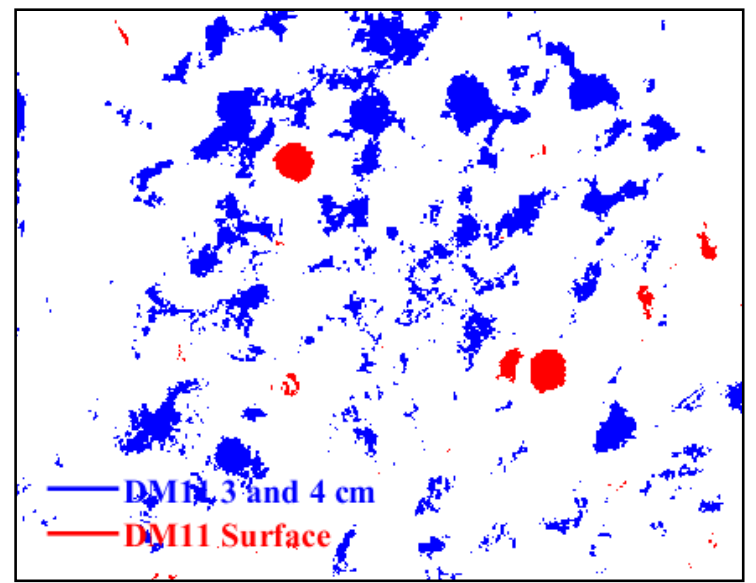

Figure 81 The classification result of daytime image cube $(\mathrm{m}=11)$ 


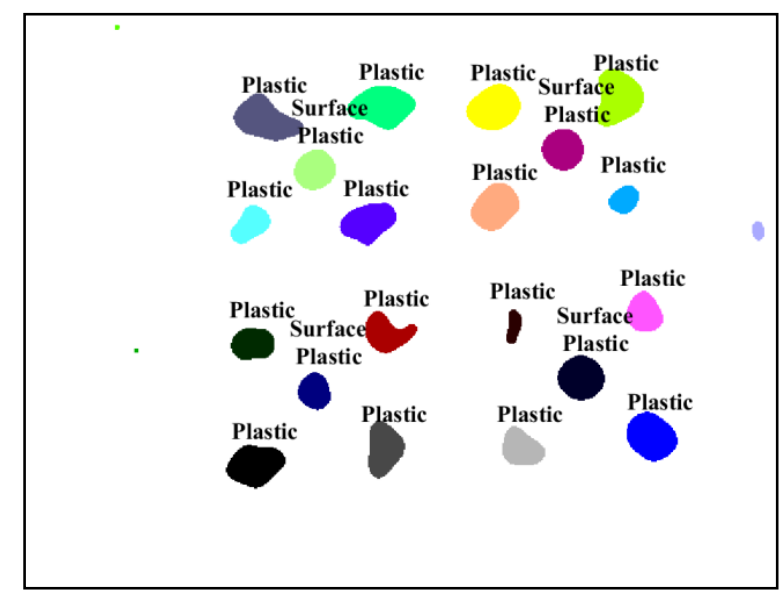

Figure 82 The detection result of nighttime image cube $(\mathrm{m}=11)$

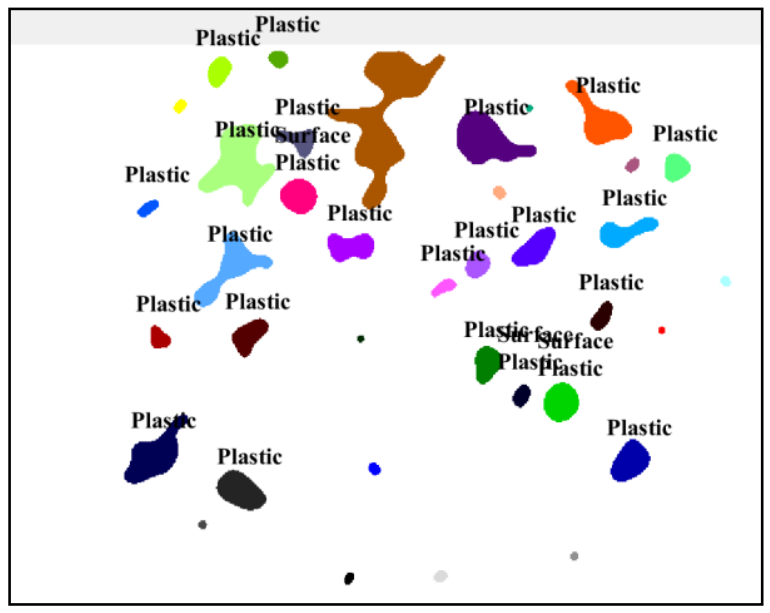

Figure 83 The detection result of daytime image cube $(m=11)$

It can be easily seen from Table 21 and Figure 80-Figure 83 that nighttime detection and false alarm rates are much better than daytime images as mentioned in Sections 7.2.1Error! Reference source not found. and 7.3.1.

\subsubsection{The Unfiltered (raw) and Filtered Image Cubes Performance Comparisons}

The unfiltered and filtered nighttime image cubes were used during comparison. 50pixel $R_{o}$ spatial filter was applied to the sunset and sunrise image cubes, and $3 \%$ of classes were used as the training set. During application of the proposed method QDA 
algorithm was used. The results of detection rates and false alarm rates of the two data sets are given in for comparison.

Table 22 Detection and false alarm rates comparisons of raw and filtered date

\begin{tabular}{|l|c|c|c|}
\hline Image Cube & Imaging period & $\begin{array}{c}\text { Max. detection rate } \\
(\%)\end{array}$ & $\begin{array}{c}\text { False alarm rate (\%), } \\
\text { at max. detection rate point }\end{array}$ \\
\hline Raw & \multirow{2}{*}{$9: 00 \mathrm{pm}-6: 45 \mathrm{am}$} & $\mathbf{9 1}$ & $\mathbf{1 3}$ \\
\cline { 3 - 4 } & Filtered & 95 & 5.2 \\
\hline
\end{tabular}

The classification and detection results are given in Figure 84 and Figure 85. The results of raw image cube are obtained using 12-feature size.

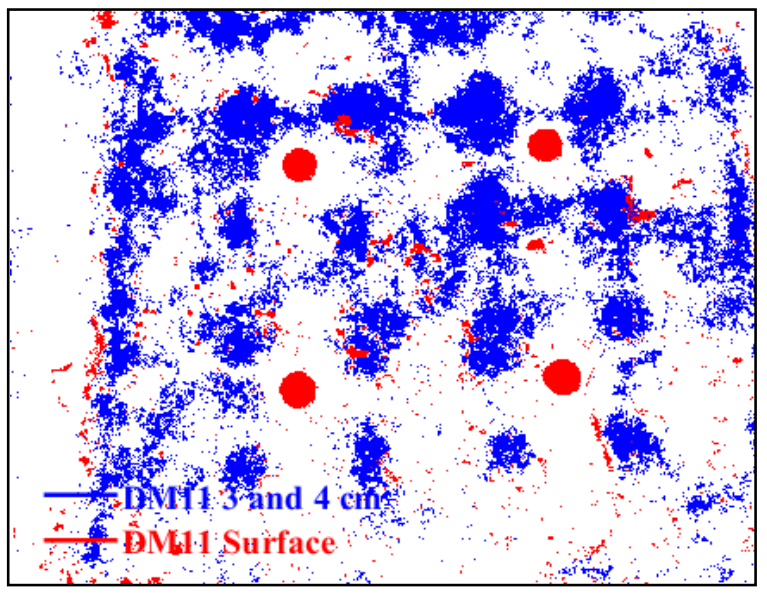

Figure 84 The classification result of nighttime raw image cube $(\mathrm{m}=12)$

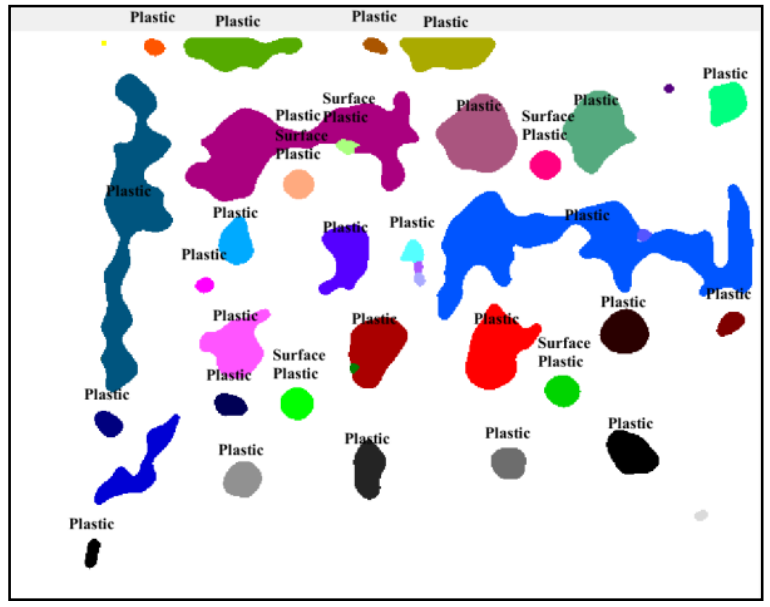

Figure 85 The detection result of nighttime raw image cube $(\mathrm{m}=12)$ 
Filtering image cube with presented spatial filter increases the detection rates and decreases the false alarm rates. The results can be seen in Table 22. In addition, same result can be inferred visually when comparing Figure 80 - Figure 84 and Figure 82 Figure 85. 


\section{CHAPTER 8}

\section{SIMULATION RESULTS AND DISCUSSIONS}

\subsection{M48, and DM11 PLs Simulation Results with the First Test Area's Parameters}

The M48 PLs were buried at $1.2 \mathrm{~cm}$ and $3.8 \mathrm{~cm}$ and DM11 plastic PLs were buried two and three $\mathrm{cm}$ (Figure 13) in the first minefield. However, in this section one $\mathrm{cm}$, three $\mathrm{cm}$, and seven $\mathrm{cm}$ burial depth results are given in order to see soil depth influence on temperature differences between the soil surface above a PL and bare soil.

\subsubsection{M48 Metal PL Simulation Results}

When the burial depth is selected as one $\mathrm{cm}$, diurnal temperature change is formed as in Figure 86 that reflects temperature change along the $80 \mathrm{~cm}$ soil surface during the diurnal cycle. The PL is at the center of the cross-section and it can be easily seen that there is a large temperature difference between PL encountered soil surface and the soil surface itself. Even the PL diameter can be predicted from Figure 86.

Figure 88 was taken at 6 a.m. on 9 September 2015 by the FLIR camera. The $1.8 \mathrm{~cm}$ deep M48 PL is seen on the very top-right corner of the image. If the pixel resolution is known, the PL diameter can be predicted from the image.

Change of the PL's soil-top surface and the soil-surface temperature difference are given in Figure 89. The black line shows temperature change between PL encountered soil surface and the soil surface, red line reflects temperature differences between one $\mathrm{cm}$ deep soil temperature and soil surface temperature. Vertical blue lines show sunset and sunrise times, and vertical fine red lines show the maximum difference times between 
PL encountered soil surface, and the soil surface. As can be seen from Figure 86 and Figure 89, the metal PL is colder is during the daytime and the maximum difference $\left(6.35^{\circ}\right)$ emerges at two pm, and the nighttime PL is hotter and the maximum positive difference $\left(3.69^{\circ}\right)$ occurs at 5:15 am. The temperature variations though the soil crosssection is given in Figure 87 at the maxima times.

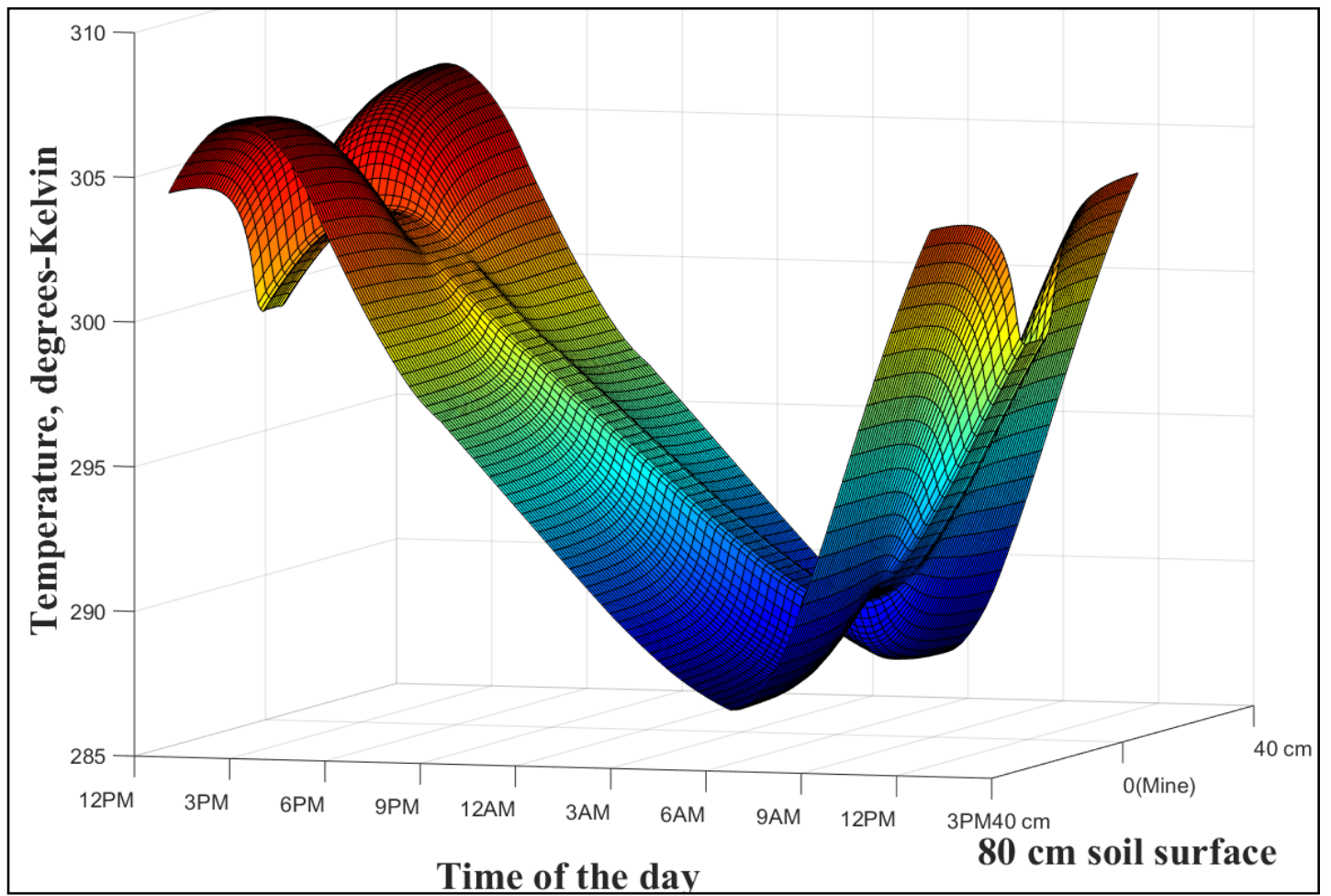

Figure 86 3-D temperature distribution on the soil surface during diurnal cycle $($ depth $=1 \mathrm{~cm})$

Figure 90 reflects the cross section of temperature distribution along the soil surface when these differences are the largest. The PL surface encountered soil surface pixels are also distinguished in this figure.

It can be understood from Figure 89 that the difference of one cm depth soil temperatures and soil surface temperatures are quite small. However, the gradient magnitude during daytime is larger than nighttime's. 


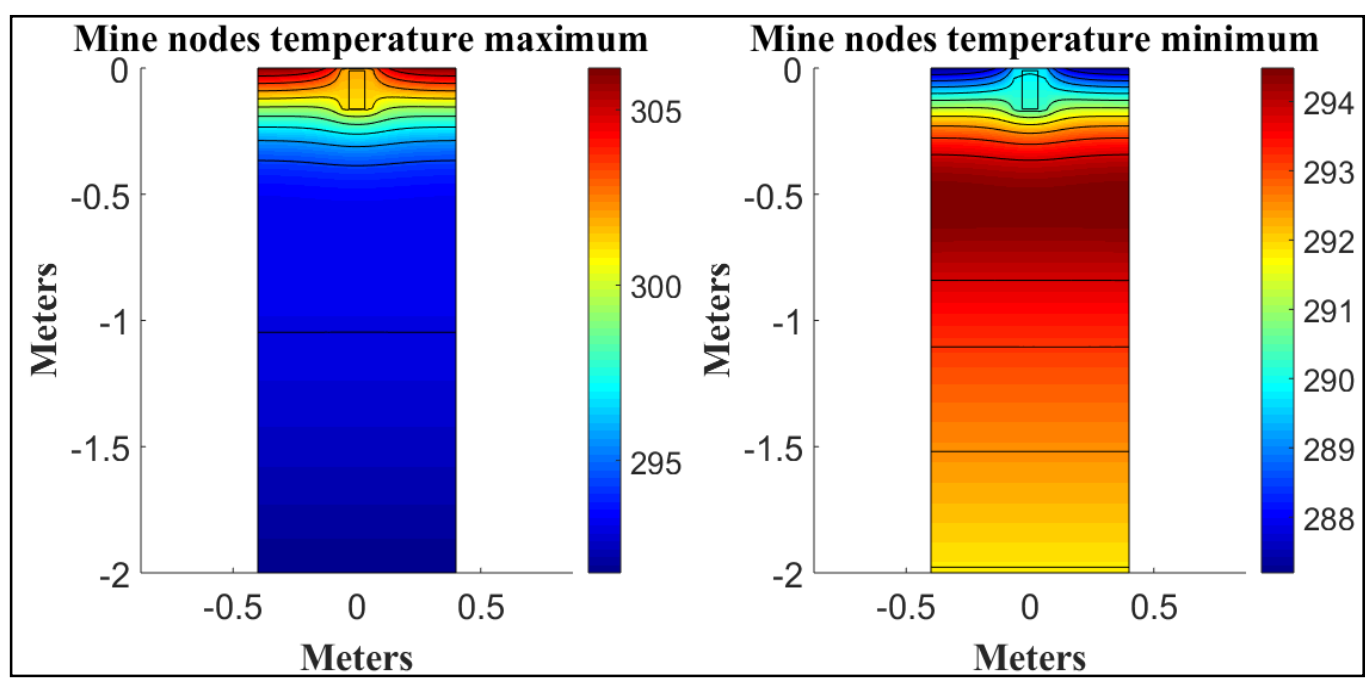

Figure 87 Cross-section of temperature distribution when the PL encountered soil surface, and soil surface's temperature differences are maximum

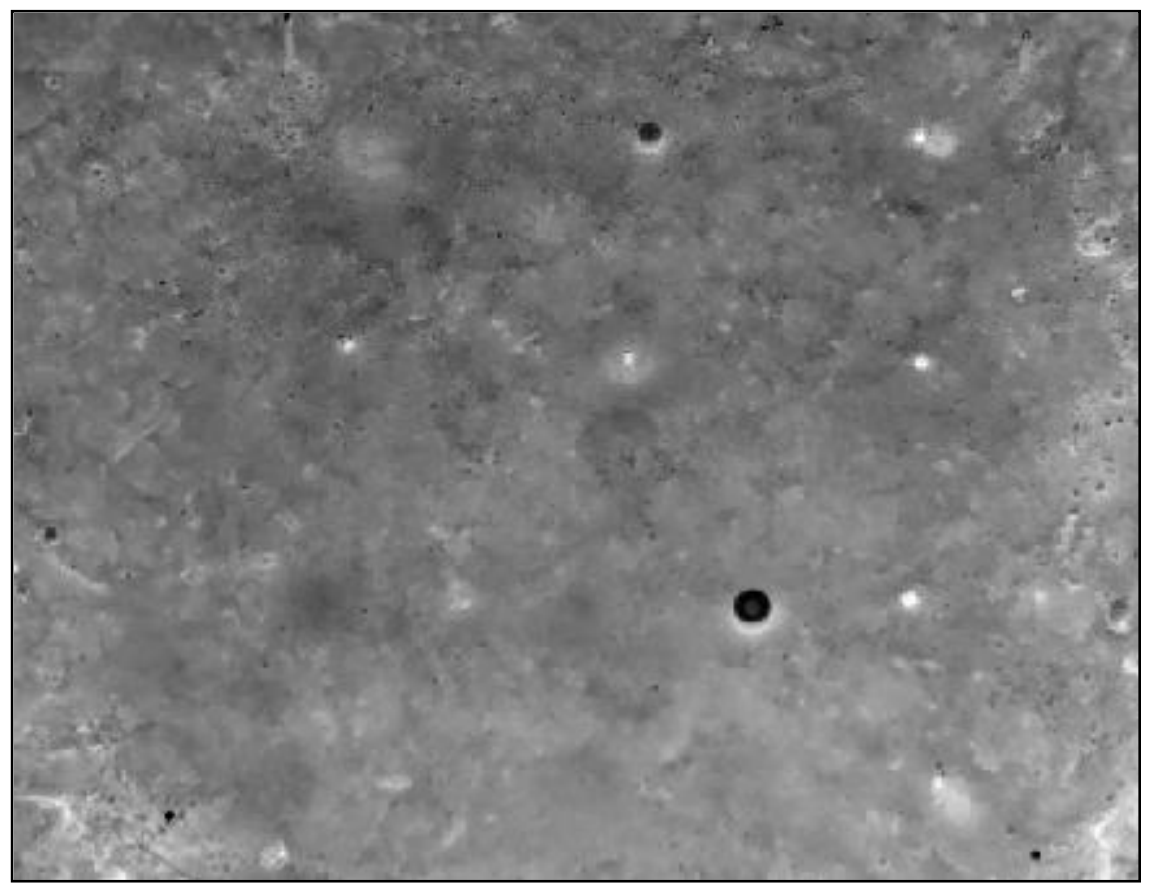

Figure 88 The image captured at 6 am with FLIR T 650 SC on 9 September 2015 


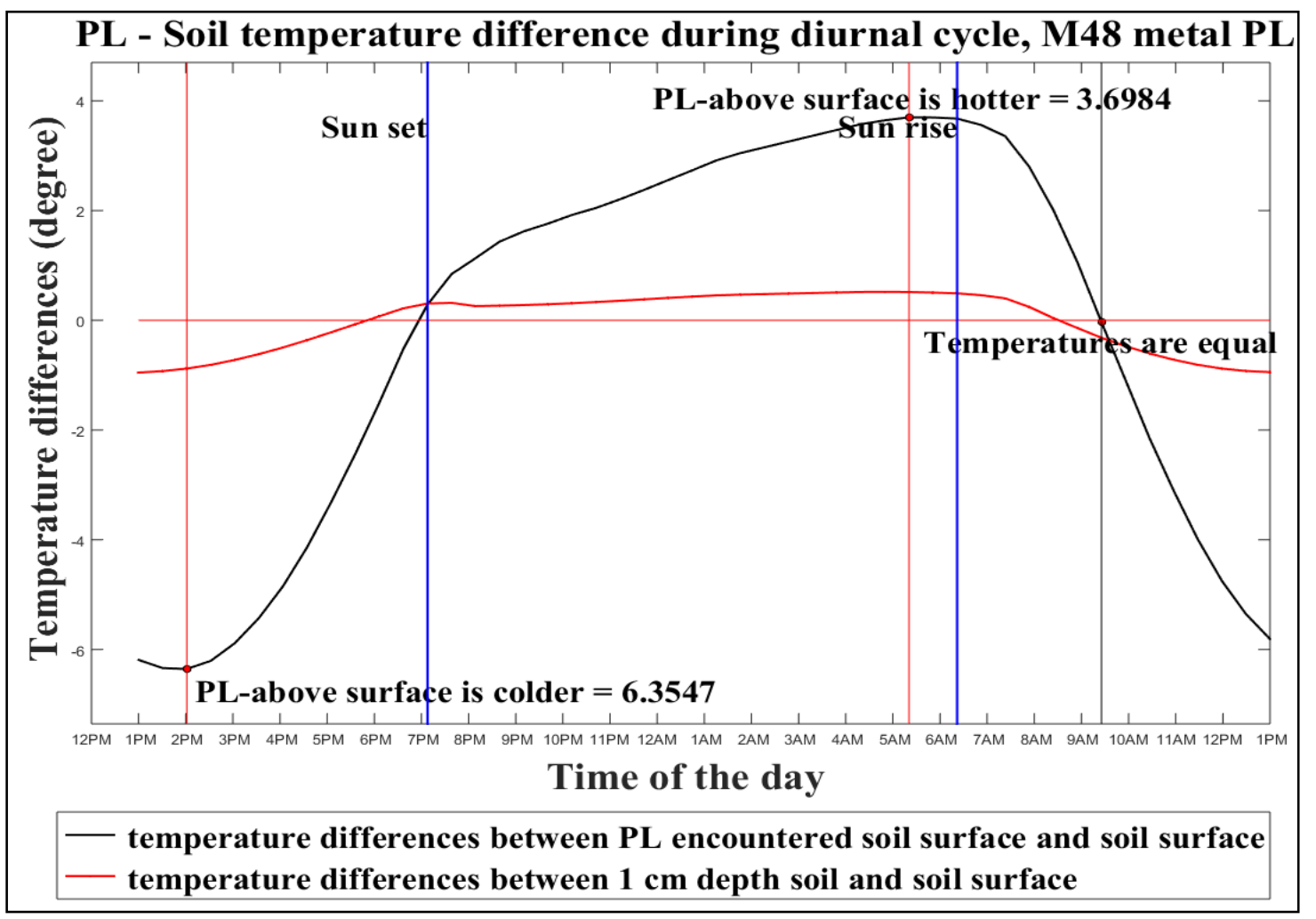

Figure 89 Soil surface temperature and PL encountered soil surface/deep soil temperature difference during diurnal cycle $($ depth $=1 \mathrm{~cm})$

When the metal PL is buried at three $\mathrm{cm}$ depth the magnitude of the maximum temperature difference gets smaller. The maximum differences are $4.98^{\circ}, 3.07^{\circ}$, and they occur at 2:00 pm, and at 5:45 am respectively. The entire temperature distributions along the cross-section of the soil at these times can be seen in Figure 92. However, the gradient of deep soil temperature chance gets larger during the daytime (Figure 91, Figure 93). 


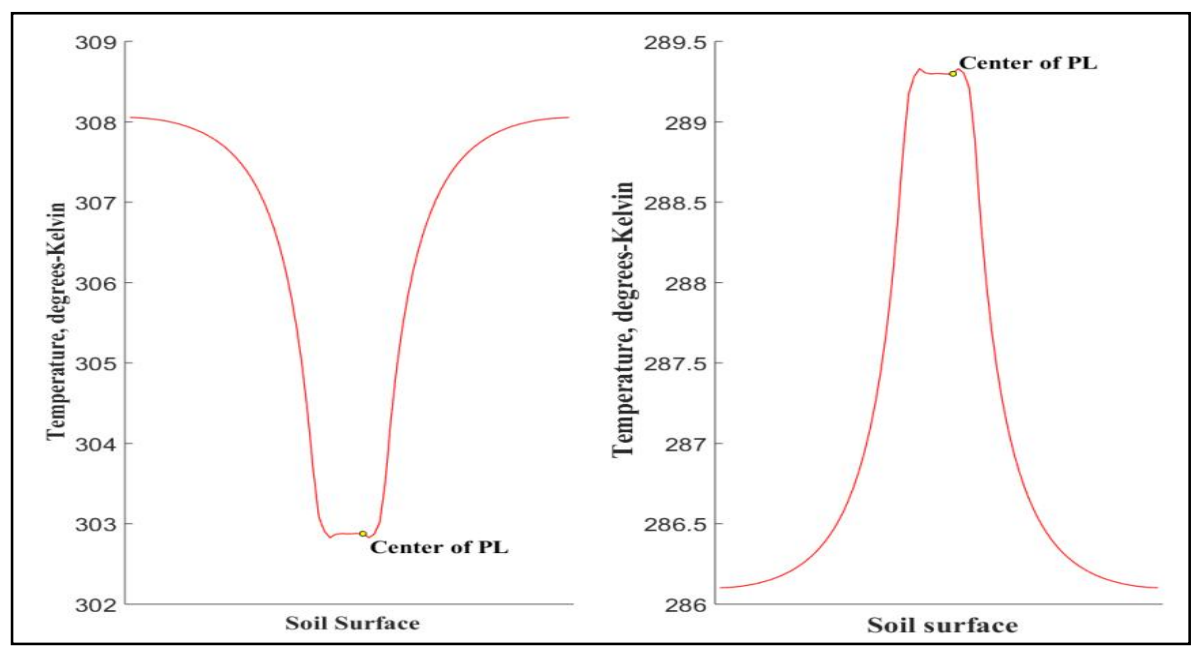

Figure 90 The soil surface temperature distribution $($ depth $=1 \mathrm{~cm})$

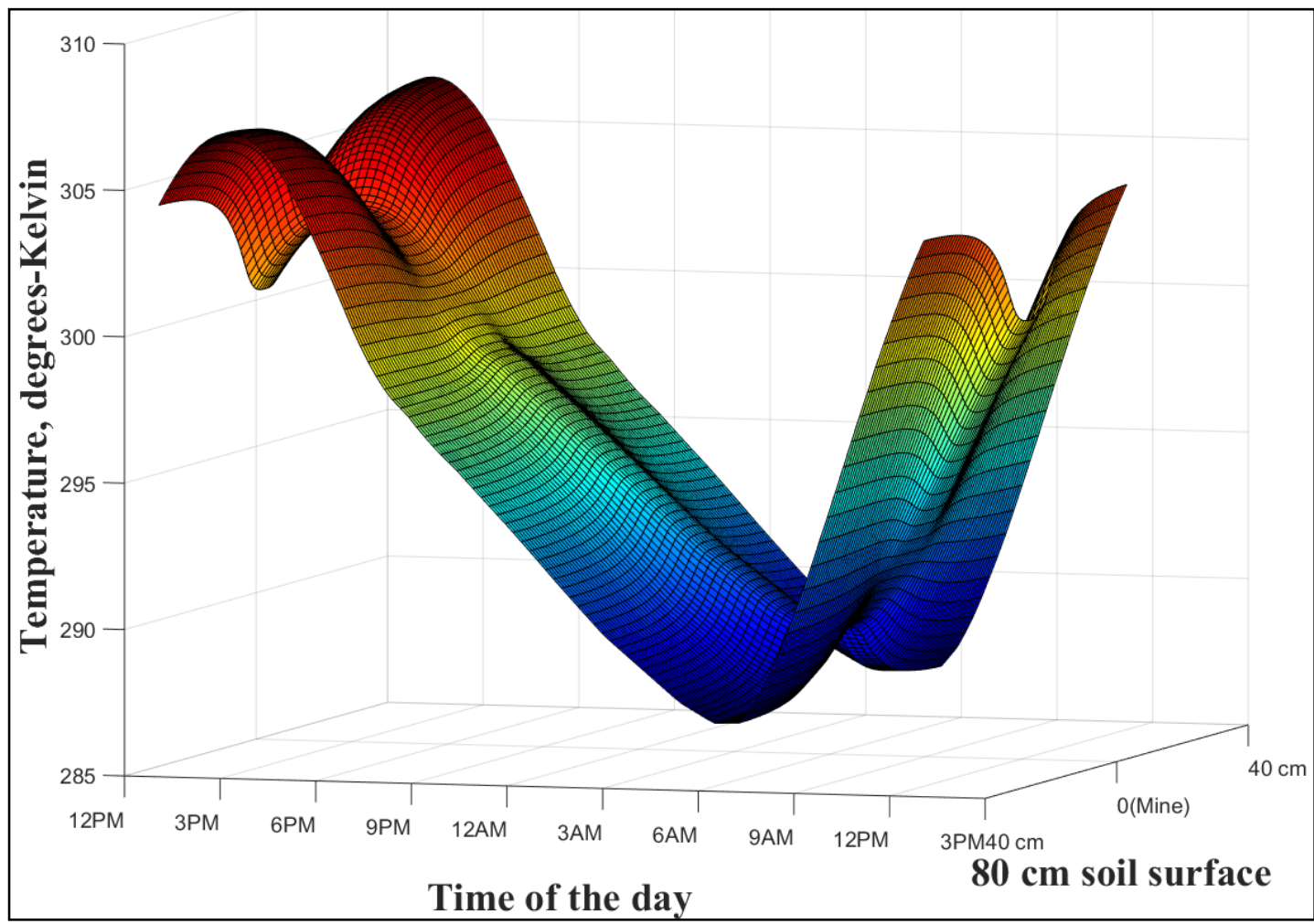

Figure 91 3-D temperature distribution on the soil surface during diurnal cycle (depth $=$ $3 \mathrm{~cm}$ ) 


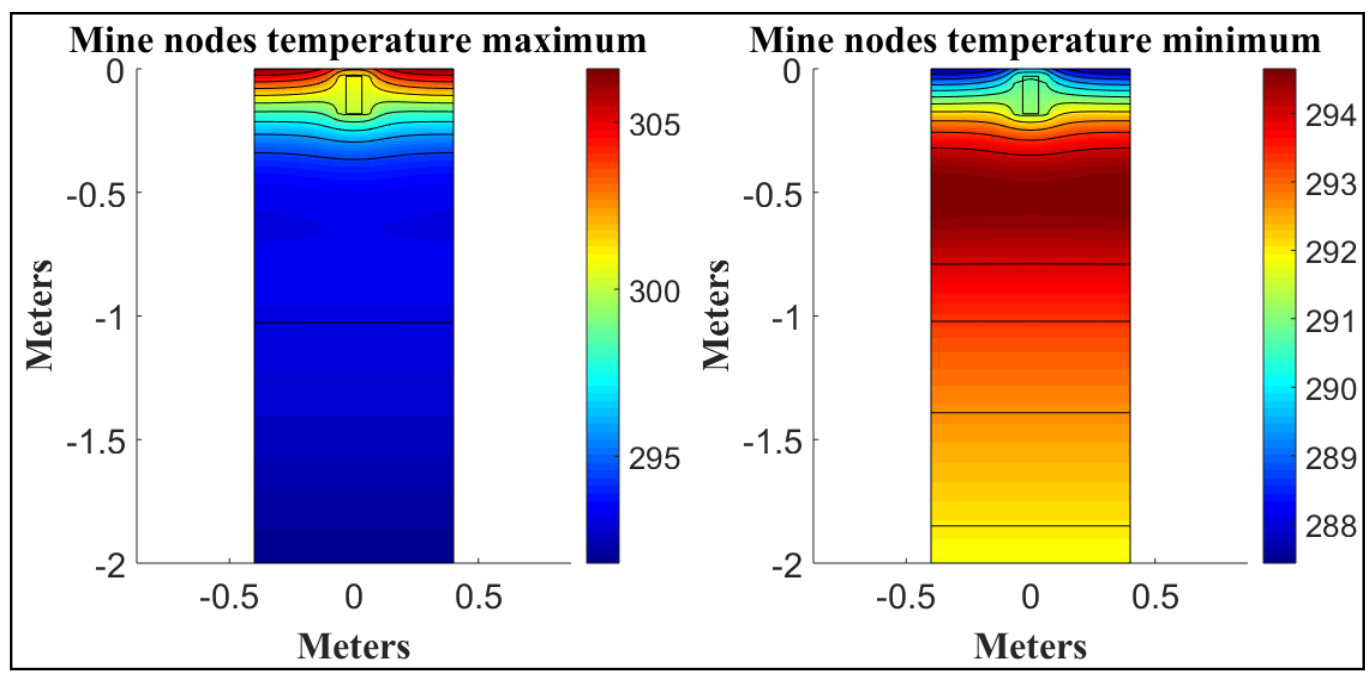

Figure 92 Cross-section of temperature distribution when the PL encountered soil surface, and soil surface's temperature differences are maximum

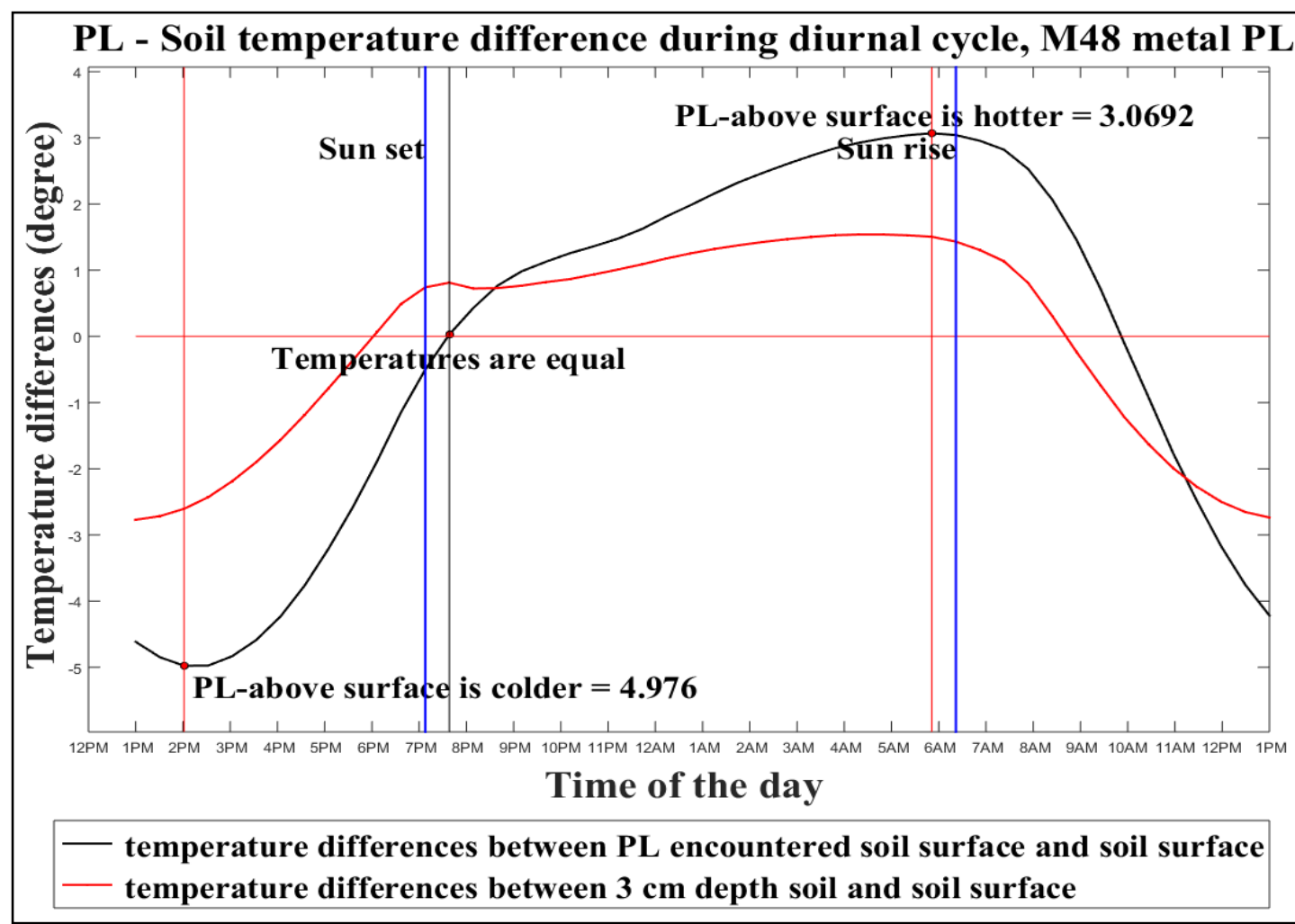

Figure 93 Soil surface temperature and PL encountered soil surface/deep soil temperature difference during diurnal cycle $($ depth $=3 \mathrm{~cm})$ 
Contrary to one $\mathrm{cm}$ depth, the soil-surface temperature distribution gets curved shape where there is PL encountered soil surface (Figure 94). However, the PL still can be seen with naked eye. The M2 PL, whose diameter is identical with M48, is located at the very right bottom corner of the Figure 88, can be distinguished.

When the burial depth increased to seven $\mathrm{cm}$, the temperature differences between PL encountered soil surface and soil surface gets smaller. Maximum differences are $2.82^{\circ}$ and $1.78^{\circ}$. The differences take place at 3:30 pm, and at 6:45 am, respectively (Figure 96). The temperature distribution along the cross-section of soil at these times is given in Figure 97.

When the soil depth increases, the temperature differences between the soil surface and deep soil increase as well. The reason is that when going in deeper soil, temperature gets cooler with respect to the soil surface temperature until a specific depth. At this depth, soil temperature stays nearly stable. However, surface temperature does continuously chance. Especially during daytime, soil surface gets hotter after sunrise and the gradient gets larger as well, on the other hand during nighttime soil surface temperature relatively stays stable so that gradient becomes stable and relatively small too. The valleys' depths and hills' heights are getting smaller when the depth of PL increases. When Figure 95, Figure 94, Figure 91 and Figure 86 are compared, the change among them can be easily seen.

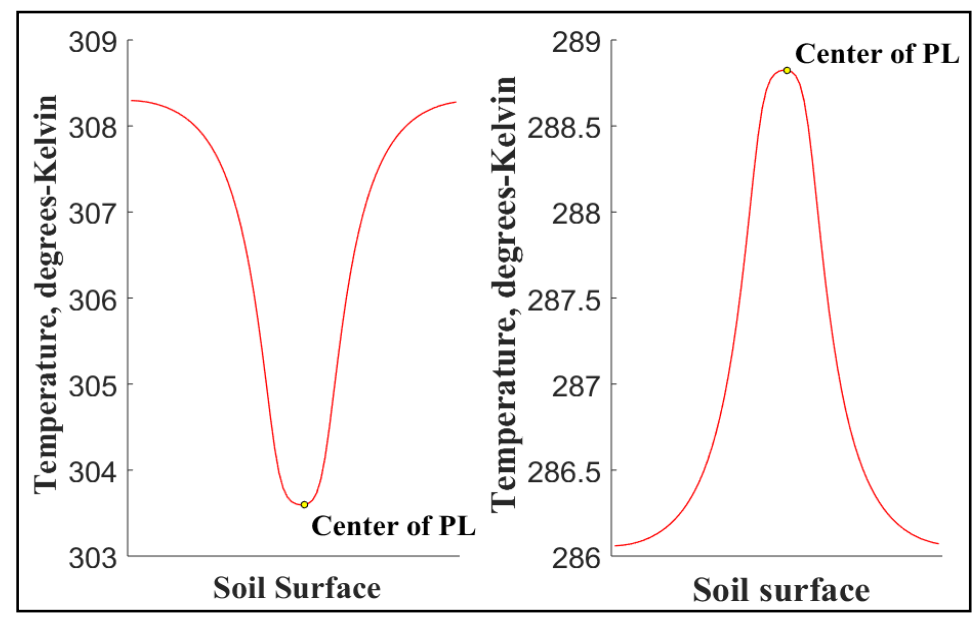

Figure 94 The soil surface temperature distribution $($ depth $=3 \mathrm{~cm})$ 


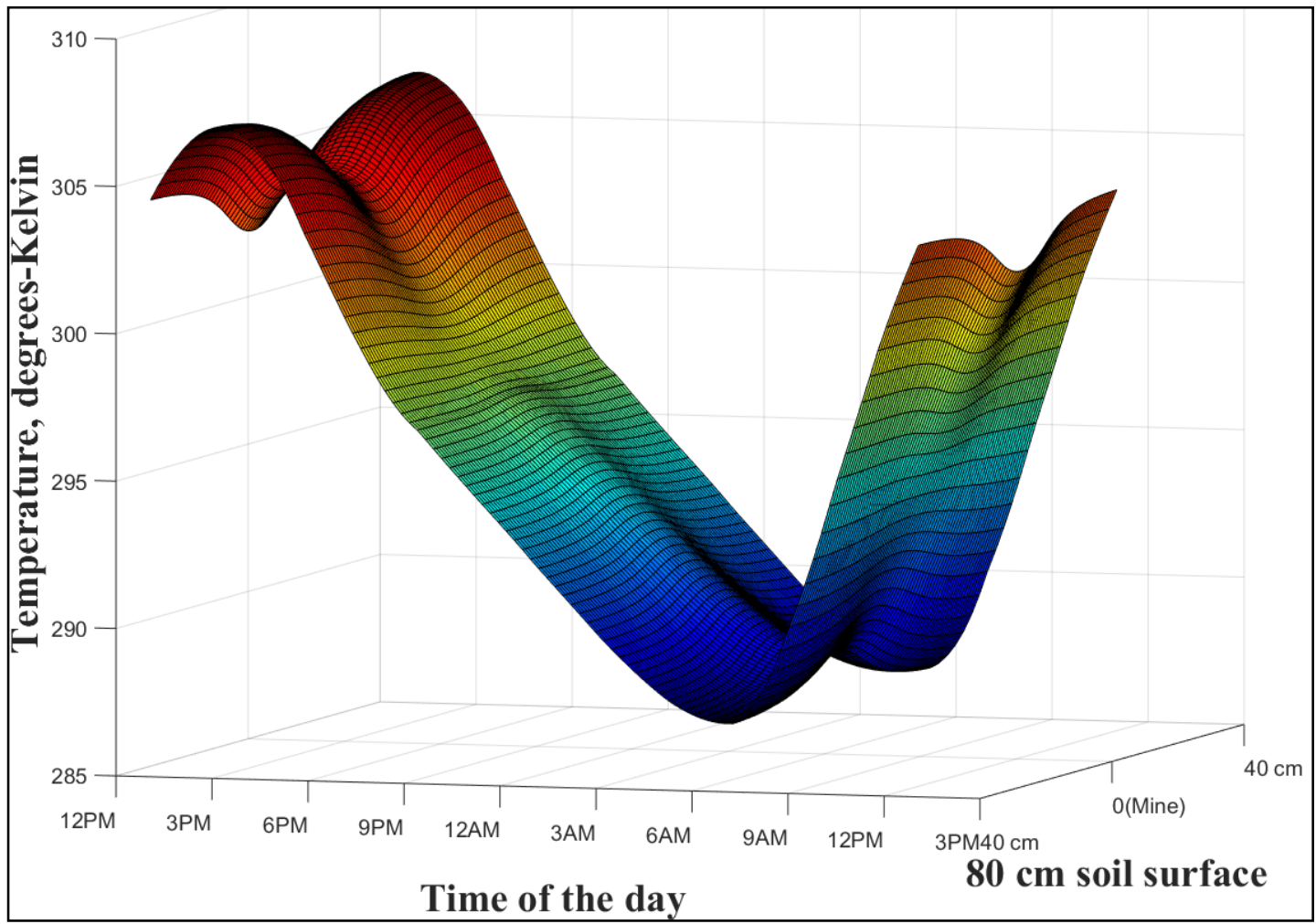

Figure $953-\mathrm{D}$ the temperature distribution on the soil surface during diurnal cycle $($ depth $=7 \mathrm{~cm})$

After three cm depth, metal PLs can be detected with neither naked eye, nor proposed method from thermal images without applying presented spatial filter. Proposed method using spatially filtered data set found $6.8 \mathrm{~cm}$ depth metal PLs. This shows that $1.78^{\circ}$ temperature difference is enough to detect metal PL that is buried seven cm depth.

\subsubsection{DM11 Plastic PL Simulation Results}

Contrary to metal PL, plastic PL encountered soil surface is hotter than soil surface during daytime and cooler during nighttime. 3-D the temperature distribution on the soil surface during diurnal cycle is given in Figure 98 when the PL is buried one cm depth. The PL location is obvious. 


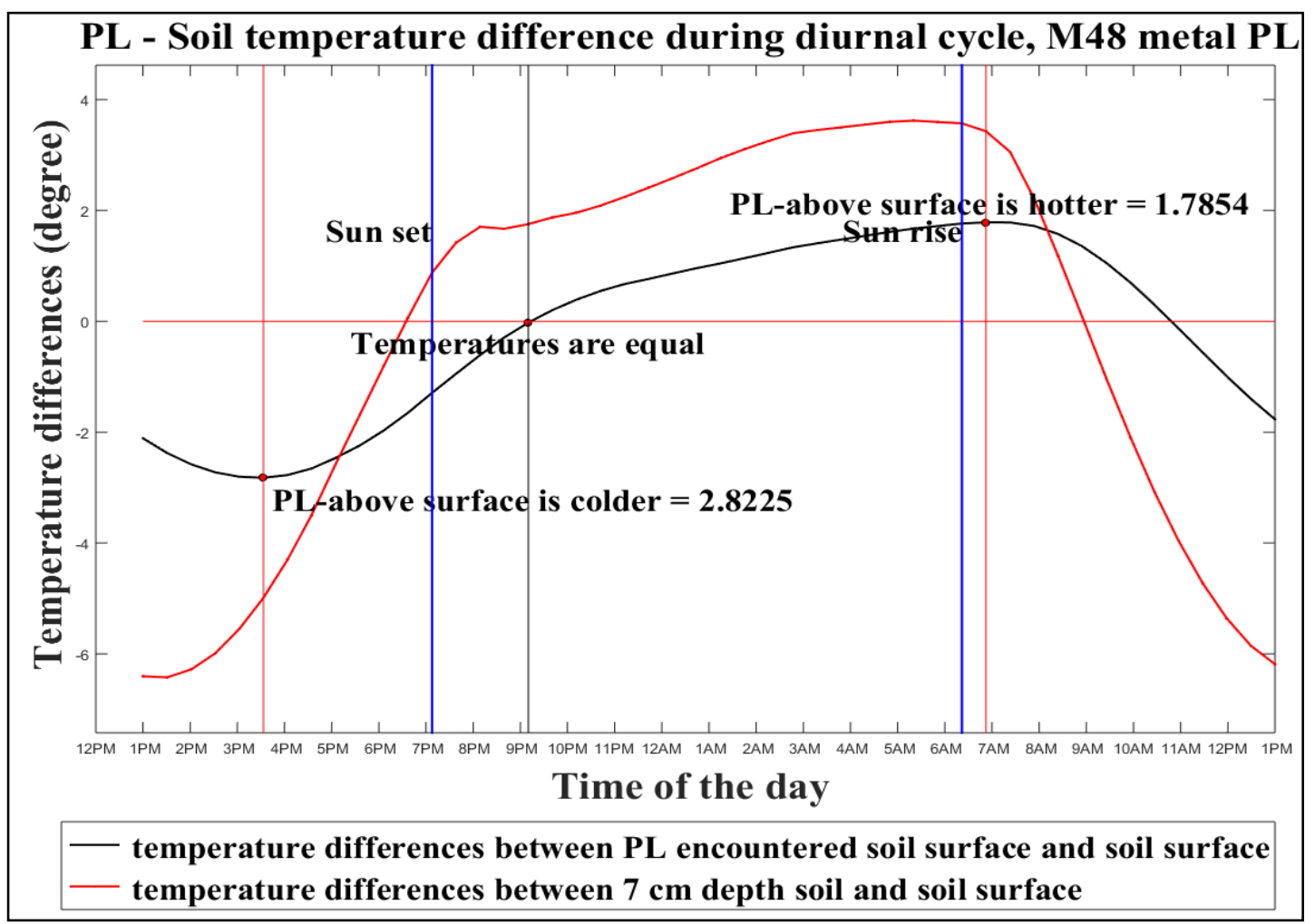

Figure 96 Soil surface temperature and PL encountered soil surface/deep soil temperature difference during diurnal cycle $($ depth $=7 \mathrm{~cm})$

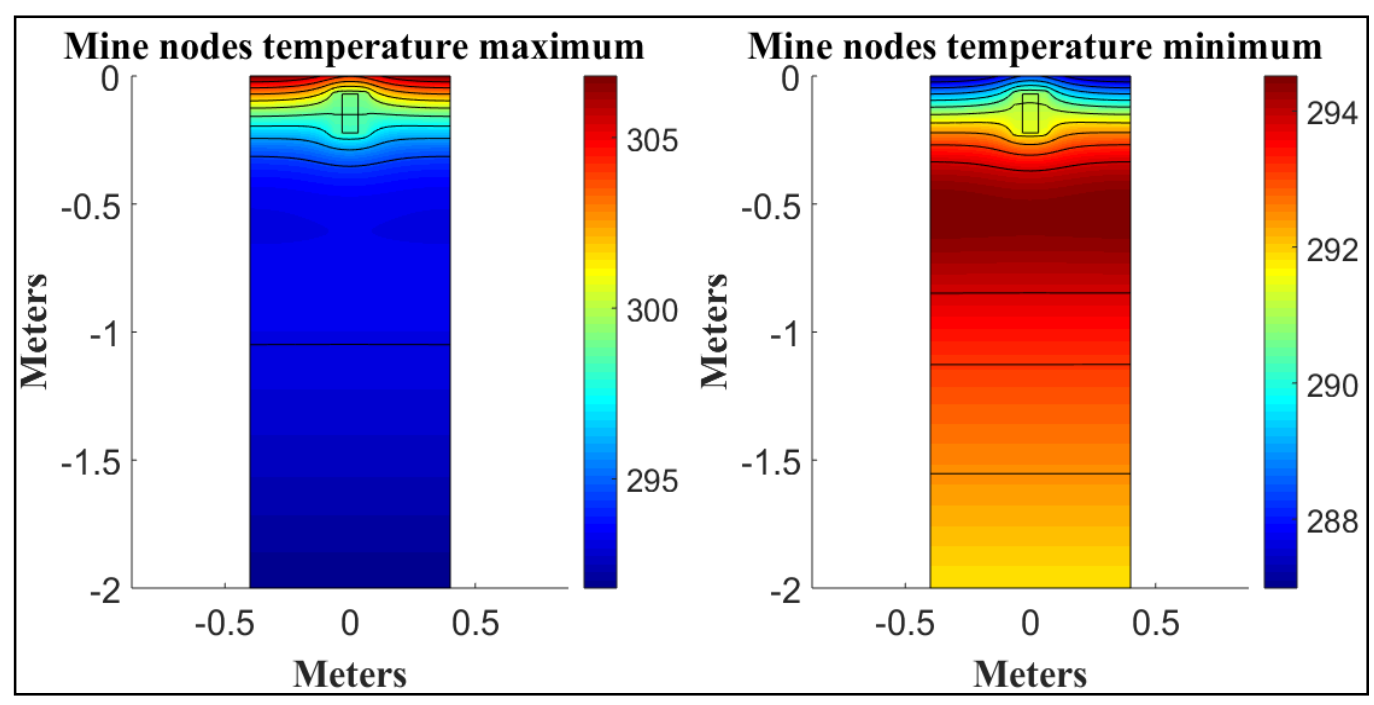

Figure 97 Cross-section of temperature distribution when the PL encountered soil surface, and soil surface's temperature differences are maximum 


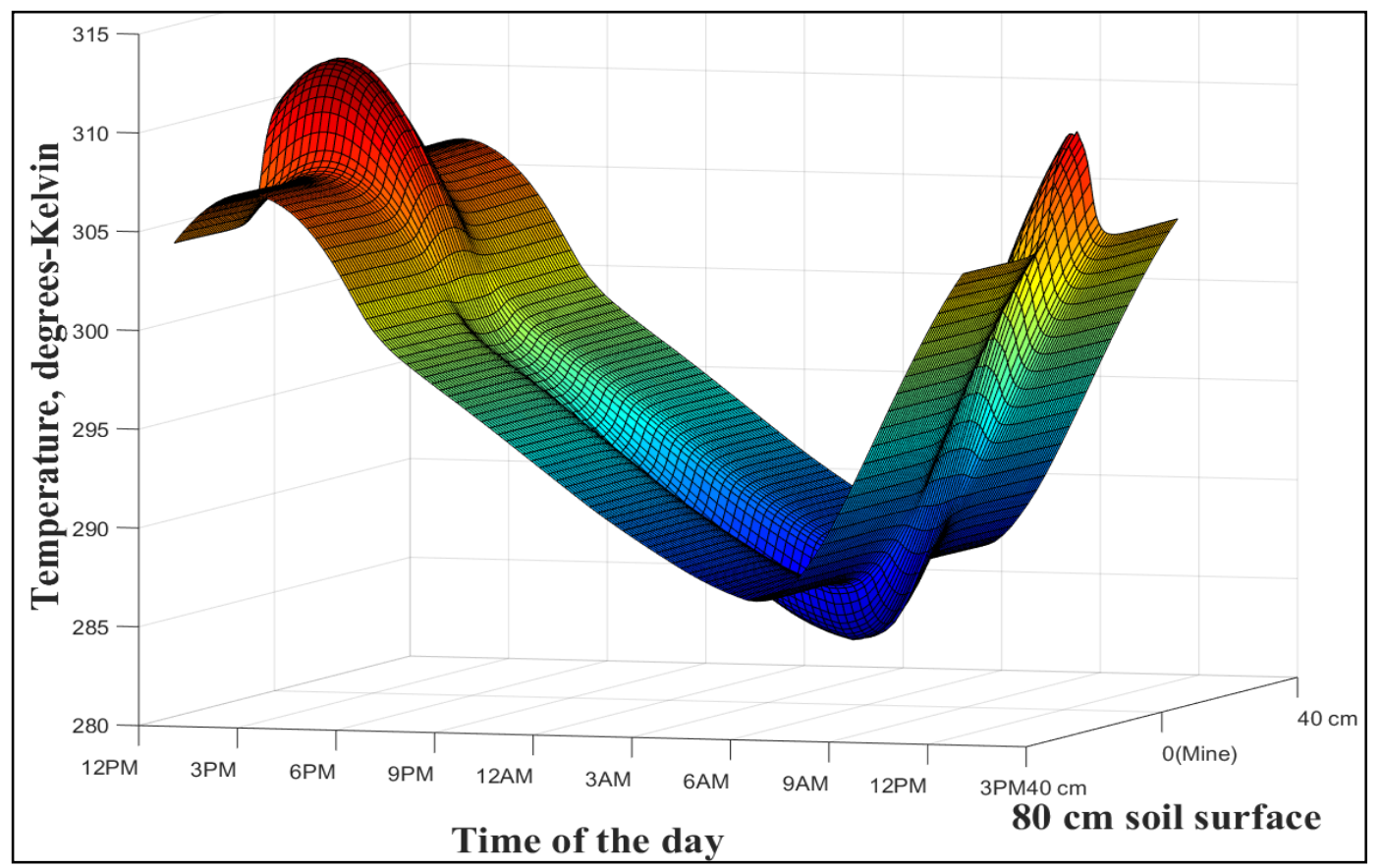

Figure 98 3-D the temperature distribution on the soil surface during diurnal cycle $($ depth $=1 \mathrm{~cm})$

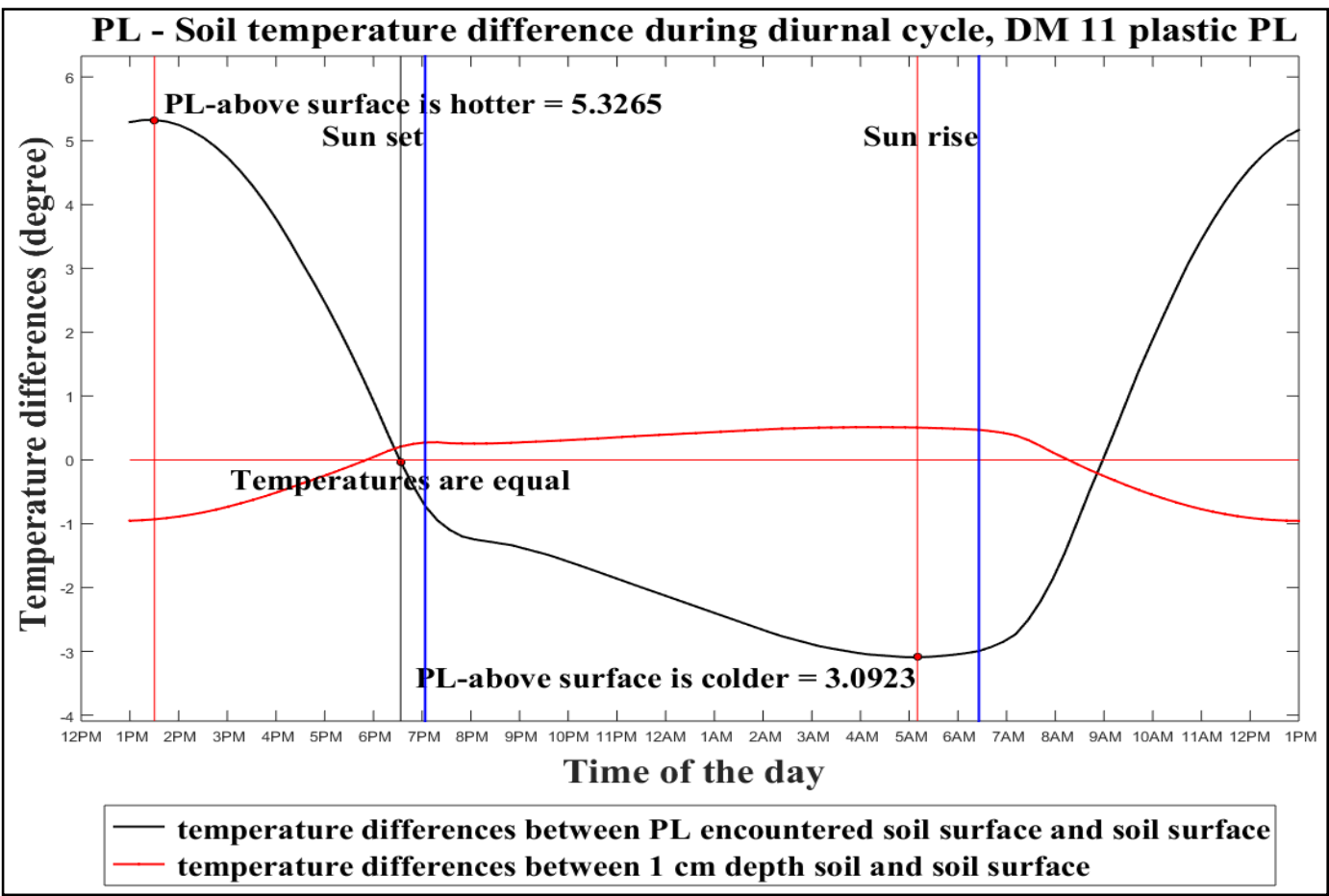

Figure 99 Soil surface temperature and PL encountered soil surface/deep soil temperature difference during diurnal cycle $($ depth $=1 \mathrm{~cm})$ 
The differences of the plastic PL encountered soil surface temperatures and soil surface temperatures through the diurnal cycle are given in Figure 99. Maxima differences occur at 1:30 pm and 5:15 am, the values at these times are $5.32^{\circ}$ and $3.09^{\circ}$ respectively.

The soil surface temperature-distribution is plotted in Figure 100 at the maximal difference times, and the cross-section is given in Figure 101.

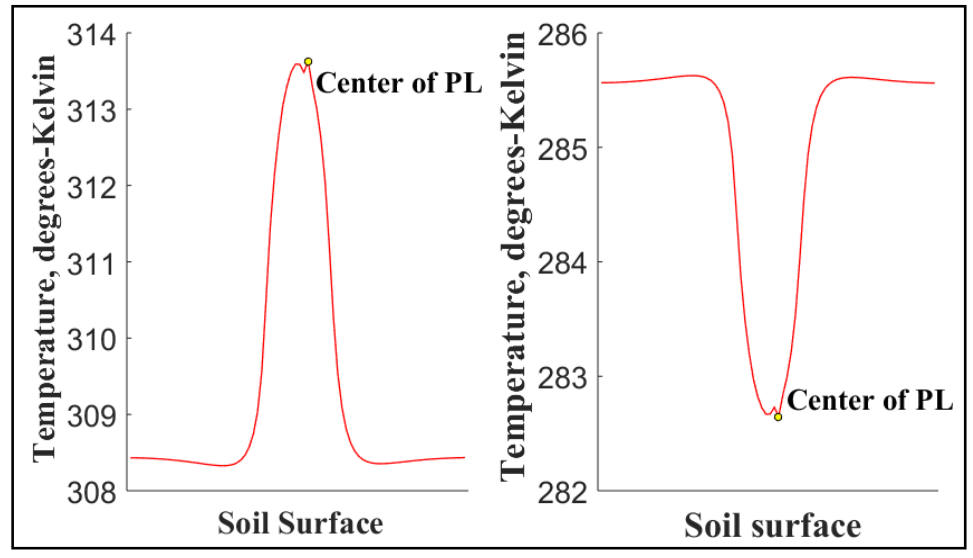

Figure 100 The soil surface temperature distribution $($ depth $=1 \mathrm{~cm})$

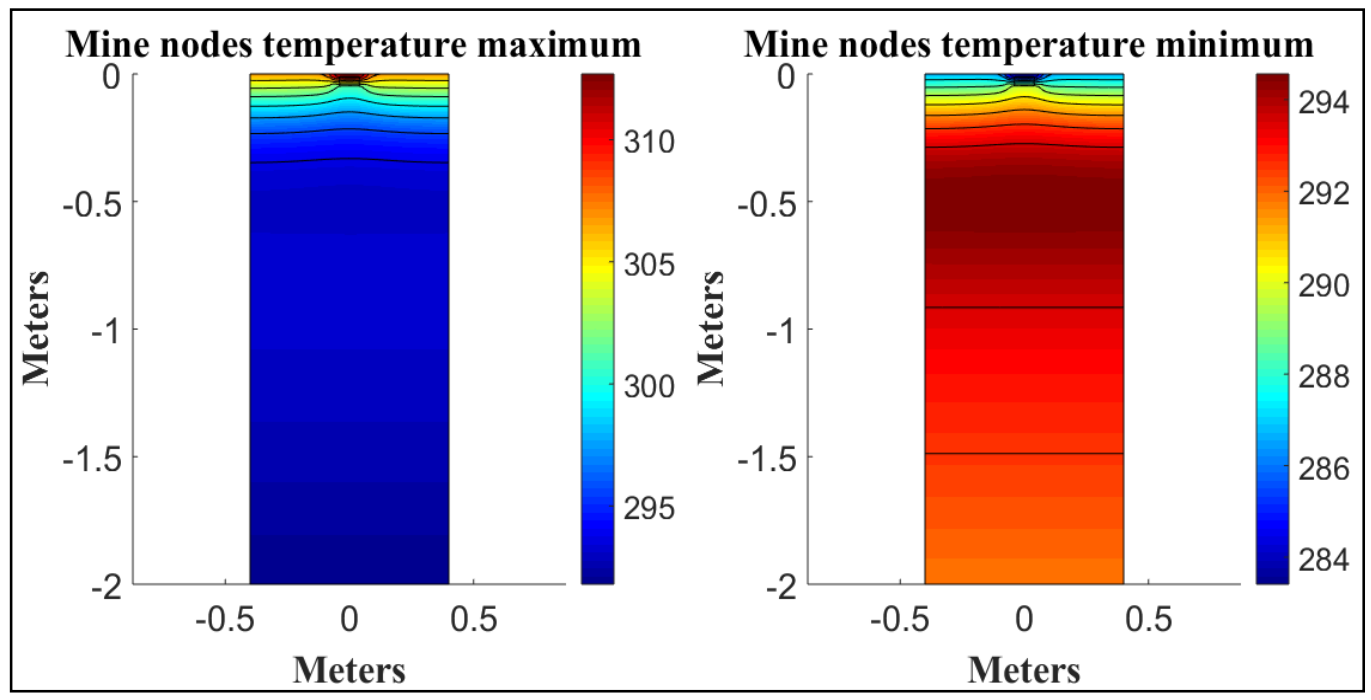

Figure 101 Cross-section of temperature distribution when the PL encountered soil surface, and soil surface's temperature differences are maximum 


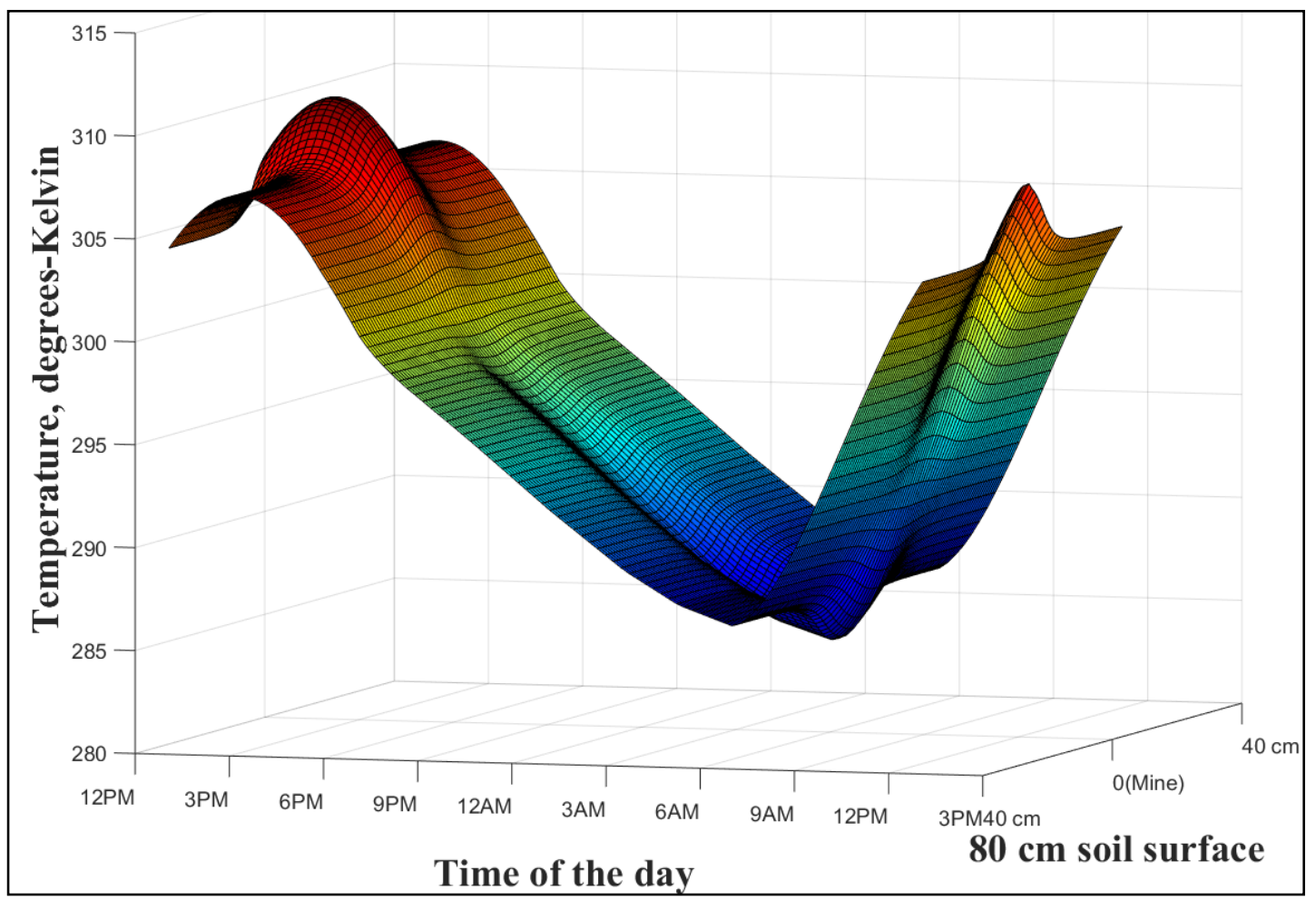

Figure 1023 -D the temperature distribution on the soil surface during diurnal cycle $($ depth $=3 \mathrm{~cm})$

In the event that buried depth is chosen, the maximum differences become $3.29^{\circ}, 1.96^{\circ}$ at 1:45 pm, and 5.15 am respectively. The 3-D temperature distribution is given in Figure 102, the temperature differences distribution between PL-encountered soil surface and soil surface is given in Figure 103, soil-surface temperature distribution, and temperature change along the soil cross-section at these times are given in Figure 104 and Figure 105, respectively.

Even though plastic landmines are not buried seven $\mathrm{cm}$ deep, the solutions are given in Figure 106, Figure 107, Figure 108 and Figure 109 for this depth in order to see the thermal distribution on the soil surface. The maximum differences take place at 3:15 pm, and 7:00 am. The maximum differences values are $1.52^{\circ}$, and $0.94^{\circ}$, respectively. 


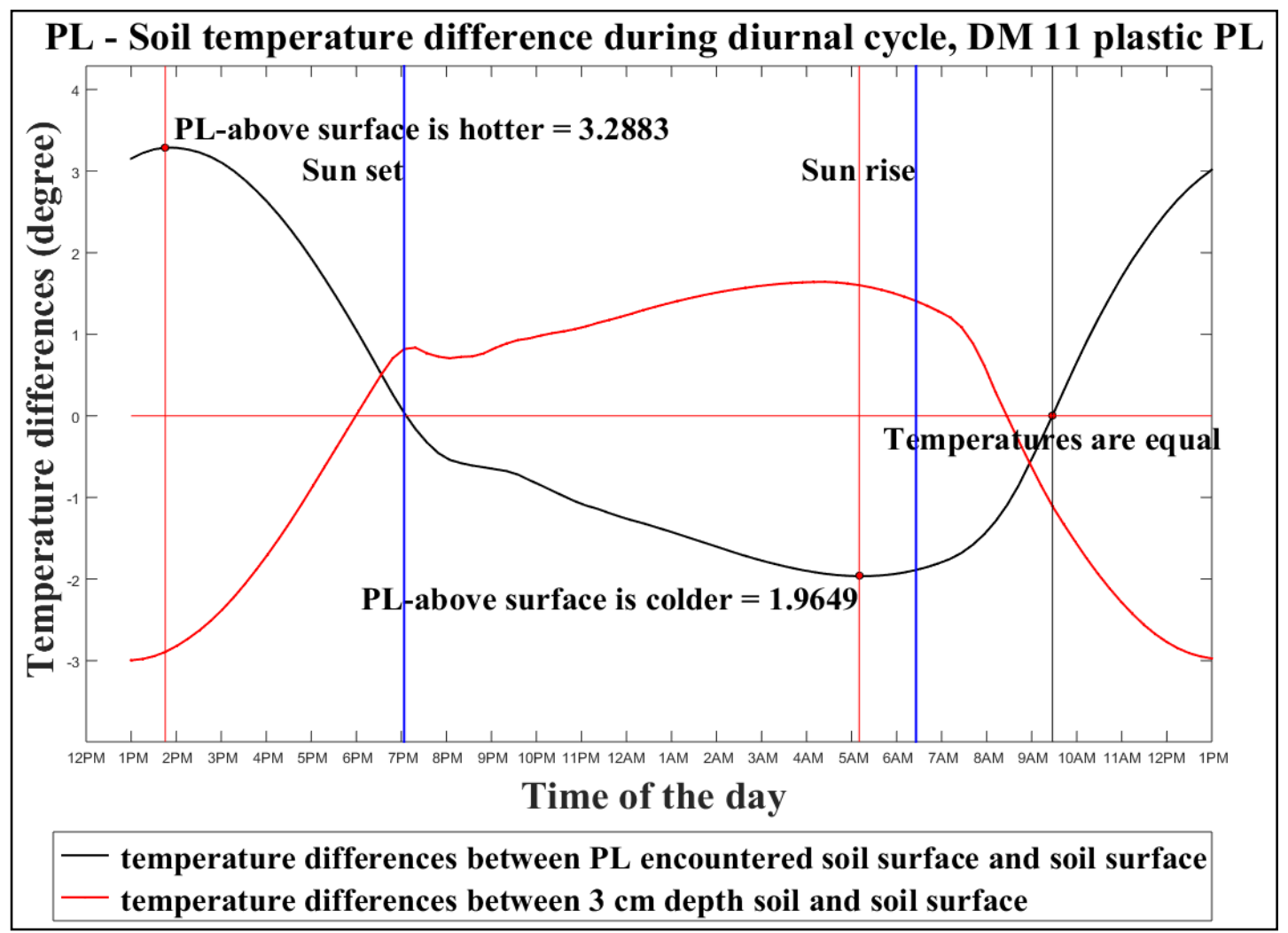

Figure 103 Soil surface temperature and PL encountered soil surface/deep soil temperature difference during diurnal cycle $($ depth $=3 \mathrm{~cm})$

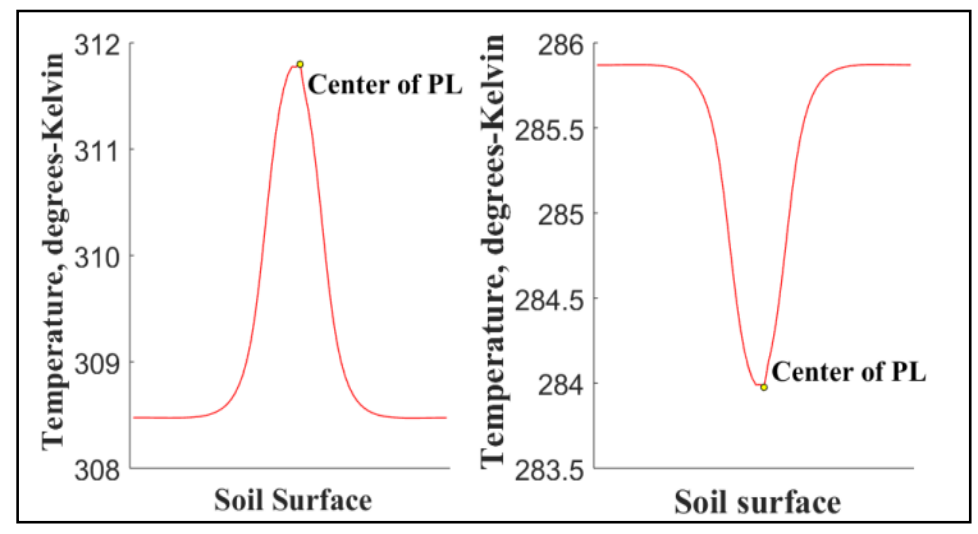

Figure 104 The soil surface temperature distribution $($ depth $=3 \mathrm{~cm})$ 


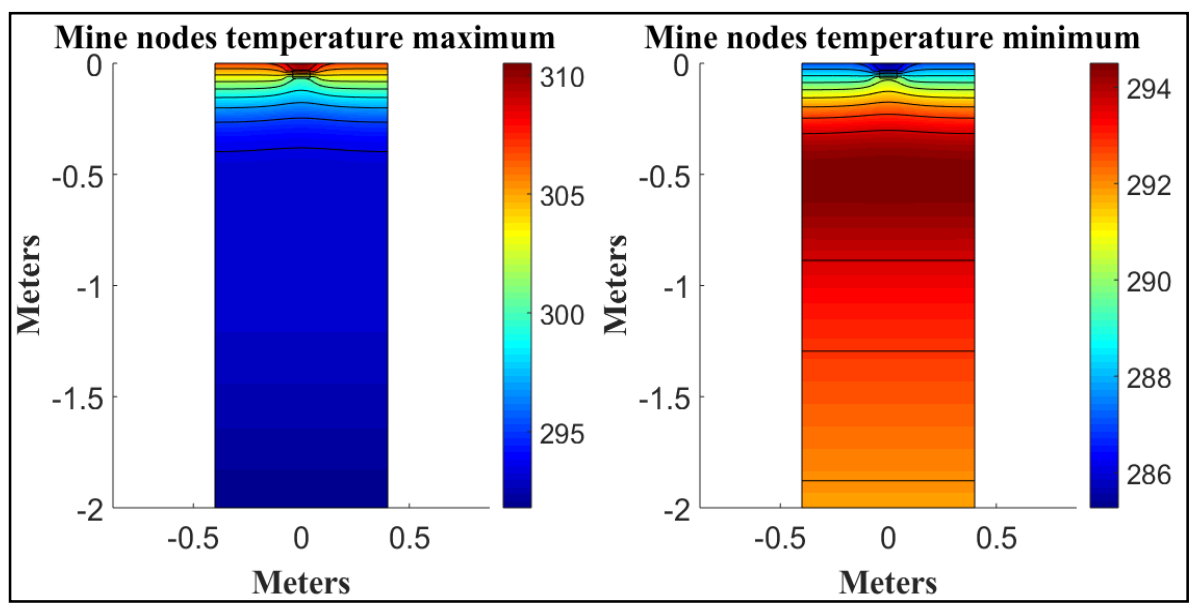

Figure 105 Cross-section of temperature distribution when the PL encountered soil surface, and soil surface's temperature differences are maximum

The PL's influence is inversely proportional to the buried depth of the PL as expected. However, the temperature anomaly that is caused by the metal PL is almost twice larger than plastic PL's when the depth of PL is seven $\mathrm{cm}$. Because of that, detecting deeper metal PLs is easier than plastic counterparts with the IR-based method.

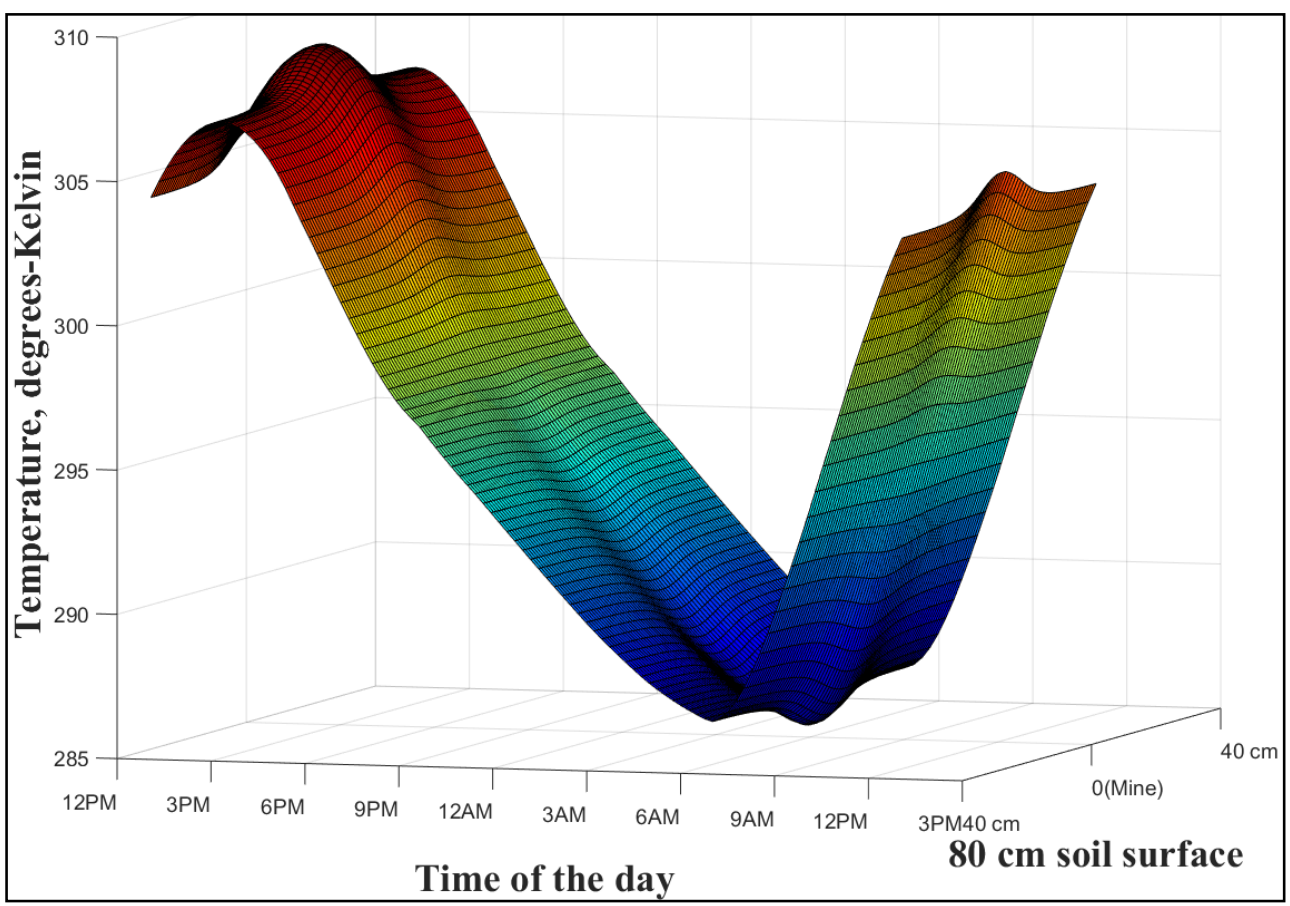

Figure 106 3-D the temperature distribution on the soil surface during diurnal cycle $($ depth $=7 \mathrm{~cm})$ 


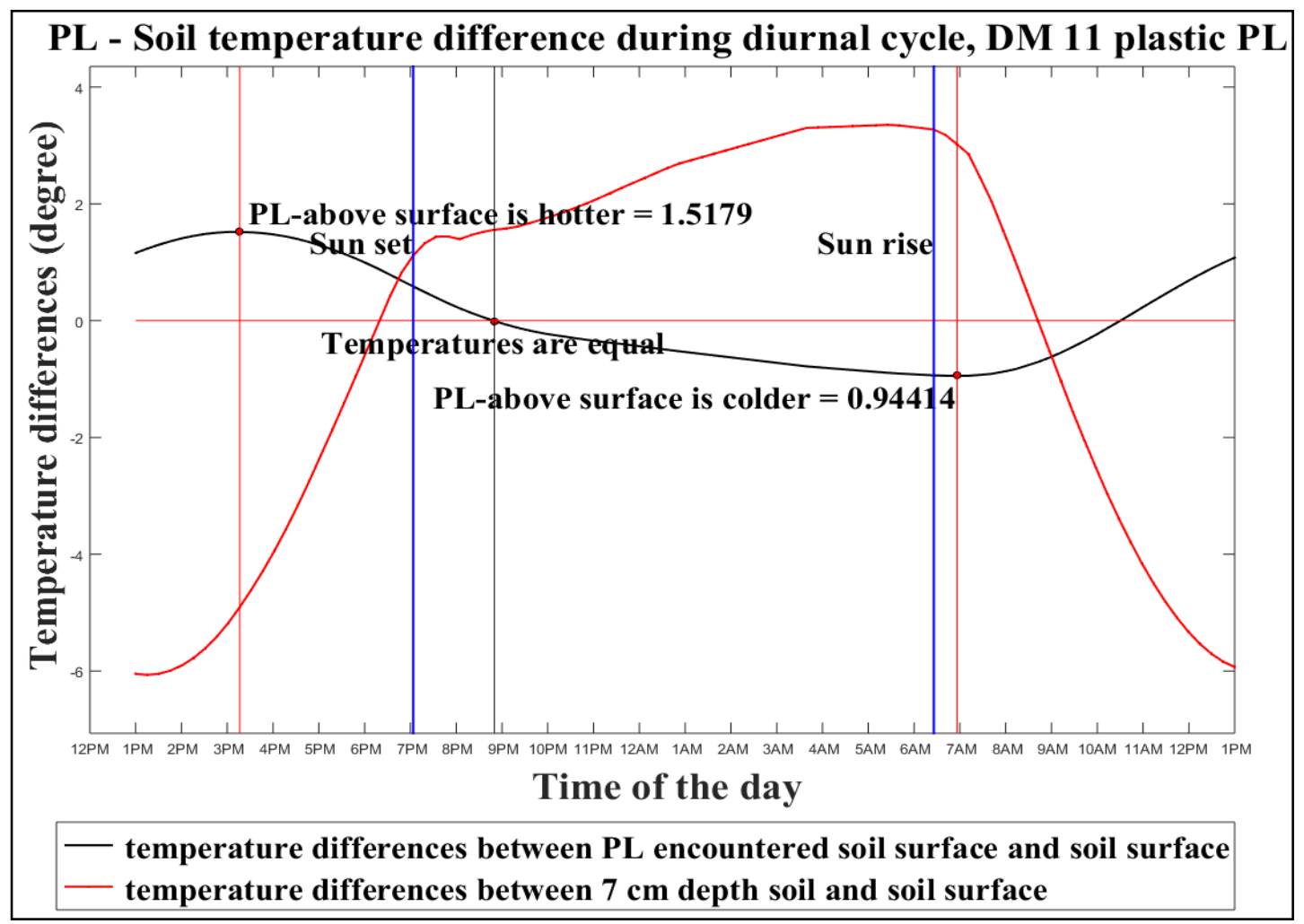

Figure 107 Soil surface temperature and PL encountered soil surface/deep soil temperature difference during diurnal cycle $($ depth $=7 \mathrm{~cm})$

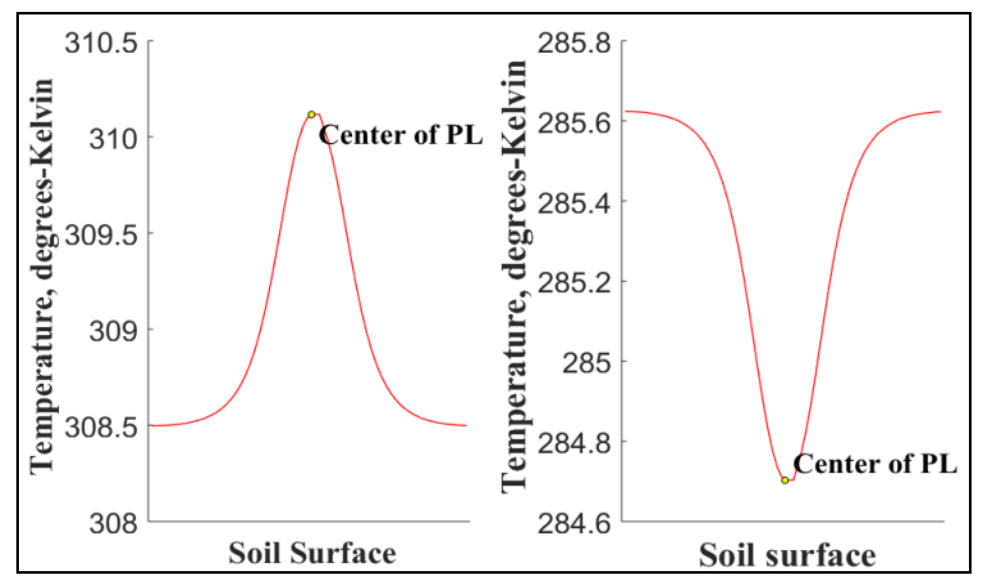

Figure 108 The soil surface temperature distribution $($ depth $=7 \mathrm{~cm})$ 


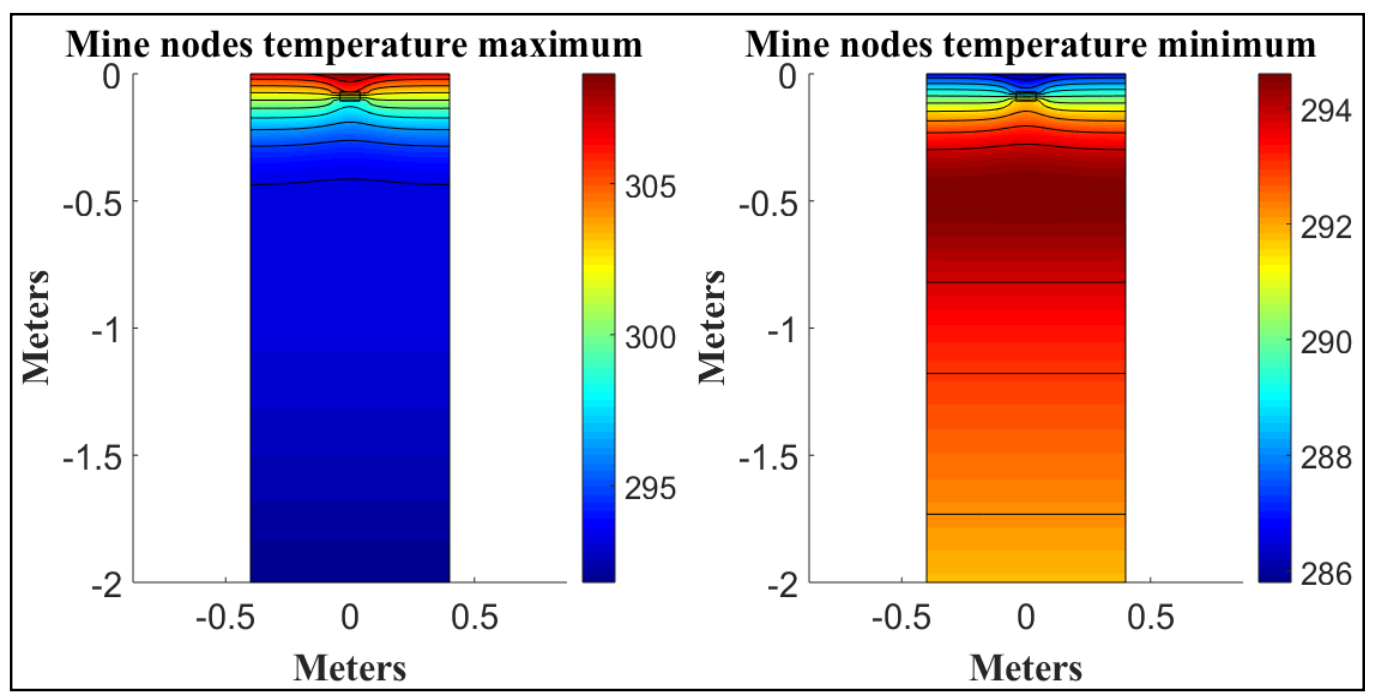

Figure 109 Cross-section of temperature distribution when the PL encountered soil surface, and soil surface's temperature differences are maximum

The entire solution for first test area's simulation is summarized in Table 23 .

Table 23 First test area based simulation solution

\begin{tabular}{|l|c|c|c|c|c|c|}
\cline { 2 - 7 } \multicolumn{1}{c|}{} & \multicolumn{3}{c|}{ M48 Metal PL } & \multicolumn{3}{c|}{ DM11 Plastic PL } \\
\hline Depth of PL(cm) & 1 & 3 & 7 & 1 & 3 & 7 \\
\hline Max. Temperature & 6.35 & 4.98 & 2.82 & 5.33 & 3.29 & 1.52 \\
\cline { 2 - 7 } Difference(degree) & 3.70 & 3.07 & 1.79 & 3.09 & 1.96 & 0.94 \\
\hline Max. Temperature & $2: 00$ & $2: 00$ & $3: 30$ & $1: 30$ & $1: 45$ & $3: 15$ \\
& $\mathrm{pm}$ & $\mathrm{pm}$ & $\mathrm{pm}$ & $\mathrm{pm}$ & $\mathrm{pm}$ & $\mathrm{pm}$ \\
\cline { 2 - 7 } & $5: 15$ & $5: 45$ & $6: 45$ & $5: 15$ & $5: 15$ & $7: 00$ \\
Difference's Hours & $\mathrm{am}$ & $\mathrm{am}$ & $\mathrm{am}$ & $\mathrm{am}$ & $\mathrm{am}$ & $\mathrm{am}$ \\
& \multicolumn{7}{c|}{$7: 10 \mathrm{pm}$} \\
\hline Sunset & \multicolumn{7}{c|}{$6: 23 \mathrm{am}$} \\
\hline Sunrise & \multicolumn{7}{c|}{} \\
\hline
\end{tabular}

It can be inferred that maximum differences occur from 1:30 pm to $3: 15 \mathrm{pm}$.

\subsection{DM11 PL Simulation Results with the Second Test Area's Parameters}

The second test area's soil was moist when the data was captured (8-9 March 2016). Therefore, the moist clay's thermal conductivity is used in this section. Twelve DM11 plastic PLs were buried at three and four DM11 were buried at four cm depth in the second test area. 
3-D simulation result of temperature distribution is given in Figure 109, and PL's caused anomaly in $30 \mathrm{~cm}$ soil surface is given in Figure 111 with different fields of view. The PL is located at the center of $30 \mathrm{~cm}$ cross-section. The soil surface above the PL is colder in both simulation and real data during the nighttime. In addition, the PL encountered soil surface reached the coldest temperature around at 6:00 am, which almost coincides with the sunrise hour, in both solutions. However, in simulation results the maximum positive anomaly occurs at 2:00 pm (Figure 112). Nevertheless, in the real results there are not any significant temperature anomalies.

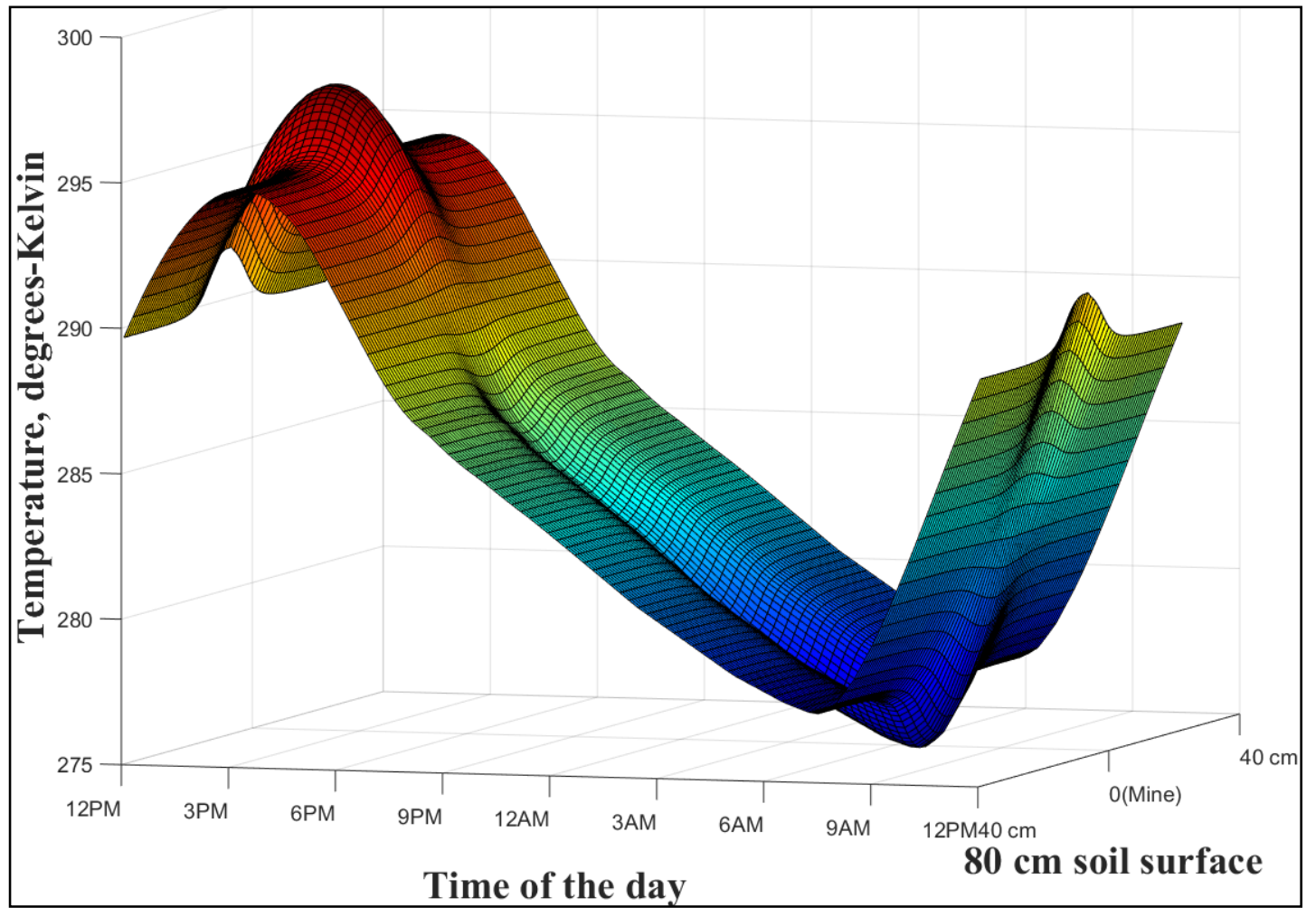

Figure 1103 -D the temperature distribution on the soil surface during diurnal cycle $($ depth $=3 \mathrm{~cm})$

During daytime inconsistent temperature-changes are captured by the IR camera. These inconsistencies occur because of the clutter. The primary clutter sources are the effect of surface reflected sunlight and sky light on not perfectly smooth surface during the daytime. The clutter lessens the performance of IR cameras during daytime [13]. However, simulation's daytime portion gives the largest temperature differences because 
the clutter's influences and signal to noise ratio are not included in the simulation. To sum up, the nighttime images are better for detecting surface anomalies as mentioned and showed in the results in 7.3.7. The temperature distributions on the soil surface and inside the soil are given in Figure 113 and Figure 114 respectively.

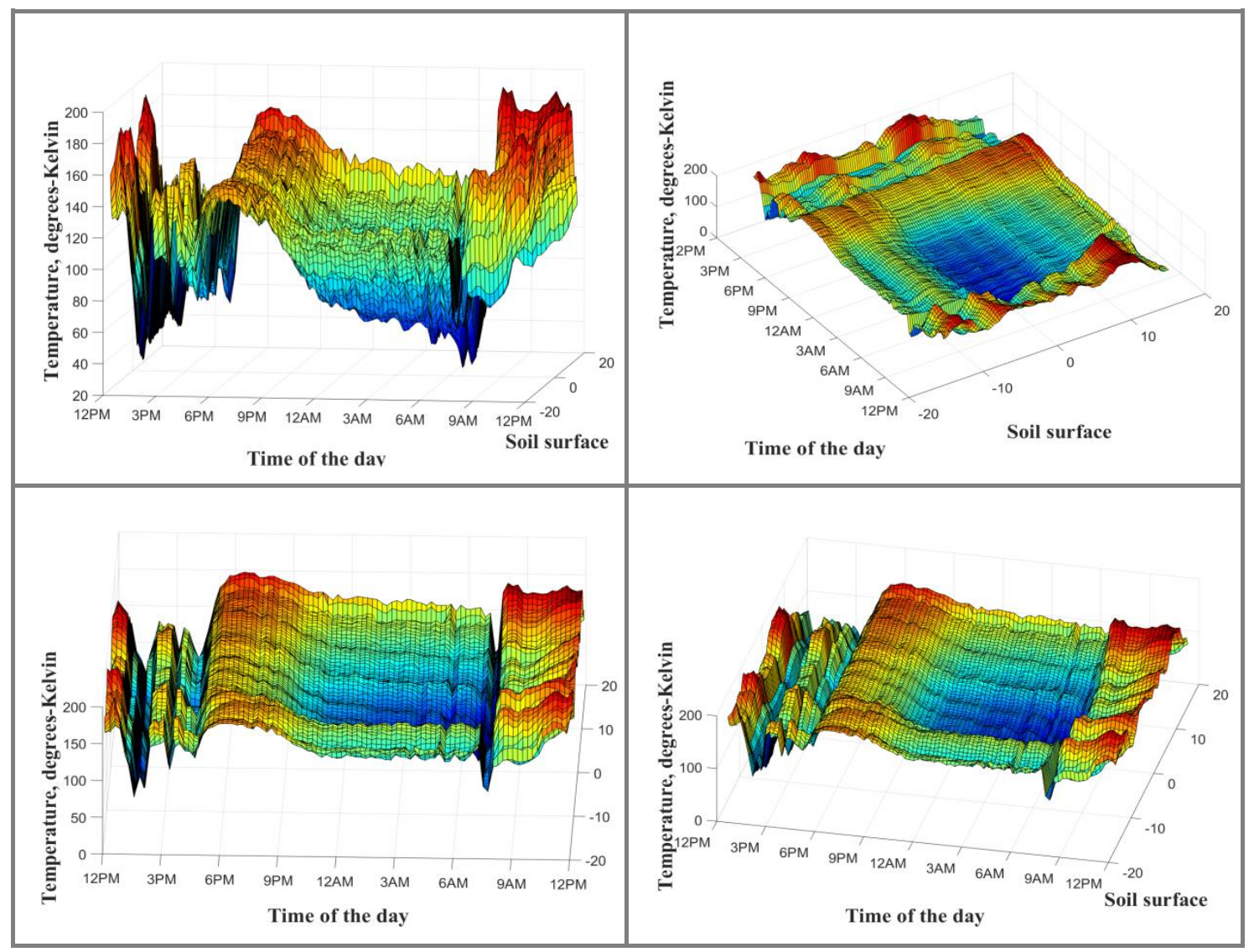

Figure 111 Diurnal temperature distribution of $3 \mathrm{~cm}$ buried PL 


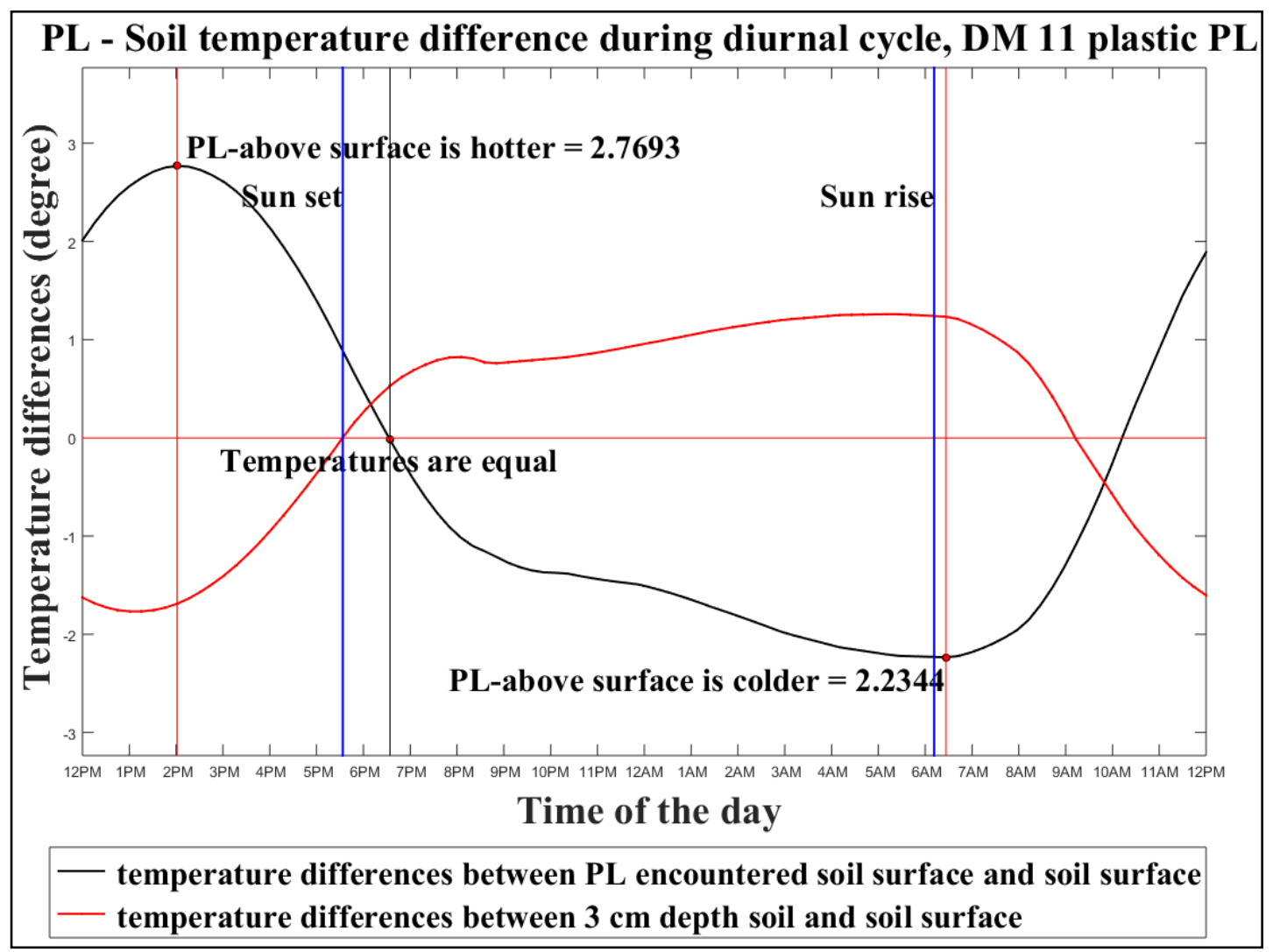

Figure 112 Soil surface temperature and PL encountered soil surface/deep soil temperature difference during diurnal cycle $($ depth $=3 \mathrm{~cm})$

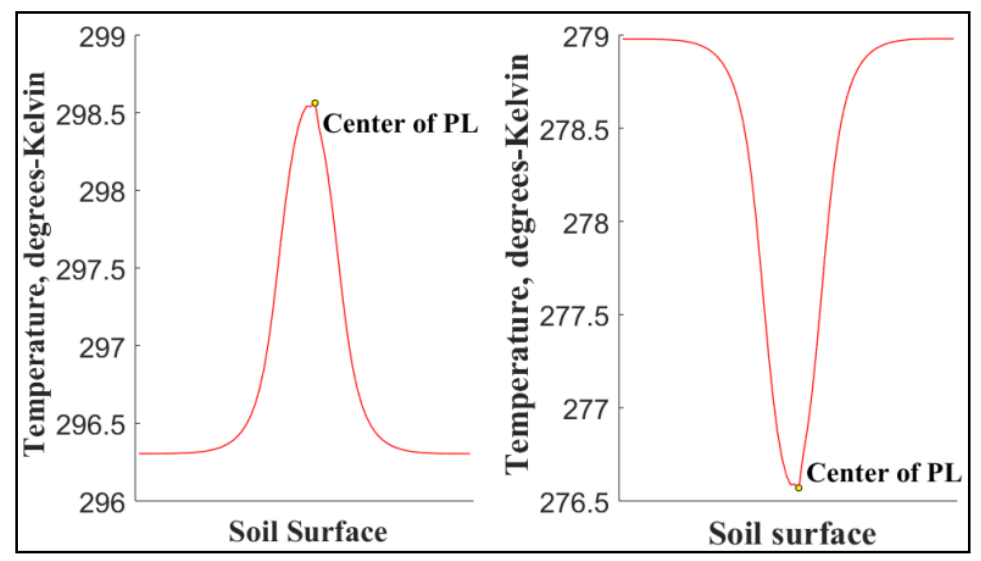

Figure 113 The soil surface temperature distribution $($ depth $=3 \mathrm{~cm})$ 


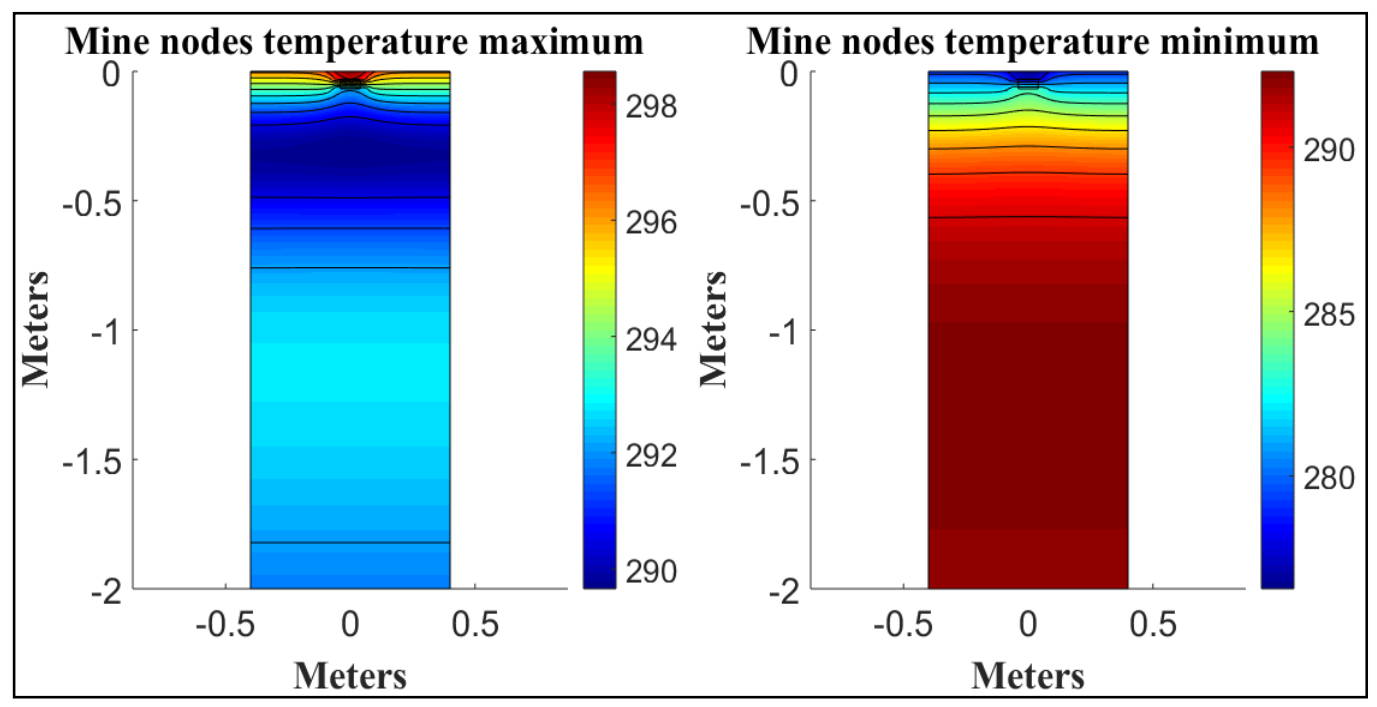

Figure 114 Cross-section of temperature distribution when the PL encountered soil surface, and soil surface's temperature differences are maximum

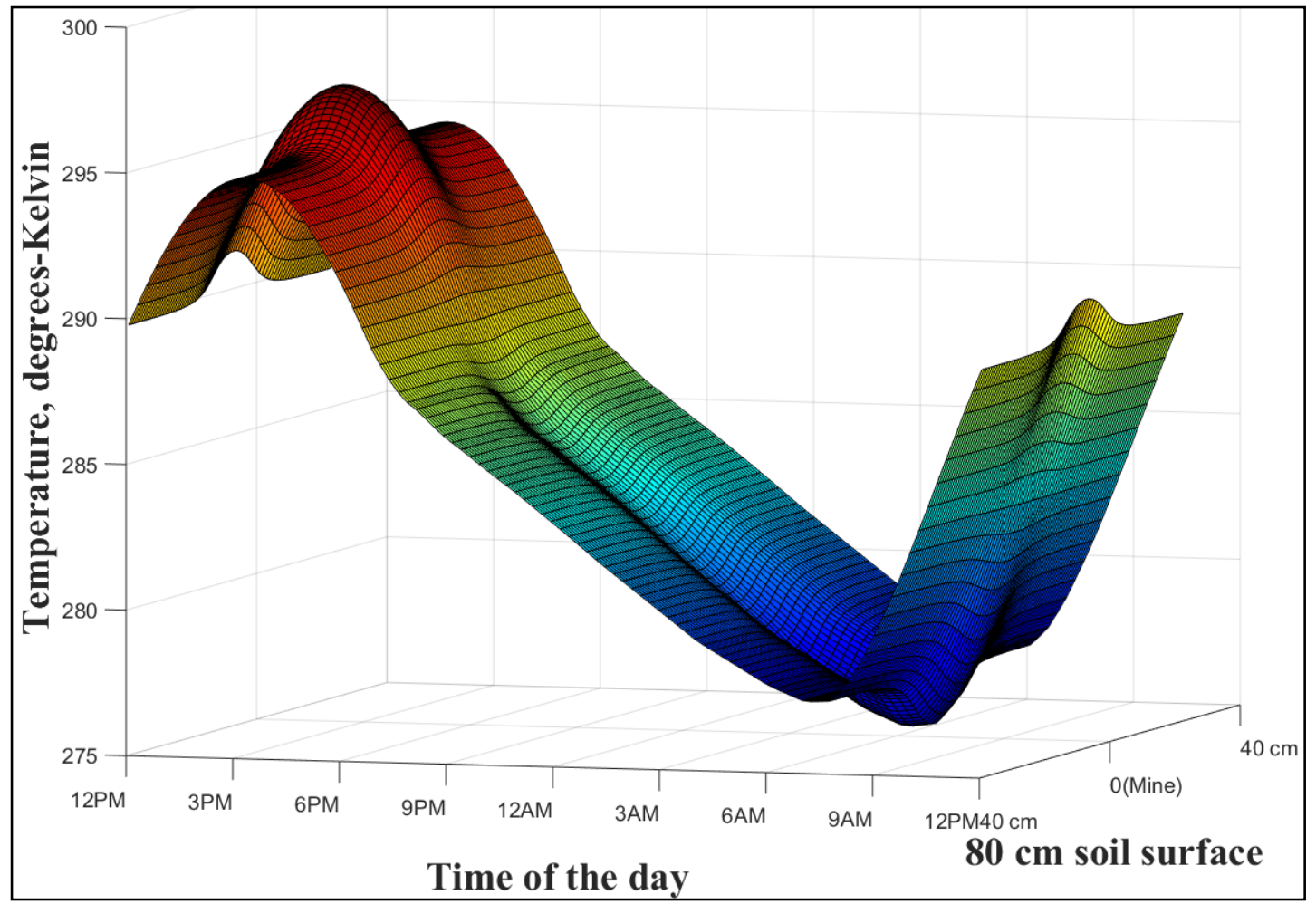

Figure $1153-\mathrm{D}$ the temperature distribution on the soil surface during diurnal cycle $($ depth $=4 \mathrm{~cm})$ 
If the burial depth increases to four $\mathrm{cm}$, the solutions occurred as below. The results are given in Figure 115 - Figure 118. The overall simulation results are given in Table 24.

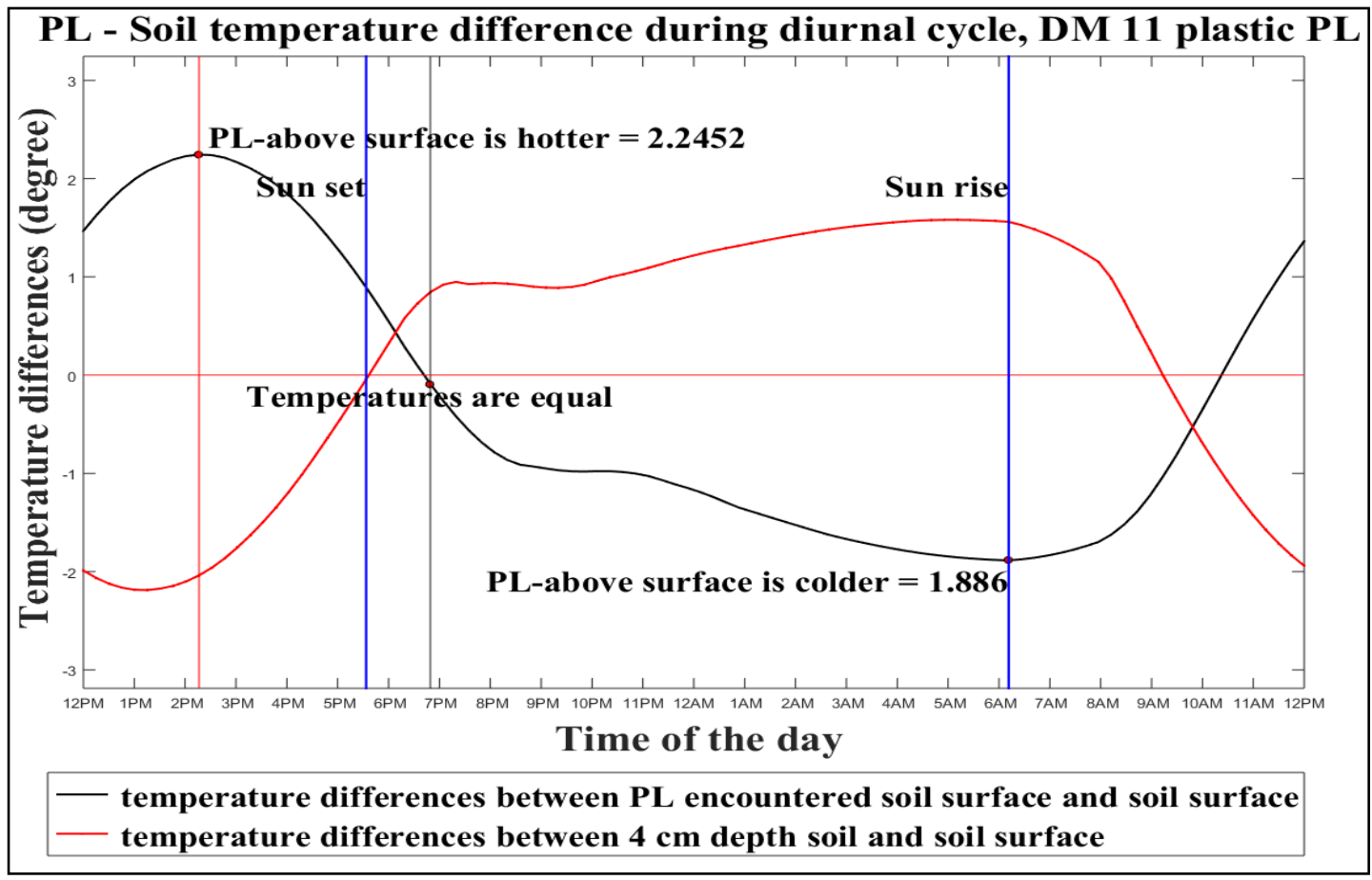

Figure 116 Soil surface temperature and PL encountered soil surface/deep soil temperature difference during diurnal cycle $($ depth $=4 \mathrm{~cm})$

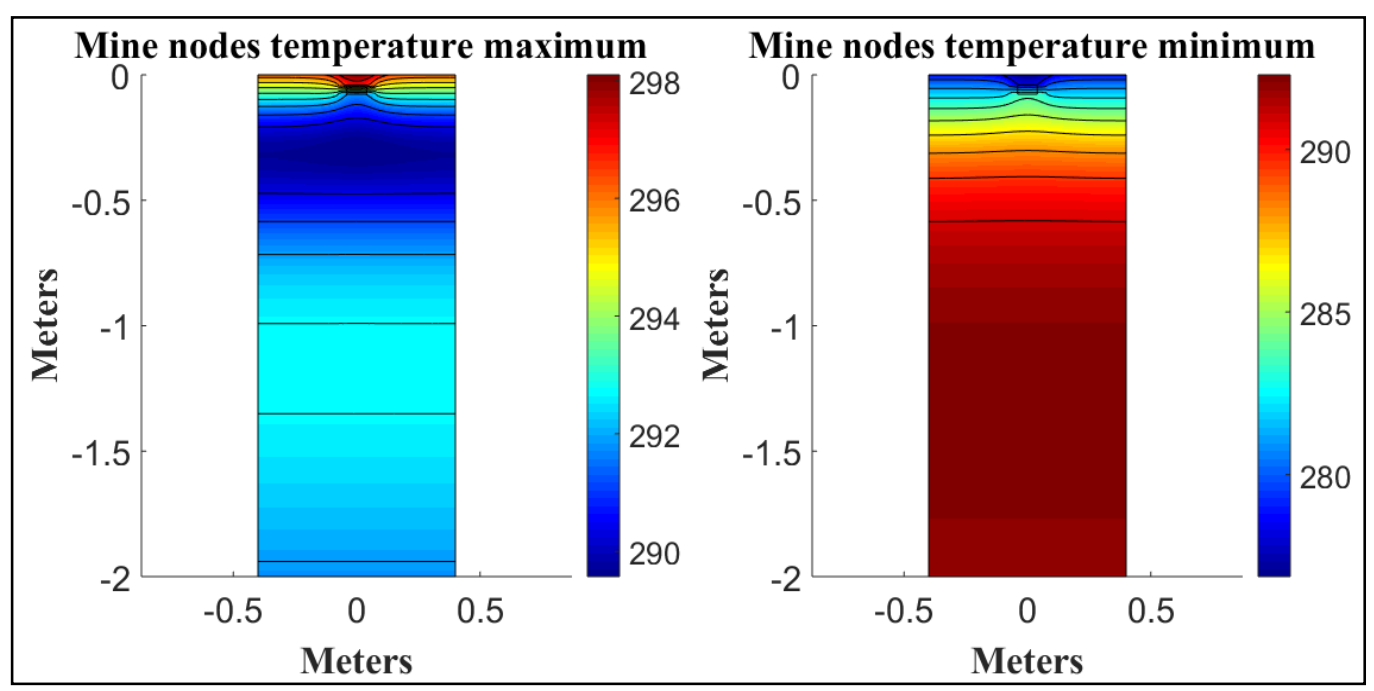

Figure 117 Cross-section of temperature distribution when the PL encountered soil surface, and soil surface's temperature differences are maximum 


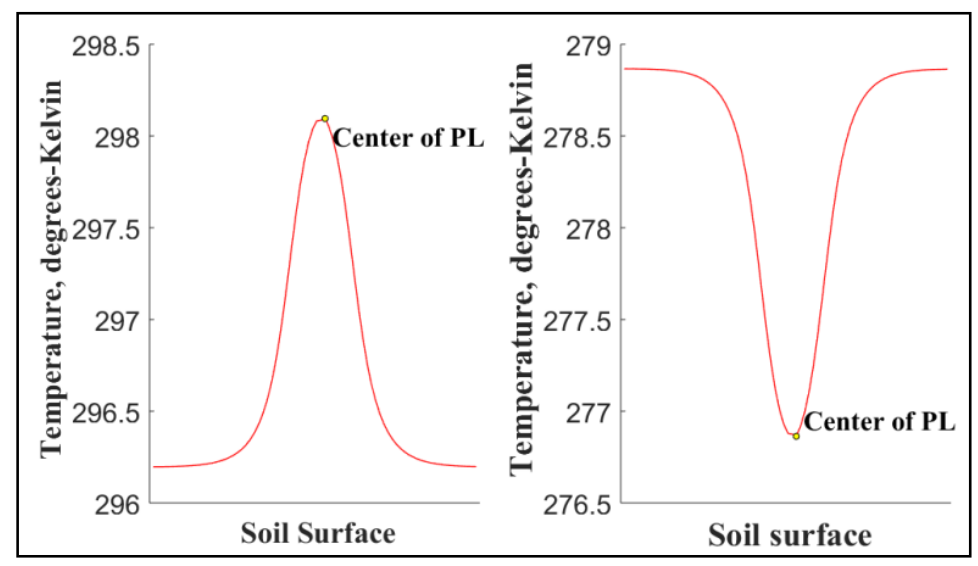

Figure 118 The soil surface temperature distribution $($ depth $=4 \mathrm{~cm})$

Table 24 Simulation solutions for the second test area

\begin{tabular}{|l|c|c|}
\cline { 2 - 3 } \multicolumn{1}{c|}{} & \multicolumn{2}{c|}{ DM11 Plastic PL } \\
\hline Depth of PL (cm) & 3 & 4 \\
\hline \multirow{2}{*}{ Max. Temperature Difference (degree) } & 2.7693 & 2.2452 \\
\cline { 2 - 3 } & 2.2344 & 1.8860 \\
\hline \multirow{2}{*}{ Max. Temperature's Times } & $2: 00 \mathrm{pm}$ & $2: 20 \mathrm{pm}$ \\
\cline { 2 - 3 } & $6: 30 \mathrm{am}$ & $6: 15 \mathrm{am}$ \\
\hline Sunset & \multicolumn{2}{|c|}{$5: 48 \mathrm{pm}$} \\
\hline Sunrise & \multicolumn{2}{|c|}{$6: 09 \mathrm{am}$} \\
\hline
\end{tabular}

When we compare the first test area simulation and the second test area simulation, we can see that at the same depth, the occurring maximum temperature differences are larger in the second test area. This can be seen even by naked eye in Figure 119. Figure 119 (a) and Figure 119 (b) are images captured at 6:00 am from the first and the second test areas respectively. The DM11s' locations are clearer on the second test area's image. The blurry black areas are PLs buried areas in Figure 119 (b).

The visible phenomenon can be explained with the different thermal conductivities. The second minefield's soil thermal conductivity is larger because of moisture content of the soil. Water fills the air gap in the soil and increases the thermal conductivity. The numeric solutions, which we got from the simulation results, also support this interpretation. The maximum difference at three cm depth was occurred $2.24^{\circ}$ in second test area and this value is $1.96^{\circ}$ in the first test area. 


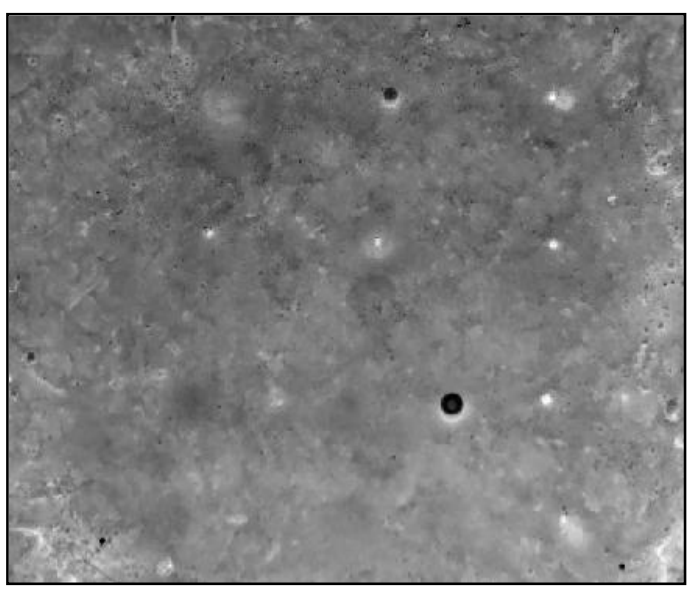

(a)

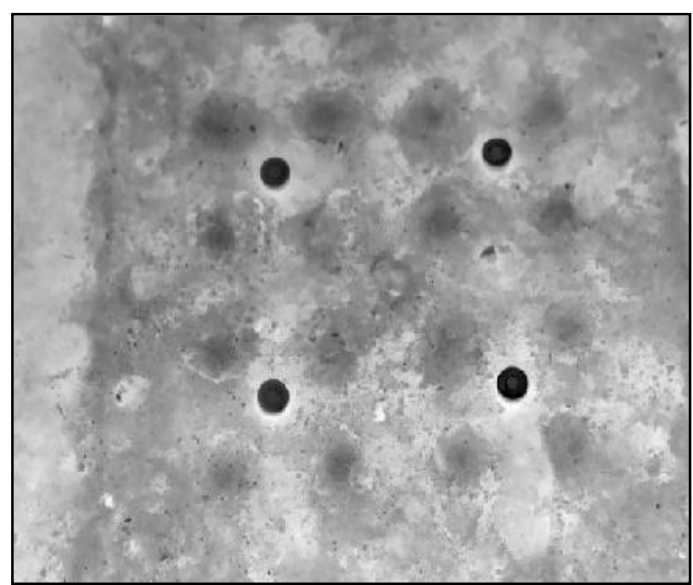

(b)

Figure 119 Images captured at 6:00 am from first and second test areas

Currently most of the mine detection and cleaning operations are done during summer times when the soil is dry; however, if the IR based systems are used, either these operations should be done during spring times or the minefields should be humidified in order to increase soil thermal conductivity.

\subsection{Contributions of Simulation Results to the Study}

The results of the simulations and the results of two real data sets show that maximum temperature differences occur approximately within the time range from two hours before sunrise to two hours after. However, the first maximum temperature difference that occurs in the afternoon cannot be used because of the clutter and hence relatively low signal to noise ratio with regard to nighttime images. In addition, maximum temperature gradient occurs within the time range approximately from one hour before the sunset to two hours after. These inferences can be made when the experimental results (Figure 47, Figure 60, Figure 62) and the simulation results (Figure 89, Figure 93, Figure 96, Figure 99, Figure 103, Figure 107, Figure 112, Figure 116) are compared.

At this point one opportunity appears. This opportunity is to decrease the data capturing, filtering times and to get rid of PCA step from the proposed method, which also lessen 
the detection time. This can be possible if sufficient detection results are obtained with smaller image cube.

Detection results acquired by using filtered image cubes that were captured in the time ranges of 4:45 am - 7:45 am, 4:00 am - 7:15 am and 9:20 pm - 7:05 am and QDA classification method are given in Figure 120, Figure 121 and Figure 122. The detection rates, false alarm rates, and detection algorithm processing times are given in Table 25 .

Even though there is one object-based False Positive alarm at each 4:00 am - 7:15 am, and 5:00 pm - 8:00 pm periods processed detection result, the detection rates, and the false alarm rates are quite good with regard to nine hours 45 min image cube result. There is not any false positive alarm at 4:45 am - 7:45 am image cube result, however, detection rate is not as good as others. Thus, using three to four hours data from the sunset and the sunrise periods give opportunity to detect all PLs with high detection rates and low false alarm rates without applying PCA transformation and finding optimum PCA size. In addition, even though detection duration differences, which are given in Table 25, are not significant, data capturing time decrease from approximately from 10 hours to three hours.

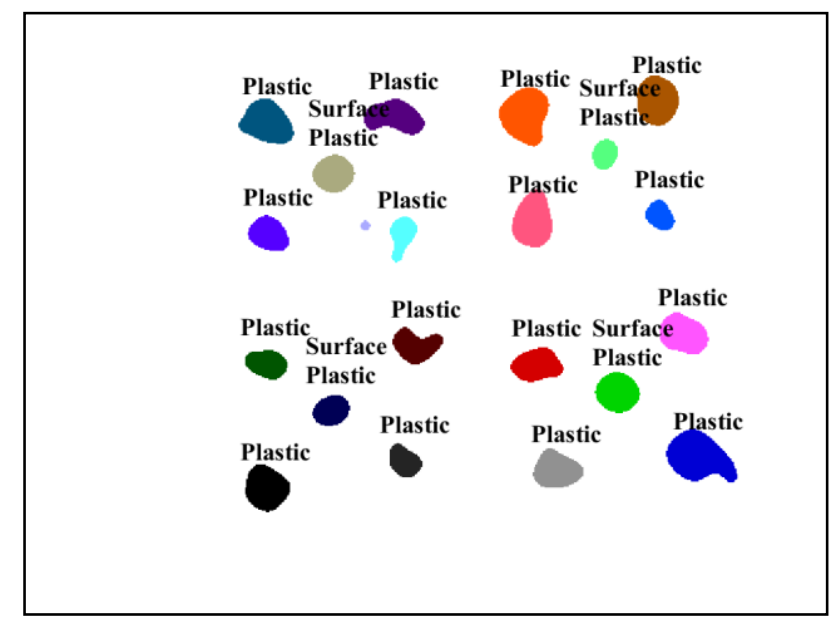

Figure 120 4:45 am - 7:45 am period object-based detection result 


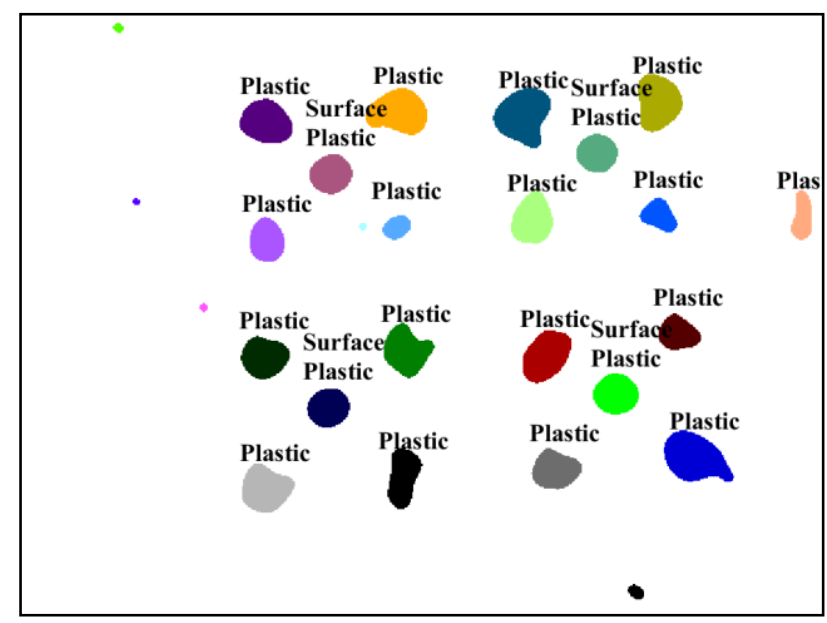

Figure 121 4:00 am - 7:15 am period object-based detection result

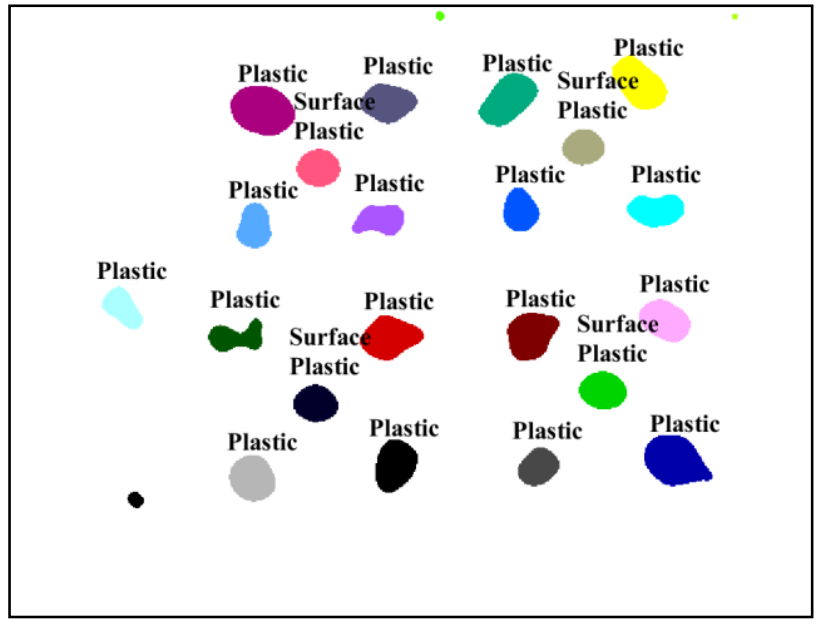

Figure 122 5:00 pm - 8:00 pm period object detection result

Table 25 Detection results with respect to time intervals

\begin{tabular}{|c|c|c|c|}
\hline Imaging Period & False Alarm Rate (\%) & Detection Rate (\%) & Detection Time (s) \\
\hline $\begin{array}{c}\text { 4:45 am - 7:45 am } \\
(3 \mathrm{hrs})\end{array}$ & 0.62 & 77 & 209 \\
\hline $\begin{array}{c}\text { 4:00 am - 7:15 am } \\
(3 \mathrm{hrs} 15 \mathrm{~min})\end{array}$ & 0.88 & $\mathbf{8 9}$ & 197 \\
\hline $\begin{array}{c}\text { 9:00 pm - 06:45 am } \\
(\mathbf{9} \text { hrs 45 } \mathbf{m i n})\end{array}$ & 0.70 & $\mathbf{9 0}$ & 202 \\
\hline $\begin{array}{c}\mathbf{5 : 0 0} \mathbf{~ p m ~ - ~ 8 : 0 0 ~} \mathbf{~ p m} \\
(\mathbf{3} \text { hrs })\end{array}$ & $\mathbf{0 . 7 4}$ & $\mathbf{9 4}$ & $\mathbf{1 9 8}$ \\
\hline
\end{tabular}




\section{CHAPTER 9}

\section{CONCLUSIONS}

Thermal differences between the bare soil and the soil surface above buried mines are quite small. In this study, a circularly symmetric spatial filter is applied to amplify these differences. Applying presented spatial filter increases the detection rates and decreases false alarm rates. Since this signal has a dynamical behavior throughout the day, using time series has the benefit of detecting and classifying mines with a high accuracy. It is observed from two different data sets captured in very different conditions that the time between two hours before the sunset, two hours after the sunrise, and nighttimes are the most useful periods.

The period from two hours before sunset/sunrise to after two hours sunset/sunrise is furthermore valuable. Using this period in the proposed algorithm without applying PCA transform allows the detection of all the PLs. This could decrease the processing time, and it could make the execution easier for operators.

Classification algorithms, which are applied on processed data, can detect and classify most of the buried objects with quite small training set. QDA has given the best results with both data sets. This is expected by us because QDA uses individual class means and class covariance different from other maximum likelihood classifiers like MDA or matched filter classifiers. Matched filter classifier uses covariance matrix calculated from the entire data set [45].

Meanwhile the proposed algorithm did not miss (FN) any PL or give any FP alarm when using QDA algorithm in the proposed method when the 10 hours nighttime image cube used. When the three hours sunset and sunrise times used, one FP alarm emerged. 
However, during mine detection and cleaning FP alarms do not cause problem if their numbers are in acceptable range. The biggest threat is undetected mines, and proposed method did not miss any PL even with three hours sunset and sunrise filtered image cubes. In addition, algorithm did not confuse buried stones' pixels with landmine pixels and soil pixels as well.

Temperature anomalies are larger on the soil surface when the soil is moist. This is verified with simulation results using slightly moist second test area's parameters. Thus, mine detection dates should be selected when the searching areas are moist if the IRbased detection system is used. If the time could not be selected according to soil moisture level, humidifying the search area would increase the detection rate.

It is observed that enlarging training set decreases false alarm rates for all algorithms; in addition to that, it increases detection rates for SVM and k-NN. Enlarging only soil pixels size in training set contributes to increasing the detection rate, and decreasing the false alarm rate.

The spatial filter's radii and number of sample points also affects the results. The best results are obtained with 50 pixels $R_{o}$ (outer radii of spatial filter) that corresponds to two times the DM11 diameter and 24-sample points.

The major problem of conventional magnetic field based detection systems is inability to detect buried plastic landmines that has small amount of metal or no metal at all. The proposed algorithms overcome this difficulty of finding plastic and metal landmines thanks to the spatially filtered data.

To further the research, real effects (rough terrain, vegetation, rain, snow etc.) can be investigated. In addition, aircrafts like drones can be used as platforms that carry thermal cameras for capturing images from large test/real minefields and can be worked on with these image series as well. 


\section{REFERENCES}

[1] I. Bottiglero, 120 Million Landmines Deployed Worldwide: Fact or Fiction?, London: Pen \& Sword Books, Ltd., 2000.

[2] Brooks, J., Kempen,L. V., Sahl, H.i, "A Primary Study in Adaptive Clutter Reduction," in In the Proceedings of the SPIE, Detectionand Remediation Technologies for Mines and Minelike Targets V, vol. 4038, pp. 1409-1418, 2000.

[3] R. Bello, "Literature Review on Landmines and Detection Methods," Frontiers in Science, vol. 3, no. 1, pp. 27-42, 2013.

[4] Nath, B. K. and Bhuiyan, A., "A Geometrical Feature Based Sensor Fusion Model of GPR and IR for Detection and Classification of Anti-Personnel Mines," in In ISDA '07:Proceedings of the Seventh International Conference on Intelligent Systems Design and Applications, pages 849-856. IEEE Computer Society, Washington, DC, USA, 2007.

[5] "International Campaign to Ban Landmines. Major Findings," 2014.

[6] Ajlouni, A., Sheta, A.F., "A Novel Landmine Detection Process Using KL Transform and Marker based Watershed Segmentation in IR Images," International Journal of Signal and Imaging Systems Engineering, 2010.

[7] Kasban, H., Zahran, O., Elaraby, S., M., El-Kordy, M., "A Comparative Study of Landmine DetectionTechniques," Sens Imaging (2010) 11:89-112, p. 89-112, 2010.

[8] Gonzalez, P., Cobano, J. A., Garcia, E., Estremera, J., \& Armada, M. A., "A sixlegged robot-based system for humanitarian demining missions," Mechatronics, vol. 17(8), pp. 417-430, 2007.

[9] Khanafer K., Vafai K., and Baertlein, B. A., "Effects of Thin Metal Outer Case and Top Air Gap on Thermal IR Images of Buried Antitank and Antipersonnel Land Mines," IEEE Transactions on Geoscience and Remote Sensing, vol. 41, no. 1, pp. 123-135, 2003. 
[10] Nguyen, T., T., Sahli, H. and Nho, H., D., "Thermal infrared technique for landmine detection: Mathematical formulation and methods," RICAM, 2011.

[11] Lillesand, T., M., Kiefer, R., W., Chipman, J., W., Remote Sensing and Image Interpretation, John Wiley \& Sons,Inc., 2007.

[12] Richards, J., A. and Jia, X., "The Effect of the Atmosphere on Radiation," in Remote Sensing Digital Image Analysis An Introduction, Canberra, Springer, 2005, p. 28.

[13] Sendur, Ibrahim K. and Baertlein, Brian A., "Techniques for improving buried mine detection in thermal IR imagery," in 3710, Detection and Remediation Technologies for Mines and Minelike Targets IV, 1999.

[14] Rao, K., R. and Yip, P., C., The Transform and Data Compression Handbook, Boca Raton: CRS Press LLC, 2001.

[15] Duda, R., O., Hart, P., E., Stork, D., G., "10.13 Comp onent analysis," in Pattern Classification, John Wiley \& Sons, 2001, p. 53.

[16] Sen, S., \& Woodfin, R. L., "Humanitarian De-mining and the Quest for Better Ways of Locating Buried Non-Metallic Objects," Physics and Society (Newsletter of the American Physical Society Forum), pp. 7-9, April 2002.

[17] Zhang, J., Liu, Q., and Nath,B., "Image segmentation based on competitive learning," Journal of marine science and Application, vol. 3, pp. 71-74, 2004.

[18] C. P. Lee, "Landmine detection techniques using multiple sensors, M. Sc. Thesis, Electrical," 2004.

[19] Masunaga, S., \& Nonami, K., "Controlled metal detector mounted on landmine detection," International Journal of Advanced Robotic Systems, no. 4(2), p. 237245, 2007.

[20] Paik, J., Lee, C., P. \& Abidi, M., A.i, "Image Processing-Based Mine Detection Techniques:A Review," Subsurface Sensing Technologies and Applications, vol. 3, no. 3, 2002.

[21] Bruschini, C. \& Gros, B., "A survey of Current Sensor Technology Research for the 
detection of landmines," in In the proceedings of International workshop on Sustainable Humanitarian Demining, 1997.

[22] C. Santulli, "CARLO SANTULli HOME PAGE," 2007. [Online]. Available: http://www.carlosantulli.net/currypub.html. [Accessed 11 March 2016].

[23] Cremer, F., Nguyen, T. T. , Yang, L. \& Sahli, H., "Stand-off Thermal IR Minefield Survey: System concept and experimental results," in Proceedings of the SPIE, Vol. 5794, pp 209 - 220., 2005.

[24] Dam, R. L. V., Borchers, B., Hendrickx, J. M. H. \& Harmon, R. S., "Effects of soil water content and texture on radar and infrared landmine sensors:implications for sensor fusion," in In the proceedings of European Demining, 2003, 2003.

[25] Bruschini, C., \& Gros, B., "A Survey of research on sensor technology for landmine detection," Journal of Humanitarian Demining, no. 2.1, 1998.

[26] Khanafer, K., Vafai, K., "Thermal analysis of buried land mines over a diurnal cycle," Geoscience and Remote Sensing, vol. 40, no. 2, pp. 461-473, 2002.

[27] Hong, S. H., Miller, T. W., Borchers, B., Hendrickx, J. M., Lensen, H. A., Schwering, P. B., \& Van Den Broek, S. P., " Land mine detection in bare soils using thermal infrared sensors," in In AeroSense 2002 (pp. 43-50). International Society for Optics and Photonics., 2002.

[28] Ajlouni, A. and Sheta, A., "Landmind detection with IR sensors using karhunen loeve transformation and watershed segmentation.," in The 5th IEEE International Multi-Conference on Systems, Signals and Devices,pages 1-6. , 2008.

[29] Nguyen, T., Hao, D., P. Lopez, F. C., and Sahli, H., "Thermal infrared identification of buried landmines," in In Proceedings of the SPIE, volume 45794, pages 198-206., 2005.

[30] Martínez, P.,L., Kempen, L.,v., Sahli, H., Ferrer, D., C., "Improved Thermal Analysis of Buried Landmines," Transactions on Geoscience and Remote Sensing, vol. 42, no. 9, 2004.

[31] Sendur, Ibrahim K. and Baertlein, Brian A., "Numerical simulation of thermal 
signatures of buried mines over a diurnal cycle," in SPIE 4038, Detection and Remediation Technologies for Mines and Minelike Targets V, 2000.

[32] Thành, N., T., Sahli, H., and Hào, D., N. , "Finite-Difference Methods and Validity of a Thermal Model for Landmine Detection With Soil Property Estimation," IEEE Transactions on Geoscience and Remote Sensing, vol. 45, no. 3, pp. 656-674, 2007.

[33] Thành,N., T., Sahli, H., and Hào, D., N., "Infrared Thermography for Buried Landmine Detection: Inverse Problem Setting," Transactions on Geoscience and Remote Sensing, vol. 46, no. 12, 2008.

[34] Muscio, A., Corticelli, M., A., "Experiments of thermographic landmine detection with reduced size and compressed time," Infrared Physics \& Technology, vol. 46, no. 1-2, pp. 101-107, 2004.

[35] Bhuiyan, A. and Nath, B., "Anti-personnel landmine detection based on GPR and IR imaging: A review," Computer Science and Software, Engineering, The University of Melbourne, Melbourne, 2006a.

[36] D. o. t. U. A. Headquarters, "SECTION II. ANTITANK MINES," in Mine/Countermine, Washington, DC, Headquarters, Department of the Army, 2004, pp. A-11.

[37] Moukalled, F., Ghaddar, N., Saleh, Y., Fawaz, Z., "Heat and mass transfer in moist soil, part II. Application to predicting thermal signatures of buried landmines," Numerical Heat Transfer Part B-Fundamentals, vol. 49, no. 5, pp. 487-512, 2006.

[38] Sjokvist, S., Linderhed, A., Nyberg, S., Uppsall, M., S., "Temporal method for IR minefield feature detection," in SPIE 5415, 2004.

[39] D. Hillel, Introduction to Soil Physics, New York: Academic Press, 1982, p. 162.

[40] Carslaw, H., S. and Jaeger,J., C., Conduction of Heat in Solids, 2nd ed., Oxford, U.K.: Oxford Univ. Press, 1959.

[41] "Climate Education for K-12," NC STATE UNIVERSITY, 1 August 2013. [Online]. Available: https://climate.ncsu.edu/edu/k12/.LWSW. [Accessed 18 April 2016]. 
[42] A. W. Allen, Astrophysical Quantities, London, London: Athlone, 1963, p. 127.

[43] A. B. Kahle, "“A simple thermal model of the earth's surface for geologic mapping by remote sensing," Journal of Geophysical Research, vol. 82, pp. 1673-1680, 1977.

[44] Lundberg, M., Gu, I., Y., H., "3D matched filter for detection of land mines using spatio-temporal thermal modeling," in SPIE 4038, Detection and Remediation Technologies for Mines and Minelike Targets V, 179, 2000.

[45] D. A. Landgrebe, Signal Theory Methods in Multispectral Remote Sensing, New Jersey: John Wiley \& Sons, 2003. 